\title{
Measurement of $\mathrm{t} \overline{\mathrm{t}}$ cross section in $\mathrm{p} \overline{\mathrm{p}}$ Collider at $\sqrt{\mathrm{s}}=1.96 \mathrm{TeV}$
}

\author{
A Thesis \\ Submitted to the \\ Tata Institute of Fundamental Research, Mumbai \\ for the degree of Doctor of Philosophy \\ in Physics \\ by \\ Prolay Kumar Mal
}

April, 2005 

To the memory of my beloved parents 



\section{DECLARATION}

This dissertation is a presentation of my original work. Wherever contributions of others are involved, every effort is made to indicate this clearly, with due reference to the literature, and acknowledegment of collaborative research and discussions.

This work was done under the guidance of Professor Sudeshna Banerjee, at the Tata Institute of Fundamental Research, Mumbai.

In my capacity as supervisor of the candidate's thesis, I certify that the above statements are true to the best of my knowledge.

Date:

Signature of the Supervisor

(Prof. Sudeshna Banerjee) 



\section{Contents}

$\begin{array}{ll}\text { Synopsis } & \text { ix }\end{array}$

List of Publications $\quad$ xxiii

Acknowledgement $\quad$ Xxv

List of Figures $\quad$ xxxiii

List of Tables $\quad$ xxxvii

1 Introduction 1

1.1 History . . . . . . . . . . . . . . . . 2

1.2 The Standard Model . . . . . . . . . . . . . . . . . 7

1.3 Top Quark. . . . . . . . . . . . . . . 15

1.3.1 Important Aspects of Top Quark . . . . . . . . . . . 16

1.3.2 Production of Top Quark at Tevatron . . . . . . . . . . 20

1.3.3 Decay of Top Quark . . . . . . . . . . . . . 28

1.3.4 Experimental Signature . . . . . . . . . . 30

1.4 The $e \mu$ channel . . . . . . . . . . . . . . . . . . . 32

1.4.1 Event Signature . . . . . . . . . . . . . . 33 
1.4.2 Background .................. 34

2 Experimental Apparatus $\quad 41$

2.1 Tevatron Accelerator System . . . . . . . . . . . . . . . . . . . 42

2.1.1 Proton Production . . . . . . . . . . . . . . 42

2.1.2 Antiproton Production . . . . . . . . . . . . . . 47

2.1.3 The Tevatron Ring . . . . . . . . . . . . 50

2.2 DØ Detector . . . . . . . . . . . . . . . . 52

2.2.1 Coordinate System . . . . . . . . . . 53

2.2.2 Luminosity Monitors . . . . . . . . . . . . 57

2.2.3 Central Tracking System . . . . . . . . . . . 59

2.2.4 Central Preshower Detector . . . . . . . . . . . . . 69

2.2.5 Forward Preshower Detectors ......... . 71

2.2.6 Calorimeter .................. . . 73

2.2.7 Muon System . . . . . . . . . . . . . 85

2.2.8 Trigger and Data Acquisition System . . . . . . . . 93

3 Event Reconstruction 101

3.1 The D $\varnothing$ Reconstruction Program . . . . . . . . . . . . 102

3.1.1 Track Reconstruction . . . . . . . . . . . . . . . . 104

3.1.2 Event Vertex Determination . . . . . . . . . . 110

3.1.3 Calorimeter Reconstruction ... . . . . . . . . . 113

3.2 Particle Identification . . . . . . . . . . . . . . . . 115

3.2.1 Electrons . . . . . . . . . . . . . . . . . 115

3.2.2 Electromagnetic Energy Correction . . . . . . . . . 128

3.2 .3 Muons ..................... . 134

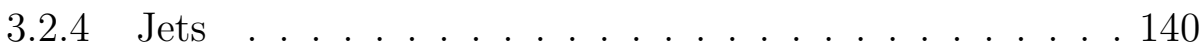


3.2.5 Jet Energy Calibration . . . . . . . . . . . . . 147

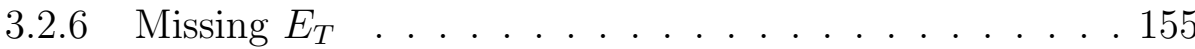

4 Data and Monte Carlo 157

4.1 Data Sample. . . . . . . . . . . . . . . . . . 157

4.1.1 Integrated Luminosity . . . . . . . . . . . . . . . . . . 158

4.1.2 Data Quality ................. 161

4.2 Trigger . . . . . . . . . . . . . . . . . . . 164

4.2.1 Single Object Trigger Efficiency . . . . . . . . . 167

4.2.2 Total Trigger Efficiency . . . . . . . . . . . . . . . . 172

4.3 Monte Carlo . . . . . . . . . . . . . . . . . 176

4.3.1 Event Production . . . . . . . . . . . . . 177

4.3 .2 Event Simulation . . . . . . . . . . . . . . . . 184

4.3.3 Monte Carlo Smearing . . . . . . . . . . . . . . . 185

5 Analysis 199

5.1 Event Selection . . . . . . . . . . . . . . 200

5.2 Optimization of Cuts . . . . . . . . . . . . . 205

5.2.1 Figure of Merit . . . . . . . . . . . . . . . 205

5.2 .2 Grid Search . . . . . . . . . . . . . 206

5.3 Data to MC Scale factors . . . . . . . . . . . . . . . 209

5.3.1 PV Scale Factors . . . . . . . . . . . . . . . . . 210

5.3.2 Scale Factors for Electron selection criteria . . . . . . . 213

5.3.3 Muon Scale Factors . . . . . . . . . . . . . . . . . . . . 219

5.3 .4 Jet Scale Factor . . . . . . . . . . . . . . . . . . 226

5.4 Signal Efficiency . . . . . . . . . . . . . . . . . . . . . 228

5.5 Background Estimation . . . . . . . . . . . . . 230 
5.5.1 Physics Backgrounds ............... 230

5.5.2 Photon Backgrounds . . . . . . . . . . . . . . 231

5.5.3 Instrumental Fakes . . . . . . . . . . . . . . 236

5.6 Final Event Yields . . . . . . . . . . . . . . . . 250

5.7 Systematics . . . . . . . . . . . . . . . . . 262

5.8 Cross section calculation . . . . . . . . . . . . . . 273

$\begin{array}{lr}\text { Conclusion } & 279\end{array}$

A Topological Variables $\quad 283$

A.1 Aplanarity $(\mathcal{A})$ and $\operatorname{Sphericity}(\mathcal{S}) \ldots . . \ldots 283$

A.2 $\tau \tau$ Invariant mass . . . . . . . . . . . . . . . . . . . 285

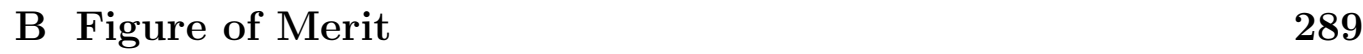

$\begin{array}{ll}\text { C Candidate Events } & 293\end{array}$

C.1 Event Kinematics . . . . . . . . . . . . . . . . 293

C.2 Event Display . . . . . . . . . . . . . . . . . 297

$\begin{array}{ll}\text { Bibliography } & 307\end{array}$ 


\section{Synopsis}

\section{Introduction}

Discovery of the top quark in 1995 at the Fermilab Tevatron collider concluded a long search following the 1977 discovery of bottom (b) quark [1] and represents another triumph of the Standard Model (SM) of elementary particles. Top quark is one of the fundamental fermions in the Standard Model of electroweak interactions and is the weak-isospin partner of the bottom quark. A precise measurement of top pair production cross-section would be a test of Quantum Chromodynamics (QCD) prediction.

Presently, Tevatron is the world's highest energy collider where protons $(p)$ and anti-protons $(\bar{p})$ collide at a centre of mass energy $(\sqrt{s})$ of $1.96 \mathrm{TeV}$. At Tevatron top $(t)$ and anti-top $(\bar{t})$ quarks are predominantly pair produced through strong interactions - quark annihilation $(\simeq 85 \%)$ and gluon fusion $(\simeq 15 \%)$. Due to the large mass of top quark, $t$ or $\bar{t}$ decays $\left(\sim 10^{-25} \mathrm{sec}\right)$ before hadronization and in SM framework, it decays to a $W$ boson and a $b$ quark with $\sim 100 \%$ branching ratio $(\mathrm{BR})$. The subsequent decay of $W$ boson determines the major signatures of $t \bar{t}$ decay. If both $W$ bosons (coming from $t$ and $\bar{t}$ decays) decay into leptons (viz., $e \nu_{e}, \mu \nu_{\mu}$ or $\tau \nu_{\tau}$ ) the corresponding $t \bar{t}$ decay is called dileptonic decay. Of all dileptonic decay 


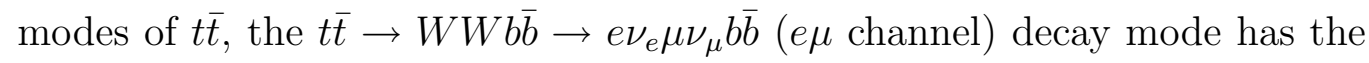
smallest background contamination from $Z^{0}$ production or Drell-Yan process; simultaneously, it has the highest BR ( 3.16\%) [2] amongst all dileptonic decay modes of $t \bar{t}$.

During Run I (1992-1996) of Tevatron, three $e \mu$ candidate events were detected by $\mathrm{D} \varnothing$ experiment, out of 80 candidate events (inclusive of all decay modes of $t \bar{t}$. Due to the rarity of the $t \bar{t}$ events, the measured cross-section has large uncertainty in its value (viz., $5.69 \pm 1.21$ (stat) \pm 1.04 (sys) pb @ $\sqrt{s}=1.8 \mathrm{TeV}$ measured by $\mathrm{D} \varnothing[3])$. This analysis presents a cross section measurement in $e \mu$ channel utilizing $\sim 228 \mathrm{pb}^{-1}$ of data collected by $\mathrm{D} \varnothing$ experiment during Tevatron Run II (between June 2002 and April 2004).

\section{Tevatron \& DØ}

The Tevatron is a superconducting synchrotron that stretches over four miles in circumference. This enormous machine is fed with $p$ and $\bar{p}$ from a complex chain of accelerators (viz., Cockcroft-Walton, Linac, Booster, etc.) and $p$ (or $\bar{p}$ ) gets energized up to $980 \mathrm{GeV}$ resulting in $\sqrt{s}=1.96 \mathrm{TeV}$ (slightly higher than Run I centre of mass energy i.e. $\sqrt{s}=1.8 \mathrm{TeV}$ ). Inside the Tevatron ring, $p \bar{p}$ collisions occur at two points, where the detectors (viz., $\mathrm{D} \varnothing$ and $\mathrm{CDF}$ ) are situated. A typical Run II luminosity i.e., the number of interactions per second per square centimetre is of $O\left(10^{32}\right)$ which is one order of magnitude higher than the Run I luminosity. Integral of this luminosity over time is typically expressed in $p b^{-1}\left(=10^{-36} \mathrm{~cm}^{-2}\right)$ and is a measure of the number of $p \bar{p}$ collisions per unit area during a certain period of time.

The $\mathrm{D} \varnothing$ detector is an assembly of several individual detectors designed to study $p \bar{p}$ collisions at high energies. Many of its components have been up- 
graded after Run I for better performance. The D $\varnothing$ detector contains three major subsystems - a magnetic central tracking system, a hermetic Uranium liquid Argon calorimeter and a muon spectrometer. The Central tracking system is composed of a high resolution silicon detector and a scintillation fibre tracker. For better momenta measurement of the charged particles, a 2 Tesla solenoidal magnet is installed surrounding the central tracking system. The central tracker has an excellent position resolution of 20-30 microns in the plane transverse to the beam direction. $\mathrm{D} \varnothing$ calorimeter is a sampling calorimeter with Uranium (absorber) and Liquid Argon (active material) for measuring the energies of the particles. It is a hermetic calorimeter with a pseudorapidity $^{\dagger}$ coverage of 4.2. Electrons/photons and hadrons lose energy in the calorimeter through different mechanisms and show different shower ${ }^{\ddagger}$ profiles. The $\mathrm{D} \varnothing$ calorimeter is capable of identifying both types of particles and measures the energies of the particles with good resolution. The muon detector consists of magnetic toroids, surrounded by three layers of drift chambers and scintillation counters. The drift chambers measure the trajectories of the muons before and after they traverse the magnetic irons; thus the momenta of the muon trajectories are measured. The scintillation counters are utilized to distinguish between cosmic muons and the ones coming from $p \bar{p}$ interactions. At Tevatron a large number of the $p \bar{p}$ collisions result in inelastic collisions and it is not feasible to record all of them. Rare physics processes like $t \bar{t}$ production are of great interest and must be recorded. To

\footnotetext{
${ }^{\dagger}$ pseudorapidity $\eta \simeq-\ln \tan \frac{\theta}{2}, \theta$ being the angle of the particle direction with beam direction (conventionally taken as $\bar{p}$ direction).

¥A high energy electron/photon produces a cascade of electron and positron pair through Bremstrahlung radiation; this is called electromagnetic shower. On the other hand, the hadrons lose energy through inelastic collisions with atomic nuclei producing secondary hadrons and thus result in hadronic showers.
} 
select only the physics processes of interest, $D \varnothing$ has a tri-level trigger system. The trigger system effectively brings down the interaction rate from $\sim 2.5$ $\mathrm{MHz}$ (the real $p \bar{p}$ collision rate) to $50 \mathrm{~Hz}$ for tape storage by hardware (at level 1 and level 2) and software (level 3) filtering. It is to be noted that, several triggers have been designed in the trigger system to select different physics processes. As the cross-sections vary from one physics process to another, the exposure rates ${ }^{\ddagger}$ for different triggers are also varied accordingly. Finally, the events are reconstructed ${ }^{\dagger}$ using different algorithms and analyzed offline. A full description of DØ detector can be found in Ref. [4].

\section{Data}

The signature of $t \bar{t} \rightarrow W W b \bar{b} \rightarrow e \nu_{e} \mu \nu_{\mu} b \bar{b}$ process leads to a pair of high $p_{T}$, isolated electron and muon, large missing transverse energy $\left(E_{T}\right)$ from the neutrinos and two high $p_{T}$ jets from $b$ quarks. Apart from the $e \mu$-decay of $t \bar{t}$ (signal), many other physics processes (backgrounds) can also result in the said signature; these backgrounds are detailed in the next section. In D $\varnothing$ trigger system, one of the triggers has been designed to select the events with an electron and a muon ( $e \mu$ trigger $)$; the events which are passed by this particular trigger are considered for the current analysis. As mentioned earlier, the data selected by this trigger corresponds to an integrated luminosity of $228.29 \mathrm{pb}^{-1}$. Various physics processes have different efficiencies for $e \mu$ trigger, under consideration; they are estimated using the Monte Carlo events. The Monte Carlo event simulation for signal and background processes are detailed in section 4 .

\footnotetext{
${ }_{\ddagger}^{\ddagger}$ Application of a particular trigger at a selective rate without any loss for rare events. ${ }^{\dagger}$ collating the information from different sub detectors in a certain manner
} 
Every physics process has its own features which help to distinguish it from other physics processes - as for example, the jets produced for most of the $e \mu$ background processes have lower energy than the jets from $t \bar{t}$ decays (as they arise from the decay of very massive particles). So, selecting the jets with a reasonably high $p_{T}(\geq 20 \mathrm{GeV})$ improves the background rejection. In addition, the sum of transverse energy of the jets and the transverse energy of the highest $p_{T}$ lepton (either electron or muon) is found to be a good discriminating variable between the signal and the background. Few more offline selection criteria have been tried to separate the signal from the backgrounds and the chosen cuts itemized in Table, are found to be the optimal combination in terms of the sensitivity of $t \bar{t}$ cross-section measurement.

\begin{tabular}{l}
\hline \hline One track-matched isolated electron with $P_{T}^{e}>15 \mathrm{GeV}$ and $\left|\eta_{\text {det }}^{e}\right|<2.5$ \\
$\&$ \\
One track-matched, isolated muon with $P_{T}^{\mu}>15 \mathrm{GeV}$ and $\left|\eta_{\text {det }}^{\mu}\right|<2.0$ \\
\hline$+\Delta R(e, \mu)>0.25$ \\
\hline$+\geq 2$ jets with $E_{T}^{\text {Jet }}>20 \mathrm{GeV}$ \\
\hline$+E_{T}>25 \mathrm{GeV}$ \\
\hline$+\Delta \phi\left(\mu, E_{T}\right)>0.25$ \\
\hline$+H_{T}^{\mathrm{Leading} \text { lepton }>140 \mathrm{GeV}}$ \\
\hline \hline
\end{tabular}

Table 1: Event selection criteria.

\section{Signal \& background}

A large fraction of $t \bar{t} \rightarrow e \mu$ events will appear to be undetected due to the detector acceptance and inefficiencies in event reconstruction. Moreover the application of offline selection criteria (vide Table ) would reduce 
the number by another factor. Thus the total detection efficiency is the product of kinematic efficiency, acceptance and reconstruction efficiency. To estimate the signal efficiency in $e \mu$ channel, a $t \bar{t} \rightarrow$ dilepton Monte Carlo (MC) sample generated using Alpgen [5] v1.2 (CTEQ 6.1M) and processed through Pythia [6] v6.2 (CTEQ5L) for fragmentation and decay. EvtGen [7] is used to model the decays of $b$ hadrons. The top quark mass is taken to be $175 \mathrm{GeV} / c^{2}$. The Monte Carlo sample contains all dilepton final states, including $\tau$ 's, with inclusive $\tau$ lepton decay. Tau leptons are decayed using TAUOLA [8]. These high $p_{T}$ events (hard scattered) are passed through a detailed detector silumation based on GEANT 3 [9]. In a $p \bar{p}$ bunch crossing at Tevatron, a hard scattered process can take place along with one or more low $p_{T}$ scattering processes (minimum bias) and the number of such minimum bias events in a $p \bar{p}$ bunch crossing, are found to be Poisson distributed. On average, 0.8 such events are overlaid on a hard scattered event. The events are then reconstructed using certain algorithm for offline comparison with data. Total signal efficiency for $t \bar{t} \rightarrow e \mu$ final state where electron and muon originate from a $W$ directly, or from the leptonic decay of a $\tau$ which itself comes from a W has been estimated to be $10.35 \%$. Several scale factors have been applied to rectify ${ }^{\ddagger}$ the $\mathrm{MC}$ efficiencies.

The $e \mu$ backgrounds can be classified into the following categories and they are estimated either from MC samples or from data as described below. Basically two types of events have been simulated for the major backgrounds (viz., $Z^{0} / \gamma^{*} \rightarrow \tau \bar{\tau} \rightarrow e \mu \nu_{e} \nu_{\mu} \nu_{\tau} \overline{\nu_{\tau}}$ and $W W \rightarrow e \mu$ ) which survive after applying all selection criteria. For comparison at the inclusive levels (i.e. without

\footnotetext{
${ }^{\ddagger}$ Data and $\mathrm{MC}$ efficiencies differ from each other due to various reasons. Taking the ratios of efficiencies between the $Z^{0}$ data and $Z^{0} \mathrm{MC}$, the $\mathrm{MC}$ efficiencies are rectified.
} 
any explicit requirement for jets), the events are generated with Pythia and for comparison at levels with $\geq 2$ jets requirement, the events are generated with Alpgen. In both cases, fragmentation and decay have been done using Pythia. These two types of MC events are referred as "Pythia" and "Alpgen" samples in the description of the backgrounds below. Finally as described for $t \bar{t}$ Monte Carlo above, these events are passed through detailed detector silumation, addition of minimum bias events and event reconstruction.

- Physics Backgrounds are the backgrounds where the final state contains a real electron and a real muon.

$-Z^{0} / \gamma^{*} \rightarrow \tau \bar{\tau} \rightarrow e \mu \nu_{e} \nu_{\mu} \nu_{\tau} \overline{\nu_{\tau}}$ is the dominating background for $t \bar{t} \rightarrow e \mu$ analysis at the production level. But due to the fact that the final state leptons (e and $\mu$ ) and the neutrinos come from the decay of $\tau$ 's, they are of lower $p_{T}$ compared to leptons coming from $t \bar{t}$ decay; moreover the jets in these events are also of lower energies when compared with the jets from $t \bar{t}$. So effectively, this background gets rejected by almost all the cuts tabulated in Table. Both Alpgen and Pythia samples have been utilized to study this background.

$-\boldsymbol{W}^{+} \boldsymbol{W}^{-} \rightarrow \boldsymbol{e} \boldsymbol{\mu}$ events are very similar to $t \bar{t} \rightarrow e \mu$ events in both lepton and neutrino $p_{T}$ 's, apart from the fact that the jets in $t \bar{t}$ are much harder in $p_{T}$. Therefore the requirement for $\geq 2$ jets (with $P_{T}>20 \mathrm{GeV}$ ) eliminates $W^{+} W^{-}$background to a great extent. Pythia and Algen samples of $W^{+} W^{-} \rightarrow e \mu$ have been studied in great detail for understanding this background.

$-\boldsymbol{W}^{ \pm} \boldsymbol{Z}^{0} \rightarrow \boldsymbol{\mu} \nu_{\mu} e^{+} \boldsymbol{e}^{-} / e \boldsymbol{\nu}_{e} \boldsymbol{\mu}^{+} \boldsymbol{\mu}^{-}$is a tiny background at the pro- 
duction level and has almost negligible contribution $(0.002 \pm 0.000)$ at the final state after applying all cuts. Pythia MC is used to estimate the effective event yields at different cut levels.

- Photon Backgrounds contribute to the $e \mu$ analysis where the events contain a muon and a photon, misidentified as an electron. There are two types of photons which can come into the $e \mu$ analysis as described below.

- Muon Bremsstrahlung: A muon can radiate a bremsstrahlung photon in the detector and the photon can get misidentified as an electron in both tracker and calorimeter. Here the direction of the misidentified electron in transverse plane would be very close to the muon direction and the $\Delta R(e, \mu)^{\dagger}$ cut in Table, reduces these backgrounds by a significant amount. There are following two such physics processes which can result in above scenario.

$* \boldsymbol{W} \rightarrow \boldsymbol{\mu} \boldsymbol{\nu}_{\boldsymbol{\mu}}$ gets completely rejected by the $\Delta R(e, \mu)$ cut. To check the consistency with data observation, Pythia events are generated to calculate the event yields at the inclusive level.

$* \boldsymbol{Z}^{0} / \boldsymbol{\gamma}^{*} \rightarrow \boldsymbol{\mu}^{+} \boldsymbol{\mu}^{-}$events survive even after applying the $\Delta R(e, \mu)$ cut when the muon (emits the bremsstrahlung photon) does not get reconstructed in the muon chambers. In this case, the photon results in an EM cluster in the calorimeter and gets associated to an isolated track which originally

\footnotetext{
$\dagger^{\dagger} R=\sqrt{\Delta \eta^{2}+\Delta \phi^{2}}$
} 
belongs to the lost high $p_{T}$ muon; thus becomes a perfect electron candidate. Pythia samples are extensively used to estimate the contributions for this background.

- Associated photon production: On contrary to the bremsstrahlung photons, these photons are produced in the hard-scattered events. Here the photon either gets associated to a random track or gets converted into an $e^{+} e^{-}$pair in the tracker and one of them $\left(e^{+}\right.$or $\left.e^{-}\right)$appears as an electron candidate for the $e \mu$ analysis. The associated production of $\boldsymbol{W}\left(\rightarrow \boldsymbol{\mu} \boldsymbol{\nu}_{\boldsymbol{\mu}}\right)$ or $\boldsymbol{Z}^{0}\left(\rightarrow \boldsymbol{\mu}^{+} \boldsymbol{\mu}^{-}\right)$ along with a photon are the sources of these backgrounds. Pythia events have been generated to study these backgrounds.

- Instrumental Backgrounds are the events where the leptons (either electron or muon or both) originate from the jets. In case of muon, it comes from the semileptonic decay of heavy quarks and appears to be an isolated muon. On the other hand for electron, it can be either a semileptonic decay of heavy quark or a misidentification of a jet in both calorimeter and tracker. $\boldsymbol{W}\left(\rightarrow \boldsymbol{\mu} \boldsymbol{\nu}_{\boldsymbol{\mu}}\right)+$ jets, $\boldsymbol{W}\left(\rightarrow \boldsymbol{e} \boldsymbol{\nu}_{\boldsymbol{e}}\right)+$ jets and QCD multijet processes may result in $t \bar{t} \rightarrow e \mu$ topology when one of the jets produces fake lepton $(e / \mu)$ signal. Contributions for these backgrounds are directly measured from experimental data.

\section{Calculation of Cross section}

In order to estimate the cross section, a maximum likelihood method has been utilized. Poisson hypothesis is considered to account for the small number of observed events. The likelihood of $N^{\text {Obs }}$ events, given the hypoth- 
esis of $\tilde{N}\left(\equiv \sigma \cdot B R \cdot L^{\text {integrated }} \cdot \epsilon+N^{\mathrm{bkg}}\right)$ is defined as:

$$
\mathcal{L}\left(\sigma,\left\{N^{\mathrm{Obs}}, N^{\mathrm{bkg}}, \mathrm{BR}, L^{\text {integrated }}, \epsilon\right\}\right)=\frac{\tilde{N}^{N^{\mathrm{Obs}}}}{N^{\mathrm{Obs}}} \cdot e^{-\tilde{N}}
$$

(where $N^{\mathrm{Obs}}, N^{\mathrm{bkg}}, L^{\text {integrated }}, B R$ and $\epsilon$ are respectively the number of observed events, the number of background events, integrated luminosity, the branching fraction of $t \bar{t} \rightarrow e \mu$ (including $\tau$-contributions) and the overall signal efficiency.)

The cross section is then estimated by the minimization of

$$
-2 \ln \mathcal{L}\left(\sigma,\left\{N^{\mathrm{Obs}}, N^{\mathrm{bkg}}, \mathrm{BR}, L^{\text {integrated }}, \epsilon\right\}\right)
$$

and is given by:

$$
\sigma(p \bar{p} \rightarrow t \bar{t})=\frac{N^{\text {Obs }}-N^{\text {bkg }}}{L^{\text {integrated }} \cdot \epsilon \cdot B R(t \bar{t} \rightarrow e \mu)}
$$

\section{Results}

After applying all selection criteria, the expected number of signal and background events are shown in Table. The systematic uncertainty on the cross section measurement is obtained by varying the background and the signal efficiencies within their errors. As mentioned in Section 4, the overall signal efficiency is determined to be $10.20 \%$ and $t \bar{t} \rightarrow e \mu$ has the branching ratio of $3.16 \%[2]$. The $\mathrm{D} \varnothing$ luminosity measurement has $6.5 \%$ relative uncertainty and is considered to be uncorrelated from systematics. 


\begin{tabular}{r|c|c|c}
\hline \hline Category & Yield & Stat Err & Sys Err \\
\hline$Z / \gamma^{*} \rightarrow \tau \tau$ & 0.35 & \pm 0.06 & \pm 0.07 \\
$W W \& \mathrm{WZ}$ & 0.35 & \pm 0.00 & $+0.17-0.14$ \\
$\gamma$-processes & 0.02 & \pm 0.02 & $+0.01-0.02$ \\
Fakes (QCD, $W \rightarrow e \nu, W \rightarrow \mu \nu)$ & 0.19 & \pm 0.02 & \pm 0.06 \\
\hline Total background & 0.91 & \pm 0.07 & $+0.20-0.17$ \\
\hline Expected Signal & 5.14 & \pm 0.11 & $+0.57-0.47$ \\
\hline Selected Events & 8 & & \\
\hline \hline
\end{tabular}

Table 2: Expected background and observed and expected signal yields. The expected signal yield assumes a $7 \mathrm{pb} t \bar{t}$ production cross section based on theoretical calculations $[10,11]$.

The $t \bar{t}$ production cross section at $\sqrt{s}=1.96 \mathrm{TeV}$ in $e \mu$ channel is measured to be:

$$
9.7_{-3.4}^{+4.3} \text { (stat) }{ }_{-1.3}^{+1.2} \text { (sys) } \pm 0.6 \text { (lumi) pb }
$$

The likelihood fuction is utilized to derive the statistical uncertainties on cross section measurement.

\section{Conclusions}

The latest theoretical calculations can be obtained from Ref. [10, 11]. The $t \bar{t}$ production cross section has been measured in $e \mu$ channel at $\sqrt{s}=1.96$ $\mathrm{TeV}$ and is consistent with Standard Model prediction. 


\section{Bibliography}

[1] S. W. Herb et al., Phys. Rev. Lett., 39, 252 (1977).

[2] S. Eidelman et al., Phys. Lett. B, 592, 1 (2004).

[3] DØ Collaboration, V. M. Abazovet al., Phys. Rev. D, 67, 012004 (2003).

[4] URL: http://www-d0.fnal.gov/detector/

[5] M.L. Mangano, M. Moretti, F. Piccinini, R. Pittau, A. Polosa, JHEP, 0307, 1 (2003);

hep-ph/0206293; M.L. Mangano, M. Moretti, R. Pittau, Nucl. Phys. B, 632, 343 (2002); hep-ph/0108069; F. Caravaglios, M. L. Mangano, M. Moretti, R. Pittau, Nucl. Phys. B, 539, 215 (1999); hep-ph/9807570.

[6] T. Sjöstrand, P. Edén, C. Friberg, L. Lönnblad, G. Miu, S. Mrenna and E. Norrbin, Comp. Phys. Commun., 135, 238 (2001).

[7] D. Lange, A. Ryd et al., The EvtGen Event Generator Package, in Proceedings of CHEP (1998).

[8] S. Jadach, Z. Was, R. Decker, J.H. Kuehn, The tau decay library TAUOLA: version 2.4 QED corrections in decay modes, Comp. Phys. Commun., 76, 361 (1993); CERN TH-6793 preprint.

[9] R. Brun and F. Carminati, CERN program library long writeup, W5013 (1993).

[10] N. Kidonakis et. al., hep-ph/0303186 (2003)

[11] M. Cacciari et. al., hep-ph/0303085 (2003) 


\title{
List of Publications
}

\author{
- In Conference
}

- Combined t $\overline{\mathrm{t}}$ Production Cross Section at $\sqrt{\mathrm{s}}=1.96 \mathrm{TeV}$ in the Lepton+jets and Dilepton Final States using Event Topology URL: http://www-d0.fnal.gov/Run2Physics/top/top_public_web_pages/conference_notes/summer05_xsec_lj-11_combined_confnote.pdf

\section{- In Journal}

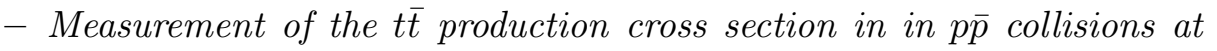
$\sqrt{s}=1.96$ TeV in dilepton final states, D $\varnothing$ collaboration, Phys. Lett. B 626, 55 (2005); hep-ex/0505082

\section{- DØ Internal Document}

- Measurement of the $t \bar{t}$ Production Cross-section at $\sqrt{s}=1.96 \mathrm{TeV}$ in Dilepton Final States - DØ note 4623, Version 1.6, February 21, 2005; Analysis approval on February 28, 2005, by DØ Internal Review Committee for submission to Phys. Lett. B 



\section{Acknowledgement}

Words of appreciation are not enough to express my gratitude to numerous people who cooperated with me for this thesis work directly or indirectly, and to those who envisioned my life beyond a graduate student.

First I would like to express my thanks and gratitude to my adviser, Sudeshna Banerjee for her invaluable guidance and constant support to the work, presented in this dissertation. Her practical and straightforward approach to every problem has set an example for me. Besides her, I owe to Naba K. Mondal and V. S. Narsimham who helped me to understand various aspects of high energy physics. I convey my grateful thanks to

Sunanda Banerjee, B. S. Acharya, Gobinda Majumdar, Manoranjan Guchait and Shashi Dugad, for many academic and social discussions, during my graduate school at TIFR.

It is a great experience of mine, to have availed the spectacular research facilities, maintained at TIFR. I duly acknowledge "TIFR Alumni Association Scholarship for Career Development", offered as an encouragement towards my research and training pursuits at TIFR graduate school.

I am thankful to my colleagues and members of high energy physics group at TIFR for providing me an excellent work atmosphere. I express my heartfelt thanks to my batch-mates and numerous friends at TIFR, for many enlightening discussions and invaluable company, over last five years. I will bear the cherished memories of friendship with Rituparno, Ramanan and Parinda, who enriched my life as a graduate student. Besides them, Subham, Sourin, Subhendu, Debanjan, Satya and Pratap merit special men- 
tion for their heartiest cooperation at the personal front. Thanks to Partha and Anandamohan for all fun and frolic in TIFR ghetto, over last couple of months.

Working under the colourful fabric of D $\varnothing$ has been a remarkable experience to remember. I am indebted to Aurelio Juste and Arnulf Quadt, for their sincere coordination and diligence as Top Physics group conveners. I extend my words of appreciation to Christophe Clément, Bob Kehoe and Elizaveta Shabalina, for their continuous attention to the nattiest details of the analysis, presented in this dissertation. As a member of extended top production sub-group, I take the opportunity to thank my colleagues, Joe Kozminski, Stefan Anderson, Tobias Golling, Daniel Whiteson, Markus Klute and Jessica Leveque. I owe a lot to the members of D $\varnothing$ luminosity ID group; specially to Michael Begel, Brendan Casey and Andrei Nemorotski. My special thanks are also due to my mentor Marco Verzocchi for teaching me the details of software programming, from the basics to $\mathrm{D} \varnothing$ framework. I am honoured to have a true "friend, philosopher and guide" like Suyong Choi during my stay at Fermilab. I express my profound thanks to Nirmalya, Dhiman and Supriya, who made my life enjoyable at Fermilab.

No words are enough to express my gratitude to my family members: my sister, my jethu and my wife, whose unwavering patience and diligence for me all along my Ph.D. career can ever be forgotten in life! 


\section{List of Figures}

1.1 Four types of fundamental forces between a typical pair of particles. . . . . 8

1.2 Patterns of charge-changing weak transitions among quarks and leptons. The strongest inter-quark transitions correspond to the solid lines, with dashed, dot-dashed, and dotted lines corresponding to successively weaker transitions. . . . . . . . . . . . . . . . . 10

1.3 The strong-coupling constant $\alpha_{S}\left(M^{2}\right)$ as a function of energy scale (M), subject to the constraint $\alpha_{S}\left(M_{Z}^{2}\right)=0.118 \pm 0.002$. The solid line shows the central value; dashed lines indicate $\pm 1 \sigma$ limits. . . . . . . . . . . . . 13

1.4 Typical effect of injecting energy into a hadron to separate the quarks: (a) original quark -antiquark pair held by colour lines of force; (b) lines of force are stretched; (c) lines of force break with the creation of a new quark-antiquark pair. . . . . . . . . . . . . . . . . . . . 14

1.5 $\mathrm{CDF}$ and $\mathrm{D} \varnothing$ cross section results for $t \bar{t}$ production at the Fermilab Tevatron, Run I (with $\sqrt{s}=1.8 \mathrm{TeV}$ ), overlaid with the theory prediction. For the latter, the entire band includes the NLO+NLL and partial NNLO+NNLL predictions. . . . . . . . . . . . . . . . . . 17

1.6 Comparison of $\mathrm{CDF}$ and $\mathrm{D} \varnothing$ measurements of the top quark mass at the Fermilab Tevatron, Run I (with $\sqrt{s}=1.8 \mathrm{TeV}$ ) [21] . . . . . . . . . . 18

1.7 Electroweak radiative corrections to the $W$ boson propagator: these corrections are proportional to $m_{t}^{2}$ and $\ln m_{H} \ldots \ldots \ldots \ldots$. . . . . . . . 21

1.8 The comparison of the indirect measurements of $M_{W}$ and $M_{t}$ (LEP I+ SLD data) (solid contour) and the direct measurements ( $p \bar{p}$ colliders and LEP II data) (dashed contour). In both cases the $68 \%$ CL contours are plotted. Also shown is the Standard Model relationship for the masses as a function of the Higgs mass. The arrow labeled $\Delta \alpha$ shows the variation of this relation if $\alpha\left(M_{Z}^{2}\right)$ is changed by one standard deviation. This variation gives an additional uncertainty to the Standard Model band shown in the figure $[$ hep/ex-0312023]. . . . . . . . . . . . . . . . . . . . . . . 22

1.9 Feynman diagrams of pair production of top quarks at hadron colliders. . . 23

1.10 Parton distribution functions of a proton at the scale $\mu=m_{t}$, relevant for top-quark production. The probability of finding a given parton species with momentum fraction between $x\left(=x_{a} \approx x_{b}=x\right.$ where $\left.x \approx \frac{2 m_{t}}{\sqrt{s}}\right)$ and $x+d x$ is $f(x) d x \ldots \ldots \ldots \ldots \ldots \ldots \ldots \ldots \ldots$ 
1.11 The $t \bar{t}$ production cross section (a) as a function of top quark mass at $\sqrt{s}=1.96 \mathrm{TeV}$, evaluated at a scale $\mu=m_{t}$, (b) as a function of $\mu / m_{t}$, for $m_{t}=175 \mathrm{GeV} / \mathrm{c}^{2}$. The difference in the choice of kinematics viz., PIM, 1PI is also shown (see Ref. [30]). . . . . . . . . . . . . . . . . 28

1.12 Feynman diagram for $t \rightarrow b W^{+}$from which the decay width of the top quark $\left(\Gamma_{\mathrm{t}}\right)$ can be calculated to leading order. . . . . . . . . . . . . 29

1.13 Breakdown of $t \bar{t}$ events into various final states and their branching fractions. 32

1.14 The schematics of various $t \bar{t} \rightarrow e \mu$ decays: (a) $t \bar{t} \rightarrow W^{+} b W^{-} \bar{b} \rightarrow e \nu_{e} \mu \nu_{\mu} b \bar{b}$, (b) $t \bar{t} \rightarrow W^{+} b W^{-} \bar{b} \rightarrow e \nu_{e} \tau \nu_{\tau} b \bar{b}\left(\mu \nu_{\mu} \tau \nu_{\tau} b \bar{b}\right) \rightarrow e \nu_{e} \mu \nu_{\mu} \nu_{\tau} b \bar{b}\left(\mu \nu_{\mu} e \nu_{e} \nu_{\tau} b \bar{b}\right),(\mathrm{c})$ $t \bar{t} \rightarrow W^{+} b W^{-} \bar{b} \rightarrow \tau \nu_{\tau} \tau \nu_{\tau} b \bar{b} \rightarrow \mu \nu_{\mu} \nu_{\tau} e \nu_{e} \nu_{\tau} b \bar{b} \ldots \ldots \ldots \ldots \ldots$

1.15 Leading order diagram for $Z^{0} / \gamma^{*} \rightarrow \tau^{+} \tau^{-}$at Tevatron. . . . . . . . . . 39

1.16 Leading order diagram for $W^{+} W^{-}$production at Tevatron: (a) s-channel and (b) t-channel. . . . . . . . . . . . . . . . . 39

2.1 Tevatron accelerator complex at Fermilab with two collider detectors viz., $\mathrm{CDF}$ and $\mathrm{D} \emptyset \ldots \ldots \ldots \ldots \ldots \ldots \ldots \ldots$

$2.2 \mathrm{H}^{-}$source: atoms of gaseous hydrogen gets ionised into a proton and an electron under the electric field and the proton congregates on metal surface. Sometimes a proton (drifting towards Cesium surface) smacks a proton with two electrons off the Cesium surface. . . . . . . . . . . . . . . . . . 45

2.3 A layout of Antiproton source. . . . . . . . . . . . . . . . . . 48

2.4 A diagram showing the components in the Antiproton target station. . . . . 48

2.5 Stochastic Cooling of antiprotons, in Debuncher and Accumulator. . . . . . 49

2.6 A cross-sectional view of the $\mathrm{D} \varnothing$ detector, showing different components. . 54

2.7 The coordinate systems showing the polar angle $(\theta)$, radial distance $(\mathrm{r})$ and azimuthal angle $(\phi) \ldots \ldots \ldots \ldots \ldots \ldots \ldots$

2.8 Schematic diagram of $\mathrm{D} \varnothing$ luminosity monitors: (a) 3-D layout of two sets of counters, (b) cross sectional view of each monitor (circular spots on each wedge are the places where the PMT's are mounted). . . . . . . . . . . 58

2.9 Cartoon diagram showing the particles from (a) proton/antiproton halos, (b) $p \bar{p}$ collision. . . . . . . . . . . . . . . . . . . . . . . 59

2.10 Schematic diagram showing the layout of the DØ Central Tracking System. 61

2.11 Layout showing different parts of the Silicon Microstrip Tracker. . . . . . . 62

2.12 End view of the barrel structure in SMT. . . . . . . . . . . . . 63

$2.13 r-\phi$ view of an F-disk in SMT, showing the wedges of silicon detectors. . . 64

2.14 Diagram showing the geometry of CFT: a) Cut view of showing a quarter of the detector in $r-z$ with 8 super-layers b) Cross sectional view of the fibres within a ribbon in $r-\phi . \ldots \ldots \ldots 6 \ldots$

2.15 Impact parameter resolution, $\sigma\left(d_{0}\right)$ of the Central Tracker as a function of track $p_{T} \ldots \ldots \ldots \ldots \ldots \ldots \ldots \ldots$

2.16 Schematic side and end views of $\mathrm{D} \varnothing$ Central Preshower detector. . . . . . . 70

2.17 The cross section of a layer of the CPS, showing the dimension of each unit. The triangles are made of extruded plastic scintillator and the circles in the middle contain WLS fibres. Each triangular strip is wrapped in reflective material to increase light yield and reduce cross talk. . . . . . . . . . . 72

2.18 Diagram showing a y-z view of the FPS detector. . . . . . . . . . . . . 74

2.19 An isometric view of the $\mathrm{D} \varnothing$ calorimeter. . . . . . . . . . . . 75 
2.20 Typical unit cell in DØ sampling calorimeter. . . . . . . . . . . . . . . . 79

2.21 One quarter section view of the $\mathrm{D} \varnothing$ calorimeters showing the segmentation

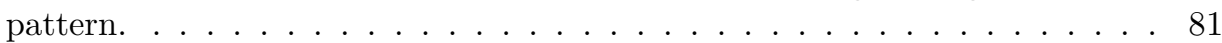

2.22 A cut-away view of the D $\varnothing$ Muon system. . . . . . . . . . . . . . 87

2.23 Extruded aluminum sections from which the $\mathrm{B}$ and $\mathrm{C}$ layer PDT chambers are constructed. The A layer chamber extrusions are similar, but have four cells instead of three. The 'x' marks the position of the sense wire. . . . . . 88

2.24 WAMUS pads viewed from the top. . . . . . . . . . . . . . . . . . 89

2.25 Design of A-layer MDT's. . . . . . . . . . . . . . . . . . . . . 91

2.26 A 8-cell unit of MDT (dimensions are in the units of millimetre.). . . . . . . 91

2.27 A simulated muon trajectory through $\mathrm{D} \varnothing$ detector. . . . . . . . . . . . . 92

2.28 Local muon momentum resolution as a function of muon momentum, $p$ for central (a) and forward (b) regions. . . . . . . . . . . . . . . 93

2.29 Schematic illustration of $\mathrm{D} \varnothing$ tri-level trigger system. . . . . . . . . . . . . 95

2.30 Block diagram illustrating the components of Level 1 and Level 2, and the connections between them. ...................

3.1 Illustration of Histogramming Track Finder with a single track with $p_{T}=$ $1.5 \mathrm{GeV}$, consisting of 5 hits.(a) a set of trajectories in coordinate space with a given hit, originating from the geometric centre of the detector; (b) a straight line in parameter space describing all possible trajectories from a single hit. (c) 5 such straight lines in parameter space, from the 5 hits; the point of intersection of these straight lines corresponds to the track parameters. (d) The point of intersection corresponds to a peak in a 2 dimensional histogram in $\left(\phi, \rho_{0}\right) \ldots \ldots$. . . . . . . . . . . . . 108

3.2 Illustration of the different variables to reconstruct initial track hypothesis out of three hits in AAtrack algorithm. . . . . . . . . . . . . . . . 109

3.3 Expansion of track hypothesis, built out of three initial hits to the other layers of tracker, in AAtrack algorithm. . . . . . . . . . . . . . . . 109

3.4 The distributions for the input variables to the 7-parameter EM Likelihood in CC - electron (black) and background (red): (a) EM fraction, (b) Hmx7, (c) $E_{T}^{\text {Cal }} / p_{T}^{\text {track }},\left(\right.$ d) Logarithm of Prob $\left(\chi_{\text {Spatial EM-trk }}^{2}, 2\right)$, (e) Distance of closest approach of the candidate track, (f) Number of tracks in a $\Delta R<0.05$ cone around the candidate track and $(\mathrm{g}) \sum p_{T}^{\text {track }}$ in a $\Delta R<0.4$ cone around the candidate track. . . . . . . . . . . . . . . . . . . . . 124

3.5 The distributions for the input variables to the 7-parameter EM Likelihood in EC - electron (black) and background (red): (a) EM fraction, (b) Hmx7, (c) $E_{T}^{\text {Cal }} / p_{T}^{\text {track }},(\mathrm{d})$ Logarithm of Prob $\left(\chi_{\text {Spatial EM-trk }}^{2}, 2\right),($ e) Distance of closest approach of the candidate track, (f) Number of tracks in a $\Delta R<0.05$ cone around the candidate track and $(\mathrm{g}) \sum p_{T}^{\text {track }}$ in a $\Delta R<0.4$ cone around the candidate track. . . . . . . . . . . . . . . . . 125

3.6 The 7-parameter EM likelihood distributions for electron (black) and background (red) in $\mathrm{CC}(\mathrm{a})$ and $\mathrm{EC}(\mathrm{b})$; the background efficiency vs electron efficiency for different thresholds of EM Likelihood in $\mathrm{CC}(\mathrm{c})$ and $\mathrm{EC}(\mathrm{d})$. . . 127

3.7 Energy loss (shown as, EMC-E) of $20 \mathrm{GeV}$ electron as a function of $\left(\phi^{\prime}+0.1\right)$ (see text); the edge of the module is around $0.1 . \ldots . . . . . . . . .130$ 
3.8 (a) Parabola fit of the energy loss (shown as EMC-E) of $5 \mathrm{GeV}$ electrons as a function of $\eta_{p}$; (b) Variation of the fit parameters, etacorra (top), etacorrb (middle), etacorrc (bottom) as a function of electron energy. . . . . . . . 131

3.9 EM energy correction factor $\epsilon$ as functions of (a) $\eta$ in CC, (b) $\phi$ in CC, (c) $\phi$ in North EC and, (d) $\phi$ in South EC (d). . . . . . . . . . . . . 133

3.10 A halo cone $\mathrm{R}$, with inner edge $R_{a}$ and outer edge $R_{b}$ surrounding the muon. The transverse energies of all calorimeter cells i at a radius $R_{i}$ with $R_{b}>R_{i}>R_{a}$ where $R_{a}\left(R_{b}\right)=0.1(0.4) \ldots \ldots \ldots \ldots \ldots \ldots$

3.11 An illustration of the different levels of jet definition. . . . . . . . . . . 141

3.12 The total jet energy scale correction and its uncertainty for data, as a function of uncorrected jet energy for a jet at $\eta=0.0$ (top) and as a function of jet $\eta$ for a jet of $E_{\text {jet }}=50.0 \mathrm{GeV}$ (bottom). The displayed uncertainties are statistical and total (statistical and systematic errors are added in quadrature). . . . . . . . . . . . . . . . . . . . . . . . 152

3.13 The uncertainties are displayed separately for the offset, response and showering corrections, and for the total correction as a function of $E_{T}$ (top) and $\eta$ (bottom) of the jets (in each case, statistical and systematic errors are added in quadrature). . . . . . . . . . . . . . . . . . . . . 153

3.14 The total jet energy scale correction and its uncertainty for Monte Carlo, as a function of uncorrected jet energy for a jet at $\eta=0.0$ (top) and as a function of jet $\eta$ for a jet of $E_{j e t}=50.0 \mathrm{GeV}$ (bottom). The displayed uncertainties are statistical and total (statistical and systematic errors are added in quadrature). . . . . . . . . . . . . . . . . . . 15

4.1 The integrated luminosity for Tevatron Run II from April 2002 to August

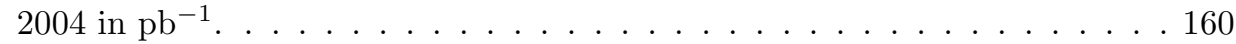

4.2 The shift (a) and RMS (b) of the missing transverse energy per run, as a function of the run serial number. The average (c) of the scalar transverse energy per run as a function of the serial run number. . . . . . . . . . 163

4.3 Level 1 electron trigger efficiencies as a function of $p_{T}$ of the electron from two different samples. (a) The sample with smaller statistics, shows that the Level 1 electron trigger becomes $100 \%$ efficient around $p_{T} \sim 10 \mathrm{GeV}$; (b) a sample with large statistics having offline electrons with $p_{T} \geq 15 \mathrm{GeV}$, shows that Level 1 electron trigger has $100 \%$ efficiency for electrons with

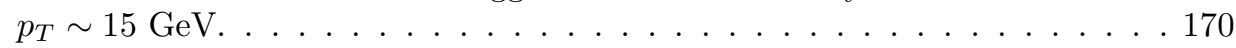

4.4 Level 1 muon trigger efficiencies as a function $\eta$ for mu1ptxatxx. . . . . . 171

4.5 Probabilities for a jet to fire Level 1 electron trigger as a function of $p_{T}$. . 173

4.6 Probabilities for a jet to fire Level 3 electron trigger as a function of $p_{T}$. . 174

4.7 Distribution of di-electron invariant mass for data and $\mathrm{MC} Z^{0} / \gamma^{*} \rightarrow e e$ events, with a Gaussian fit, for the case (i) with both electrons in the CC and in the fiducial regions of the detector (top), with (ii) both electrons in the $\mathrm{CC}$ and any one electron not in the fiducial region (middle), and (iii) with both electrons in the EC (bottom). . . . . . . . . . . . 188

$4.8 M_{e e}$ distribution in ee events with zero or more jets. The smeared $Z^{0}$ Monte Carlo is compared with data containing two electrons. (a) shows the distribution in a range of $0-175 \mathrm{GeV}(\mathrm{b})$ shows the distribution in a range of $75-105 \mathrm{GeV}$. . . . . . . . . . . . . . . . . . . . 190 
4.9 CFT coverage . . . . . . . . . . . . . . . . . . . 191

4.10 Comparison of the dimuon invariant mass in data (markers) and smeared Monte Carlo (solid histogram) in the $Z^{0}$ mass window. . . . . . . . . . . . . 193

4.11 Jet $p_{T}$ resolutions for different $\eta_{\text {det }}$ regions in MC. The points below $\sim 50 \mathrm{GeV}$ are obtained using $\gamma+$ jet events, whereas for $p_{T}>50 \mathrm{GeV}$ resolutions are measured using dijet data. Bands of $\pm 1 \sigma$ statistical error are also shown. . 196

4.12 Jet $p_{T}$ resolutions for different $\eta_{\text {det }}$ regions in data. The points below $\sim 50 \mathrm{GeV}$ are obtained using $\gamma+$ jet events, whereas for $p_{T}>50 \mathrm{GeV}$ resolutions are measured using dijet data. Bands of $\pm 1 \sigma$ statistical error are also shown. . . . . . . . . . . . . . . . . . . 197

$5.1 \Delta \phi\left(\mu, \mathrm{E}_{\mathrm{T}}\right)$ vs $\mathrm{E}_{\mathrm{T}}$ distributions after $e, \mu$ selection and $\Delta R(e, \mu)>0.25$ cut (events with zero or more jets). Relative event density is shown in colours: (a) $t \bar{t}$, (b) $W^{+} W^{-}$, (c) fakes and (d) $Z^{0} / \gamma^{*} \rightarrow \tau^{+} \tau^{-}$. For $t \bar{t}$, the plot is equally populated, while the distributions are non-uniform for the background channels. $Z^{0} / \gamma^{*} \rightarrow \tau^{+} \tau^{-}$events produce significantly softer $\mathrm{E}_{\mathrm{T}}^{\mathrm{T}}$ spectrum as expected. . . . . . . . . . . . . . . . . 203

$5.2 \Delta \phi\left(\mu, \mathbb{E}_{\mathrm{T}}\right)$ vs $\mathbb{E}_{\mathrm{T}}$ distributions after applying all all cuts: (a) $t \bar{t}$, (b) $W^{+} W^{-}$, (c) fakes and (d) $Z^{0} / \gamma^{*} \rightarrow \tau^{+} \tau^{-} . t \bar{t}, W^{+} W^{-}$and fake distributions are quite uniform while for a significant fraction of $Z^{0} / \gamma^{*} \rightarrow \tau^{+} \tau^{-}$events, the $\mathrm{E}_{\mathrm{T}}$ direction is close to the muon direction. . . . . . . . . . . . . . . . 204

5.3 Values of number of signal $(S)$ vs. number of expected background $(B)$ in grid search (shown in yellow marker). Contours with $\sqrt{S+B} / S$ is shown. Best choice is indicated by "Lowest $\sqrt{S+B} / S$ ", while the "Operating point" corresponds to the cuts tabulated in Tab. 5.1. The sensitivity of the previous version of this analysis [89] is also shown and referred to as "Moriond".

5.4 Variation of $\sqrt{S+B+\Delta B_{\text {syst }}^{2}} / S$ with expected number of signal $(S)$ events.209

5.5 EM reconstruction and identification efficiencies in data and Monte Carlo and the corresponding scale factors as function of the distance between the electron track and the closest jet in CC (left) and EC (right). . . . . . . . . 215

5.6 Track match times likelihood efficiency as function of $\eta$ (left plots) and $\phi$ (right plots) in CC (upper plots) and EC (lower plots). . . . . . . . . . 218

5.7 Distance between the second highest $p_{T}$ muon and the closest jet in $Z^{0} / \gamma^{*}+$ jet candidate events, when the highest $p_{T}$ muon is isolated, is shown on left for data and Monte Carlo. The right plot shows the distance between the closest jet and the highest $p_{T}$ muon when isolation selections are applied to the second highest $p_{T}$ muon. . . . . . . . . . . . . . . . . . . . . . . . . 224

5.8 Ratio of the muon isolation efficiencies in data and Monte Carlo, measured in $Z^{0} / \gamma^{*}+$ jet events, as a function of the distance between the second highest $p_{T}$ muon and the closest jet (derived from Fig. 5.7 (a)). The result of the linear fit is a scale factor of $1.001 \pm 0.006$. . . . . . . . . . . . . . . 224

5.9 Scale factor for jet reconstruction $\times$ jet identification efficiency in $\mathrm{CC}, \mathrm{ICD}$ and EC with the associated $\pm 1 \sigma$ band used for the systematic uncertainty on the central value. . . . . . . . . . . . . . . . . 227 
$5.10 \Delta R(e, \mu)$ distribution in data (markers), and in $Z^{0} / \gamma^{*} \rightarrow \mu \mu, W^{ \pm} \rightarrow \mu \nu_{\mu}$, and $Z^{0} / \gamma^{*} \rightarrow \tau \tau$ processes, where an event consists of one tight electron and one tight isolated muon. It is to be noted that the Monte Carlo prediction at the first bin i.e., $\approx 42$ events, matches quite well with the data observation of $46 \pm 6.78$ events (see Tab. 5.17). . . . . . . . . . . . . . . . 233

5.11 Correlation of 'Rattrk' and 'Rat11' parameters of the muon, in the events with 'loose' and 'tight' electrons in (a) CC and (b) EC. The events containing one 'medium' muon and one 'loose' ('tight') electron are represented by the points with yellow (black) marker. The region at bottom left corner of each plot contains physics backgrounds. These events are removed by only accepting events with non-isolated muons in upper right quadrant of each

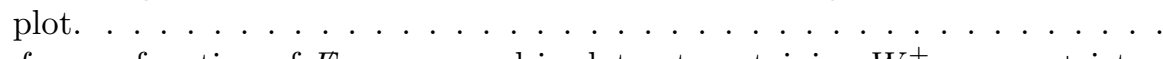

$5.12 f_{\mu}$ as a function of $\mathbb{E}_{T}$, measured in dataset containing $W^{ \pm} \rightarrow \mu \nu_{\mu}+$ jets and QCD events. . . . . . . . . . . . . . . . . . . 245

5.13 Distributions for detector $\eta$ (left) and $p_{T}$ (right) of electrons in $t \bar{t} \rightarrow e \mu$, background and data events having one good electron and one good muon

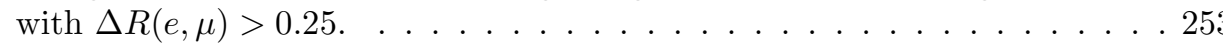

5.14 Distributions for $\eta$ (left) and $p_{T}$ (right) of muons in $t \bar{t} \rightarrow e \mu$, background and data events having one good electron and one good muon with $\Delta R(e, \mu)>$

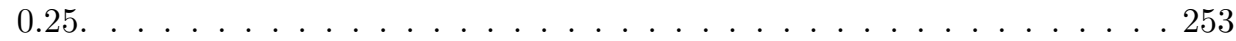

5.15 Distributions of (a) number of jets having $p_{T}>20 \mathrm{GeV}$ and (b) $p_{T}$ of the leading lepton, in $t \bar{t} \rightarrow e \mu$, background and data events having one good electron and one good muon with $\Delta R(e, \mu)>0.25 \ldots \ldots \ldots \ldots . \ldots 254$

5.16 Distributions of $\eta$ (left), $p_{T}$ (right) of the leading jet in $t \bar{t} \rightarrow e \mu$, background and data events having one good electron and one good muon with

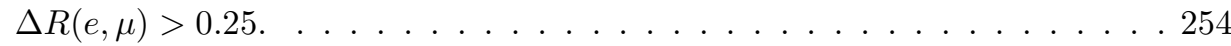

5.17 Distributions of the $E_{T}$ (left) and $\Delta \phi\left(\mu, E_{T}\right)$ (right) in $t \bar{t} \rightarrow e \mu$, background and data events having one good electron and one good muon with

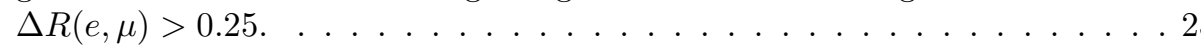

5.18 Distributions of the aplanarity in linear (left) and $\log$ (right) scale in $t \bar{t} \rightarrow$ $e \mu$, background and data events having one good electron and one good muon with $\Delta R(e, \mu)>0.25 \ldots \ldots \ldots \ldots \ldots \ldots \ldots \ldots \ldots \ldots$

5.19 Distributions of the sphericity in linear (left) and $\log$ (right) scale in $t \bar{t} \rightarrow e \mu$, background and data events having one good electron and one good muon with $\Delta R(e, \mu)>0.25 \ldots \ldots \ldots \ldots \ldots \ldots \ldots \ldots \ldots \ldots \ldots \ldots \ldots$

5.20 Distributions of the detector $\eta$ (left) and $p_{T}$ (right) of electrons in $t \bar{t} \rightarrow e \mu$, background and data events having one good electron and one good muon with $\Delta R(e, \mu)>0.25$, and $\geq 1$ jet with $p_{T}>20 \mathrm{GeV} . \ldots 256$

5.21 Distribution of $p_{T}$ of the leading lepton in $t \bar{t} \rightarrow e \mu$, background and data events having one good electron and one good muon with $\Delta R(e, \mu)>0.25$,

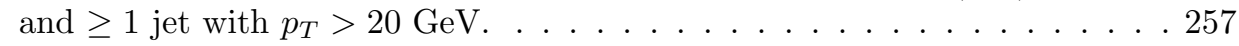

5.22 Distributions of $\eta$ (left), $p_{T}$ (right) of muons in $t \bar{t} \rightarrow e \mu$, background and data events having one good electron and one good muon with $\Delta R(e, \mu)>$ 0.25 , and $\geq 1$ jet with $p_{T}>20 \mathrm{GeV} \ldots \ldots \ldots \ldots \ldots \ldots$ 
5.23 Distributions of $\eta$ (left), $p_{T}$ (right) of the leading jet in $t \bar{t} \rightarrow e \mu$, background and data events having one good electron and one good muon with $\Delta R(e, \mu)>0.25$, and $\geq 1$ jet with $p_{T}>20 \mathrm{GeV} \ldots \ldots \ldots \ldots 258$

5.24 Distributions of the $E_{T}$ (left), $H_{T}^{\text {leading lepton }}$ (right) in $t \bar{t} \rightarrow e \mu$, background and data events having one good electron and one good muon with $\Delta R(e, \mu)>0.25$, and $\geq 1$ jet with $p_{T}>20 \mathrm{GeV} \ldots \ldots \ldots \ldots 258$

5.25 Distributions of detector $\eta$ (left) and $p_{T}$ (right) of electrons in $t \bar{t} \rightarrow e \mu$, background and data events after applying all selection criteria (see Tab. 5.1).259

5.26 Distributions of $\eta$ (left) and $p_{T}$ (right) of muons in $t \bar{t} \rightarrow e \mu$, background and data events after applying all selection criteria (see Tab. 5.1). . . . . . 259

5.27 Distributions of $p_{T}$ (left) and $\eta$ (right) of the leading jet in $t \bar{t} \rightarrow e \mu$, background and data events after applying all selection criteria (see Tab. 5.1). . 260

5.28 Distribution of (a) $p_{T}$ of the leading lepton and (b) $H_{T}^{j e t}$ in $t \bar{t} \rightarrow e \mu$, background and data events after applying all selection criteria (see Tab. 5.1). . 260

5.29 Distributions of $E_{T}$ (left), $H_{T}^{\text {leading lepton }}$ (right) in $t \bar{t} \rightarrow e \mu$, background and data events after applying all selection criteria (see Tab. 5.1). . . . . . . 261

5.30 Distributions of number of jets with $p_{T}>20 \mathrm{GeV}$ in $t \bar{t} \rightarrow e \mu$, background and data events after applying all selection criteria (see Tab. 5.1). . . . . . 261

5.31 The $t \bar{t} \rightarrow e \mu$ event selection efficiency (in arbitrary units) as function of the mass of the top quark. The present analysis is based on a MC sample with top quark mass of $175 \mathrm{GeV} / \mathrm{c}^{2}$ (shown in red marker). . . . . . . . . . . . 269

5.32 Likelihood as a function of the $t \bar{t}$ production cross section. The central value and the statistical errors are indicated as vertical line. . . . . . . . . 275

5.33 Results of a toy Monte Carlo experiment with $5.14 \pm 0.58$ signal (with a $t \bar{t}$ production cross section of $7 \mathrm{pb}$ ) and $0.91 \pm 0.20$ background events. The observed number of events i.e., 8 is shown with the arrow. . . . . . . . 278

A.1 Schematic view of a $Z^{0} \rightarrow \tau \tau \rightarrow e \mu+$ jets event. . . . . . . . . . 286

C.1 $e-\mu$ candidate event: Run 179141 Event 11709332; RZ view (upper right), XY view (upper left), Lego view (lower). . . . . . . . . . . . . . . 298

C.2 $e-\mu$ candidate event: Run 178733 Event 8735139; RZ view (upper right), XY view (upper left), Lego view (lower). . . . . . . . . . . . . . . . . 299

C.3 $e-\mu$ candidate event: Run 179195 Event 26386170; RZ view (upper right), XY view (upper left), Lego view (lower). . . . . . . . . . . . . . . . 300

C.4 $e-\mu$ candidate event: Run 179331 Event 19617819; RZ view (upper right), XY view (upper left), Lego view (lower). . . . . . . . . . . . . . . . . 301

C.5 $e-\mu$ candidate event: Run 177826 Event 15259654; RZ view (upper right), XY view (upper left), Lego view (lower). . . . . . . . . . . . . . . . . 302

C.6 $e-\mu$ candidate event: Run 178159 Event 37315438; RZ view (upper right), XY view (upper left), Lego view (lower). . . . . . . . . . . . . . . . . 303

C.7 $e-\mu$ candidate event: Run 168393 Event 1997007 ; RZ view (upper right), XY view (upper left), Lego view (lower). . . . . . . . . . . . . . . . . 304

C.8 $e-\mu$ candidate event: Run 174901 Event 8710859 ; RZ view (upper right), XY view (upper left), Lego view (lower) . . . . . . . . . . . . . . . 305 


\section{List of Tables}

1.1 The fundamental particles of the Standard Model . . . . . . . . . . . . . . . 9

1.2 Theoretical cross sections, at next-to-leading-order in QCD, for top-quark production via the strong interaction at the Tevatron and the LHC [26]. Also shown is the percentage of the total cross section from the quarkantiquark-annihilation and gluon-fusion subprocesses. . . . . . . . . . 26

1.3 Decay modes of $W^{ \pm}$and its branching fractions [32] . . . . . . . . . . 31

2.1 Geometrical parameters of different SMT components. . . . . . . . . . . . . 64

2.2 Physical parameters of CFT layers. U and V layers are at stereo angles of

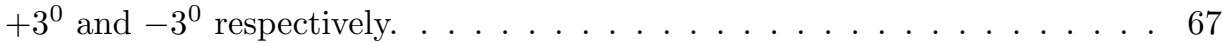

2.3 Central Calorimeter Parameters. . . . . . . . . . . . . . . . . . . . . 80

2.4 End Calorimeter Parameters. . . . . . . . . . . . . . . . . . . . . . 82

4.1 Summary of the integrated luminosity for the data sample. . . . . . . . . . 164

4.2 Triggers used in the electron-muon analysis; note there is no requirement at Level 2 of Trigger system. . . . . . . . . . . . . . . . . . 166

4.3 Parameters of the Trigger Turn-on curves used in the electron-muon analysis. 175

4.4 Trigger efficiencies for different Monte Carlo processes. Errors are statistical.176

4.5 Values for scale factor $(\alpha)$ and smearing parameter (c) of Eqn. 4.16 for different types of electrons. . . . . . . . . . . . . . . . . . . . 189

4.6 Jet resolution constants for $\mathrm{MC}$ and data. . . . . . . . . . . . . . 195

5.1 Event selection criteria for rejecting different background processes. . . . . . 205

5.2 Primary vertex cuts efficiencies (in \%) in $Z^{0} / \gamma^{*} \rightarrow e e$ and $Z^{0} / \gamma^{*} \rightarrow \mu \mu$ for data and MC and a scale factor as a function of jet multiplicity. All uncertainties are statistical. . . . . . . . . . . . . . . . . . . . 212

5.3 Scale factors related to primary vertices derived from Tab. 5.2. Statistical errors are taken as the uncorrelated sum of the statistical errors from the $Z^{0} / \gamma^{*} \rightarrow e e$ and $Z^{0} / \gamma^{*} \rightarrow \mu \mu$ samples. . . . . . . . . . . . . . . 213

5.4 Data-to-MC scale factors for the medium electron efficiency, the track match times likelihood efficiency and for the track match efficiency. The systematic uncertainties associated to each scale factor are also given. . . . . . . . . . 216 
5.5 Per event efficiencies in $Z^{0} / \gamma^{*} \rightarrow \mu \mu$ data and Monte Carlo for the $D C A$ cut and the corresponding scale factors. The quoted errors are statistical only. These efficiencies are derived for muons with a transverse momentum of at least $15 \mathrm{GeV}$. . . . . . . . . . . . . . . . . . . . . . 222

5.6 Per muon efficiencies in $Z^{0} / \gamma^{*} \rightarrow \mu \mu$ data and Monte Carlo samples for the muon isolation cut and the corresponding scale factor. The isolation efficiency with the additional requirement $\Delta R(\mu, j e t)>0.5$ is provided as a cross check. The quoted errors are statistical only. . . . . . . . . . . 225

5.7 Measured efficiencies per dimuon event for the $\chi^{2}<4$ cut (on the muon track) in $Z^{0} / \gamma^{*} \rightarrow \mu \mu$ data and Monte Carlo samples, for different jet multiplicities. . . . . . . . . . . . . . . . . . . 226

5.8 Efficiencies of different selection cuts on a $t \bar{t} \rightarrow e \mu$ Monte Carlo sample and the data to Monte Carlo scale factors $(\kappa)$. The scale factors are explained in Sec. 5.3. Errors on the scale factors are not quoted here, as they are treated as systematic errors and are listed in Sec. 5.7. . . . . . . . . . . . 229

5.9 The rate for photon faking an electron candidate, $f_{\gamma}$, is estimated from Monte Carlo samples, as a probability of isolated photon cluster to pass track match and likelihood criteria. The rate at which photon clusters pass track match criteria is also provided separately. The average is taken over the rates from two samples (viz., $W^{ \pm} \gamma \rightarrow \mu \nu_{\mu} \gamma$ and $Z^{0} \gamma \rightarrow \mu \mu \gamma$ ). . . . . 235

5.10 Expected event yields for various photon background processes. All errors are statistical. . . . . . . . . . . . . . . . . . . . 237

5.11 Estimated $f_{e}$ and event yield for fake electrons corresponding to an event selection criteria of having one tight electron and one tight muon with $\Delta R(e, \mu)>0.25$ (see Tab. 5.1) in the event. . . . . . . . . . . . . 241

5.12 Various EM and muon definitions used in Matrix method. . . . . . . . . . . 243

5.13 Signal and fake efficiencies for electrons and muons applied in four-component Matrix method. . . . . . . . . . . . . . . . . . . . . 245

5.14 Events observed for different loose and tight configurations for electrons and muons with $\Delta R(e, \mu)>0.25 \ldots \ldots \ldots \ldots \ldots \ldots \ldots$

5.15 Number of events determined in loose-loose and tight-tight data samples

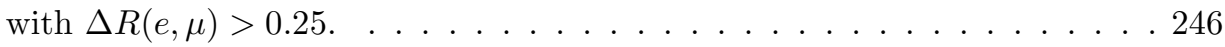

5.16 Comparison between different methods for photon and fake background estimation. The events required to have one electron and one muon with $\Delta R(e, \mu)>0.25$. The errors are purely statistical. . . . . . . . . . 250

5.17 Data, expected signal and backgrounds at each level of selection in electronmuon channel, with statistical errors added quadratically to the systematic uncertainties. The expected signal yield is based on a $t \bar{t}$ production cross

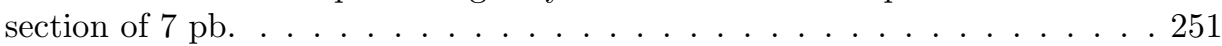

5.18 Expected background and observed and expected signal yields. The expected signal yield assumes a $7 \mathrm{pb} t \bar{t}$ production cross section. . . . . . . . . 252

5.19 Run numbers and event numbers for the $e \mu$ candidate events. . . . . . . . . 262 
5.20 Summary of the relative systematic uncertainties (in \%) on signal and different background processes after applying all selection criteria mentioned in Tab. 5.1. The estimate for instrumental fake events has a $39 \%$ uncertainty on its value which is not quoted here, as the instrumental fake events of this analysis are directly estimated from data. . . . . . . . . . . . . . 272

5.21 Values of the variables which are utilised to estimate the $t \bar{t}$ production cross section in $e \mu$ channel. . . . . . . . . . . . . . . . . . . . 273

C.1 Kinematics for Event Number:11709332 Run Number:179141 . . . . . . . . 293

C.2 Kinematics for Event Number:8735139 Run Number:178733 . . . . . . . . . 294

C.3 Kinematics for Event Number:26386170 Run Number:179195 _. . . . . . . . 294

C.4 Kinematics for Event Number: 19617819 Run Number: 179331 . . . . . . . 294

C.5 Kinematics for Event Number: 15259654 Run Number: 177826 . . . . . . . . 295

C.6 Kinematics for Event Number: 37315438 Run Number:178159 . . . . . . . . . 295

C.7 Kinematics for Event Number: 1997007 Run Number: 168393 . . . . . . . . . 295

C.8 Kinematics for Event Number: 8710859 Run Number: 174901 . . . . . . . . 296 


\section{Chapter 1}

\section{Introduction}

Quest for the ultimate building block of matter has been pursued by the mankind for many millennia. Particle physics is concerned with these fundamental units of matte and the nature of interactions between them. This chapter briefly narrates the road to the present state of particle physics, starting from the ancient days of science. The Standard Model of elementary particle physics has been briefly summarised. The production and decay of top quark has been described. 


\subsection{History}

Ancient Indians used to believe that the universe is made up of space, earth, air, fire and water. In a similar spirit according to ancient Greek philosophy, the basic elements were air, fire, water and earth. The concept of an "atom"like structure of matter was introduced by the Greek philosophers (Leucippos and Democritus) in 400 B.C. And finally in 1870, Russian chemist Dmitri Mendeleev was successful in arranging the elements into a periodic table by looking at the repetitive pattern in chemical properties of the elements. By rearranging the elements in this specific way many physical and chemical properties could be predicted with precision but it was too complicated to represent the basic/fundamental building blocks of matter. The proliferation of elements and apparent systematics in the organization of Mendeleev's table strongly favoured a substructure.

Towards the end of nineteenth century scientists started believing that the elements do have some substructure. In 1897, while studying the deflection of cathode rays under the influence of magnetic field, J. J. Thomson discovered negatively charged particles, called electrons [1]. Thomson correctly inferred that these electrons are essential constituents of atoms. However, since the electrically neutral atoms are much heavier than electrons, Thom- 
son hypothesized the "plum pudding model" of atom where, the electrons are suspended in a heavy, positively charged paste, like plums in a pudding. Thomson's model was repudiated by Ernest Rutherford's scattering experiment [2] in 1911, at Cavendish Laboratory (England). Bombardment of $\alpha$ particles (doubly ionized helium atoms) on a thin gold foil revealed the fact that, the atom contains a positively charged central part (nucleus) where the mass of the atom is concentrated. The nucleus of a hydrogen atom, the lightest element, was given the name "proton" by Rutherford.

In 1914, Danish physicist Niels Bohr suggested a mathematical description of the atomic structure based on Max Planck's theory of energy quantization. Bohr proposed a model [3] for the hydrogen atom, where the electron rotates around the proton in certain fixed orbits through Coulomb attraction of opposite charges. With help of primitive version of quantum theory, Bohr successfully calculated the spectra of hydrogen energy levels which had spectacular agreement with experimental results. It was natural then to consider the nuclei of heavier atoms to be composed of two or more protons. However, the next heavier atom (helium) although has two electrons, weighs four times as much as hydrogen, and lithium (three electrons) is seven times heavier than hydrogen and so on. This mystery was solved in 1932, when James Chadwick proved the existence of "neutron" [4], an electrically neu- 
tral particle and of approximately the same mass as proton. It turned out that the helium nucleus contains two neutrons in addition to two protons; lithium includes four and so on. Till 1930's, the set of elementary particles consisted of proton, neutron and electron. However observed phenomena like nuclear $\beta$-decay and binding forces between protons and neutrons in a atomic nucleus, were mysteries to the scientific community.

With the advent of relativistic quantum mechanics by Paul Dirac $[5]^{\ddagger}$ the existence of "positron" (a positively charged particle of the same mass as electron) was postulated in 1928 as the anti-particle of electron and was established experimentally in 1933. Italian physicist, Enrico Fermi solved the puzzle of radioactive $\beta$-decay [7] by predicting a mass-less particle called "neutrino" (first postulated by Pauli in 1930) along with a theory of "weak interactions". The electron neutrinos were experimentally detected much later in 1953 by Frederick Reines and Clyde Cowan [8].

In the framework of "classical" model of atom, the scientific community still had one conspicuous problem: What holds the protons and neutrons together in an atomic nucleus? In 1935, Hideki Yukawa utilized the concept of "isospin" introduced by Heisenberg, to postulate a new kind of interaction

\footnotetext{
${ }^{\ddagger}$ Later in 1949, Feynman greatly generalised Dirac’s theory into a "propagator" formalism which was named Quantum Electrodynamics (QED) [6].
} 
("strong interaction") which can be represented as an exchange of massive quanta. This provided a radical explanation to the nuclear binding forces between protons and neutrons in a nucleus [9]. Three massive particles, called "pions" (experimentally found to have masses very close to Yukawa's prediction, in 1947) were added to the list of elementary particles. While trying to detect the pions (as predicted by Yukawa's theory) in cosmic rays, Powell and his co-workers at Bristol [10] discovered a new particle, which was different from Yukawa's prediction. The newly discovered particle was called "muon" and was found to have a mass of 206 times the electron mass.

By the middle of twentieth century, the then known elementary particles were insufficient to explain phenomena observed in nuclear physics experiments and cosmic ray studies. Moreover, many new particle states were found in experiments based on particle accelerators. These states are grouped into a class of particles called "hadrons" which is subdivided into heavier "baryons" and lighter "mesons". Neutron, proton and pions turned out to be the lightest members of these groups, respectively. In 1964, Gell-Mann [11] and George Zewig incorporated these particles into a SU(3) symmetry group where the hadrons are made of three more fundamental constituents called "quarks" - named up (u), down (d) and strange (s). Hadrons are believed to be bound states of quarks which exchange quanta of strong interactions 
called "gluons" among themselves. The model was supported by the results from Stanford Linear Accelerator (in 1970) where neutron and proton were proved to have internal structure.

In 1974, a SLAC experiment discovered the existence of another new particle called "tau" $(\tau)$. Electron $(\mathrm{e})$, muon $(\mu)$ and tau $(\tau)$ along with the corresponding neutrinos $\left(\nu_{e}, \nu_{\mu}\right.$ and $\left.\nu_{\tau}^{\ddagger}\right)$ are grouped into a class of elementary particles called "leptons". The 1960's model of weak interactions, represented by a four-fermion theory, ran into the serious problem of "renormalizability" when applied to higher-order calculations. Attempts to describe weak interactions in terms of heavy boson exchange were successful when the unified theory of electromagnetic and weak interactions, proposed by Weinberg, Glashow and Salam [13] predicted three massive vector bosons $\left(W^{+}, W^{-}\right.$and $\left.Z^{0}\right)$ as the mediators of electroweak interactions. The triumph of electroweak unification followed in 1983 when CERN Spp̄S collider experiments UA1 and UA2 discovered $W^{+}, W^{-}$and $Z^{0}$ particles [14].

After the successful proposition and experimental evidence for the quark model in 1964, three more quarks have been discovered experimentally charm (c) in 1974 at SLAC and BNL, bottom (b) in 1977 and top (t) in 1995

\footnotetext{
${ }^{\ddagger} \nu_{\tau}$ has been observed in 2002 by the DONUT collaboration [12] while, the muon neutrino was detected experimentally at Brookhaven National Laboratory by Leon Lederman, Melvin Schwartz and Jack Steinberger in 1962.
} 
at Fermilab. Detailed theoretical calculations by Gell-Mann, 't Hooft, Gross, Wilczek and Politzer led to the formulation of "Quantum Chromodynamics" (QCD) as a non-Abelian gauge theory of strong interactions. Theories of electroweak unification and QCD along with experimental observations, have led to the present state of affairs, called "The Standard Model" of elementary particles.

\subsection{The Standard Model}

The universe appears to be governed by four kinds of fundamental forces - strong, electromagnetic, weak and gravitational. They are of markedly different strengths as shown in Fig. 1.1. The Standard Model (SM) of elementary particles encompasses the progress that has been made in past century in understanding the weak, electromagnetic and strong interactions. Gravitational force is by far the weakest and is important for large bodies. It has no influence at the subatomic level $\left(\approx 10^{-15}\right.$ metre $)$, dealt with SM.

The SM contains both fermionic and bosonic fundamental particles and they are classified into three groups: quarks, leptons and gauge bosons. Quarks and leptons are all spin- $\frac{1}{2}$ particles and thus obey Pauli exclusion principle. There are six quark flavours which are divided into three gen- 


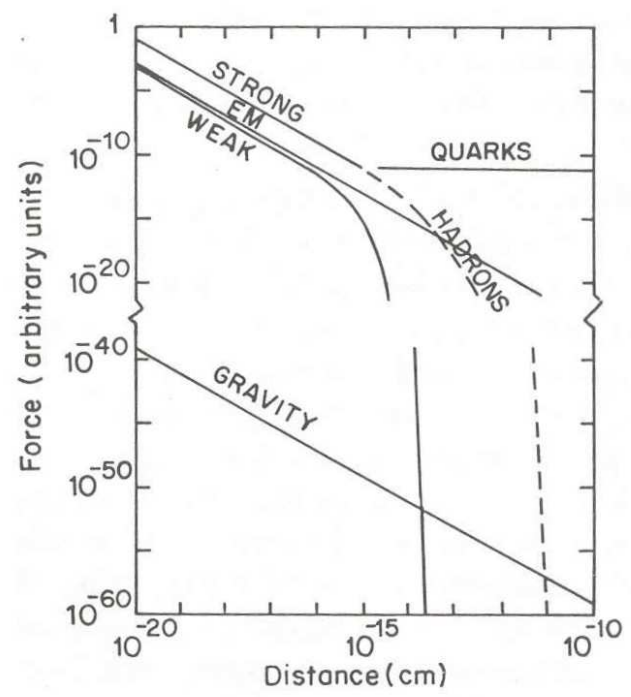

Figure 1.1: Four types of fundamental forces between a typical pair of particles.

erations (also called families) each consisting of a doublet: $(\mathrm{u}, \mathrm{d}),(\mathrm{c}, \mathrm{s})$ and (t,b). Similar classification is also valid for leptons: $\left(e, \nu_{e}\right),\left(\mu, \nu_{\mu}\right)$ and $\left(\tau, \nu_{\tau}\right)$. Tab. 1.1 summarizes the properties of the fundamental particles in SM. All normal matter are composed of the particles from the first generation. In high energy collisions, particles of higher generations are created but they appear to be unstable; all higher generation particles ultimately, decay into the first generation particles or photons. This is pictorially depicted in Fig. 1.2

Each generation of leptons consists of one charged particle having one unit charge (electron, muon or tau) and an associated uncharged particle 


\begin{tabular}{|c|c|c|c|c|}
\hline \multirow{7}{*}{$\begin{array}{r}\text { Quarks } \\
(\operatorname{spin}=1 / 2)\end{array}$} & symbol & name & $\operatorname{mass}\left(\mathrm{MeV} / \mathrm{c}^{2}\right)$ & charge $(e)$ \\
\hline & $d$ & down & $\approx 8$ & $-1 / 3$ \\
\hline & $u$ & up & $\approx 4$ & $2 / 3$ \\
\hline & $s$ & strange & $\approx 150$ & $-1 / 3$ \\
\hline & $c$ & charm & $\approx 1400$ & $2 / 3$ \\
\hline & $b$ & bottom & $\approx 4500$ & $-1 / 3$ \\
\hline & $t$ & top & $\approx 175 \mathrm{GeV} / \mathrm{c}^{2}$ & $2 / 3$ \\
\hline \multirow{6}{*}{$\begin{array}{r}\text { Leptons } \\
(\operatorname{spin}=1 / 2)\end{array}$} & $e$ & electron & 0.511 & -1 \\
\hline & $\nu_{e}$ & electron neutrino & $<3 \mathrm{eV} / \mathrm{c}^{2}$ & 0 \\
\hline & $\mu$ & muon & 105.7 & -1 \\
\hline & $\nu_{\mu}$ & muon neutrino & $<0.19$ & 0 \\
\hline & $\tau$ & tau & 1777 & -1 \\
\hline & $\nu_{\tau}$ & tau neutrino & $<18.2$ & 0 \\
\hline \multirow{4}{*}{$\begin{array}{l}\text { Gauge bosons } \\
\quad(\operatorname{spin}=1)\end{array}$} & $\gamma$ & photon & 0 & 0 \\
\hline & $W$ & $W$ & $80.4 \mathrm{GeV} / \mathrm{c}^{2}$ & 1 \\
\hline & $Z$ & $Z$ & $91.2 \mathrm{GeV} / \mathrm{c}^{2}$ & 0 \\
\hline & $g$ & gluon & 0 & 0 \\
\hline Higgs & $H^{a}$ & Higgs & $?$ & 0 \\
\hline
\end{tabular}

Table 1.1: The fundamental particles of the Standard Model

${ }^{a}$ Not yet observed. 


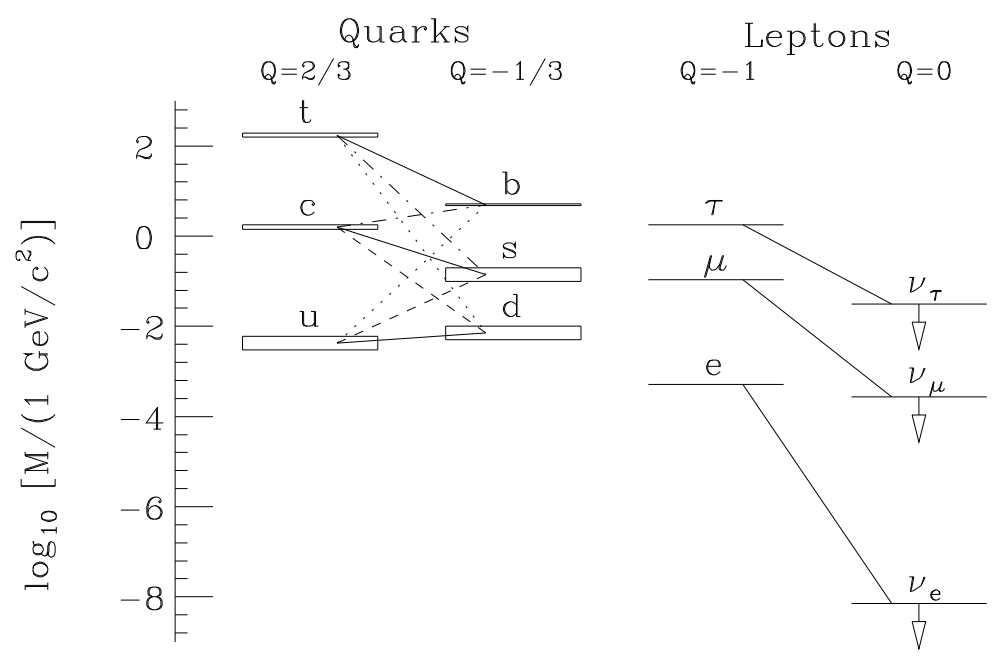

Figure 1.2: Patterns of charge-changing weak transitions among quarks and leptons. The strongest inter-quark transitions correspond to the solid lines, with dashed, dot-dashed, and dotted lines corresponding to successively weaker transitions.

(neutrinos). The SM treats the neutrinos as the massless particles while experimentally their masses are constrained to be very low. The charged leptons take part in electromagnetic and weak interactions, but the neutrinos are only affected by weak interactions. Quarks on the other hand are of fractional electric charges (either $1 / 3$ or $2 / 3$ the charge of an electron) and take part in strong interactions.

In the framework of SM, the gauge bosons are responsible for the interactions between the particles. Photon for example, is the mediator of electromagnetic interaction and couples to all fermions except the neutrinos. 
Similarly, Weak interactions are mediated by $W^{ \pm}$and $Z^{0}$ bosons and operate only at short distances $\left(\mathcal{O}\left(10^{-17}\right.\right.$ metre $)$, as shown in Fig. 1.1. In SM, the electromagnetic force and weak force are treated in a unified manner (electroweak theory). The parity violating weak interaction is described by the $\mathrm{SU}(2)_{\mathrm{L}}$ gauge symmetry acting on the left-handed fermions while the electromagnetic interaction is well-described by the $\mathrm{U}(1)$ symmetry. Before spontaneous symmetry breaking through Higgs mechanism, all the gauge bosons are massless. When the symmetry is spontaneously broken the gauge particles involved in charged $\left(W^{ \pm}\right)$and neutral $\left(Z^{0}\right)$ current exchange acquire masses.

To be consistent with the Pauli exclusion principle, the quarks are required to have an additional quantum number, called "colour", apart from the flavour quantum number $(\mathrm{S}, \mathrm{C}, \mathrm{B}$ and $\mathrm{T})$. The strong interaction takes place between such "colour"-ed objects and is mediated by gluons. A quark of specific flavour comes in three colours - conventionally named as "red (R)", "green $(\mathrm{G})$ " and "blue (B)" (anti-quarks come in "anti-red $(\overline{\mathrm{R}})$ ", "anti-green $(\overline{\mathrm{G}})$ " and "anti-blue $(\overline{\mathrm{B}})$ ") while gluons are of 8 possible colour charges: $\mathrm{R} \overline{\mathrm{G}}$, $\mathrm{R} \overline{\mathrm{B}}, \mathrm{G} \overline{\mathrm{R}}, \mathrm{G} \overline{\mathrm{B}}, \mathrm{B} \overline{\mathrm{R}}, \mathrm{B} \overline{\mathrm{G}}, \sqrt{\frac{1}{2}}(\mathrm{R} \overline{\mathrm{R}}-\mathrm{G} \overline{\mathrm{G}}), \sqrt{\frac{1}{6}}(\mathrm{R} \overline{\mathrm{R}}+\mathrm{G} \overline{\mathrm{G}}-2 \mathrm{~B} \overline{\mathrm{B}})$. Since the gluons carry two labels, one colour and one anticolour, colour is conserved at each quark-quark-gluon vertex. For example, a blue quark can turn into a 
red quark by emitting a blue-anti-red gluon. The hadrons are colour-singlet combinations of quarks, anti-quarks and gluons. In contrast to the electromagnetic interaction, the strength of the strong interaction decreases with the increase in energy of interaction. As a consequence, quarks behave like free particles ("asymptotic freedom") at the energy of the present generation high energy physics experiments $(E \gtrsim 10 \mathrm{GeV})$. In this region, the behaviour of the strong force can be calculated using perturbation techniques. However, at low energies (bound states of quarks in a nucleon) where the coupling strength becomes large enough, the behaviour of strong force is not calculable using the perturbation theory as it breaks down at those energy scales. The variation of strong coupling constant $\left(\alpha_{s}\right)$ with interaction energy is shown in Fig 1.3.

Since the magnitude of strong force increases with the increase in the distance between the quarks, neither quarks nor gluons can appear in isolation, they can only exist within colourless (colour-neutral) composite hadrons. In a bound state, the colour charges of constituent quarks cancel one another (either a quark and its antiquark with opposite colour, or a mixture of all three colours) and produce a colour-less state. To separate one quark out of a bound state, sufficient amount of energy is required first to produce a new quark-antiquark pair. One of the created quarks forms a pair 


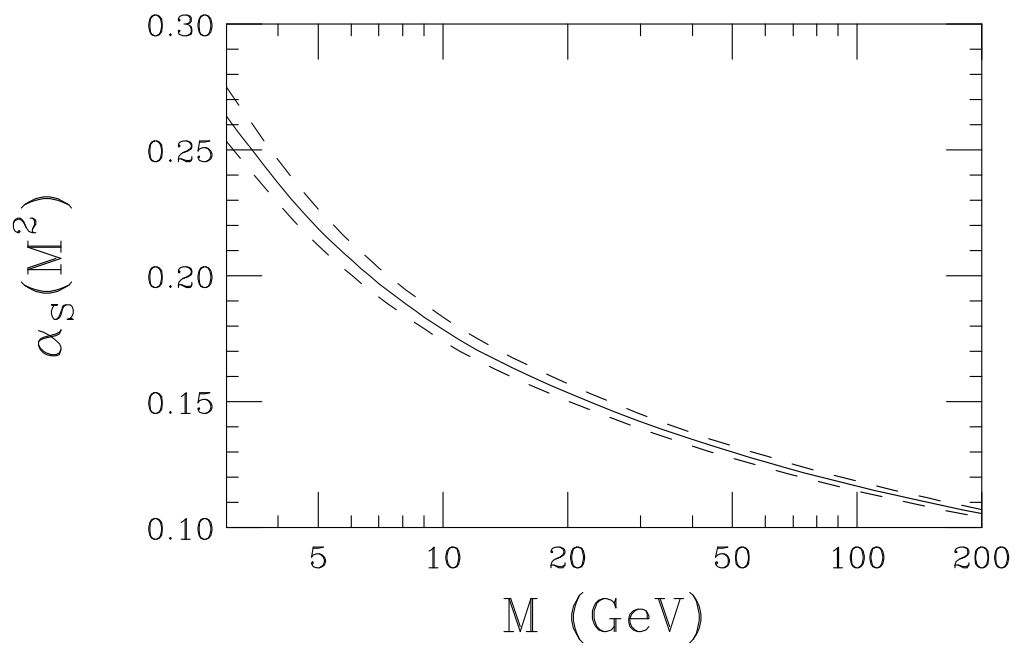

Figure 1.3: The strong-coupling constant $\alpha_{S}\left(M^{2}\right)$ as a function of energy scale $(\mathrm{M})$, subject to the constraint $\alpha_{S}\left(M_{Z}^{2}\right)=0.118 \pm 0.002$. The solid line shows the central value; dashed lines indicate $\pm 1 \sigma$ limits.

with the quark that was removed from the bound state, while the other one takes the place of the removed quark, as shown in Fig 1.4. This process is called "fragmentation". Experimentally fragmentation is seen as a collimated set of hadrons (called "jet") in a direction close to the original hadron direction.

The Standard Model posits the existence of one complex weak doublet of elementary scalar (Higgs) field in order to provide a mechanism for giving masses to the otherwise massless gauge particles. Higgs boson interacts with the weak charge carriers $\left(W^{ \pm}\right.$and $\left.Z^{0}\right)$ in exactly the right way so that they acquire masses. Through this mechanism, quarks and leptons can also 


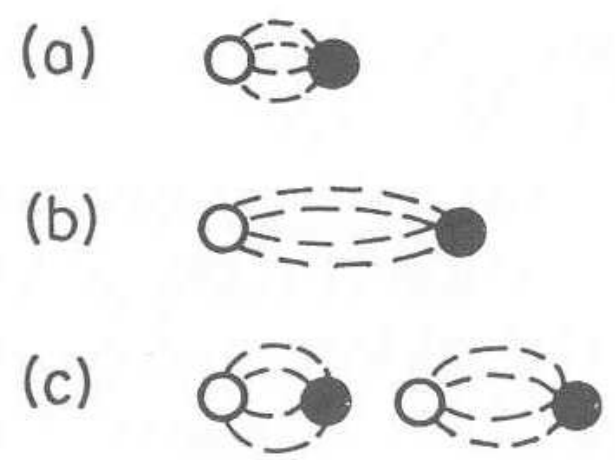

Figure 1.4: Typical effect of injecting energy into a hadron to separate the quarks: (a) original quark -antiquark pair held by colour lines of force; (b) lines of force are stretched; (c) lines of force break with the creation of a new quark-antiquark pair.

acquire masses. This real observable particle has to be observed experimentally, in order to validate the SM fully. Till today, Higgs boson has not been observed in experimental searches. In SM, the mass of top quark and the mass of $\mathrm{W}$ together allow us to constraint the mass of the Higgs boson. The latest experimental measurements related to $\mathrm{W}$ mass and top mass are shown in Fig. 1.8. A further detailed description of the latest status of SM can be found in $[15,16]$. 


\subsection{Top Quark}

In SM, top quark is a spin- $\frac{1}{2}$ third generation fermion of electric charge $+\frac{2}{3}$ and transforms as a colour anti-triplet under $\mathrm{SU}(3)$ gauge group of strong interactions. The tau lepton $(\tau)$ was the first particle from the third generation of particles to be discovered [17]. Shortly after-wards in 1977, $\Upsilon$ was discovered at Fermilab as a resonance in the $\mu^{+} \mu^{-}$invariant mass spectrum [18] and was interpreted as a bound state of $b \bar{b}$ which decays into $\mu^{+} \mu^{-}$. At this point top quark was the only quark which was not observed experimentally. But there were several compelling reasons to believe that the top quark must exist.

Finally in 1995, top quark was discovered at the Fermilab's Tevatron collider jointly by both CDF and D $\varnothing$ collaborations $[19,20]$ which confirmed the three-generation structure of the SM. At Tevatron it is observered that the top quarks are produced only in pairs (top and anti-top). The production cross-sections have been measured at a centre of mass energy of $1.8 \mathrm{TeV}$ (Tevatron Run I) by both the experiments and agree quite well with theoretical calculations (Fig. 1.5). Subsequently, mass of the top quark has been measured by both experiments in different decay channels yielding a world average top mass of $178.0 \pm 4.3 \mathrm{GeV} / \mathrm{c}^{2}$ (see Fig. 1.6) and is the best measured 
among all quark masses, with a fractional error of $2.4 \%$. Due to the rarity of top events in Tevatron Run I, measurements of many other properties of top quark either suffer from large statistical uncertainties or remain beyond the scope of experimental exploration. Few such properties are discussed in the next section on which, statistically significant measurements are expected during Run II of Tevatron and LHC.

\subsubsection{Important Aspects of Top Quark}

The most striking feature of top quark is its unusually high mass which sets it apart from other quarks. It is almost 40 times heavier than the next heaviest quark, bottom quark, and is the heaviest known elementary particle till the date. Since its mass is close to the electro-weak scale, the top quark could play an important role in the electro-weak symmetry breaking mechanism of SM. The Yukawa coupling $\left(Y_{t}\right)$ of top quark which relates the top quark with the Higgs field, fundamentally depends on the top quark mass viz., $\mathrm{Y}_{\mathrm{t}}=\frac{\sqrt{2} \cdot \mathrm{m}_{\mathrm{t}}}{v}$, where $\mathrm{m}_{\mathrm{t}}$ and $v$ are the top mass and the Higgs vacuum expectation value respectively. With $v=246 \mathrm{GeV}$ and $\mathrm{m}_{\mathrm{t}}=178.0 \pm 4.8$ $\mathrm{GeV}, \mathrm{Y}_{\mathrm{t}}=1.02 \pm 0.03$, which leads to the theoretical speculation that new physics could be accessible through studies of top quark. Moreover, as both 


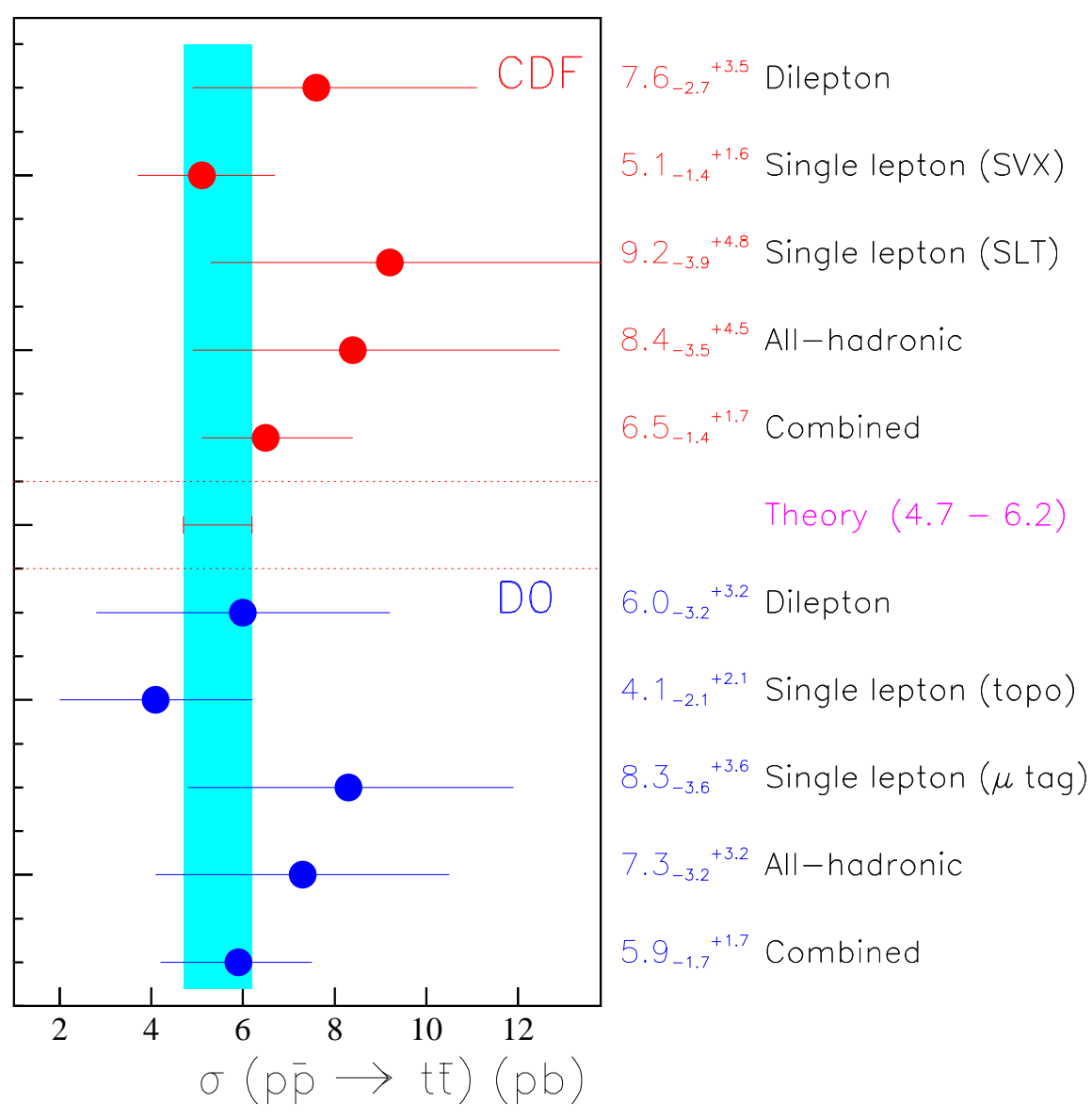

Figure 1.5: $\mathrm{CDF}$ and $\mathrm{D} \varnothing$ cross section results for $t \bar{t}$ production at the Fermilab Tevatron, Run I (with $\sqrt{s}=1.8 \mathrm{TeV}$ ), overlaid with the theory prediction. For the latter, the entire band includes the NLO+NLL and partial NNLO+NNLL predictions. 


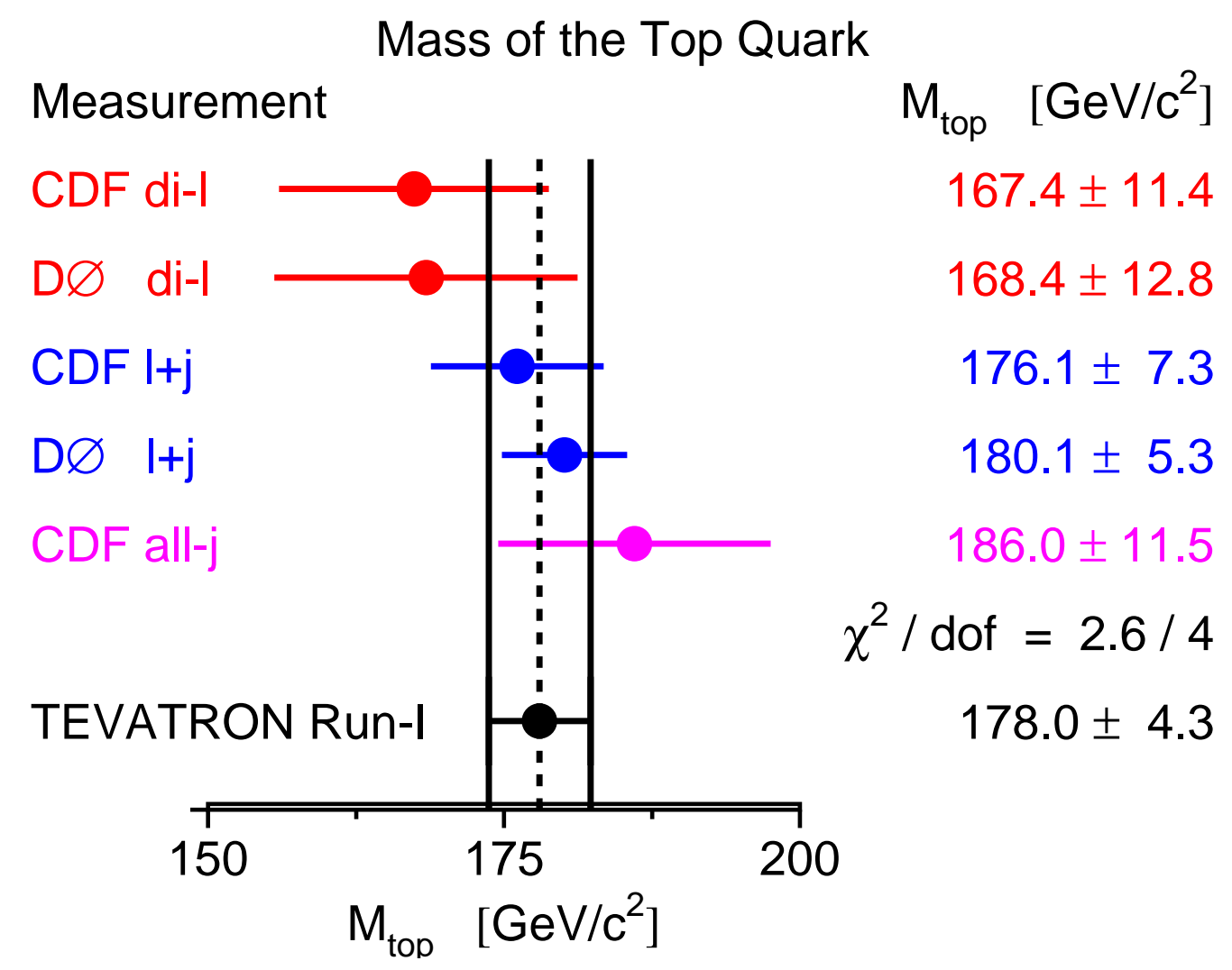

Figure 1.6: Comparison of CDF and D $\varnothing$ measurements of the top quark mass at the Fermilab Tevatron, Run I (with $\sqrt{s}=1.8 \mathrm{TeV}$ ) [21]. 
top quark and Higgs boson contribute to the electroweak radiative correction to $\mathrm{W}$ boson mass (Fig. 1.7), precise measurement of top quark mass along with $\mathrm{W}$ boson mass would constrain the Higgs mass (Fig. 1.8). Thus a precise measurement of top quark mass is extremely crucial for a detailed scrutiny of SM and also for Higgs search.

Top pair-production cross-sections as described in Sec. 1.3.2, can be calculated using perturbative QCD with a well defined scale. Any statistically significant excess in the measured cross section would indicate new physics beyond the SM. On the other hand, in the SM framework top quark is expected to decay mostly into a bottom quark and a $\mathrm{W}$ boson since $\left|\mathrm{V}_{\mathrm{tb}}\right| \approx 1$. Thus, accurate measurements of other decay modes of top quark as detailed in Sec. 1.3.3, e.g., the ratio of branching fractions $\mathcal{R}=\mathcal{B}(\mathrm{t} \rightarrow \mathrm{Wb}) / \mathcal{B}(\mathrm{t} \rightarrow \mathrm{Wq})$ $=\frac{\left|\mathrm{V}_{\mathrm{tb}}\right|^{2}}{\left|\mathrm{~V}_{\mathrm{td}}\right|^{2}+\left|\mathrm{V}_{\mathrm{ts}}\right|^{2}+\left|\mathrm{V}_{\mathrm{tb}}\right|^{2}}$, would be a good test for SM predictions. CDF reported a measurement of $\mathcal{R}[22]$ based on Run I Tevatron data, while D $\varnothing$ has performed a measurement of the same [23] with better accuracy during Tevatron Run II.

One of the notable features of the top quark is that it is the only quark whose spin is a directly observable quantity. This is a consequence of its very short lifetime, which is shorter than the typical time scale of hadronisation. As a result, the top quark leaves an imprint of its spin information on its 
angular decay distribution. Therefore, a direct measurement of the angular correlation between the decay products of top and anti-top quarks, would correlate the spins of top and antitop quarks. DØ has already reported this measurement [24] using Run I data of Tevatron. Greater precision on this measurement is expected during Run II.

Lastly, top quark production at the hadron collider is an ideal place to look for physics beyond SM. For example, the Minimal Supersymmetric extension of SM called MSSM, assigns a bosonic (fermionic) superpartner to every fermion (boson) in the SM and if the superpartner of the top quark ('stop') exists, it must show up in the top events. In such a scenario, one

should observe an excess in the measured $t \bar{t}$ production cross section over the SM prediction. Besides MSSM, there are many theories related to new physics where events containing top quark can appear as a potential background process. Thus, in order to probe these models, study of top quark is of fundamental interest.

\subsubsection{Production of Top Quark at Tevatron}

At the current centre of mass energy of $1.96 \mathrm{TeV}$ available at Tevatron and within the Standard Model, the top quarks are predominantly produced in 
$\mathrm{W} \rightarrow \mathrm{W}$
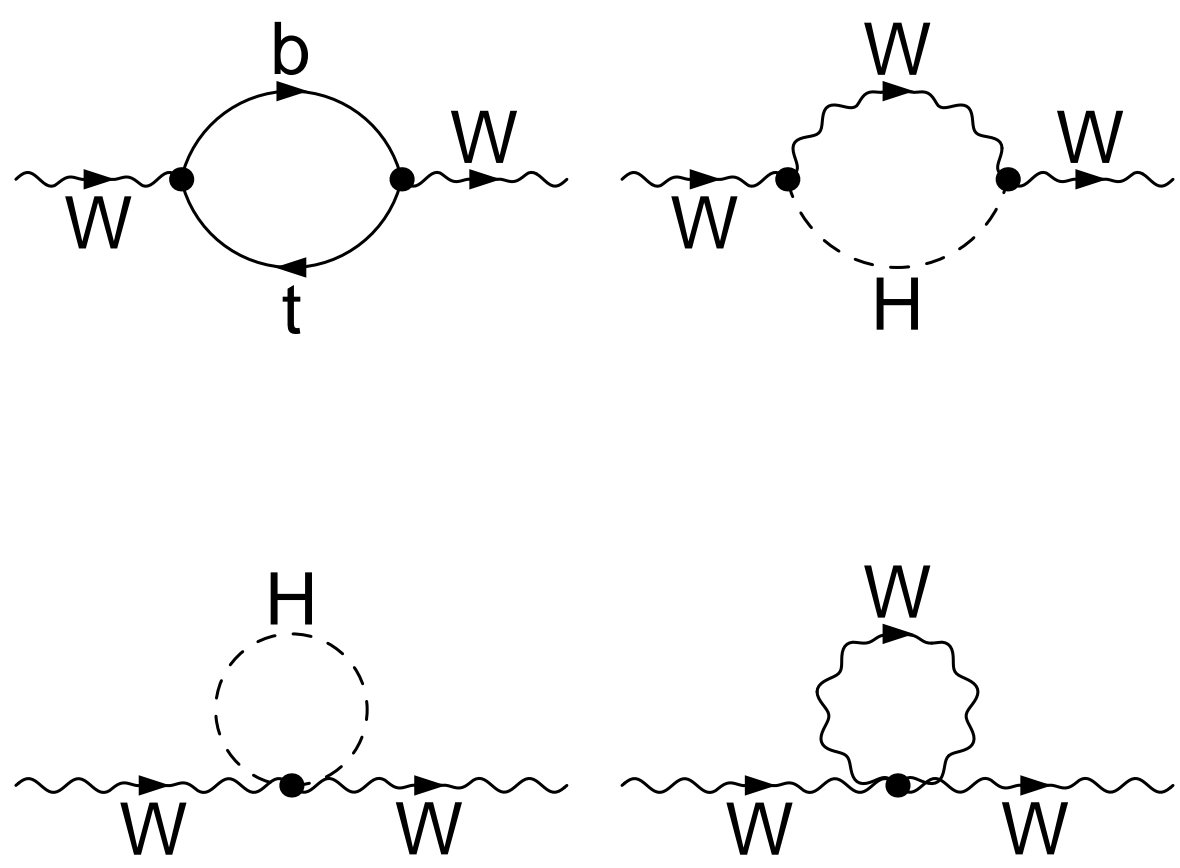

Figure 1.7: Electroweak radiative corrections to the $W$ boson propagator: these corrections are proportional to $m_{t}^{2}$ and $\ln m_{H}$. 


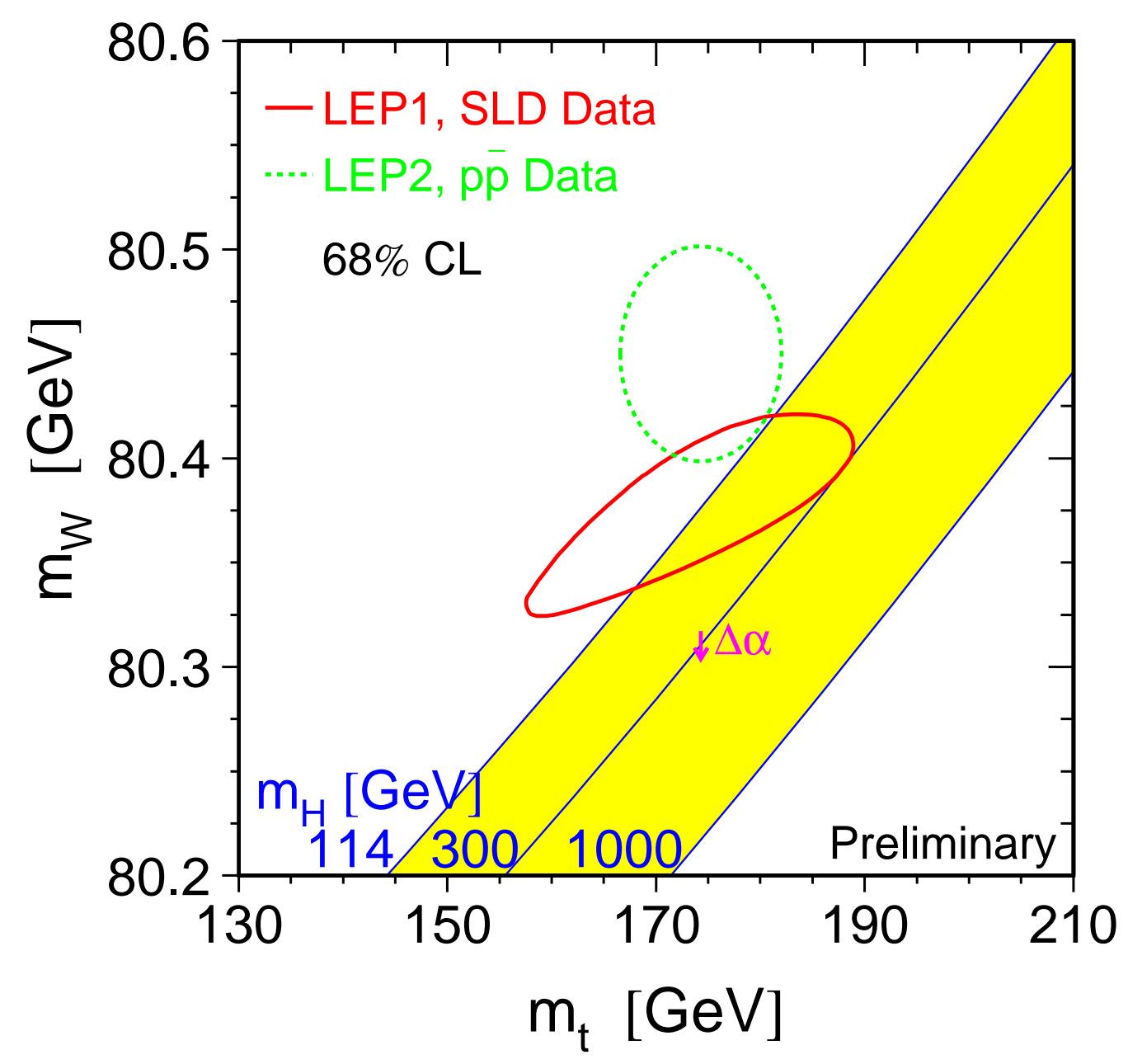

Figure 1.8: The comparison of the indirect measurements of $M_{W}$ and $M_{t}$ (LEP I+ SLD data) (solid contour) and the direct measurements ( $p \bar{p}$ colliders and LEP II data) (dashed contour). In both cases the 68\% CL contours are plotted. Also shown is the Standard Model relationship for the masses as a function of the Higgs mass. The arrow labeled $\Delta \alpha$ shows the variation of this relation if $\alpha\left(M_{Z}^{2}\right)$ is changed by one standard deviation. This variation gives an additional uncertainty to the Standard Model band shown in the figure [hep/ex-0312023]. 

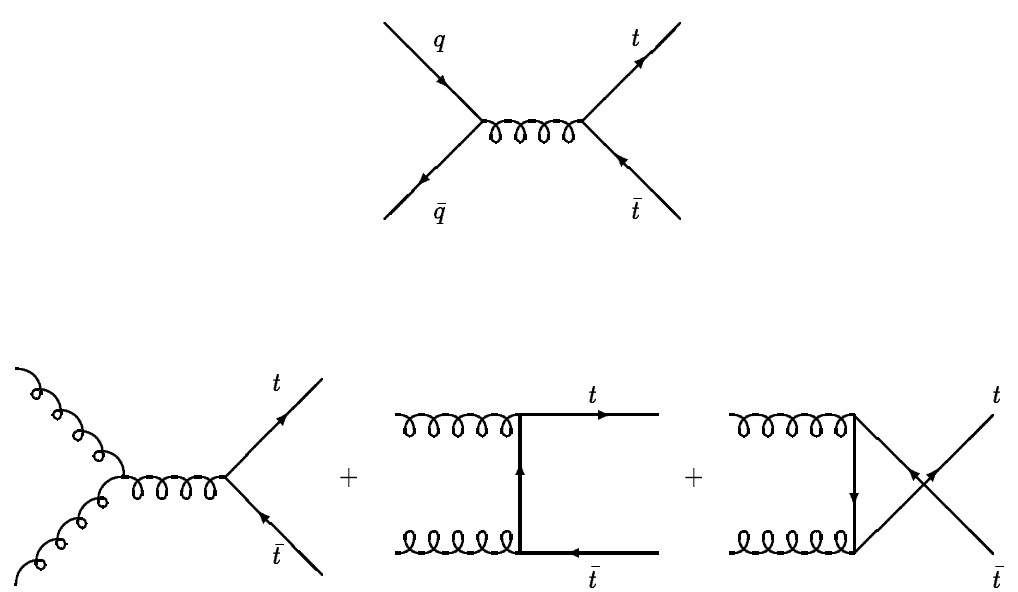

Figure 1.9: Feynman diagrams of pair production of top quarks at hadron colliders.

pairs. In $p \bar{p}$ collisions, strong interaction leads to the production of top and antitop $(t \bar{t})$ quarks. The electroweak production of top quark has not been observed yet experimentally. $t \bar{t}$ pairs are produced through two distinct processes - quark-antiquark annihilation $(q \bar{q} \rightarrow t \bar{t})$ and gluon-gluon fusion $(g g \rightarrow t \bar{t})$. The leading order (LO) Feynman diagrams are shown in Fig. 1.9. At Tevatron, $85 \%$ of the $t \bar{t}$ pairs are produced through quark annihilation while the contribution from gluon fusion is $15 \%$. The production cross section for $p \bar{p} \rightarrow t \bar{t}+\mathrm{X}$ can be calculated using perturbative QCD. The total tree level (Born Approximation) cross section at hadron colliders can be written as the convolution of the parton distribution functions (PDF's) for the in- 
coming hadrons (proton or antiproton) and the cross section for the partonic processes [25]:

$$
\sigma_{p \bar{p} \rightarrow t \bar{t}+X}=\sum_{a, b} \int d x_{a} F_{a}^{p}\left(x_{a}, \mu_{f}^{2}\right) \int d x_{b} F_{b}^{\bar{p}}\left(x_{b}, \mu_{f}^{2}\right) \hat{\sigma}_{a b}\left(\hat{s}, m_{t}, \alpha_{s}\left(\mu_{r}^{2}\right)\right)
$$

where $\mathrm{a}, \mathrm{b}$ are the possible combinations of the incoming gluon or quarkantiquark pairs, $F_{a}^{p}$ and $F_{b}^{\bar{p}}$ are the parton distribution function functions for the light partons $(\mathrm{u}, \mathrm{d}, \mathrm{c}, \mathrm{s}, \mathrm{b})$ which carry momentum fractions $x_{a}$ and $x_{b}$ of proton and antiproton respectively and are evaluated at the factorization scale, $\mu_{f}\left(\sim m_{t}\right)$. The parton level cross sections $\hat{\sigma}_{a b}$, for the process $a+b \rightarrow t \bar{t}$ $(q \bar{q} \rightarrow t \bar{t}$ or $g g \rightarrow t \bar{t})$ integrated over phase space, are functions of centreof-mass energy $\hat{s}$ at the parton level, top quark mass and the QCD strong coupling constant $\alpha_{s}$ at a renormalization scale $\mu_{r}$. Fig. 1.10 shows the parton distribution functions $(\mathrm{PDF})$ in proton for all the different species of partons. The parton distribution functions also depend on the relevant scale of the process, $\mu_{f}$, which for top quark production is of order $m_{t}$. For a typical value of $x$ at the Tevatron i.e., $x \approx 0.18$, the magnitude of PDF for the up quark is larger than that of the gluon, and the magnitude of PDF for the down quark is comparable to it. This explains why quark-antiquark annihilation dominates at the Tevatron. In contrast, for the typical value of 


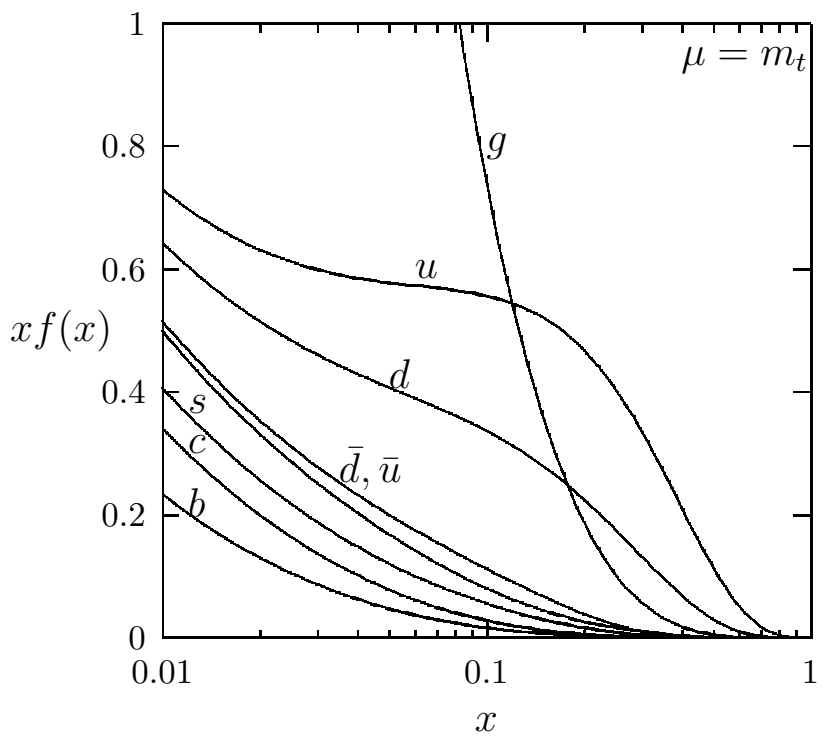

Figure 1.10: Parton distribution functions of a proton at the scale $\mu=m_{t}$, relevant for top-quark production. The probability of finding a given parton species with momentum fraction between $x\left(=x_{a} \approx x_{b}=x\right.$ where $\left.x \approx \frac{2 m_{t}}{\sqrt{\hat{s}}}\right)$ and $x+d x$ is $f(x) d x$. 


\begin{tabular}{c|c|c|c}
\hline & $\sigma_{\mathrm{NLO}}(\mathrm{pb})$ & $q \bar{q} \rightarrow t \bar{t}$ & $g g \rightarrow t \bar{t}$ \\
\hline Tevatron $(\sqrt{s}=1.8 \mathrm{TeV} p \bar{p})$ & $4.87 \pm 10 \%$ & $90 \%$ & $10 \%$ \\
Tevatron $(\sqrt{s}=2.0 \mathrm{TeV} p \bar{p})$ & $6.70 \pm 10 \%$ & $85 \%$ & $15 \%$ \\
LHC $(\sqrt{s}=14 \mathrm{TeV} p p)$ & $803 \pm 15 \%$ & $10 \%$ & $90 \%$ \\
\hline \hline
\end{tabular}

Table 1.2: Theoretical cross sections, at next-to-leading-order in QCD, for top-quark production via the strong interaction at the Tevatron and the LHC [26]. Also shown is the percentage of the total cross section from the quark-antiquark-annihilation and gluon-fusion subprocesses.

$x$ at the LHC, $x \approx 0.025$, gluon fusion dominates. Table 1.2 summarizes these results.

The matrix elements for the tree-level diagrams are:

$$
\begin{aligned}
\sigma(q \bar{q} \rightarrow t \bar{t})= & \frac{2}{9} \frac{4 \pi \alpha_{s}^{2}}{3 \hat{s}}\left(1+\frac{1}{2} \gamma\right)(1-\gamma)^{\frac{1}{2}} \\
\sigma(g g \rightarrow t \bar{t})= & \frac{\pi \alpha_{s}^{2}}{3 \hat{s}}\left[\left(1+\gamma+\frac{1}{16} \gamma^{2}\right) \ln \frac{1+(1-\gamma)^{1 / 2}}{1-(1-\gamma)^{1 / 2}}\right. \\
& \left.-\left(\frac{7}{4}+\frac{31}{16} \gamma\right)\left(1-\gamma^{2}\right)^{1 / 2}\right],
\end{aligned}
$$

where $\gamma=4 m_{t}^{2} / \hat{s}$ and $\hat{s}$ is the center-of-mass energy of incoming partons [27]. In perturbation theory, Next-to-leading order (NLO) contribution should be small compared to Leading order (LO). However, for $t \bar{t}$ production at Tevatron the NLO contributions are large because the processes involve soft gluon emission and it is necessary to include these contributions of higher order to have agreement with the experimental results. By construction, the results 
including all orders of perturbative QCD should be independent of the choice of scale $\left(\mu_{r}\right.$ and $\left.\mu_{f}\right)$, but are almost impossible to calculate. All practical calculations are performed to a finite order and the scale dependence is not realized for a finite order calculations. Varying the scale at a given order gives an idea of the residual uncertainty in calculation e.g., variation of $\mu_{r}\left(\mu_{f}\right)$ between $\frac{1}{2} m_{t}$ and $2 m_{t}$.

Higher order calculations e.g., $\mathrm{NLO}\left[\mathcal{O}\left(\alpha_{s}^{3}\right)\right]$, NNLO $\left[\mathcal{O}\left(\alpha_{s}^{4}\right)\right]$ etc., involve both real parton emission and virtual (loop diagrams) corrections. These corrections take the form of logarithms $\left[\ln ^{l}\left(x_{\mathrm{th}}\right) / x_{\mathrm{th}}\right]$ with $l \leq 2 n-1$ for the order $\alpha_{s}^{n}$ calculations, where $x_{\mathrm{th}}$ is a kinematic variable that measures the distance from threshold $\left(\frac{4 m_{t}^{2}}{\hat{s}}\right)$ and goes to zero at threshold. The logarithms raised to the power $l \equiv 2 n-1$ are the leading logarithms (LL), those with $l \equiv 2 n-2$ are next-to-leading logarithms (NLL), with $l \equiv 2 n-3$ are next-to-next-to-leading logarithms (NNLL) and so on. These logarithms can be summed to all orders of perturbation theory. The technique used for such summing is called the "resummation" technique [28].

The $t \bar{t}$ production cross section at Tevatron with $\sqrt{s}=1.96 \mathrm{TeV}$, has been estimated at NLO[29] and NNLO-NNNLL [30] using resummation techniques. In the NNLO-NNNLL calculation, the factorization/renormalization scale dependence of the cross section has been reduced. Uncertainty due to 


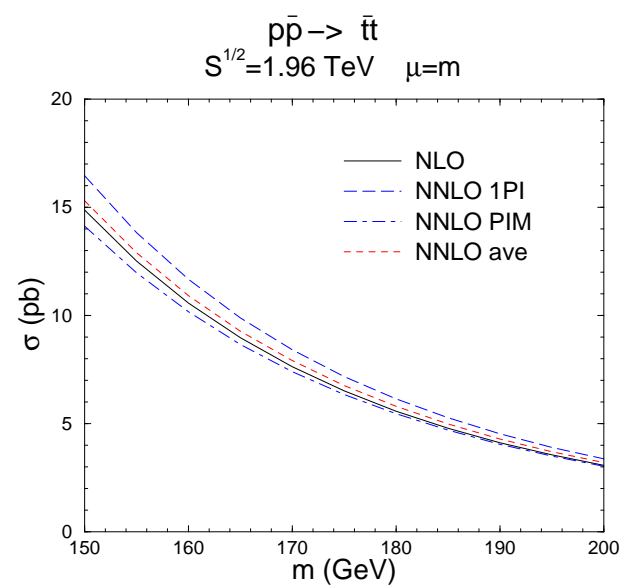

(a)

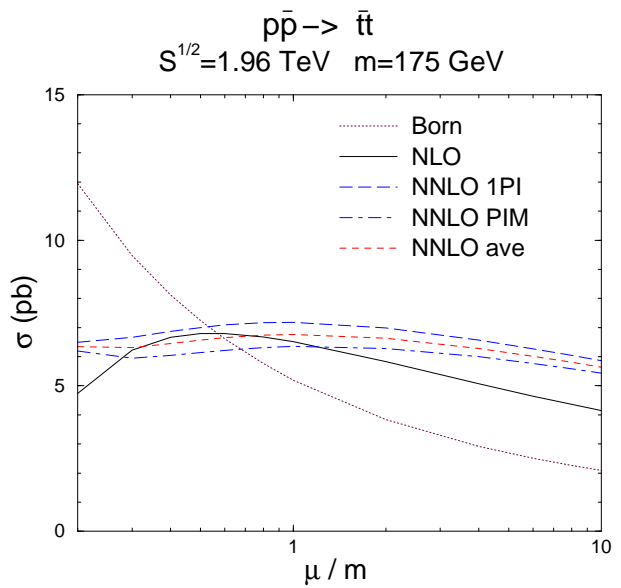

(b)

Figure 1.11: The $t \bar{t}$ production cross section (a) as a function of top quark mass at $\sqrt{s}=1.96 \mathrm{TeV}$, evaluated at a scale $\mu=m_{t}$, (b) as a function of $\mu / m_{t}$, for $m_{t}=175 \mathrm{GeV} / \mathrm{c}^{2}$. The difference in the choice of kinematics viz., PIM, 1PI is also shown (see Ref. [30]).

the choice of scale is almost negligible while the choice of kinematics (see Fig. 1.11) imposes some uncertainty near threshold. The latest theoretical status of $t \bar{t}$ cross section is shown in Fig. 1.11 (a, b), as functions of top quark mass and variation in scale, respectively.

\subsubsection{Decay of Top Quark}

A particle's lifetime is the inverse of its decay width, $\tau=\frac{\hbar}{\Gamma}$. The partial decay width of the top quark $\left(t \rightarrow b W^{+}\right)$, can be calculated by considering the LO Feynman diagram in Fig. 1.12. Neglecting the non-zero mass of $b$ 


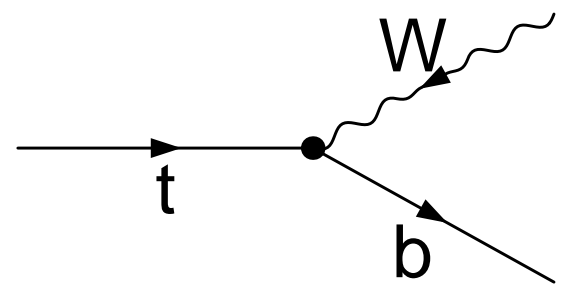

Figure 1.12: Feynman diagram for $t \rightarrow b W^{+}$from which the decay width of the top quark $\left(\Gamma_{\mathrm{t}}\right)$ can be calculated to leading order.

quark and the width of $W^{+}$boson, the LO $t \rightarrow b W^{+}$partial width is given by

$$
\Gamma\left(t \rightarrow b W^{+}\right)=\frac{G_{F}}{8 \pi \sqrt{2}} m_{t}^{3}\left|V_{t b}\right|^{2}\left(1-3 \frac{M_{W}^{4}}{m_{t}^{4}}+2 \frac{M_{W}^{6}}{m_{t}^{6}}\right)=1.56 \mathrm{GeV} .
$$

The NLO result is $1.42 \mathrm{GeV}$ [31]. This leads to an extremely short lifetime, about $4 \times 10^{-25} \mathrm{~s}$ in the SM, which is $\mathcal{O}(10)$ times shorter than the characteristic hadronization time of QCD, $\tau_{\text {had }} \approx 28 \times 10^{-25} \mathrm{~s}$. As a result, the decay of top quark offers an unique opportunity to study the bare quark, free from the long-range effects of QCD, such as confinement. The $W^{+}$boson is a real one because $m_{t}>M_{W^{+}}+M_{b}$. This is a weak flavour changing charge current $($ FCCC $)$ decay with a branching fraction $(\mathcal{B})>99.8 \%$. Other FCCC decays, such as $t \rightarrow W^{+} s$ and $t \rightarrow W^{+} d$ are also allowed, but they are suppressed by factors of $\mathcal{O}\left(10^{-3}\right)\left[\mathrm{t} \rightarrow \mathrm{W}^{+} \mathrm{s}\right]$ and $\mathcal{O}\left(10^{-4}\right)\left[\mathrm{t} \rightarrow \mathrm{W}^{+} \mathrm{d}\right]$ through the mixing 
elements of CKM matrix [32]. FCCC "radiative" decay, viz., $t \rightarrow W^{+} b Z^{0}$ is also possible with a branching fraction of $\mathcal{O}\left(10^{-7}-10^{-6}\right)$. The decays of top quark via flavour changing neutral current (FCNC), are also allowed in SM with the predictions for $\mathcal{B}(t \rightarrow c g) \sim 4 \times 10^{-13}, \mathcal{B}(t \rightarrow c \gamma) \sim 5 \times 10^{-13}$, and $\mathcal{B}\left(t \rightarrow c Z^{0}\right) \sim 1 \times 10^{-13} . \mathcal{B}\left(t \rightarrow c H^{0}\right)$ depends on the Higgs mass $\left(M_{H^{0}}\right)$, but it cannot exceed $\sim 10^{-13}$. A comprehensive review regarding the latest status of top quark can be found in Ref. [32, 33].

\subsubsection{Experimental Signature}

This dissertation considers only the exclusive SM decay of top quark, $t \rightarrow$ $W^{+} b$. The $W$ boson has a lifetime $\sim 3 \times 10^{-25}$ s and almost instantaneously decays either leptonically into a lepton-neutrino pair, $W \rightarrow l \overline{\nu_{l}}$ with $l=$ $e, \mu, \tau$ or hadronically into a quark-antiquark pair, $W \rightarrow q_{1} \overline{q_{2}}$, with $q_{1}\left(q_{2}\right)=$ $u(d), c(s)$ Tab. 1.3 shows the corresponding branching fractions. Hadronic final states result in showers of particles, called jets. The leptonic decays of $W$ with the exception of $\tau$-lepton ${ }^{\S}$, can be identified through the detection of a charged lepton, while the associated neutrino escapes direct detection. Fragmentation and hadronization of the $b$ quark on the other hand, produces

\footnotetext{
$\S$ It is very tough to detect the $\tau$-lepton experimentally, as it decays immediately into hadronic or leptonic final states. One needs sophisticated identification methodology to detect the $\tau$ 's depending on the characteristics of the detector.
} 


\begin{tabular}{|c|c|}
\hline Final State & Branching Fraction \\
\hline$e \nu_{e}$ & $10.72 \%$ \\
$\mu \nu_{\mu}$ & $10.57 \%$ \\
$\tau \nu_{\tau}$ & $10.74 \%$ \\
$u d$ & $33.98 \%$ \\
$c s$ & $33.98 \%$ \\
\hline
\end{tabular}

Table 1.3: Decay modes of $W^{ \pm}$and its branching fractions [32].

the signature of a jet.

Since there are $2 W^{\prime}$ 's in a $t \bar{t}$ event, the event topologies are named according to how the two $W^{\prime}$ 's decay. When both $W$ 's decay into jets (all jets channel), branching fraction $(46 \%)$ is the highest, but this channel suffers from the enormous QCD multi-jet background. When one $W$ boson decays leptonically and the other hadronically $(e+$ jets and $\mu+$ jets $)$ the branching fractions $(17 \%)$ are reasonable and the background contribution is smaller than the all jets channel. Statistically significant measurements of both, mass of top quark and its production cross section, have been performed using these two channels, during Run I of Tevatron. The dilepton channels (total BR of $6.4 \%$ ), where both $W$ 's decay leptonically, are the cleanest channels but they suffer because of low branching fractions. A graphical representation of various SM branching fractions for the decays of top-antitop pairs is shown in Fig. 1.13. 


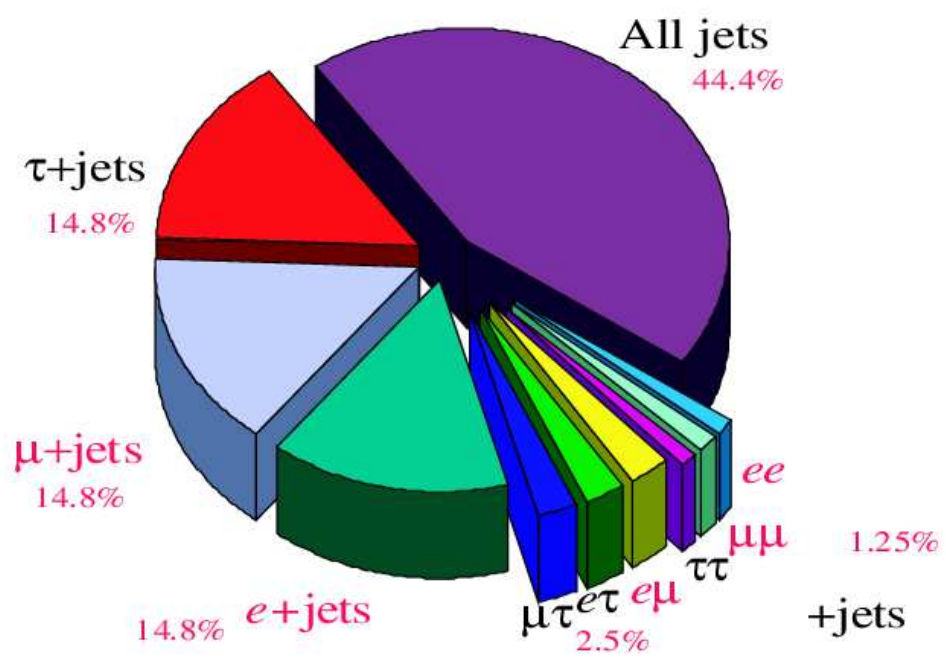

Figure 1.13: Breakdown of $t \bar{t}$ events into various final states and their branching fractions.

\subsection{The $e \mu$ channel}

Among the dileptonic decay modes of $t \bar{t}$, the $e \mu$ decay mode has the largest branching ratio. Moreover, this channel is the least contaminated by $Z^{0} / \gamma^{*}$ production, which is the dominating backgrounds for other dilepton channels ${ }^{\ddagger}$ viz., ee and $\mu \mu$. This dissertation presents a measurement of $t \bar{t}$ production cross section using the decay channel $t \bar{t} \rightarrow e \mu$.

\footnotetext{
$\ddagger$ At the current centre of mass energy available at Tevatron, the production cross section for the $Z^{0} / \gamma^{*} \rightarrow l^{+} l^{-}$(with $\left.l=e, \mu, \tau\right)$ is $\approx 100$ times higher than that for $t \bar{t} \rightarrow e^{+} e^{-} / \mu^{-} \mu^{+}$process. Since electrons/muons coming from the decay of massive $Z^{0}$ boson are highly energetic, they appear almost identical to the ones coming from $t \bar{t}$ decays.
} 


\subsubsection{Event Signature}

Characteristics of the $e \mu$ decay channel are

- One electron with large transverse energy $\left(p_{T}^{e}\right)$ from W-decay

- One muon with large transverse momentum $\left(p_{T}^{\mu}\right)$ from $\mathrm{W}$-decay

- Missing transverse momentum $\left(E_{T}\right)$ carried away by the neutrinos from the $W^{ \pm}$-decays

- $\geq 2$ jets, from the fragmentation of two b-quarks and from initial state and final state radiations

Apart from the direct decays $t \bar{t} \rightarrow W^{+} b W^{-} \bar{b} \rightarrow e \nu_{e} \mu \nu_{\mu} b \bar{b}, t \bar{t}$ can also reproduce the $e \mu$ signature through the subsequent decay(s) of $\tau$ lepton(s) i.e.,

- $t \bar{t} \rightarrow W^{+} b W^{-} \bar{b} \rightarrow e \nu_{e} \tau \nu_{\tau} b \bar{b} \rightarrow e \nu_{e} \mu \nu_{\mu} \nu_{\tau} b \bar{b}$

- $t \bar{t} \rightarrow W^{+} b W^{-} \bar{b} \rightarrow \mu \nu_{\mu} \tau \nu_{\tau} b \bar{b} \rightarrow \mu \nu_{\mu} e \nu_{e} \nu_{\tau} b \bar{b}$

- $t \bar{t} \rightarrow W^{+} b W^{-} \bar{b} \rightarrow \tau \nu_{\tau} \tau \nu_{\tau} b \bar{b} \rightarrow \mu \nu_{\mu} \nu_{\tau} e \nu_{e} \nu_{\tau} b \bar{b}$

Diagrams for all such decays are shown in Fig. 1.14 having a total branching fraction of $3.16 \%[32]$ for $t \bar{t} \rightarrow e \mu$ signal. 


\subsubsection{Background}

In high energy physics experiment, one tries to identify events, arising from a process specified by the final state patricles e.g., $t \bar{t} \rightarrow e \mu$. Along with the signal events, the experimental data contain events from many other processes which can reproduce the same signature. All such processes which can potentially contaminate the signal events are called the backgrounds, background decays or background channels. The background processes for $t \bar{t} \rightarrow e \mu$ signal can be categorised into two major classes - Physics and Instrumental.

\section{Physics}

The primary sources of background having $e \mu$ final state, are contributed by $Z^{0} / \gamma^{*} \rightarrow \tau^{+} \tau^{-} \rightarrow e \mu$ and $W^{+} W^{-} \rightarrow e \mu$ decays. These background processes consist of a real electron, a real muon and neutrinos along with the presence of jets.

1. $\mathbf{Z}^{\mathbf{0}} / \gamma^{*}$ can decay into a $\tau^{+} \tau^{-}$pair which subsequently decay into an electron amd a muon. The leading order Feynman diagram for this decay is shown in Fig. 1.15. This is the largest background process at the production level, having a cross section $\sim 100$ times higher than 
that of $t \bar{t}$ production. But both the leptons (and the neutrinos) in this case, have lower transverse momenta compared to those coming from the $t \bar{t}$ decay. The jets in these events are typically produced through radiative processes and also have smaller transverse energy $\left(\mathrm{E}_{\mathrm{T}}\right)$.

2. $\mathbf{W}^{+} \mathbf{W}^{-}$pairs are produced at the Tevatron through Drell-Yan and tchannel processes (see Fig. 1.16). The subsequent leptonic decays of W bosons can contaminate the $t \bar{t} \rightarrow e \mu$ signal. The inclusive production cross section for $W^{+} W^{-}$at $\sqrt{s}=1.96 \mathrm{TeV}$ is of the same order as that of $t \bar{t}$ cross section. Since the leptons as well as neutrinos come from W-decays, their transverse momentum spectra are very similar to those of $t \bar{t}$ events. Similar to the $Z^{0} / \gamma^{*} \rightarrow \tau \tau$ decays, the jets in these events are produced through radiative processes and show softer transverse energy spectra, as compared to the jets coming from $t \bar{t}$ decays.

3. $\mathbf{W}^{ \pm} \mathbf{Z}^{\mathbf{0}}$ production can also contaminate the $t \bar{t} \rightarrow e \mu$ signal when both bosons decay as, $W^{ \pm} Z^{0} \rightarrow \mu^{ \pm} \nu_{\mu} e^{+} e^{-}$or $W^{ \pm} Z^{0} \rightarrow e^{ \pm} \nu_{e} \mu^{+} \mu^{-}$. Because of very low branching ratio for these two decays, the contamination from this background is almost negligible. 


\section{Instrumental}

Instrumental backgrounds are the ones which are produced due to detector effects and lepton misidentification. There are two types of such backgrounds as mentioned below.

- Photon backgrounds can contaminate the $e \mu$ signal when a photon (either a real one produced directly in $p \bar{p}$ collisions or a bremsstrahlung emitted by an energetic muon during its passage through the detector) gets misidentified as an electron candidate. Associated photon production of weak vector bosons i.e., $W^{ \pm} \gamma$ or $Z^{0} \gamma$ production with $W^{ \pm} / Z^{0}$ decaying into muons and $Z^{0} / \gamma^{*} \rightarrow \mu^{+} \mu^{-}$, where one of the muons emits a bremsstrahlung photon in the detector, can mimic the $t \bar{t} \rightarrow e \mu$ event topology.

- Misidentification of leptons can occur for processes like QCD (producing multiple jets $), W^{ \pm}\left(\rightarrow \mu \nu_{\mu}\right)+$ jets, $W^{ \pm}\left(\rightarrow e \nu_{e}\right)+\mathrm{b} \overline{\mathrm{b}}$ events where an object is identified as an isolated lepton (could be an electron or a muon) by mistake. In the case of $\mathrm{QCD}$ and $W^{ \pm}\left(\rightarrow \mu \nu_{\mu}\right)+$ jets events, this can occur when a jet is mis-reconstructed as an electron candidate. On the other hand, in $W^{ \pm}\left(\rightarrow e \nu_{e}\right)+\mathrm{b} \bar{b}$ events, a muon (from the semi-leptonic decay of a b quark) can appear to be isolated from jets, 
if the jet surrounding the muon is not reconstructed. Since the transverse momenta of these events are generally mismeasured, these events can result in large missing transverse energy and can contaminate the $t \bar{t} \rightarrow e \mu$ signal. This type of background processes is also called fake background in the later chapters of this dissertation. 


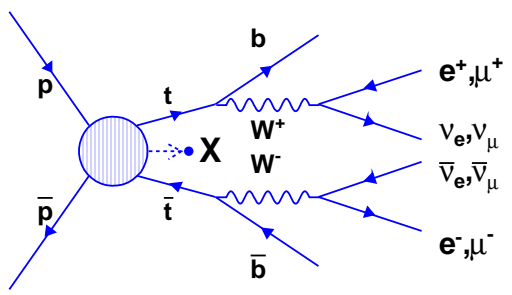

(a)

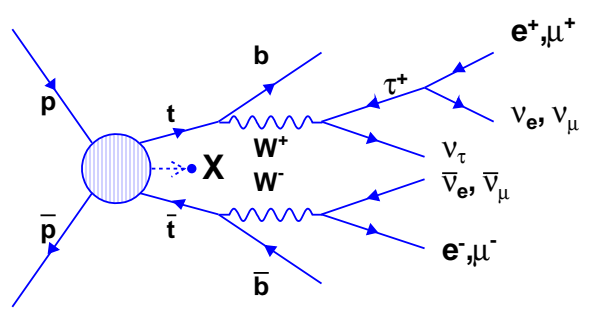

(b)

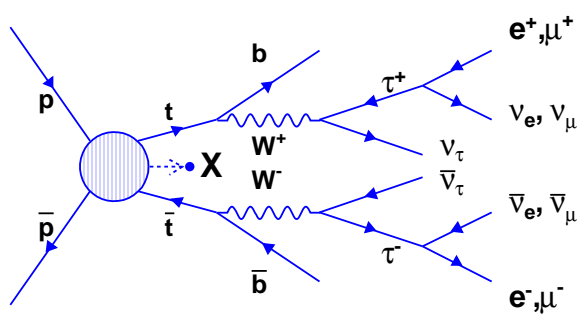

(c)

Figure 1.14: The schematics of various $t \bar{t} \rightarrow e \mu$ decays: (a) $t \bar{t} \rightarrow W^{+} b W^{-} \bar{b}$ $\rightarrow e \nu_{e} \mu \nu_{\mu} b \bar{b},(\mathrm{~b}) t \bar{t} \rightarrow W^{+} b W^{-\bar{b}} \rightarrow e \nu_{e} \tau \nu_{\tau} b \bar{b}\left(\mu \nu_{\mu} \tau \nu_{\tau} b \bar{b}\right) \rightarrow e \nu_{e} \mu \nu_{\mu} \nu_{\tau} b \bar{b}$ $\left(\mu \nu_{\mu} e \nu_{e} \nu_{\tau} b \bar{b}\right),(\mathrm{c}) t \bar{t} \rightarrow W^{+} b W^{-} \bar{b} \rightarrow \tau \nu_{\tau} \tau \nu_{\tau} b \bar{b} \rightarrow \mu \nu_{\mu} \nu_{\tau} e \nu_{e} \nu_{\tau} b \bar{b}$. 


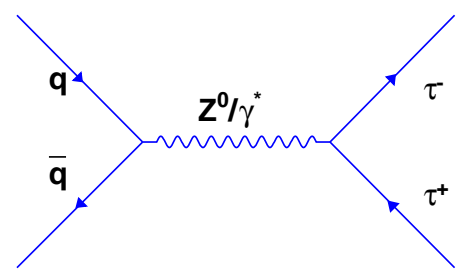

Figure 1.15: Leading order diagram for $Z^{0} / \gamma^{*} \rightarrow \tau^{+} \tau^{-}$at Tevatron.

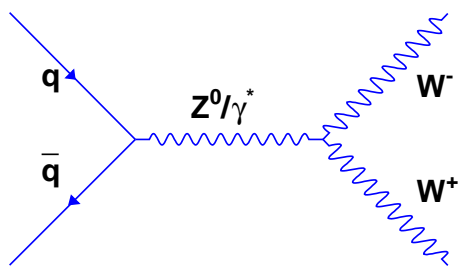

(a)

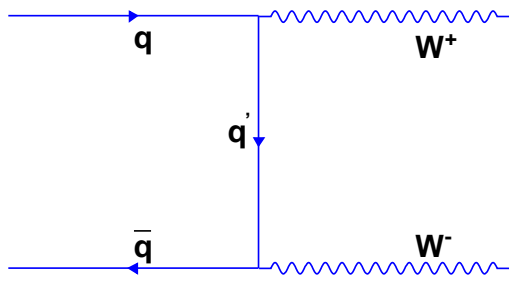

(b)

Figure 1.16: Leading order diagram for $W^{+} W^{-}$production at Tevatron: (a) s-channel and (b) t-channel. 


\section{Chapter 2}

\section{Experimental Apparatus}

The data presented in this dissertation, have been produced using the Tevatron proton-antiproton collider at Fermilab (USA) and are recorded by the DØ detector. At present, Tevatron is the world's highest energy collider where beams of protons and antiprotons collide at a centre-of-mass energy $(\sqrt{s})$ of $1.96 \mathrm{TeV}$. The protons $(p)$ and antiprotons $(\bar{p})$ are accelerated in a series of accelerators and are injected into the Tevatron ring where each of them is accelerated to an energy of $980 \mathrm{GeV}$. Inside the Tevatron ring, protons and antiprotons rotate in opposite directions and collisions take place at two points around the circumference of the ring. $\mathrm{D} \varnothing$ detector is situated at one of these collision points. At the other collision point, another experiment called CDF (Collider Detector at Fermilab), is located. The following sec- 
tions of this chapter describe the main components of Tevatron accelerator and $\mathrm{D} \varnothing$ detector.

\subsection{Tevatron Accelerator System}

Tevatron, a $p \bar{p}$ collider was built in 1985 to provide collisions at $\sqrt{s}=1.8 \mathrm{TeV}$, only to the CDF collider experiment, while DØ experiment started in 1992. After the glorious Run I period of 1993-1996, Tevatron accelerator system has been upgraded to increase the beam energy and luminosity ${ }^{\ddagger}$. Upgraded Tevatron accelerator system consists of: (1) Cockroft-Walton Pre-accelerator, (2) Linear Accelerator (LINAC), (3) Booster Synchrotron, (4) Main Injector, (5) Antiproton source, (6) Antiproton recycler, and (7) Tevatron ring. A schematic diagram of the Tevatron complex is shown in Fig. 2.1. The procedure for production and acceleration of protons and antiprotons is described below.

\subsubsection{Proton Production}

Production of proton beam starts with the injection of hydrogen gas into an "ion source" (see Fig. 2.2), where $\mathrm{H}^{-}$ions are created in the presence of

\footnotetext{
$\ddagger$ The beam luminosity or instantaneous luminosity is the rate of $p \bar{p}$ collisions per unit area, usually expressed in $\mathrm{cm}^{-2} \mathrm{sec}^{-1}$.
} 


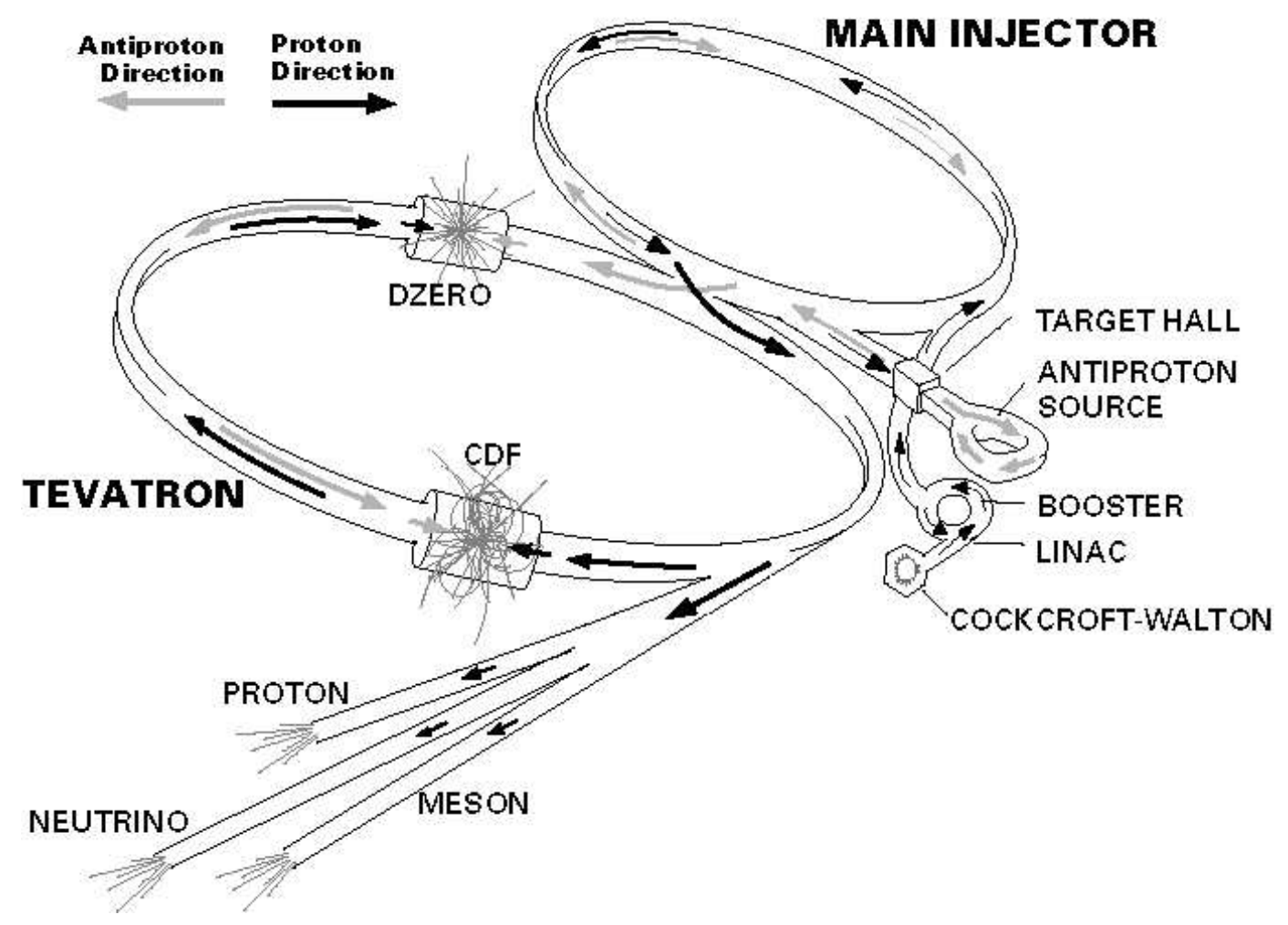

Figure 2.1: Tevatron accelerator complex at Fermilab with two collider detectors viz., CDF and DØ. 
a catalytic surface, made of Cesium ${ }^{\S}$. These $\mathrm{H}^{-}$ions are released into the Cockroft-Walton pre-accelerator and are accelerated electro-statically [34]. The high voltage is achieved by applying low voltage (75 kilovolt) to a configuration of capacitors, charging them in parallel and then discharging them in series. The $\mathrm{H}^{-}$ions are accelerated by the Cockroft-Walton machine to a kinetic energy of $750 \mathrm{KeV}$. The pre-accelerator produces a continuous supply of $\mathrm{H}^{-}$ions. The ribbon of ions passes through a device, called an Electrostatic Chopper that "chops" out a portion (a pulse) of the beam. The chopper acts like a scissor that clips a 42-microsecond length of ribbon off (the pulse) and continues to do this chopping at the frequency the LINAC uses. Each pulse goes through a Buncher, which forces the pulse into a bunch and then transfers each bunch, to the LINAC.

LINAC, a linear accelerator, is composed of two different types of accelerating components: drift tubes and side-coupled cavities. There are five drift tube cavities where an alternating electric field, with a radio frequency (RF) of $201.24 \mathrm{MHz}$, is applied and the drift-tubes accelerate the beam from $750 \mathrm{KeV}$ to $116 \mathrm{MeV}$. The Side-Coupled LINAC (SCL) is the second stage with seven cavities operating at RF of $805 \mathrm{MHz}$. SCL accelerates the $\mathrm{H}^{-}$ion

\footnotetext{
${ }^{\S}$ Cesium has low work function; thus free electrons get attached to the $\mathrm{H}^{+}$ion very easily.
} 


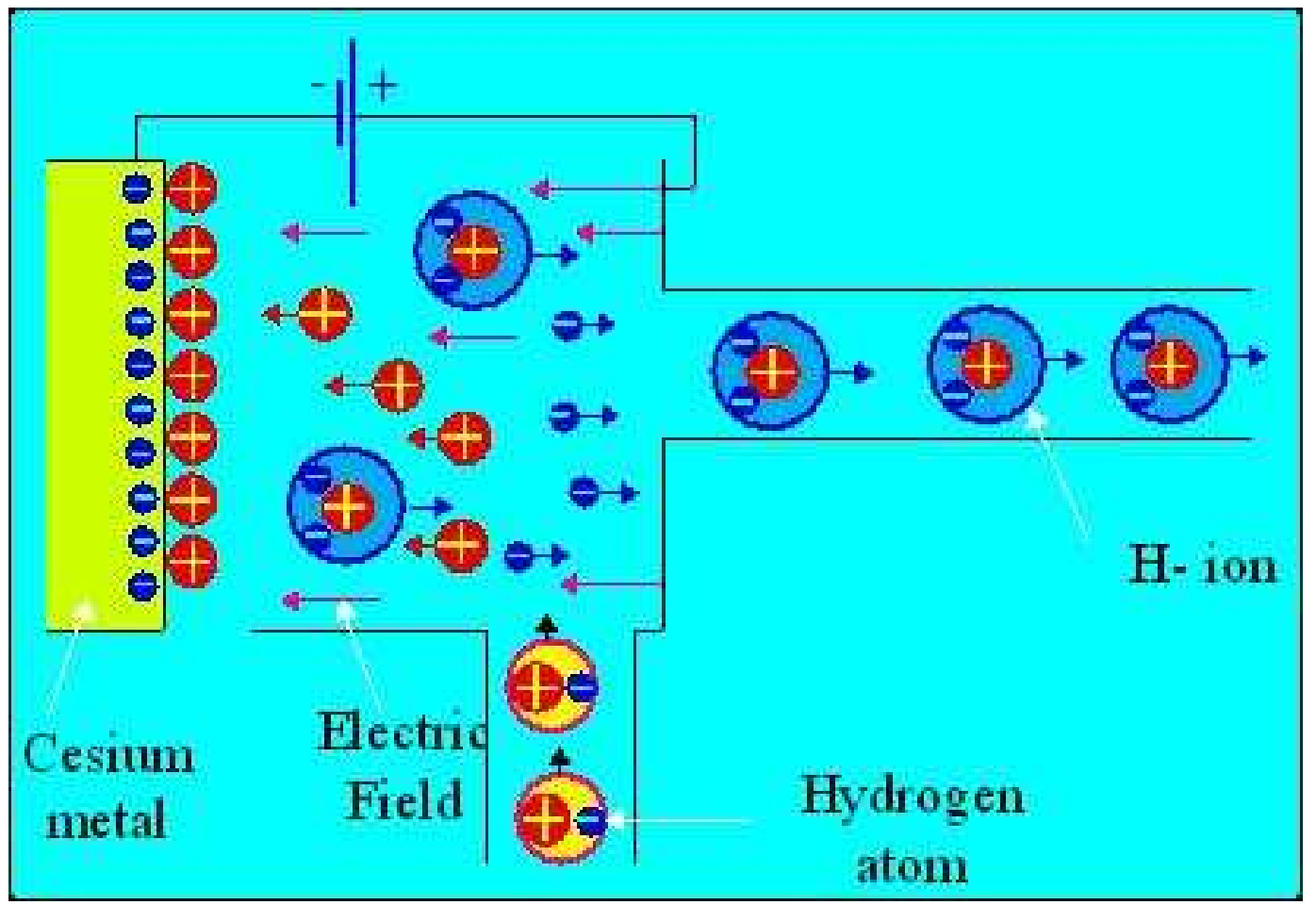

Figure 2.2: $\mathrm{H}^{-}$source: atoms of gaseous hydrogen gets ionised into a proton and an electron under the electric field and the proton congregates on metal surface. Sometimes a proton (drifting towards Cesium surface) smacks a proton with two electrons off the Cesium surface. 
beam to an energy of $400 \mathrm{MeV}$.

At this stage, the $\mathrm{H}^{-}$ions are sent through a carbon foil which strips off the electrons. The protons are then steered into the Booster synchrotron ring (approximately 500 metre in circumference). A synchrotron is a cyclic machine in which particles are confined to a closed orbit by a series of bending magnets. The particle's energy is increased by accelerating it in a synchronized RF cavity. As the momentum increases, the magnetic field in the bending magnet must be increased in order to keep the the particles inside the ring. Thus for a given ring, the maximum particle energy is limited by the maximum strength of the magnets. The Booster accelerates the protons to an energy of $8 \mathrm{GeV}$. The protons are then injected to the Main Injector.

Fermilab's Main Injector is a newly built synchrotron accelerator with a circumference of $\sim 3.3$ kilometres. Main Injector contains numerous conventional copper-coiled dipole (to bend the beam) and quadrupole (to focus the beam) magnets. There are $18 \mathrm{RF}$ cavities in the Main Injector which accelerate protons and antiprotons to $150 \mathrm{GeV}$, before injecting them into the Tevatron. The coalescing of proton or antiproton bunches into shorter bunches before injecting into the Tevatron ring, is also done in Main Injector. The Main Injector is also used to accelerate protons for the production of antiprotons. For antiproton production, the protons are accelerated to 
$120 \mathrm{GeV}$ in the Main Injector and the beam is taken to the Target Hall, as described in the next section.

\subsubsection{Antiproton Production}

Fig. 2.3 shows the general layout of the antiproton source at Fermilab. A $1.5 \mu \mathrm{sec}$ long pulse train of 82 bunches of $120 \mathrm{GeV}$ protons is focused on a nickel target. For every $10^{6}$ protons which strike the target, only about 20 antiprotons are created. These antiprotons have a large spread in energy. In order to focus the antiprotons, a cylinder of liquid lithium that transforms a current pulse of 120,000 amperes into a focusing magnetic field, is used. After passing through the lithium lens, the antiprotons are deflected by a pulsed dipole magnet which selects $8 \mathrm{GeV}$ antiprotons, for injection into the first one of the two antiproton storage rings, called the Debuncher. This is schematically shown in Fig. 2.3.

The antiprotons entering into the Debuncher, have a large spread in momentum and many are oscillating transverse to the beam direction (large emittance). The process whereby the momentum spread and emittance are reduced is known as "cooling". The Debuncher uses two cooling processes. The first method, called debunching, was invented at Fermilab. As a bunch of 


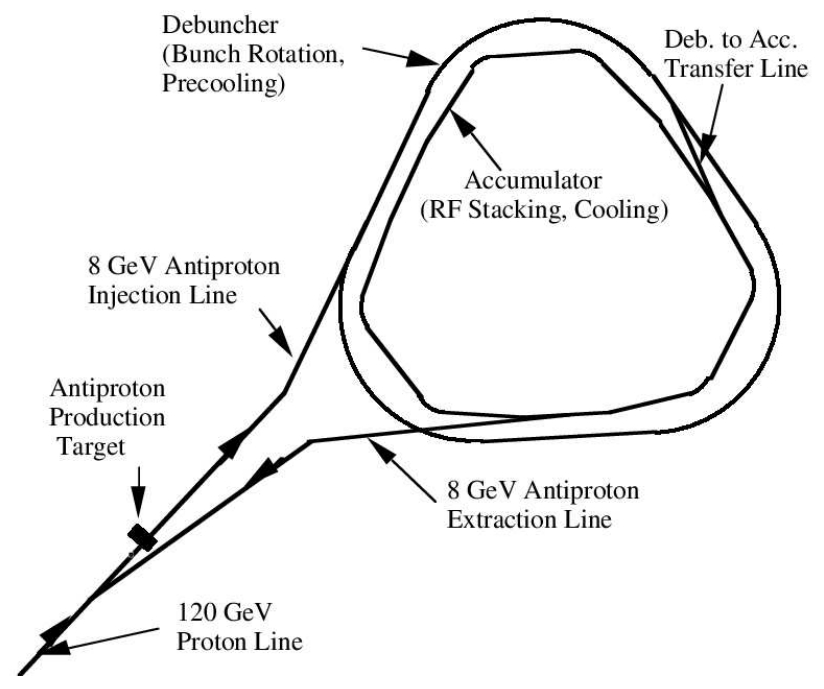

Figure 2.3: A layout of Antiproton source.

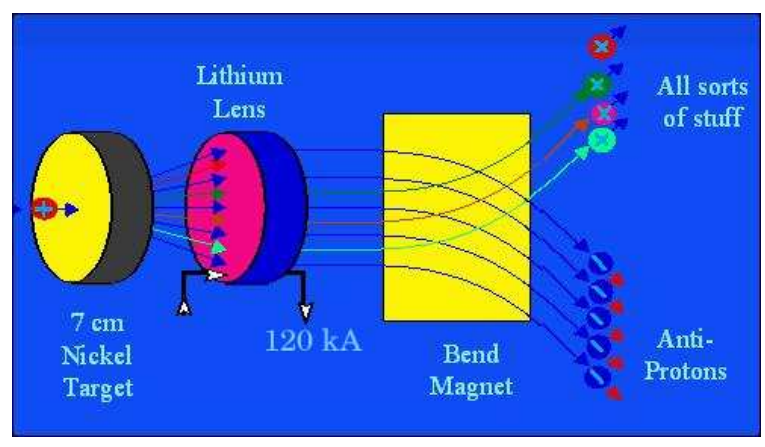

Figure 2.4: A diagram showing the components in the Antiproton target station. 


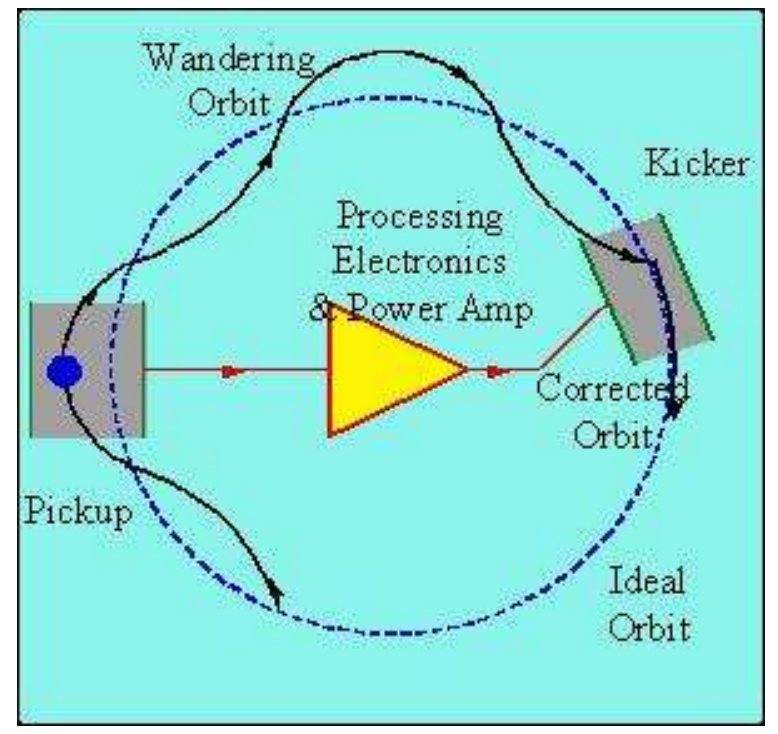

Figure 2.5: Stochastic Cooling of antiprotons, in Debuncher and Accumulator.

antiprotons circulates around the ring, computer controlled radio-frequency techniques act to smooth the antiprotons into a uniform continuous ring, where all the particles have approximately the same momenta. The second process, which restricts the transverse oscillations of the antiprotons, is known as "stochastic" cooling (see Fig. 2.5). Particles whose orbits are not ideal are identified by sensors which send correction signals in advance to kicker electrodes that on the average adjust the path of the wayward particle. When the process is completed, the antiprotons are in a single continuous ring at about the same momentum and with very little transverse spread. 
The above process runs continuously and about $\approx 10^{7}$ antiprotons are transferred at a time, into the second antiproton storage ring, called Accumulator. The Debuncher and Accumulator reside in the same tunnel of 520 m circumference as depicted in Fig. 2.3. The Accumulator provides further cooling to the antiprotons. After four to six hours, when the population of antiprotons in the Accumulator reaches about $80-200 \times 10^{10}$, the antiprotons are fed into the Main Injector and are accelerated to $150 \mathrm{GeV}$ before injection into the Tevatron ring.

The Antiproton Recycler is a fixed-energy storage ring placed in the Main Injector tunnel directly above the Main Injector beam-line. In order to achieve the luminosity goals of Run II, it is built to recycle the antiprotons from the Tevatron ring and to store the antiprotons from Accumulator. But the recycler is not yet in operation.

\subsubsection{The Tevatron Ring}

The Tevatron is the final synchrotron accelerator, having a radius of nearly 1 kilometre. It consists of superconducting bending magnets that are able to produce very high magnetic fields (operating at a temperature of $4.3^{0} \mathrm{~K}$, they produced a field of $\sim 4.2$ Tesla). The current flowing through a magnet 
in Tevatron to sustain proton or antiproton beams at $980 \mathrm{GeV}$, is more than 4000 Amperes. First, 36 bunches of antiprotons (typically $3.6 \times 10^{10}$ antiprotons/bunch) from the Main Injector, are loaded into the Tevatron ring and are kept at the Main Injector energy i.e., $150 \mathrm{GeV}$. After successful loading of antiprotons, 36 bunches of protons (typically $2.4 \times 10^{11}$ protons/bunch) are transferred to the Tevatron ring. During the process of loading protons or antiprotons, 36 bunches of them are placed in three symmetrically spaced super-bunches, each consisting of 12 bunches. Bunches within a super-bunch are separated by $396 \mathrm{~ns}$ while the super-bunches are separated by $\approx 2.64 \mu \mathrm{s}$ from each other. Once the $150 \mathrm{GeV}$ protons and antiprotons are loaded, both beams are accelerated to an energy of $980 \mathrm{GeV}$. After attaining full energy, transverse areas of the beams are squeezed at two beam crossing points B $\varnothing$ $(\mathrm{CDF})$ and $\mathrm{D} \varnothing$. The beam radius at these points is about $50 \mu \mathrm{m}$. The longitudinal beam size is quite large, resulting in the distribution of collisions along the beam direction to be roughly Gaussian with $\sigma \approx 25 \mathrm{~cm}$. Several particles travel with the beam usually at a slightly larger radius and thus create a halo around the beam. Before recording data from $p \bar{p}$ collisions, the beam halos are removed by a process known as "scraping", where the beams are collimated by metal plates. Typical beam lifetime is about 7 to 30 hours, depending on the luminosity requirements of both experiments, after which 
the beams are terminated by directing them into concrete blocks. In Run II, the typical instantaneous luminosity is around $0.86 \times 10^{32} \mathrm{~cm}^{-2} \mathrm{~s}^{-1}$. Detailed description of different components of Tevatron Run II collider can be found in Ref. [35].

\section{$2.2 \mathrm{D} \emptyset$ Detector}

$\mathrm{D} \varnothing$ is a multipurpose detector constructed to study the phenomena arising from $p \bar{p}$ collisions in Tevatron collider and has been operating since 1992. The prime focus of $\mathrm{D} \varnothing$ experiment is to study the high mass states and high transverse momentum phenomena arising in $p \bar{p}$ collisions. This includes study of top quark, search for Higgs boson, critical tests of the standard electroweak theory through precision studies of $\mathrm{W}^{ \pm} / \mathrm{Z}^{0}$ boson production and decay, various studies of perturbative QCD (pQCD), studies involving b-quarks as well as searches for new phenomena beyond the Standard Model. In order to accomplish these requirements, the detector has been optimized in terms of excellent identification of leptons (including neutrinos) and hadrons with great precision in their energy and momentum measurement.

After a satisfactory performance during Tevatron Run I (1992-96), various components of $\mathrm{D} \varnothing$ detector have been upgraded [36]. The gaseous track- 
ing chambers in the inner part of the detector, have been replaced by a new tracking system containing a Silicon Micro-strip Tracker (SMT), a Central Fibre Tracker (CFT) and a 2 Tesla superconducting magnet. Preshower detectors have been installed to distinguish between photons and neutral pions. The gaseous muon chambers in the forward and backward regions are replaced with smaller drift tubes having shorter drift time covering a larger region around the interaction point. In Tevatron Run II, $p \bar{p}$ collisions occur at a much higher rate compared to Run I. Thus faster electronics as well as a faster trigger system have been implemented to make fast trigger decisions and to read out the events quickly. In summary, D $\varnothing$ detector assembly contains: (1) Luminosity Detector, (2) Central Tracking System, (3) Preshower Detectors, (4) Calorimeter, and (5) Muon Spectrometer. Brief overview of these components are provided in remaining sections of this chapter. Fig. 2.6 shows a cross-sectional view of the D $\varnothing$ detector.

\subsubsection{Coordinate System}

Positions of objects within the detector, are described with respect to a right-handed coordinate system, with the origin at the centre of the detector, positive $z$-axis along the proton direction and $y$-axis pointing upward. The 


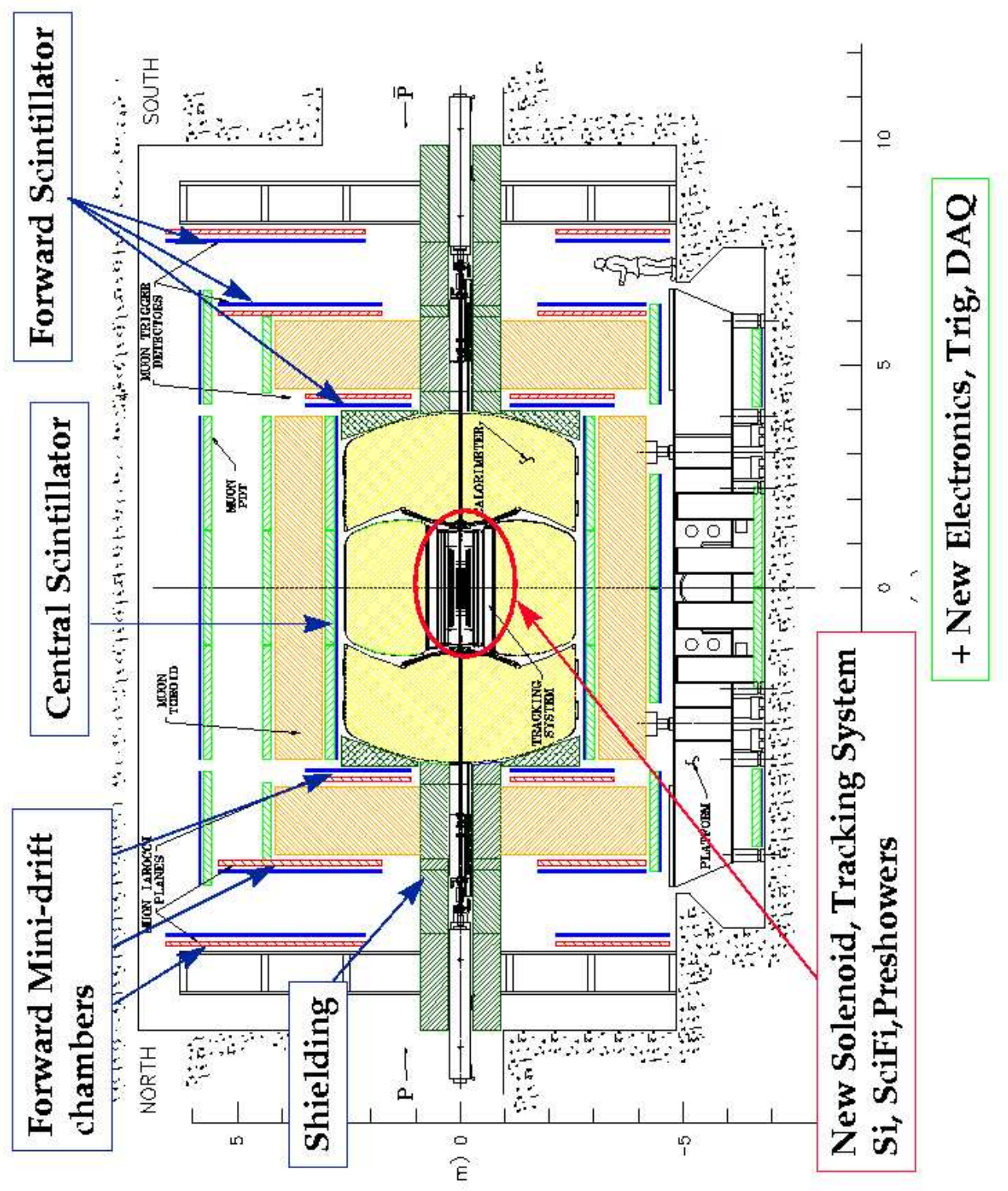

Figure 2.6: A cross-sectional view of the $\mathrm{D} \emptyset$ detector, showing different components. 
radial distance $(\mathrm{r})$ and the azimuthal angle $(\phi)$ in the transverse plane $(\mathrm{x}, \mathrm{y})$ are given by,

$$
\begin{array}{r}
r=\sqrt{x^{2}+y^{2}} \\
\phi=\tan ^{-1} \frac{y}{x}
\end{array}
$$

Instead of using polar angle $\theta$ (defined by the angle with respect to $z$-axis) an convenient variable, called pseudo-rapidity is defined as

$$
\eta=-\ln \tan (\theta / 2)
$$

In ultra-relativistic limit i.e., $m / E \rightarrow 0, \eta$ approaches the true rapidity,

$$
y=\frac{1}{2} \ln \left(\frac{E+p_{z}}{E-p_{z}}\right)
$$

The pseudo-rapidity variable is often used in hadron colliders as the multiplicity distribution of the particles in high energy collisions $(d N / d \eta)$ is roughly constant in $\eta$. Moreover, the pseudo-rapidity interval, $\Delta \eta$ is invariant under Lorentz boost. Fig. 2.7 shows the coordinate system described above. 


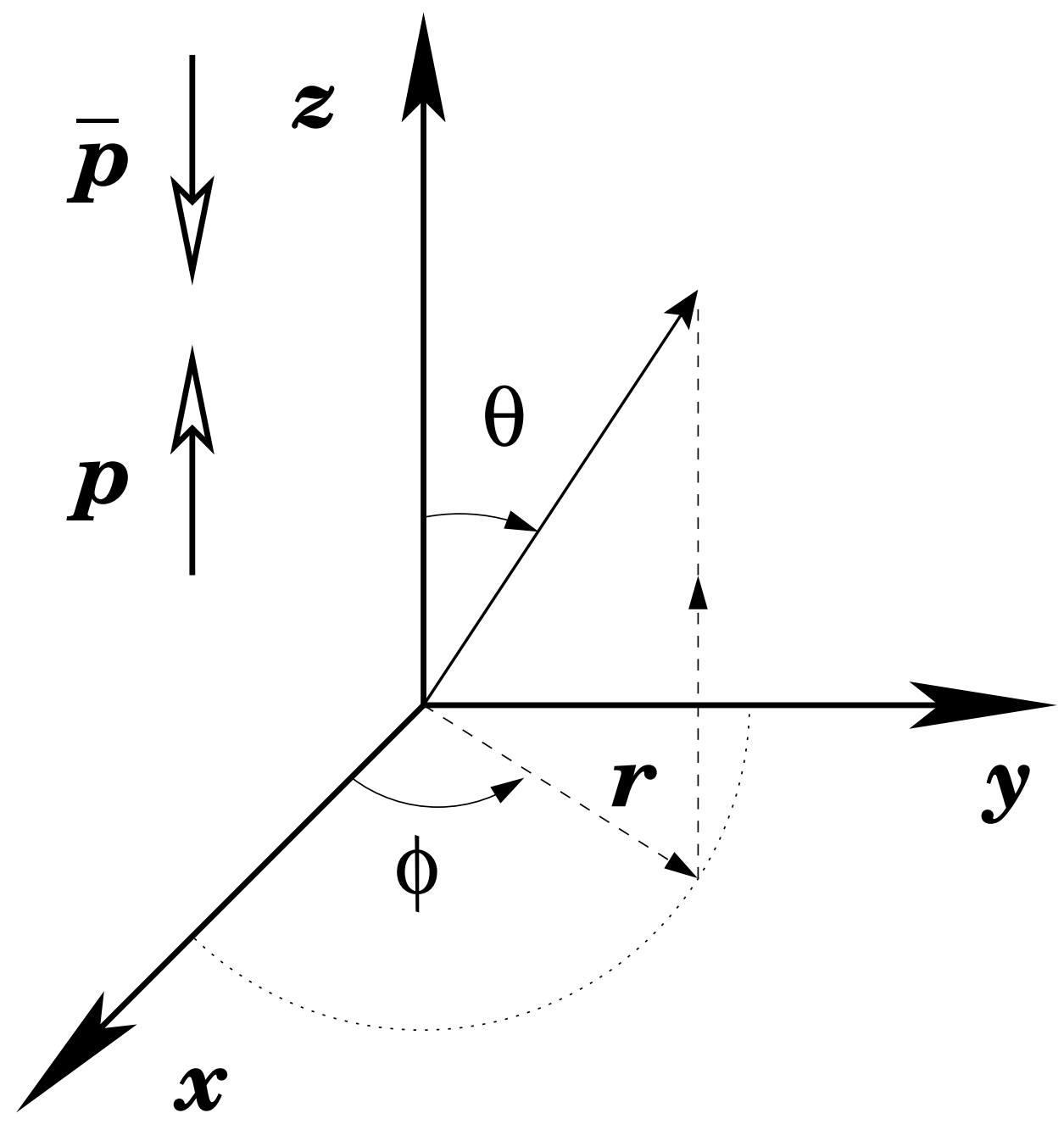

Figure 2.7: The coordinate systems showing the polar angle $(\theta)$, radial distance $(\mathrm{r})$ and azimuthal angle $(\phi)$. 


\subsubsection{Luminosity Monitors}

The primary purpose of the $\mathrm{D} \varnothing$ luminosity monitors is to make an accurate measurement of the beam luminosity. In addition, it is also used in the trigger system to flag a beam crossing and to diagnose the accelerator performance viz., halo rates, size of beam in $x-y$ plane. $\mathrm{\varnothing} \varnothing$ luminosity counters are composed of two sets of scintillation counters, located at $z \approx \pm 1.4$ metres (called south and north counters respectively) surrounding the beam pipe, covering the region $2.7<|\eta|<4.4$ (see Fig. 2.8). Each luminosity monitor is a combination of 24 wedge-shaped $1.6 \mathrm{~cm}$ thick scintillation tiles which are read out by high gain photomultiplier tubes (PMT's) attached to each of them.

In inelastic collision between protons and antiprotons, sprays of particles are produced moving in both directions along the beam axis, which are detected by the luminosity counters. The luminosity monitors use the time difference between the fast hits in north and south counters to distinguish real collisions from the $p / \bar{p}$ halos. Halo particles from proton beam, hit the south counter $\approx 9$ ns after hitting the north counter, while for antiproton halo, this sequence is reversed. The particles produced in $p \bar{p}$ collisions, hit south and north counters almost at the same time $(\approx 4.67$ ns after the 


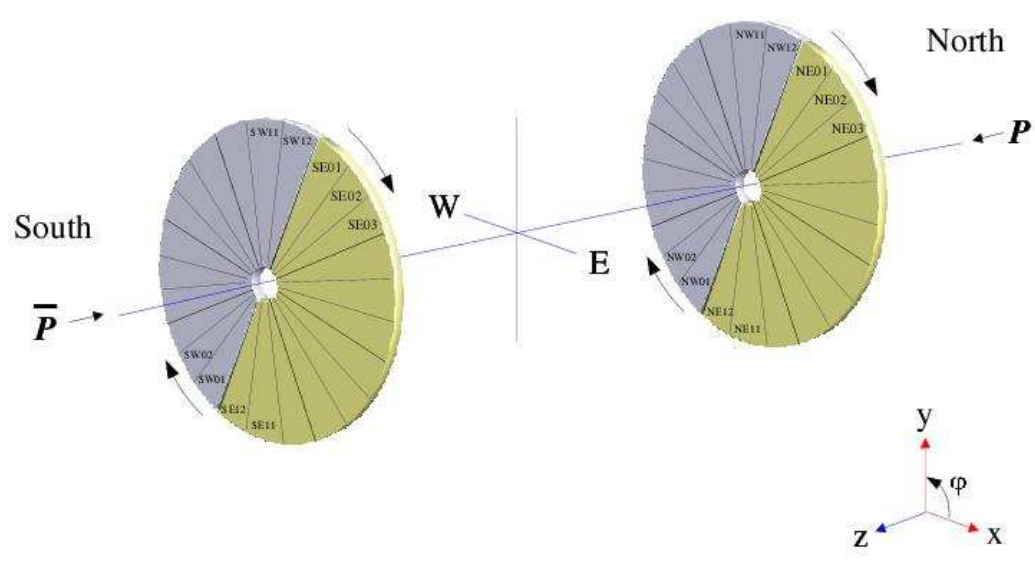

(a)

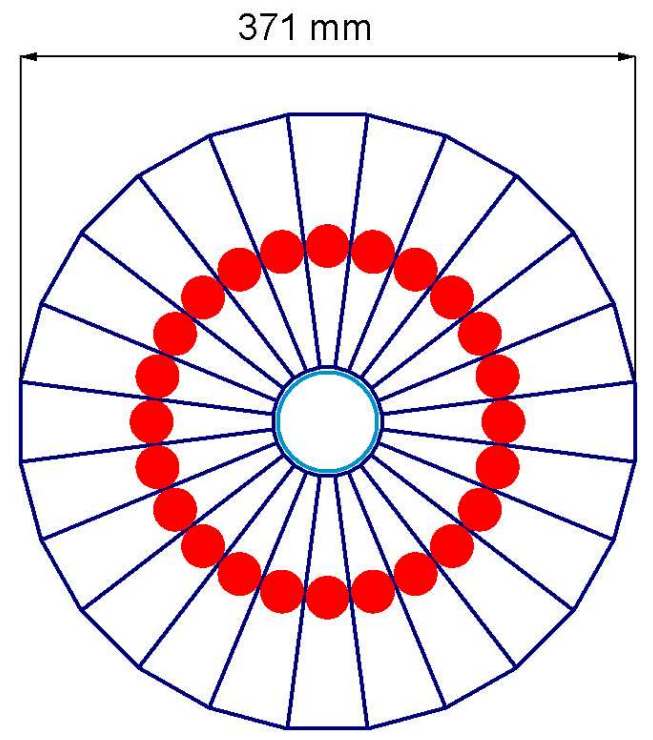

(b)

Figure 2.8: Schematic diagram of DØ luminosity monitors: (a) 3-D layout of two sets of counters, (b) cross sectional view of each monitor (circular spots on each wedge are the places where the PMT's are mounted). 


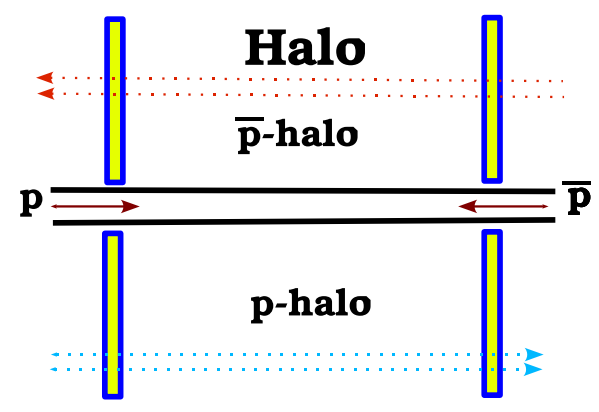

North
South

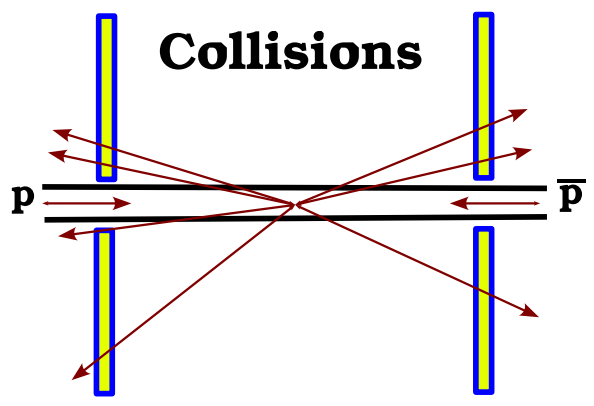

North
South

(a)

Figure 2.9: Cartoon diagram showing the particles from (a) proton/antiproton halos, (b) $p \bar{p}$ collision.

collision). These are pictorially shown in Fig. 2.9. The luminosity counter measures the time-of-flight with an approximate resolution of $0.3 \mathrm{~ns}$. Using the time-coincidence between north and south counters, beam luminosity is estimated, as described in Sec. 4.1.1. The accuracy on the DØ luminosity measurement is $6.5 \%$ [37].

\subsubsection{Central Tracking System}

The central tracking system measures the momentum of the trajectories of charge particles. The combination of Silicon Microstrip tracker (SMT) surrounding the beam pipe, and Central Fibre Tracker (CFT) occupying the outer volume of the central tracking system, provides an excellent position 
resolution for the $\mathrm{D} \varnothing$ tracking system while the solenoidal magnetic field helps to measure the momenta of the charged particles to a great accuracy. Because of its excellent position resolution, the central tracking system is capable of resolving the detached secondary vertices ${ }^{\dagger}$ from the interaction vertex.

SMT and CFT are contained within a cylindrical superconducting magnet of 2.8 metre length, as shown in Fig. 2.10. The superconducting magnet produces a field of 2 Tesla in the direction parallel to the beam and thereby forces the charged particles coming from $p \bar{p}$ collisions, to move in helical trajectories. The central tracking system covers the region of $|\eta|<3.0$.

\section{Silicon Microstrip Tracker}

When a charge particle passes though the wafers of $n$-type silicon in each layer of the Silicon Microstrip Tracker (SMT) is made, it produces pairs of electrons and holes. These charges are collected by aluminium strips attached to $p^{+}$or $n^{+}$type silicon strips, which are attached to the two sides of the silicon wafer. The accumulated charge is proportional to the energy deposited

\footnotetext{
${ }^{\dagger}$ Most of the hadrons containing b-quarks, decay inside the central tracking system after traversing few millimetres from the interaction vertex, where they are produced. Thus the event can have two or more well separated vertices and the vertices associated with the said decays of hadrons containing b-quarks are called secondary vertices.
} 


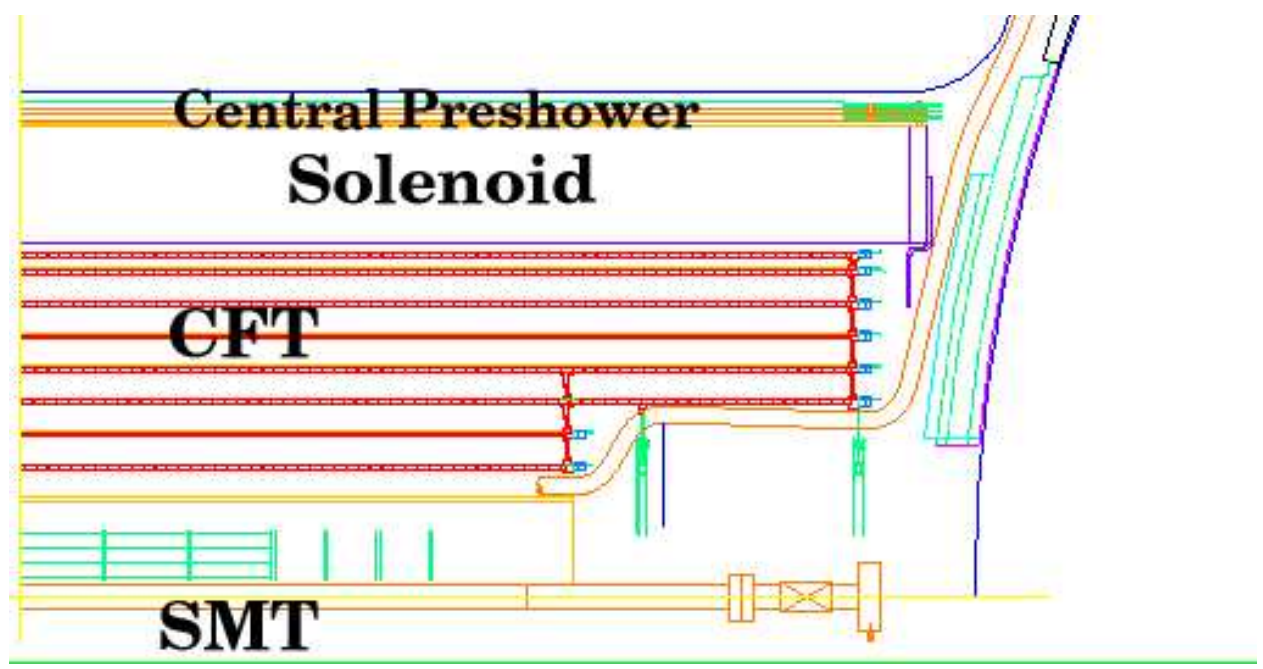

Figure 2.10: Schematic diagram showing the layout of the DØ Central Tracking System.

by the particle in the wafer.

SMT consists of barrel and disk modules as shown in Fig 2.11 and covers the extended interaction region of the colliding beams in z-direction. It serves the purpose of three dimensional tracking and is able to resolve the vertices with an approximate resolution of 30 micron in $x-y$ plane. The barrel detectors measure the coordinates of a charged particle in $r-\phi$ plane, while the disk geometry provide measurements in both $r-z$ and $r-\phi$ planes.

The barrel geometry consists of six cylindrical segments (each $12.4 \mathrm{~cm}$ long), surrounding the beam pipe. Each barrel module contains four con- 

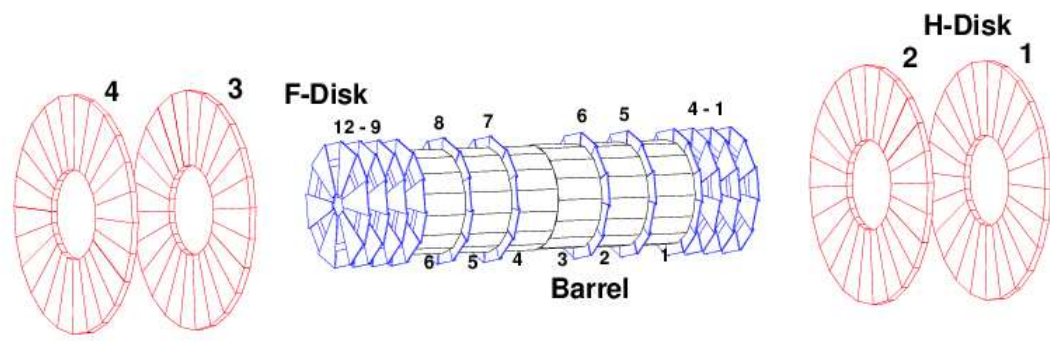

Figure 2.11: Layout showing different parts of the Silicon Microstrip Tracker.

centric layers (L1, L2, L3 and L4 in the order of increasing radius) of silicon ladders with radii ranging from $2.6 \mathrm{~cm}$ to $10 \mathrm{~cm}$. The ladder, which is the basic detector unit, consists of two $300 \mu \mathrm{m}$ thick wafers, of $6 \mathrm{~cm} \times 2.1 \mathrm{~cm}$ cross section, positioned end to end and electrically connected by micro-wire bonds. Layers L2 and L4 are double-sided with $50 \mu \mathrm{m}$ pitch strip on p-side while the n-side has $62.5 \mu \mathrm{m}$ pitch strip at $2^{0}$ stereo angle relative to the beam axis. Four central barrel segments include $90^{\circ}$ stereo double-sided detectors in layers L1 and L3, with $50 \mu \mathrm{m}$ and $156 \mu \mathrm{m}$ pitch strips on the $\mathrm{p}$ and n-sides respectively. Two outer barrels have single-sided $50 \mu \mathrm{m}$ pitch strips in layers L1 and L3. The barrel geometry provides almost hermetic $\phi$ coverage in the central region. Fig. 2.12 is the end view along the beam axis, showing different layers of SMT barrels.

As shown in Fig. 2.11, the disk geometry consist of 12 small disks called 


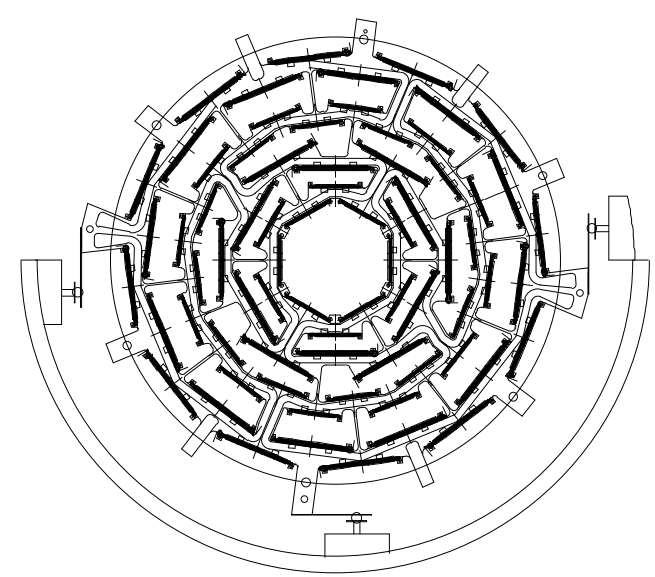

Figure 2.12: End view of the barrel structure in SMT.

F-disks and 4 large disks, called H-disks. Four of the F-disks are interleaved with the barrel modules, while rest of the F disks are situated symmetrically at $|z|=38 \mathrm{~cm}, 43 \mathrm{~cm}, 48 \mathrm{~cm}$ and $53 \mathrm{~cm}$, on both sides of the detector centre. The F-disks are double-sided disks with $\pm 15^{0}$ stereo angles. Each F-disk is a combination of $12 r-\phi$ wedges of $8 \mathrm{~mm}$ thickness. These wedges have slight overlap at the edges to have good coverage. Fig. 2.13 shows $r-\phi$ view of one such F-disk.

Similar to F-disks, H-disks are positioned symmetrically at $|z|=100$ $\mathrm{cm}$ and $121 \mathrm{~cm}$, from the detector centre. A H-disk is an assembly of 24 single-sided wedge shaped detectors, where the Silicon strips in two disks on either side of the detector centre, are at $\pm 7.5^{0}$ stereo angles. Geometrical 


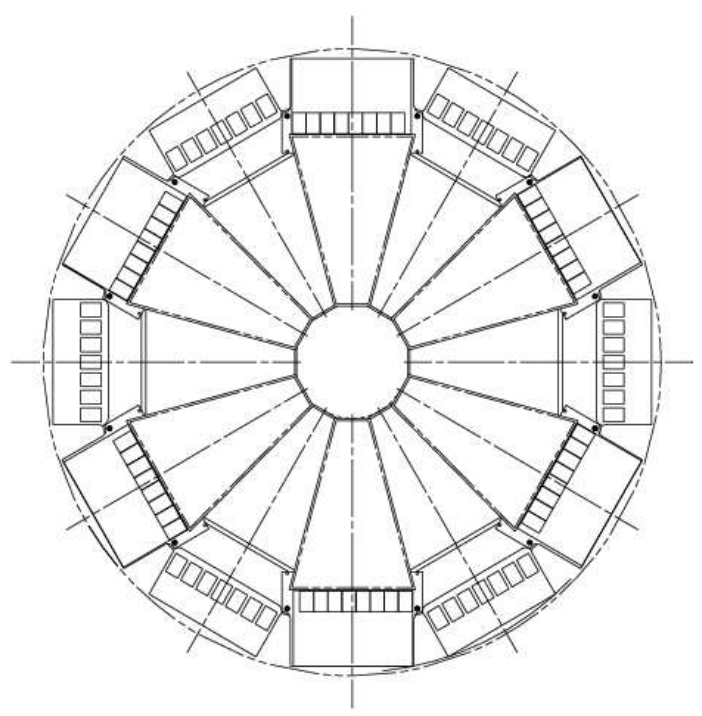

Figure 2.13: $r-\phi$ view of an F-disk in SMT, showing the wedges of silicon detectors.

parameters of different components of SMT are summarized in Tab. 2.1.

SMT has approximately 793,000 signal channels which are read out by SVXIIe chips. When an interaction takes place, the SVXIIe chips accumulate the charges into an array of 32 capacitors for each individual channel. If

\begin{tabular}{l|c|c|c}
\hline Component & Barrel & F-disk & H-disk \\
\hline Layer/Planes & 4 & 12 & 4 \\
Readout length & $12.4 \mathrm{~cm}$ & $7.5 \mathrm{~cm}$ & $14.6 \mathrm{~cm}$ \\
Inner radius & $2.7 \mathrm{~cm}$ & $2.6 \mathrm{~cm}$ & $9.5 \mathrm{~cm}$ \\
Outer radius & $9.4 \mathrm{~cm}$ & $10.5 \mathrm{~cm}$ & $26 \mathrm{~cm}$ \\
\hline
\end{tabular}

Table 2.1: Geometrical parameters of different SMT components. 
the event is triggered, the charges are digitized and passed on to the data acquisition system. Further details regarding SMT can be found in Ref.[38, $39]$.

\section{Central Fibre Tracker}

The Central Fibre Tracker (CFT) is the outer tracker, surrounding the SMT in the central part of DØ detector. CFT consists of 8 cylindrical super-layers (named as A, B, C, D, E, F, G and $\mathrm{H}$ in the order of increasing radius). The CFT covers up to $|\eta|<2.0$. The concentric super-layers of CFT are arranged between radii of $19.5 \mathrm{~cm}$ and $51.5 \mathrm{~cm}$ around the beam pipe. Two inner super-layers situated at radii $20 \mathrm{~cm}$ and $25 \mathrm{~cm}$, are $166 \mathrm{~cm}$ long and rest of the super-layers are $252 \mathrm{~cm}$ long in z direction.

The CFT super-layers are made of closely packed 256 channel fibre ribbons, mounted on Carbon-fibre cylinders. The fibre ribbons are made of $835 \mu \mathrm{m}$ diameter polystyrene wave-length shifting (WLS) scintillating fibres. Each CFT super-layer is a "doublet", containing two sub-layers. Inside each super-layer, the fibres in the inner layer are oriented along the beam axis providing axial measurement of the track position, while the fibres in the outer layers are oriented at a stereo angle of $\pm 3^{0}$ (called $\mathrm{U}$ and $\mathrm{V}$ respectively) with respect to the beam axis. Thus each doublet is capable of stereo 


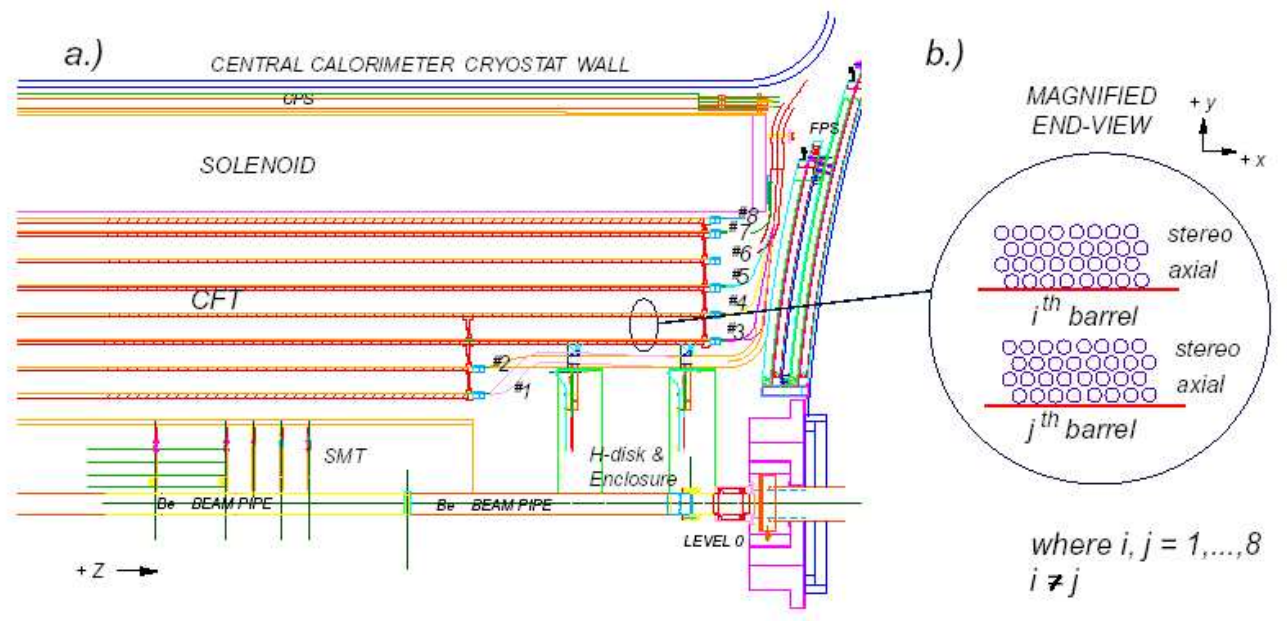

Figure 2.14: Diagram showing the geometry of CFT: a) Cut view of showing a quarter of the detector in $r-z$ with 8 super-layers b) Cross sectional view of the fibres within a ribbon in $r-\phi$.

measurement i.e., $\eta-\phi-z$ coordinates of the track position. Fig. 2.14 shows the construction of CFT layers, while the geometrical parameters are summarised in Tab. 2.2.

Each WLS fibre is optically connected to a clear fibre waveguide ranging from 8-10 $\mathrm{m}$ in length, which transfers the light pulse to a Visible Light Photon Counter (VLPC) photo-sensor. The VLPC is a descendant of the Solid State Photomultiplier, an impurity band silicon avalanche photo-detector, operated at cryogenic temperatures $\left(7-10^{0} \mathrm{~K}\right)$ and is capable of detecting a single photon with high efficiency $(>70 \%)$. Each VLPC has 8 pixels (each of $1 \mathrm{~mm}$ diameter) which receive the signal from clear fibres while a cassette 


\begin{tabular}{|l|c|c|c|}
\hline Layer & Radius $(\mathrm{cm})$ & Fibres/layer & Active length in z-direction $(\mathrm{m})$ \\
\hline A & 19.99 & 1280 & 1.66 \\
AU & 20.15 & 1280 & 1.66 \\
B & 24.90 & 1600 & 1.66 \\
BV & 25.60 & 1600 & 1.66 \\
C & 29.80 & 1920 & 2.52 \\
CU & 29.97 & 1920 & 2.52 \\
D & 34.71 & 2240 & 2.52 \\
DV & 34.87 & 2240 & 2.52 \\
E & 39.62 & 2560 & 2.52 \\
EU & 39.78 & 2560 & 2.52 \\
F & 44.53 & 2880 & 2.52 \\
FV & 44.69 & 2880 & 2.52 \\
G & 49.43 & 3200 & 2.52 \\
GU & 49.59 & 3200 & 2.52 \\
H & 51.43 & 3520 & 2.52 \\
HV & 51.59 & 3520 & 2.52 \\
\hline
\end{tabular}

Table 2.2: Physical parameters of CFT layers. U and V layers are at stereo angles of $+3^{0}$ and $-3^{0}$ respectively. 
of VLPC contains 1024 readout channels. VLPC's convert the light yields into analog electrical signals which are digitized using SVXII chips. Details regarding the DØ CFT and VLPC's can be obtained in Ref. [40].

\section{Performance of Central Tracking System}

Charged particle trajectories are helical due to the solenoidal magnetic field inside the central tracker. The hits from SMT and CFT detectors are utilized to reconstruct the tracks as described in Sec. 3.1.1 later. In principle, tracks originating from the $p \bar{p}$ collision should point to the geometric location of the collision. Therefore, the impact parameter of a track which is the perpendicular distance of the track from the $p \bar{p}$ collision point, is a measure of the tracker performance. The impact parameter (denoted as $\mathrm{d}_{0}$ ) resolution of the Central Tracking system i.e., SMT and CFT combined, is shown in Fig. 2.15 [41] and parametrized as

$$
\sigma\left(\mathrm{d}_{0}\right)=\mathrm{a}_{0}+\frac{\mathrm{a}_{1}}{\mathrm{p}_{\mathrm{T}}}
$$

where

$$
\begin{aligned}
& \mathrm{a}_{0}=8.5 \mu \mathrm{m}, \quad \mathrm{a}_{1}=37.0 \mu \mathrm{m} \mathrm{GeV} \quad \text { (Monte Carlo), } \\
& \mathrm{a}_{0}=11.2 \mu \mathrm{m}, \quad \mathrm{a}_{1}=41.8 \mu \mathrm{m} \mathrm{GeV} \quad \text { (Data). }
\end{aligned}
$$




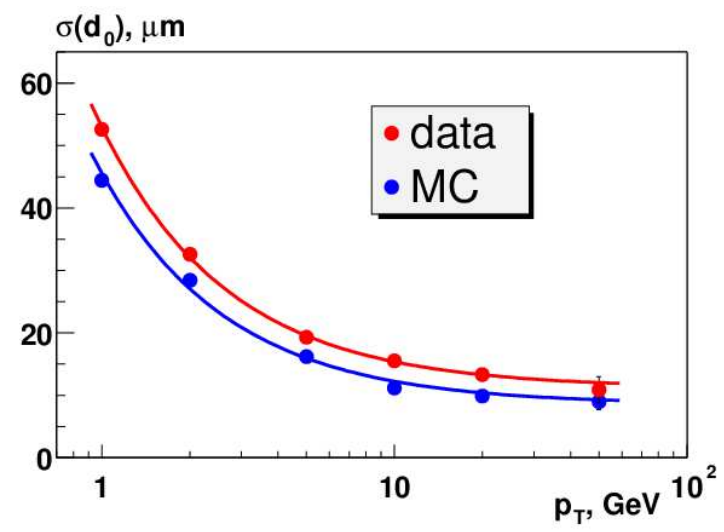

Figure 2.15: Impact parameter resolution, $\sigma\left(d_{0}\right)$ of the Central Tracker as a function of track $p_{T}$.

\subsubsection{Central Preshower Detector}

The Central Preshower Detector (CPS) is designed to improve the electron identification and to restore the electron/photon energy resolution degraded by the presence of the solenoid. The fine segmentation of the CPS detector also helps to distinguish between photons and neutral pions. It is a scintillator based detector, installed in the cylindrical space between the outer edge of the solenoid and the inner face of the Central Calorimeter cryostat, as shown in Fig. 2.16.

The CPS covers a region $|\eta|<1.2$. There are three concentric layers of 

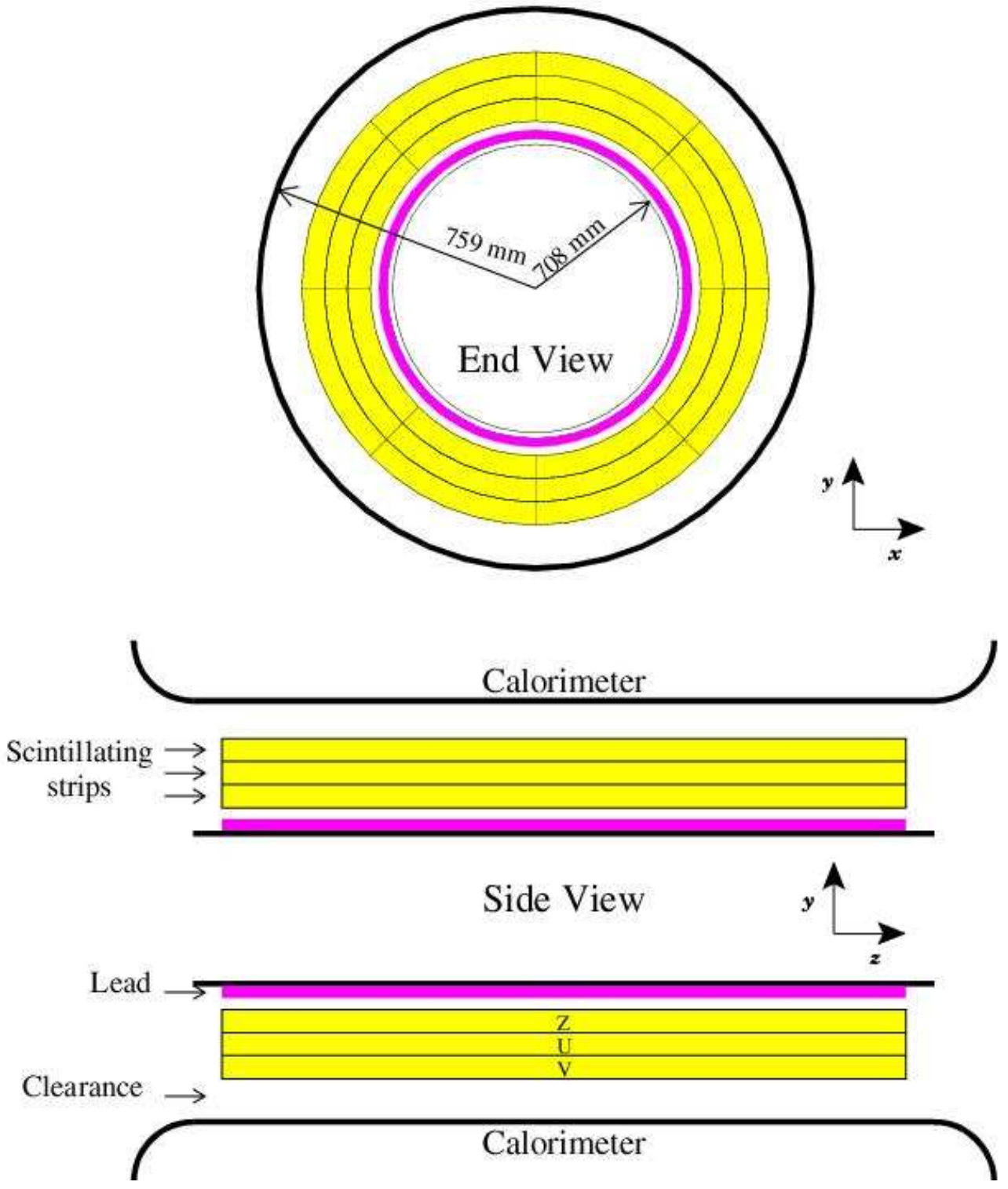

Figure 2.16: Schematic side and end views of DØ Central Preshower detector. 
scintillator strips, the innermost of which is oriented axially in z-direction, while outer two layers are placed at $\pm 22.5^{0}$ stereo angles (named $\mathrm{U}$ and $\mathrm{V}$ respectively). The plastic scintillator strips are equilateral triangles in cross section, with a $1 \mathrm{~mm}$ diameter hole at the centre containing a wavelength shifting fibre. To facilitate detector construction, each layer is made in 8 octants. The WLS fibres are split at $\mathrm{z}=0$ and are connected to the clear fibres at both ends. The triangular strips are arranged in an interlocking saw tooth manner as shown in Fig. 2.17. A lead absorber plate, tapered in z (shown in Fig. 2.16) is placed in front of the scintillator strips in order to present a uniform two radiation length (see the definition of radiation length in the calorimeter section, denoted as $X_{0}$ ) thickness in combination with the solenoid, over the entire $\eta$ range. The CPS channels are read out by the VLPC's, as done for CFT. A detailed design report of CPS can be found in Ref. [42].

\subsubsection{Forward Preshower Detectors}

The Forward Preshower Detectors (FPS) are two identical detectors mounted on the inner faces of end cap calorimeter cryostats and cover a pseudorapidity region of $1.4<|\eta|<2.5$ [43]. The design of the FPS is similar 


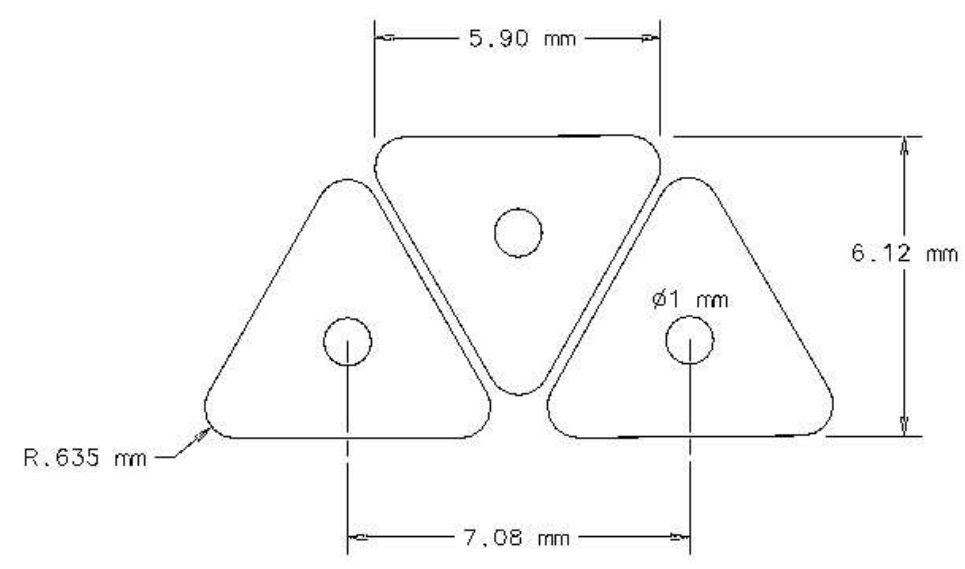

Figure 2.17: The cross section of a layer of the CPS, showing the dimension of each unit. The triangles are made of extruded plastic scintillator and the circles in the middle contain WLS fibres. Each triangular strip is wrapped in reflective material to increase light yield and reduce cross talk.

to that of CPS. There are four layers in FPS, inner $\mathrm{U}$ and $\mathrm{V}$ and outer $\mathrm{U}$ and $\mathrm{V}$. The FPS is segmented into 16 azimuthal wedges, each of which consists of $\mathrm{U}$ and $\mathrm{V}$ stereo layers. A tapered lead absorber plate is placed between inner and outer layers (see Fig. 2.18 for the geometry) to maintain a thickness of $11 \mathrm{~mm}$ or 2 radiation lengths. The inner layers are designed to detect minimally-ionising particles (MIP), while the outer layers are capable of detecting the starting point of showers (the showers are explained in the calorimeter section). Due to the above reason, the layers are often referred to as MIP and shower layers.

As the solenoid does not shadow the forward calorimeters along the 
trajectories from the interaction point, the FPS is designed to discriminate between photons and electrons ${ }^{\dagger}$. If a particle is not observed in the MIP layer, but has a shower in the outer layer, it is likely to be a photon as it does not interact with MIP layer, in front of the lead absorber. Electrons $\S$ on the other hand, are expected to produce signals in both MIP and outer layers. As in CPS, the FPS channels also have a readout system containing clear fibres and VLPC's.

\subsubsection{Calorimeter}

Calorimeter measures energies of electrons, photons and hadronic jets over a large region of pseudo-rapidity $(|\eta|<4)$. Calorimeter also distinguishes between electrons/photons and hadronic jets and provides sufficient information to calculate the missing transverse energy associated with non-interacting particles such as neutrinos. An isometric view of the $\mathrm{D} \varnothing$ calorimeter is shown in Fig. 2.19.

When a high energy ( $\gtrsim 10 \mathrm{GeV}$ ) electron passes through a material with high atomic number, the dominant mechanism through which it loses energy

\footnotetext{
${ }^{\dagger}$ It is very difficult to distinguish between electrons and photons using only the calorimeter information, as they generate almost identical showers in the electromagnetic part of the calorimeter.

$\S$ A charged pion can have a signature similar to an electron in the FPS, but has very different characteristics in terms of showering in the calorimeter.
} 


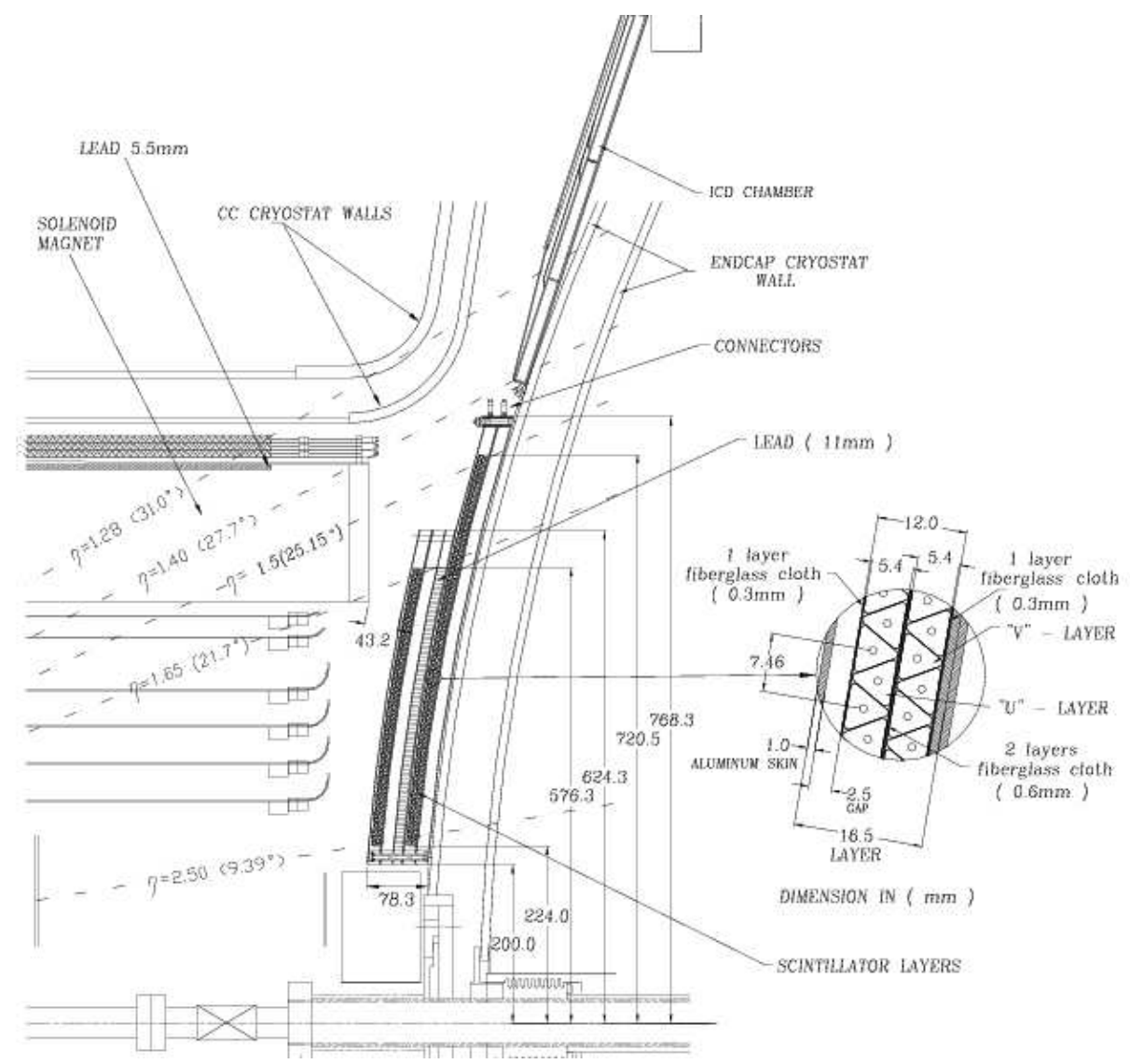

Figure 2.18: Diagram showing a $\mathrm{y}-\mathrm{z}$ view of the FPS detector. 


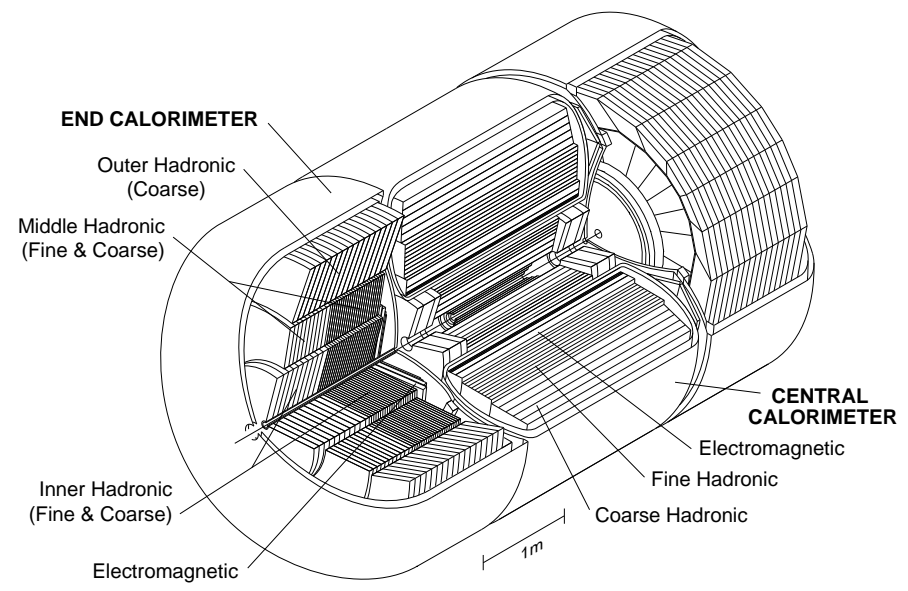

Figure 2.19: An isometric view of the $\mathrm{D} \varnothing$ calorimeter.

is Bremsstrahlung radiation, while a photon loses its energy through electronpositron pair production. The particles emitted in these processes viz., Bremsstrahlung and pair-production, themselves undergo Bremsstrahlung or pair-production. Thus the passage of high energy electrons/photons through a dense material, results in a shower of secondary electrons, positrons and photons, called an electromagnetic shower. If an incident electron/photon loses $\mathrm{dE}$ amount of energy after traversing $\mathrm{dx}$ distance in the material, the energy loss can be expressed as

$$
\frac{\mathrm{dE}}{\mathrm{E}}=-\frac{\mathrm{dx}}{\mathrm{X}_{0}}
$$


where $X_{0}$ is called the radiation length and is the thickness of the material required for the electron/photon energy to become 1/e of the incident energy. It is a constant for a given type of material e.g., $3.2 \mathrm{~mm}$ (for Uranium).

Hadrons interact with the material through a different mechanism and lose energy energy primarily through inelastic collisions with the atomic nuclei. These collisions produce secondary hadrons, which in turn undergo inelastic collisions and thus result in hadronic showers. The process continues until all particles have either been stopped by ionisation losses or absorbed by nuclear processes. The energy loss of the incident hadrons can again be described by the Eqn. 2.5 by replacing $X_{0}$ with $\lambda_{0}$, called the $i n$ teraction length. The interaction length determines the scale for the size of the hadronic showers and is a constant of the material e.g., $10.5 \mathrm{~cm}$ (for Uranium). Thus, hadronic showers are much more extended in space than electromagnetic showers produced by particles of similar incident energy.

DØ calorimeter is a "sampling calorimeter" where layers of inert absorber are interleaved with layers of active material, which is sensitive to the particles passing through it. The layers are usually in the form of planes normal to the direction of the incident particles. The absorber plates help to contain the shower in a manageable volume, but the part of the shower contained in the absorber volume can not be read out. The active mate- 
rial collects the signals created in the absorber volume and sends them to the readout electronics. D $\varnothing$ uses Liquid Argon (LAr) as the active material while layers of depleted Uranium, Copper and Stainless steel (outer calorimeter) are used as absorber materials.

Apriori there is no reason for the response of a calorimeter (i.e., the ratio of the measured signal to the energy of an incident particle) for electromagnetic and hadronic showers to be the same. In fact, the response for hadronic showers tends to be smaller, since $\nu$ 's and $\mu$ 's produced by $\pi$ and $K$ decays, escape detection in the calorimeter. The energy spent in breaking up nuclei would also be invisible. This is quantified by the $e / \pi$ ratio i.e., the ratio of the calorimeter responses to electrons/photons and pions. It is highly desirable that this number be close to 1 , as a hadronic shower may also contain electromagnetic components coming from $\gamma^{\prime}$ s (from $\pi^{0}$ and $\eta$ decays), apart from the hadronic components. The fraction of a hadron's energy which is deposited as electromagnetic showers can undergo large variations from shower to shower, but if the $e / \pi$ ratio is 1 , these fluctuations do not affect the energy resolution. A calorimeter with $e / \pi \approx 1$ is called a compensating calorimeter. The choice of Uranium as the absorber and liquid Argon makes the D $\varnothing$ calorimeter nearly compensating, with $e / \pi=1.11$ at 10 $\mathrm{GeV}$ falling to $e / \pi=1.04$ at $150 \mathrm{GeV}$. 
DØ calorimeter consists of three main sections: the central calorimeter (CC) and two end-cap calorimeters (EC). Each section is divided laterally in three types of modules: the electromagnetic (EM), the fine hadronic $(\mathrm{FH})$ and the coarse hadronic $(\mathrm{CH})$. A module consists of a stack of interleaved absorber plates and signal boards. The spaces between the absorber plates and signal boards, are filled with liquid Argon active medium. The signal board consists of a Copper pad sandwiched between two $0.5 \mathrm{~mm}$ thick pieces of G10 and the outer surfaces of these boards contain a resistive epoxy coating. During operation, the absorber plates are grounded, a positive voltage of $2.0-2.5 \mathrm{kV}$ is applied to the resistive coatings. As a shower develops in the calorimeter, charged particles crossing the LAr gap leave a trail of ionisation. The liberated electrons are collected on the signal board after a drift time of $450 \mathrm{~ns}$. In order to measure the position of showers in transverse direction, the readout pads are subdivided into small cells. A schematic view of one such unit is shown in Fig. 2.20. The cells of adjacent boards are ganged together in depth to form readout cells. For example, 7 layers are ganged together to form the third readout layer of the electromagnetic part of the calorimeter. This ganging varies from module to module as well as on the type of module (see Tab. 2.3 and 2.4).

In both the $\mathrm{CC}$ and $\mathrm{EC}$, the size of a typical readout cell is $0.1 \times 0.1$ 


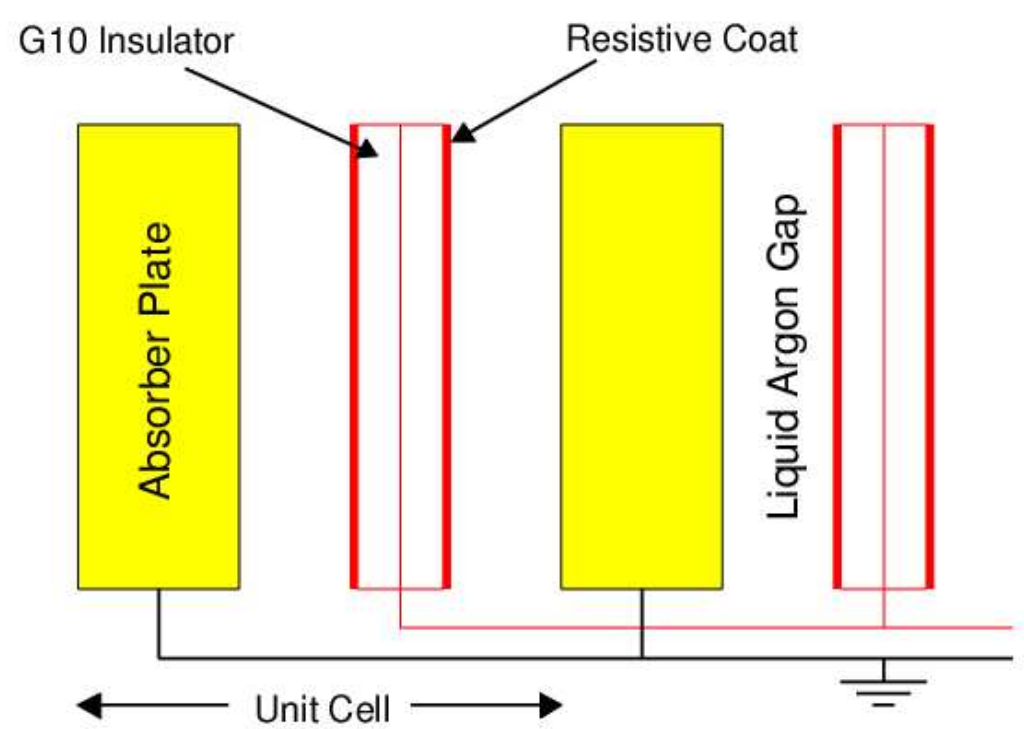

Figure 2.20: Typical unit cell in DØ sampling calorimeter.

in $\eta-\phi$ space. However, in the third layer of the EM modules, where electromagnetic showers typically deposit the bulk of their energy, the readout cells have sizes of $0.05 \times 0.05$. In addition, cells with $|\eta|>3.2$ have a size of $0.2 \times 0.2$ in $\eta-\phi$ space. See Fig. 2.21 for an illustration of the calorimeter segmentation.

\section{Central Calorimeter}

The central calorimeter (CC) provides coverage for $|\eta|<1.0$ and consists of 3 concentric cylindrical shells. These shells are segmented in $\phi$. There are 32 


\begin{tabular}{|lccc|}
\hline Module type & EM & FH & CH \\
\hline Rapidity coverage & \pm 1.2 & \pm 1.0 & \pm 0.6 \\
Number of modules & 32 & 16 & 16 \\
Absorber ${ }^{a}$ & DU & DU-Nb & $\mathrm{Cu}$ \\
Absorber thickness $(\mathrm{mm})$ & 3 & 6 & 46.5 \\
Argon gap (mm) & 2.3 & 2.3 & 2.3 \\
Number of signal boards & 21 & 50 & 9 \\
Number of readout layers & 4 & 3 & 1 \\
Cells per readout layer & $2,2,7,10$ & $20,16,14$ & 9 \\
Total radiation lengths $\left(X_{0}\right)^{b}$ & 20.5 & 96.0 & 32.9 \\
Total nuclear absorption lengths $(\lambda)^{b}$ & 0.76 & 3.2 & 3.2 \\
Sampling fraction $(\%)$ & 11.79 & 6.79 & 1.45 \\
Total readout cells & 10,368 & 3000 & 1224 \\
\hline
\end{tabular}

Table 2.3: Central Calorimeter Parameters.

\footnotetext{
${ }^{a}$ Depleted uranium (DU), depleted uranium with $1.7 \%$ niobium (DU-Nb), or copper $(\mathrm{Cu})$.

${ }^{b}$ At $\eta=0$.
}

identical EM modules in the inner shell, which are thick enough to contain most electromagnetic showers. The next shell is comprised of $16 \mathrm{FH}$ modules, which measure the showers due to the passage of hadrons. The surrounding shell contains $16 \mathrm{CH}$ modules which measure any leakage of energy out of the FH layers. The parameters of CC modules are provided in Table 2.3.

\section{End Calorimeters}

The end calorimeters (EC) provide coverage for $1.0<|\eta|<4.2$ and each contains four module types as shown in Fig. 2.21. The module closest to the central tracker is the EM section. The hadronic sections are arranged in a 


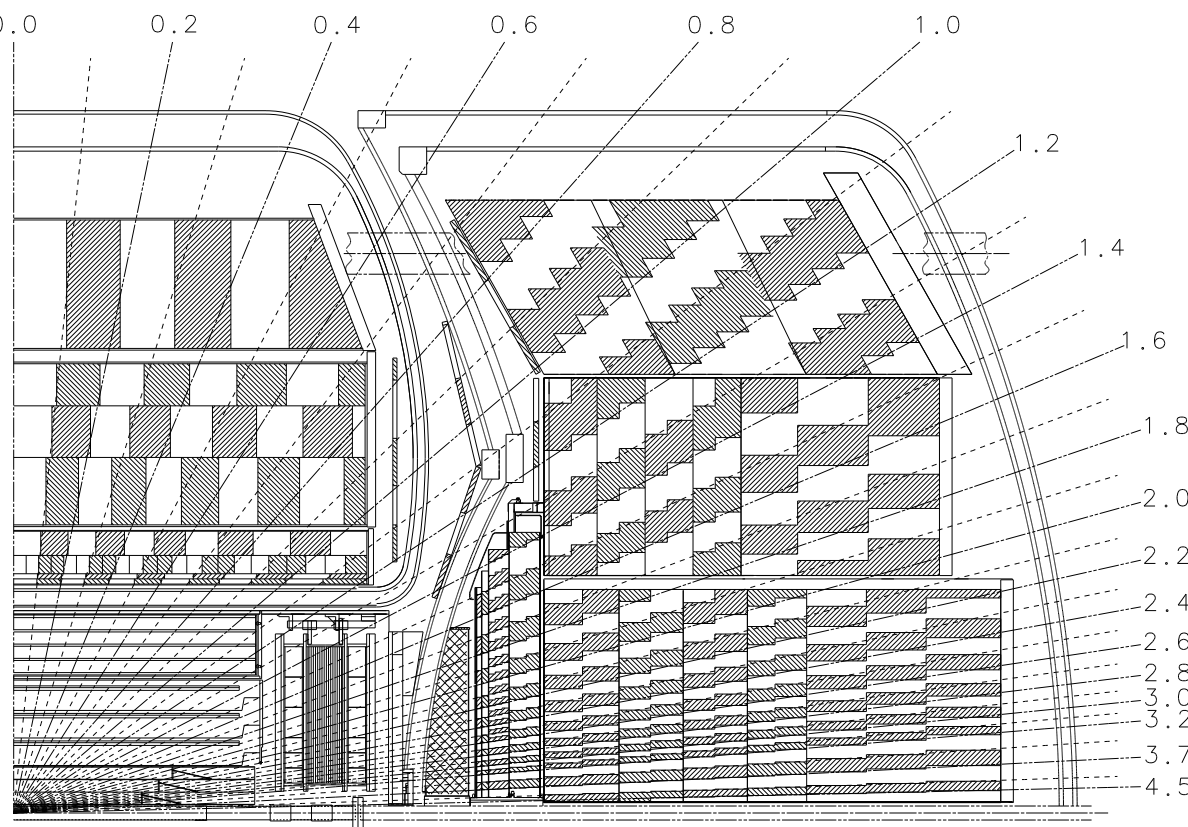

Figure 2.21: One quarter section view of the $D \varnothing$ calorimeters showing the segmentation pattern.

concentric manner, with the module closest to the beam-pipe being called the inner hadronic (IH) modules, surrounding them are the middle hadronic $(\mathrm{MH})$ modules and outer most are the outer hadronic $(\mathrm{OH})$ modules. There are one EM module, one $\mathrm{IH}$ module and 16 concentric rings of $\mathrm{MH}$ and $\mathrm{OH}$ modules in each end calorimeter. As in $\mathrm{CC}$, Inner and Middle hadronic sections are subdivided into two types of layers, $\mathrm{FH}$ and $\mathrm{CH}$, called $\mathrm{IFH}$, $\mathrm{ICH}, \mathrm{MFH}$ and $\mathrm{MCH}$ respectively. Table 2.4 summarises the parameters of EC. 


\begin{tabular}{|lcccccc|}
\hline Module type & EM & IFH & ICH & MFH & MCH & OH \\
\hline Rapidity range & $1.3-3.7$ & $1.6-4.5$ & $2.0-4.5$ & $1.0-1.7$ & $1.3-1.9$ & $0.7-1.4$ \\
No. of modules & 1 & 1 & 1 & 16 & 16 & 16 \\
Absorber ${ }^{a}$ & DU & DU-Nb & SS & DU-Nb & SS & SS \\
Thickness (mm) & 4 & 6 & 6 & 6 & 46.5 & 46.5 \\
LAr gap (mm) & 2.3 & 2.1 & 2.1 & 2.2 & 2.2 & 2.2 \\
Signal boards & 18 & 64 & 12 & 60 & 14 & 24 \\
R.O. layers & 4 & 4 & 1 & 4 & 1 & 3 \\
Cells/r.o. layer & $2,2,6,8$ & 16 & 14 & 15 & 12 & 8 \\
Total rad. len. & 20.5 & 121.8 & 32.8 & 115.5 & 37.9 & 65.1 \\
Total abs. len. & 0.95 & 4.9 & 3.6 & 4.0 & 4.1 & 7.0 \\
Samp. frac. (\%) & 11.9 & 5.7 & 1.5 & 6.7 & 1.6 & 1.6 \\
Tot. r.o. cells & 7488 & 4288 & 928 & 1472 & $384+64+896^{b}$ \\
\hline
\end{tabular}

Table 2.4: End Calorimeter Parameters.

\footnotetext{
${ }^{a}$ Depleted uranium (DU), depleted uranium with $1.7 \%$ niobium (DU-Nb), or stainless steel (SS).

${ }^{b} \mathrm{MCH}$ and $\mathrm{OH}$ cells are summed together at $|\eta|=1.4$
}

\section{Inter Cryostat Detector}

As shown in Fig. 2.21, significant amount of material is located in the transition region between the $\mathrm{CC}$ and the $\mathrm{EC}(0.8<|\eta|<1.4)$. This space is mainly occupied by cryostat walls and support structures for the calorimeter modules. In order to correct for the energies deposited in these un-instrumented regions, scintillator-based counters are placed into these gaps. Two such scintillator arrays, called Inter Cryostat Detectors (ICD) are mounted on the outer surfaces of the EC cryostats. ICD's are supplemented by two detectors called Massless Gap (MG), where signal boards are mounted on the end plates of the $\mathrm{CCFH}, \mathrm{ECMH}$, and ECOH modules. Both 
MG and ICD have the standard segmentation of $0.1 \times 0.1$ in $\eta-\phi$ space.

\section{Calorimeter Readout}

When a particle passes through the calorimeter the drift current between the absorber plate and the resistive surface of the readout cell, induces an image charge on the copper pad located inside the readout cell. The accumulated charge is then integrated and transformed proportionally into voltage pulses by the calorimeter preamplifier. The rise time of of the pulse $(\sim 430 \mathrm{~ns})$ is basically the electron drift time in the LAr gap. The life-time of the pulse decay is $\sim 15 \mu \mathrm{s}$, which is much larger than the bunch crossing time. The signals from the pre-amplifiers are then transferred to the baseline subtracter (BLS) circuits where the preamplifier output is differentiated to extract the height of the voltage pulse. In the BLS circuits, a pedestal value which is an average of cell signals, during the absence of beam in the beam pipe and are measured on a routine basis, is also subtracted from the signal obtained due to the passage of a particle. Next, the analog signal from the BLS is carried to the first two levels of triggering system. If the event satisfies a calorimeter trigger, the analog signal is digitized and passed on to the Data Acquisition System. Detailed description of Run II calorimeter electronics can be found in Ref. [44]. 


\section{Performance of the Calorimeter}

As mentioned above, the final signal is the number of electrons which are registered in the readout pads. Two important measures of performance are the resolution of energy measurement and the linearity of response. The resolution of the calorimeter for measuring the energy of an incident particle is determined by the fluctuations in the number of these electrons. These fluctuations have several sources:

- Sampling fluctuations - fluctuations in the energy deposited in the active layers due to shower development.

- Leakage of energy out of the calorimeter.

- Noise in the active layers due to natural radioactivity of the depleted uranium plates.

- Electronic noise.

- Gain variations - high voltage, electronics, LAr temperature, oxygen contamination in LAr.

The resolution of $\mathrm{D} \varnothing$ calorimeter is parameterized as

$$
\left(\frac{\sigma(E)}{E}\right)^{2}=C^{2}+\frac{S^{2}}{E}+\frac{N^{2}}{E^{2}}
$$


where $\mathrm{C}$ is the constant term which represents calibration errors and energy leakage, $\mathrm{S}$ is the sampling fluctuation term, and $\mathrm{N}$ is the noise term which includes electronics and Uranium noise and contributes to a constant variance in $\sigma_{E}$ independent of the energy. From the test beam data [45], it is found that for electrons, $C=0.003 \pm 0.002, S=0.157 \pm 0.005(G e V)^{\frac{1}{2}}$, and $N \approx$ $0.140 \mathrm{GeV}$. And for pions, $C=0.032 \pm 0.004, S=0.41 \pm 0.04(G e V)^{\frac{1}{2}}$, and $N \approx 1.28 \mathrm{GeV}$. Refs. [46, 47] can be consulted for further details regarding $\mathrm{D} \varnothing$ calorimeter.

\subsubsection{Muon System}

The muon spectrometer is located in the outermost part of the $\mathrm{D} \varnothing$ detector. Muons are primarily identified by their penetrating nature. They do not undergo hadronic interaction but only lose energy through ionisation. Muon system identifies the muons and measures charge and momentum of the muons.

The D $\varnothing$ muon system (Fig. 2.22) consists of three types of detectors viz., Proportional Drift Tubes (PDT), Mini Drift Tubes (MDT) and Scintillation counters [48], arranged in three layers called A, B and C. The system is subdivided into three subsystems, the Wide Angle Muon System (WAMUS) 
or Central Muon System and two Forward Angle Muon Systems (FAMUS) or Forward Muon Systems. A 2 Tesla Toroid magnet is used in the muon system for the measurement of muon momenta by deflecting the muon trajectories in $r-z$ plane. The innermost layer located between the calorimeter and the iron of the toroid magnet is called A layer. B and C layers are located outside the iron. The region, underneath the cryostats containing the calorimeter, only have partial coverage with muon detectors, as the region contains support structures for the $\mathrm{D} \varnothing$ detector and readout electronics.

\section{Central Muon System}

The Central Muon System or WAMUS has a coverage of $|\eta|<1$., designed with PDT's and scintillation counters. The PDTs used in WAMUS are rectangular in cross section, with one sense wire per drift cell. They are formed out of aluminum extrusions into rectangular modules containing either four (A layer) or three (B and C layers) planes of drift tubes (see Fig. 2.23). The sense wires (50 $\mu \mathrm{m}$ Gold-plated Tungsten inside the tubes) are oriented parallel to the direction of the magnetic field in the iron toroid, surrounded by a gaseous mixture of $80 \%$ Argon, $10 \% \mathrm{CF}_{4}$ and $10 \% \mathrm{CH}_{4}$. Ionisation created by the passage of a muon though the drift cells are collected by the sense wires and the drift time is the measure for the deflection due to the magnetic 


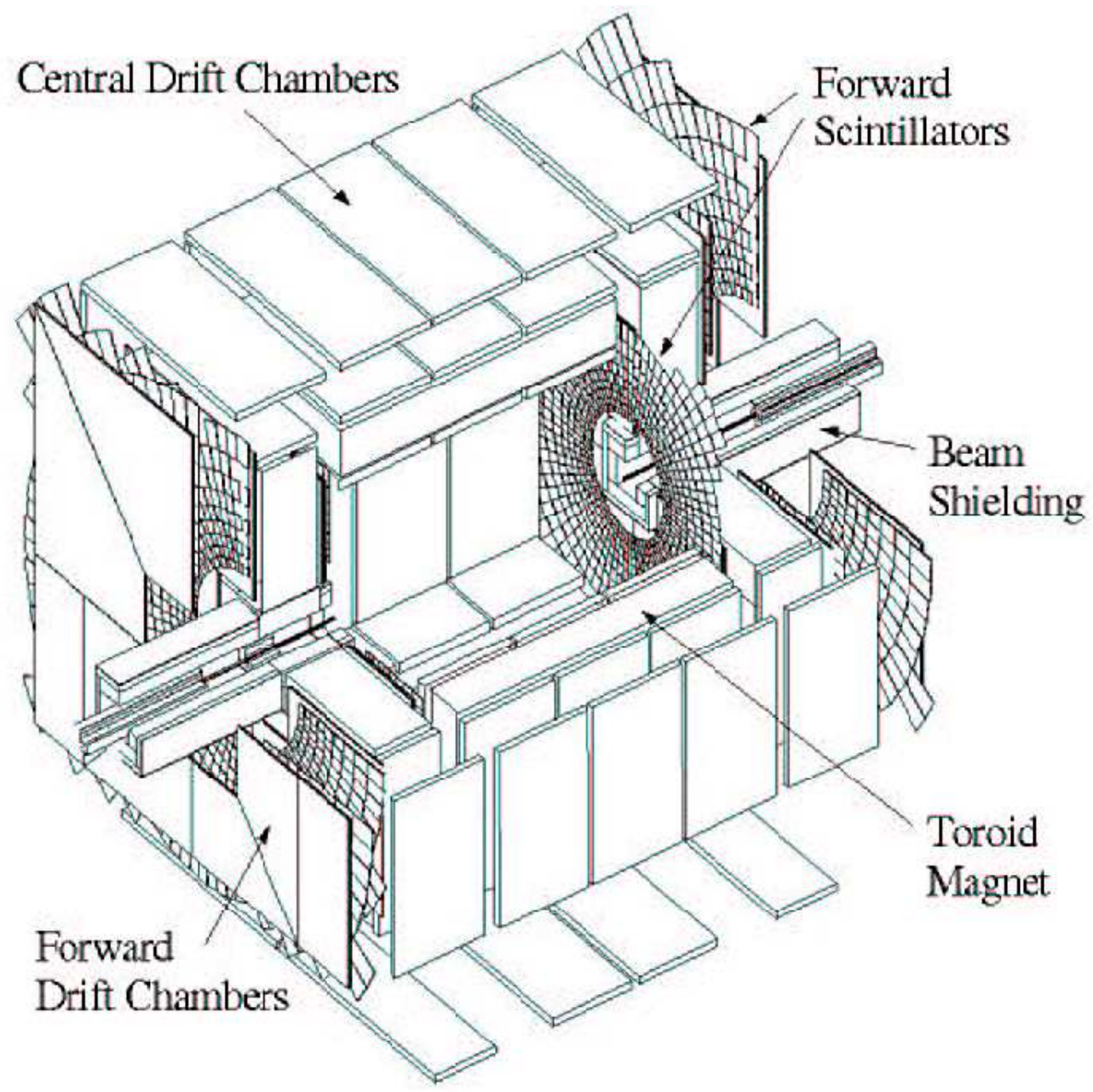

Figure 2.22: A cut-away view of the D $\varnothing$ Muon system. 


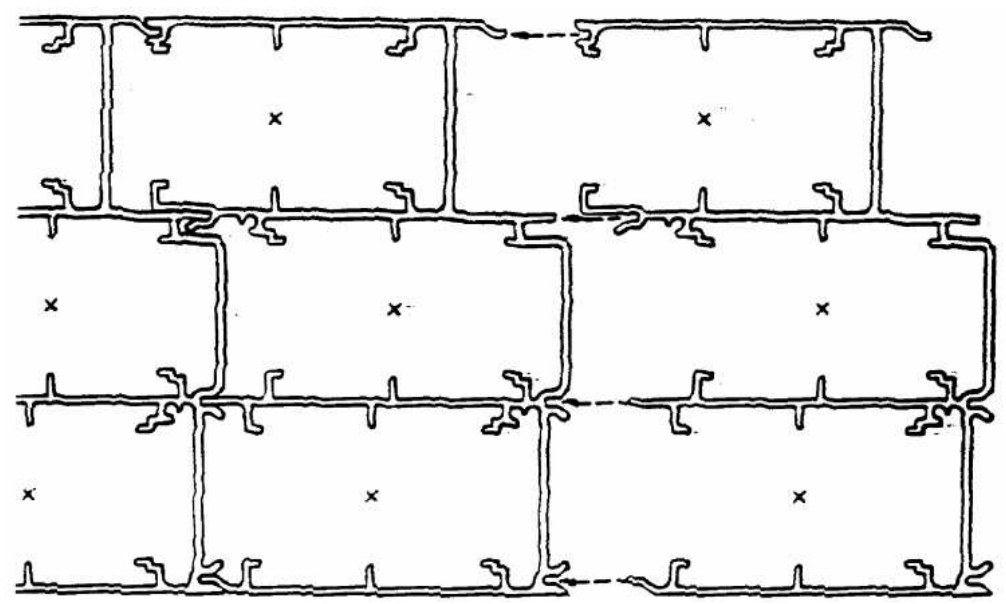

Figure 2.23: Extruded aluminum sections from which the B and C layer PDT chambers are constructed. The A layer chamber extrusions are similar, but have four cells instead of three. The 'x' marks the position of the sense wire.

field. The distance of a hit from the sense wire can be determined with a resolution of about $500 \mu$.

In order to facilitate access to the chamber electronics, all readout is done from one end of the drift tubes. To accomplish this, tubes are jumpered together in pairs on one end. The front end electronics of PDT measures the arrival times of pulses at the end of each wire, as well as the time difference between pulses arriving at the ends of each jumpered pair of sense wires. This time difference is a crude measure of the position of a hit along the sense wire with resolution of $10-20 \mathrm{~cm}$. More precise determination of the hit position is performed by inserting two vernier cathode pads at the top and bottom 


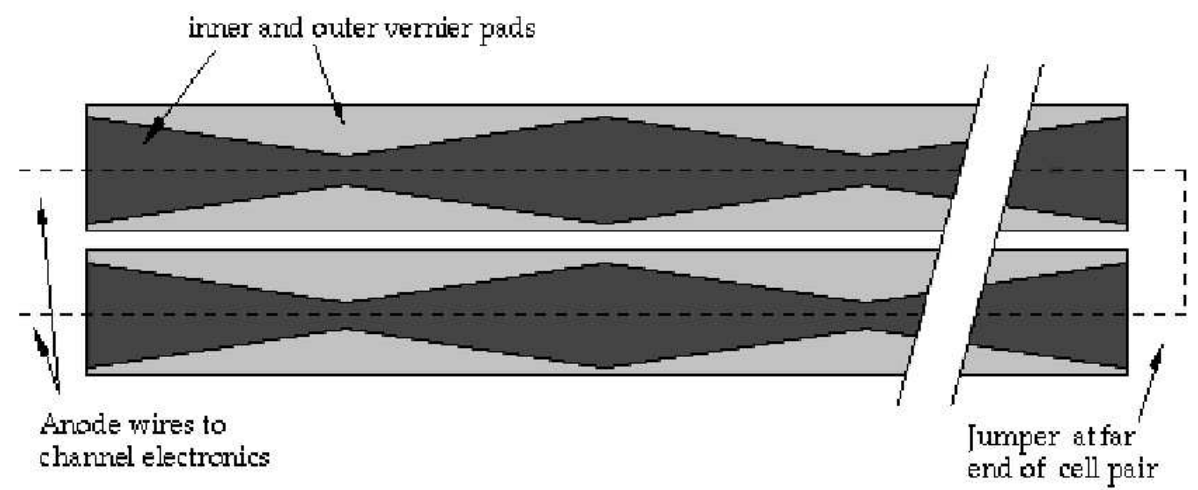

Figure 2.24: WAMUS pads viewed from the top.

of each tube (see Fig. 2.24). These pads forming a diamond pattern which repeats with a period of $\approx 60 \mathrm{~cm}$, are separated into inner and outer regions. Electron avalanches on the sense wires induce pulses on the cathode pads while the ratio of the charges deposited on the inner and outer pads can be used to localize the hit with a resolution of about $5 \mathrm{~mm}$ [49].

The A layer of Central Muon system contains a layer of scintillation counters on the calorimeter side, called $A \phi$ counters. The $\phi$ segmentation of the layer is $\approx 4.5^{0}$, while the layer is longitudinally divided into 33 segments, each of $\approx 0.64 \mathrm{~cm}$ length. This segmentation provides additional position measurement along the orientation of the PDT sense wires, while making precise measurement of muon arrival time. It is used as a fast trigger signal for PDT's. The C layer of the central muon system also contains a layer 
of scintillation counters which are used to distinguish between the cosmic muons and the muons coming from $p \bar{p}$ collisions.

\section{Forward Muon System}

The extended coverage for muon detection up to $|\eta|<2$ is achieved by designing the Forward Muon Systems. They consist of MDT's and pixel scintillation counters, with A layers in between the End Cap Calorimeters and toroids, while the $\mathrm{B}$ and $\mathrm{C}$ layers are located outside the toroids. As in Central Muon System, there are 4 planes of MDT's in A layers while B and C layers contain 3 planes each. Each MDT unit consists of 8 cells, each with a $9.4 \mathrm{~mm} \times 9.4 \mathrm{~mm}$ cross section and a $50 \mu \mathrm{m}(\mathrm{W}-\mathrm{Au})$ anode wire at the centre. The gas used for the tubes is a mixture of $\mathrm{CF}_{4}(90 \%)$ and $\mathrm{CH}_{4}(10 \%)$ which provides a maximum drift time of $60 \mathrm{~ns}$ (for the electrons created in the process of ionisation) [50]. Fig. 2.25 shows the layout of MDT's in A-layer and Fig. 2.26 shows an unit of MDT.

Each layer of MDT's in the Forward Muon system is accompanied by a layer of scintillator pixels. The counters with a $\phi$ segmentation of $4.5^{0}$, are used for triggering as well as for rejecting the cosmic muons. 


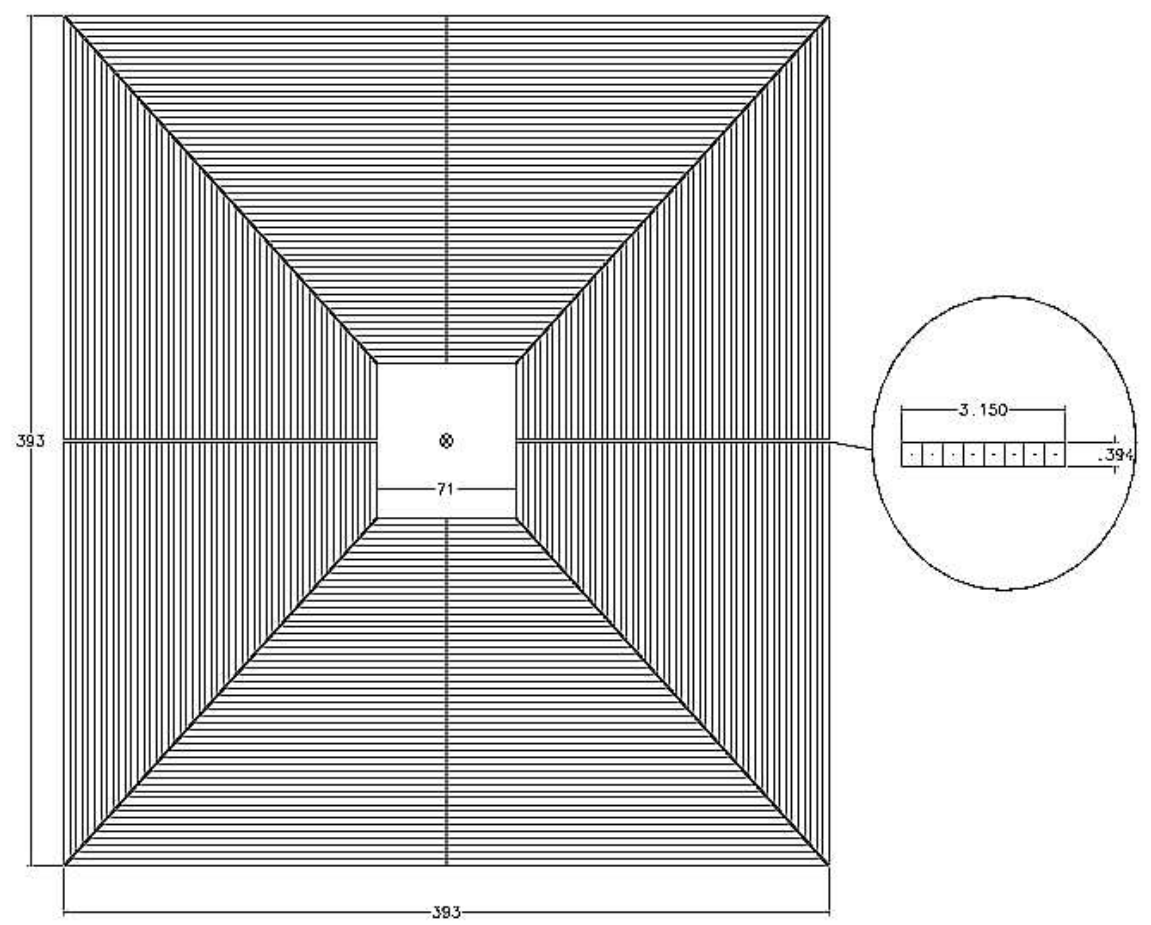

Figure 2.25: Design of A-layer MDT's.

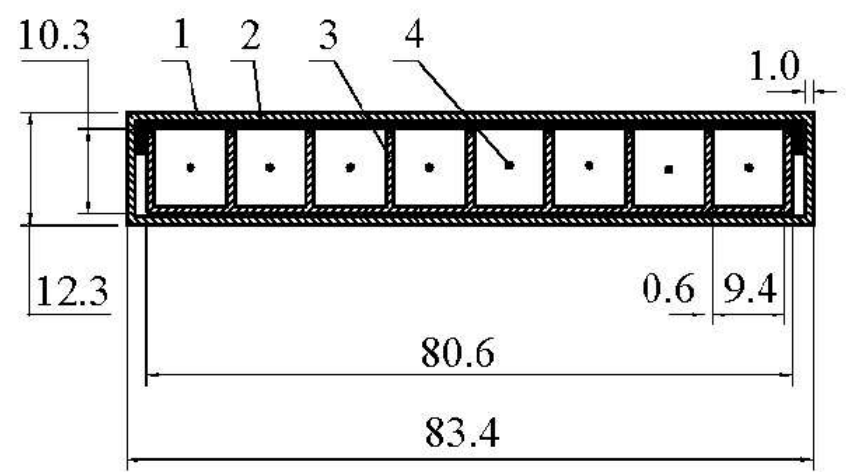

Figure 2.26: A 8-cell unit of MDT (dimensions are in the units of millimetre.). 


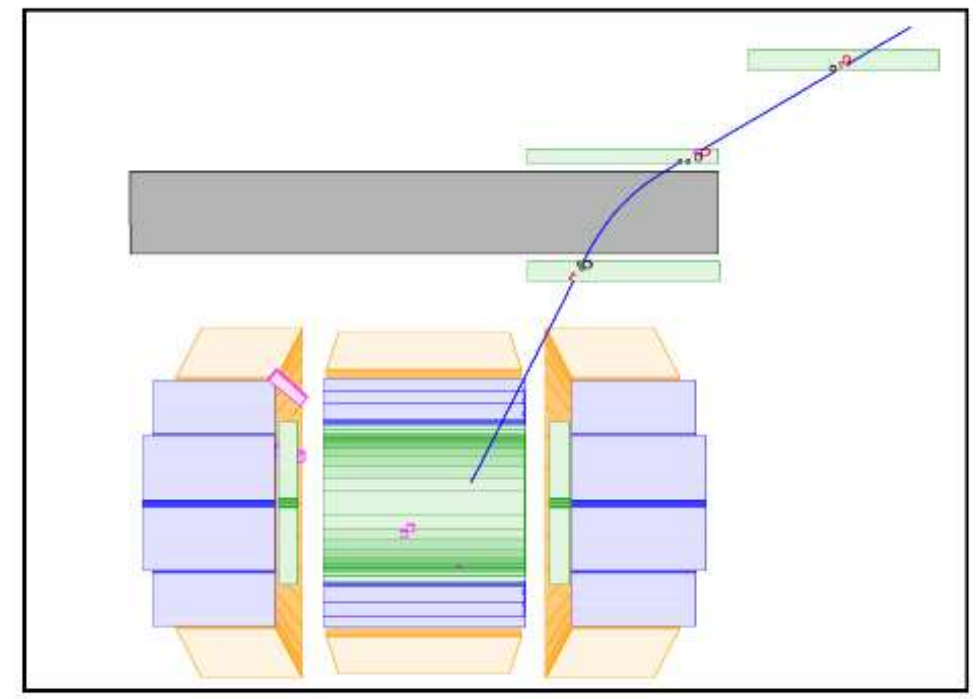

Figure 2.27: A simulated muon trajectory through DØ detector.

\section{Performance of the Muon System}

As mentioned earlier, due to bending of muon trajectories in $r-z$ plane under the influence of a toroidal magnetic field (Fig. 2.27), it is possible to measure the muon momenta using only the muon system information. The momentum resolution of the tracks measured in the muon system is shown in Fig. 2.28 [51]. The parametrization as a function of the muon momentum is given by

$$
\frac{\sigma(1 / p)}{(1 / p)}=\frac{\alpha \cdot(p-\beta)}{p} \oplus \gamma \cdot p
$$




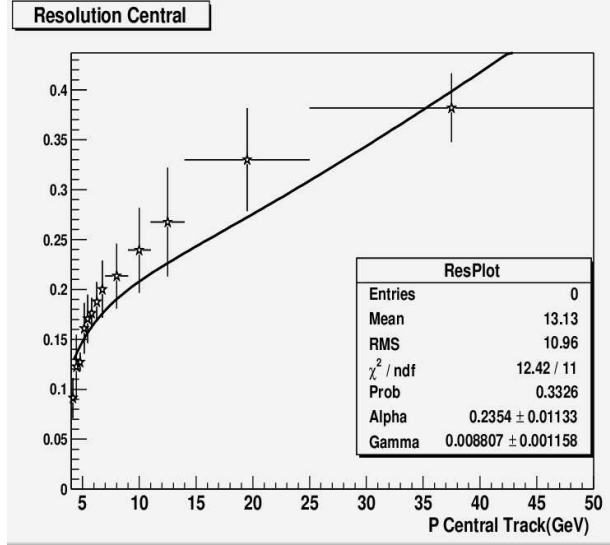

(a)

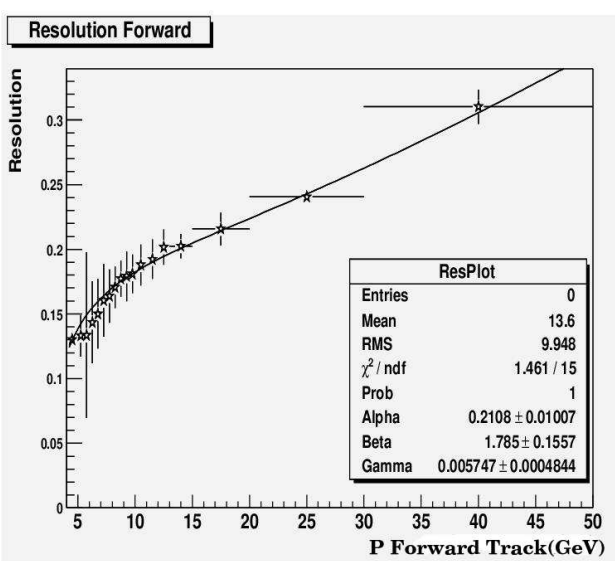

(b)

Figure 2.28: Local muon momentum resolution as a function of muon momentum, $p$ for central (a) and forward (b) regions.

where,

$\alpha=0.36 \pm 0.04, \quad \beta=3.1 \pm 0.2 \mathrm{GeV}, \quad \gamma=0.0050 \pm 0.0026 \mathrm{GeV}^{-1}$ (Central)

$\alpha=0.21 \pm 0.01, \quad \beta=1.79 \pm 0.16 \mathrm{GeV}, \quad \gamma=0.0057 \pm 0.0005 \mathrm{GeV}^{-1}$ (Forward)

\subsubsection{Trigger and Data Acquisition System}

The Tevatron provides D $\varnothing$ with 36 bunch crossings every $21 \mu$ sec or $1.7 \times 10^{6 \dagger}$ crossings per second. A typical event contains $\approx 250$ kilobytes of information from all readout channels of the $\mathrm{D} \varnothing$ detector, which corresponds to $\approx$

\footnotetext{
${ }^{\dagger}$ As mentioned earlier, in Tevatron the bunches of protons or antiprotons are not loaded uniformly. In the present scenario of Run IIa, the collision rate is $2.5 \mathrm{MHz}$ for the minimal bunch-crossing time of 396 ns.
} 
500 Gigabytes of data per second, if recorded by DØ. Moreover, most of the $p \bar{p}$ collisions result in inelastic scattering processes and only a small fraction of these interactions produce particles that are of interest to the $\mathrm{D} \varnothing$ experimenters.

In order to select and record the results only from the interesting collisions, a complex Trigger and Data Acquisition System is utilised by DØ. The system has three levels of increasingly sophisticated event characterisation, called Level 1, Level 2 and Level 3 respectively in the sequence of filtering. Implementation of a list of triggers in the trigger and data acquisition, helps in recording the data to tapes at a comfortable rate of $\approx 50$ events $/$ sec. $\mathrm{A}$ typical trigger is primarily motivated to select the events from a particular type of physics process viz., QCD, high $p_{T}$ di-lepton events, etc. It is composed of a set of selection criteria applied at different levels of triggering. For example, the present analysis uses the $\mathbf{e} \mu$ trigger (see Sec. 4.2), which requires an electron and a muon at the first level of triggering (Level 1), while more stringent criteria on shower profile (in calorimeter) of the selected electron, is applied at the final level of triggering (Level 3). Level 1, Level 2 and Level 3 trigger systems are described briefly in the remaining sections of this chapter. A simplified schematic diagram of D $\varnothing$ Trigger and Data Acquisition System is presented in Fig 2.29. 


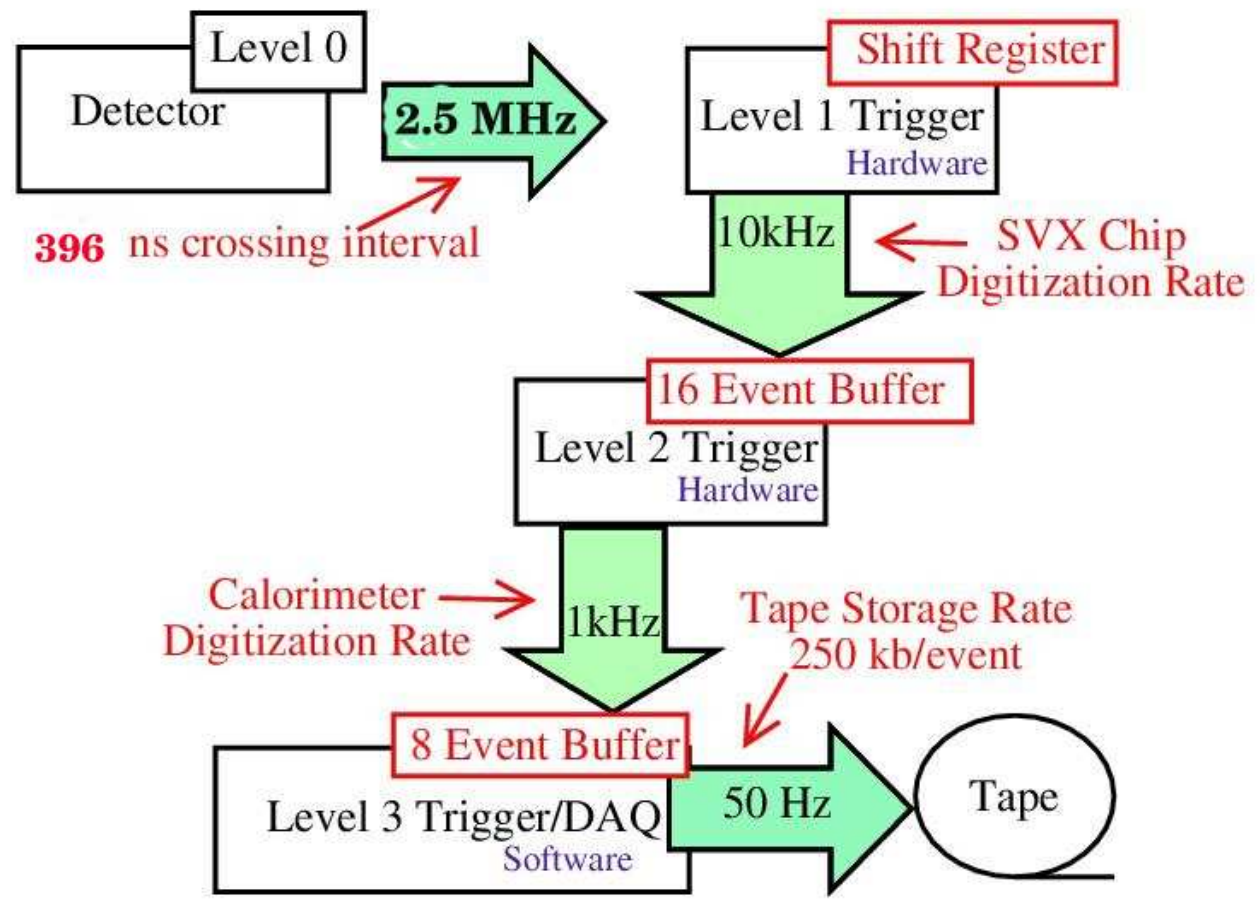

Figure 2.29: Schematic illustration of $\mathrm{D} \varnothing$ tri-level trigger system. 


\section{Level 1}

The Level 1 trigger condition is made by a hardware trigger system using pipe-lined architecture with an overall dead time of $4.2 \mu$ sec. L1 trigger includes information from the CFT and CPS (called Central Track Trigger or CTT), FPS, Calorimeter and Muon detectors. Digitizing readout systems for different sub-detectors are provided with sufficient memory to retain the data corresponding to 32 bunch crossings. Each Level 1 processor examines the raw signals from different sub-detectors and does some logical operations to produce trigger bits $^{\S}$ corresponding to objects with physical potential. For example, one might require a Calorimeter trigger tower (of size $0.2 \times 0.2$ in $\eta-\phi)$ with $E_{T}$ above a certain threshold, hit patterns that form tracks with $p_{T}$ above a certain threshold in CFT, a muon in central or forward muon system, and so forth. In order to have fast triggering and minimum processing time, Level 1 trigger system does not apply any quality cuts on the triggered objects. The Level 1 framework, built of Field Programmable Gate Arrays (FPGA's), passes trigger bits to the next level i.e., Level 2 for the "accepted" events at a rate of nearly $2 \mathrm{kHz}$ [52].

\footnotetext{
§There are 128 trigger bits pre-defined in the Level 1 framework. Level 1 framework accepts an event if any one of these bits are set.
} 


\section{Level 2}

The Level 2 trigger is again a hardware based trigger system and correlates the Level 1 trigger decisions with the detector signals, some of which are not used in Level 1 [53, 54]. It uses 5 Compaq Alpha processors to analyse signals from different sub-detectors, Calorimeter, Muon, Preshower, Central Track Trigger (CTT) and Silicon Track Trigger (STT) processors. Calorimeter processor is assigned to search for jets or electron/photon in calorimeter data. The Muon processor performs the search for muons in central and forward muon detectors. Preshower and CTT processors combine information from CPS and FPS, and CFT and CPS respectively, while STT processor runs tracking algorithms using SMT and CTT data. In addition, event-wide variables, such as the total transverse energy, and event correlations such as $\phi$ separation between the objects are computed at Level 2. The final trigger decision is made by another processor called, Level 2 Global processor. A schematic diagram showing the data flow in Level 1 and Level 2 is depicted in Fig. 2.30. As in Level 1, the Global processor makes the trigger decisions in 128 predefined bits, while the system is capable of holding 16 Level 1 accepted events at the most. Level 2 has an output rate of $1 \mathrm{kHz}$ with a maximum latency of $100 \mu$ sec. 


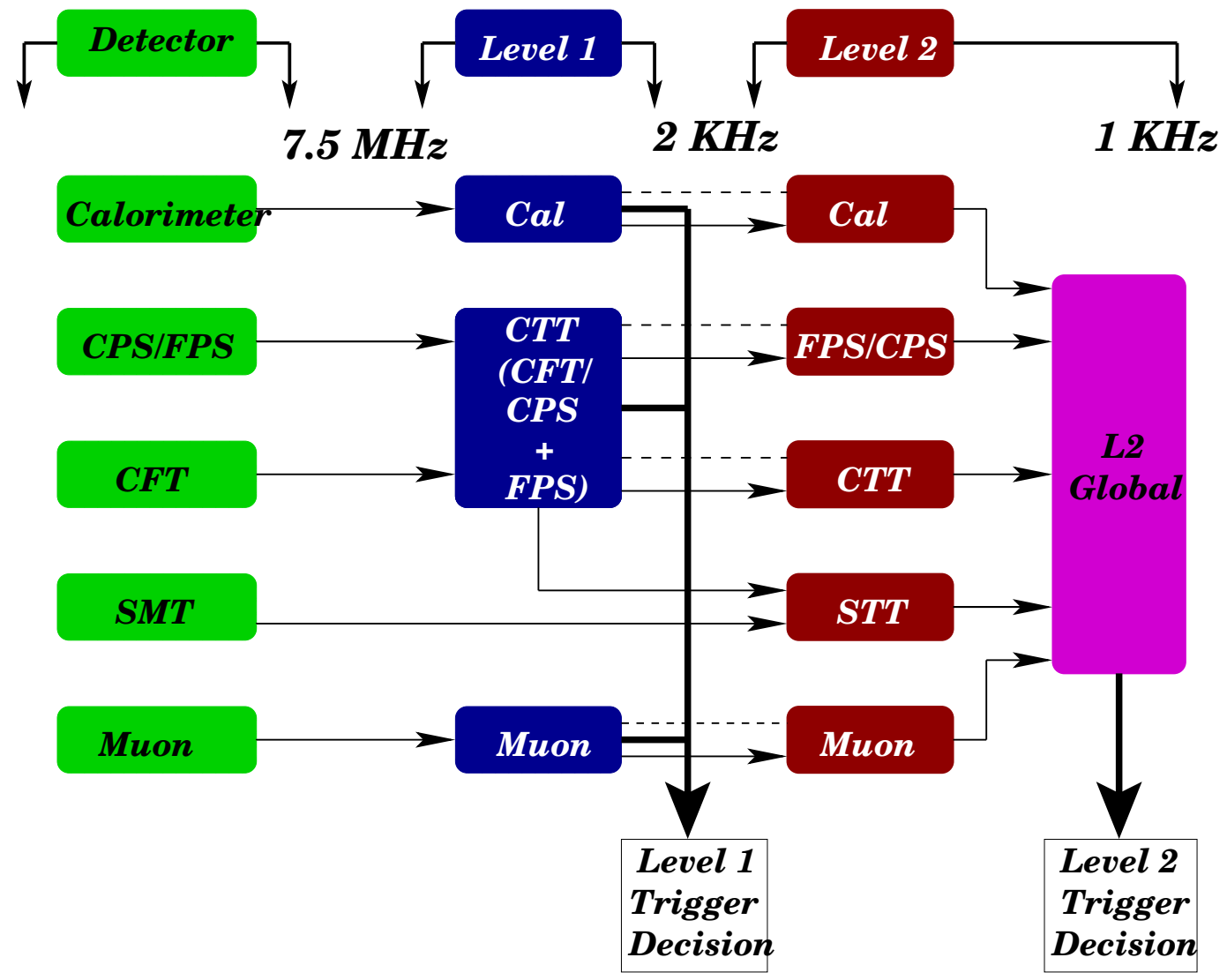

Figure 2.30: Block diagram illustrating the components of Level 1 and Level 2 , and the connections between them. 


\section{Level 3}

Level 3 Trigger is a software based trigger, running on a farm of $82 \mathrm{PC}$ 's, using Linux Operating system (Level 3 Farm). Once an event is accepted by Level 2 , the digitized data corresponding to that particular event from all channels of the entire $\mathrm{D} \emptyset$ detector system, is passed to the Level 3 Trigger system. Information from each detector is collected in single-board-computers (SBC), which send this information to individual nodes of Level 3 farm, through commercial Ethernet switches. Due to the time constraint ( $\leq 100 \mathrm{~ms} /$ event), each event is partially reconstructed ${ }^{\dagger}$ in a single node, to determine whether the event satisfies the criteria for acceptance. Level 3 has a large rejection factor of 20 that is required to limit the output rate to less than $50 \mathrm{~Hz}$. This is accomplished through the implementation of Level 3 trigger conditions that impose quality criteria on the objects e.g., shower shape criteria for an electron or a jet candidate. Moreover, Level 3 is capable of applying more stringent cuts (compared to Level 2) on the event-wide variables such as missing transverse energy $\left(\mathbb{E}_{T}\right)$, total transverse energy, etc. in summary, the flexibility of the triggering conditions is expanded in Level 3, by allowing the trigger decisions to be stored in 256 bits [55], where each bit corresponds

\footnotetext{
${ }^{\dagger}$ Full reconstruction of $\mathrm{D} \varnothing$ data is performed offline. This is explained in great detail in Chap. 3.
} 
to one or more Level 1/Level 2 trigger bits. 


\section{Chapter 3}

\section{Event Reconstruction}

The particles produced in $p \bar{p}$ collisions generate signals in various parts of the $\mathrm{D} \varnothing$ detector. The raw data in the form of analog and digital signals from various sub-systems are stored in tapes. The process of converting this information into a suitable form for physics analysis such as kinematic parameters of the physics objects such as leptons, jets, etc., is known as "Event Reconstruction". Information from a collision (event) is stored, only if the kinematic properties of the physics objects satisfy a set of criteria, called trigger. Detailed and more accurate reconstruction of the physics objects is performed later by a number of parallel processing computers, collectively called a farm. At D $\varnothing$ the event reconstruction is carried out by a software package called $d \emptyset r e c o$. In this chapter, main features of $d \emptyset r e c o$ as well 
as the techniques used for identifying electrons, muons, jets, and neutrinos (missing $E_{T}$ ) are described.

\subsection{The DØ Reconstruction Program}

The reconstruction programme dØreco is a collection of numerous algorithms, which are used to build up an accurate estimate of the kinematics of a $p \bar{p}$ interaction. The code for this package has been written in $\mathrm{C}++$ Programming Language and is executed in an Object-Oriented (OO) software environment. Since the beginning of Run II, the package has been modified several times. Each version is named and stored separately as a new production release. The raw data are processed routinely with every version of production release. This analysis uses the data processed with "p14" version of $d \emptyset r e c o$.

dØreco performs three major tasks. First, the signal from all channels of the central tracking system are converted into spatial location of hits, and the signals from all cells in the calorimeter are converted into energy deposits. Secondly, the hits from the tracking system are joined to form tracks, while the calorimeter cells are grouped into clusters of energy. The signals from different layers of muon system are combined to find the signa- 
ture of a muon. Finally, information from the tracking system, calorimeter and muon chambers are combined to reconstruct jets, and to identify electron and muon candidates. The criteria used by dØreco to identify electrons, muons or jets are quite loose and further quality cuts are applied during the analysis, specific to a physics topic. Output of dØreco is stored in two types of format:

- Data Summary Tape (DST) - contains the raw data plus the output from the full reconstruction. In DST format, size of an event is typically 150 kilobytes and data are stored on large magnetic tapes. DST's are used primarily for graphical event display and for re-reconstruction of events with later versions of $\mathrm{d} \emptyset$ reco, as they contain raw data from the detector.

- Thumbnail (TMB) - contains a compact version of the information from full reconstruction. The size of an event in this case is 10 kilobytes. Thumbnail contains relevant information for each individual physics object which can be used very easily and thus used for studying specific physics topics. 


\subsubsection{Track Reconstruction}

While moving in a helical trajectory under the influence of an uniform magnetic field, a charged particle produces hits in various layers of the central tracking system. Track reconstruction code correlates these hits to identify a track, left by the charged particle while passing through the tracking system. This process is accomplished in two steps: hit reconstruction, in which the signals from individual channels of a particular layer are grouped (in case of ambiguity); and track finding where the clusters from different layers are combined using certain algorithms to build the physical tracks of the charged particles.

\section{Hit Reconstruction}

In Central Fibre Tracker (CFT), the passage of a charged particle through a single layer, illuminates either one or two fibres. Such adjacent fibres are grouped together to form a cluster. If more than two illuminated adjacent fibres in a single layer are found, every possible pair is considered as an individual cluster; the ambiguity is resolved by the tracking algorithm. A detailed description can be found in Ref. [56].

Similarly while traversing the Silicon Micro-strip Tracker (SMT), a 
charged particle produces signal in one or two strips, depending on the angle of incidence. A simple grouping of the adjacent strips, with signal above the noise level, is called a cluster. The position of the cluster is the average of the adjacent strip positions, weighted by the deposited charge in each strip. These clusters or hits are used by the tracking algorithm to reconstruct particle trajectories.

\section{Track Finding}

Track finding refers to the procedure of building tracks from a group of hits and can be divided into two steps: "Pattern recognition" and "track fitting". In Pattern recognition, a list of hits which could lie along a physical path are selected. In $\mathrm{D} \varnothing$, a typical event consists of very large $\left(10^{4}-10^{6}\right)$ number of hits and a simple combinatorial approach would consume large amount of CPU time. Therefore, optimized algorithms are very important for pattern recognition. Moreover, the algorithm has to be optimized for different situations. For example,

- charged lepton identification for isolated high momentum $\left(p_{T}>20\right.$ $\mathrm{GeV}$ ) tracks as well as in hadronic environment with relatively low momentum $\left(p_{T}>1 \mathrm{GeV}\right)$ tracks from heavy flavour decay. 
- separation of prompt electron or positron tracks from those, originating from photon conversion in the tracking system.

Once a track is reconstructed from the hits in different layers of the tracker, a $\chi^{2}$ (between the fitted track and the individual hits contributing to the track) is estimated in order to determine the quality of the track.

DØ currently uses two algorithms for track finding. The first one is known as Histogramming Track Finder (HTF). In this method, for each pair of hits in coordinate space $(\mathrm{x}, \mathrm{y})$, all possible trajectories can be transformed into a straight line in parameter space $\left(\rho^{\dagger}, \phi\right)$. The parameter space of $-\rho_{0}<\rho<\rho_{0}\left(\rho_{0}=q B / p_{t}^{\min }\right.$, where $\mathrm{p}_{\mathrm{t}}^{\min }$ is the minimum track $p_{t}$ under consideration), $0<\phi<2 \pi$ is divided into the cells of a 2 dimensional histogram and for each pair of hits, the contents of the cells are increased correspondingly. All hits from the same track will end up in the same cell, while the hits from different tracks will fall in different cells randomly. Fig. 3.1 illustrates the algorithm with a single track of $p_{T}=1.5 \mathrm{GeV}$, consisting of 5 hits in the central tracking system. This method is called Hough Transform Technique [57]. A search algorithm looks for peaks in the parameter space and each peak corresponds to a candidate track. These track candidates are

\footnotetext{
${ }^{\dagger} \rho=q B / p_{t}$, where $\mathrm{q}=$ charge, $\mathrm{B}=$ magnetic field, and $p_{t}=\sqrt{p_{x}^{2}+p_{y}^{2}}$ is the transverse momentum.
} 
then fitted with Kalman Filter [58].

The second algorithm, known as Alternate Algorithm Track (AATrack), builds track hypothesis with 3 initial SMT or CFT (if there are no SMT hits) hits. The first innermost hit is used to determine a $\phi$-window where the second hit is searched for (see Fig. 3.2). The third hit is found by matching a hit in next layer provided that, the hypothesis for a track with these three hits, has a radius less than $30 \mathrm{~cm}$ (corresponding to a minimum $p_{T}$ of 180 $\mathrm{MeV}$ ) and axial impact parameter less than $2.5 \mathrm{~cm}$ w.r.t. beam spot as shown in Fig. 3.2. The hypothesis is also required to have a $\chi^{2}$ less than a cut-off maximum value. The 3-hit track hypothesis is then expanded to other layers of the tracker where the hits are matched, that fall in a window around the initial hypothesis as illustrated in Fig. 3.3. The track candidates are then filtered based on various parameters of the track e.g., number of SMT hits, total number of hits, etc. The surviving candidates are fitted to get the coordinates and momenta of the particle trajectories.

The common tracks as well as the tracks reconstructed by either of these two algorithms are considered as the final set of tracks. Each reconstructed track is associated with 5 parameters:

- DCA (Distance of Closest Approach): minimal distance between the 
(a)
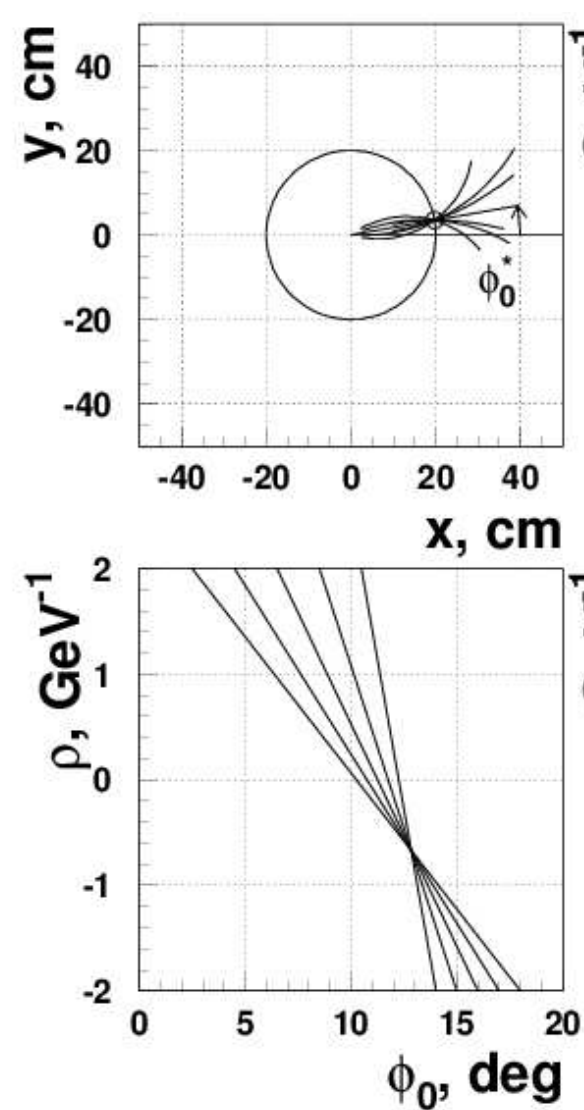

(c) (b)
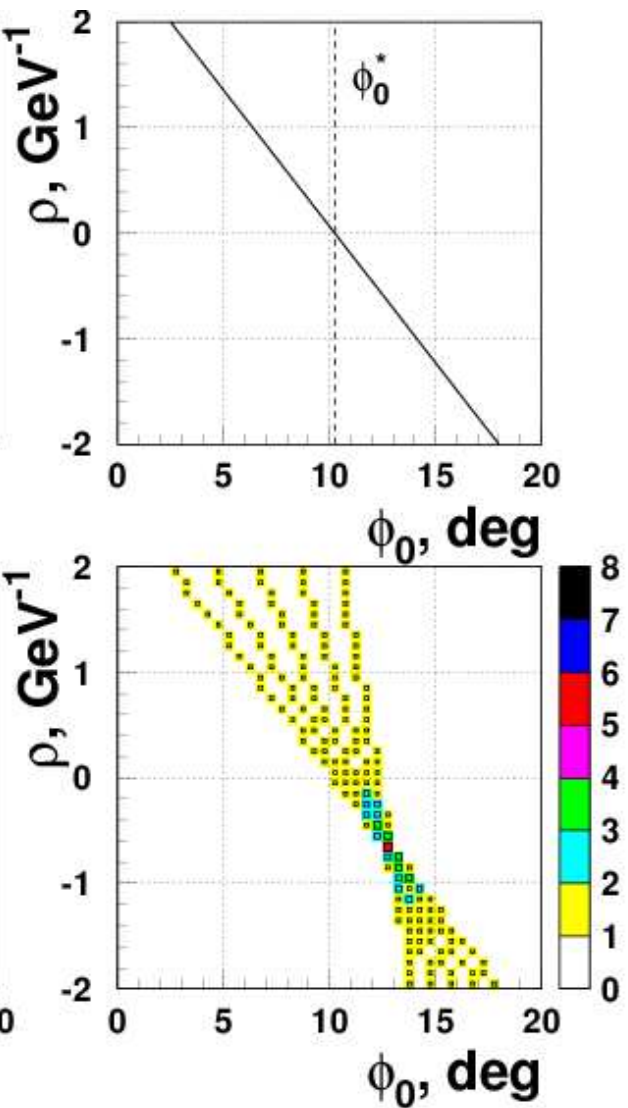

(d)

Figure 3.1: Illustration of Histogramming Track Finder with a single track with $p_{T}=1.5 \mathrm{GeV}$, consisting of 5 hits.(a) a set of trajectories in coordinate space with a given hit, originating from the geometric centre of the detector; (b) a straight line in parameter space describing all possible trajectories from a single hit. (c) 5 such straight lines in parameter space, from the 5 hits; the point of intersection of these straight lines corresponds to the track parameters. (d) The point of intersection corresponds to a peak in a 2 dimensional histogram in $\left(\phi, \rho_{0}\right)$. 


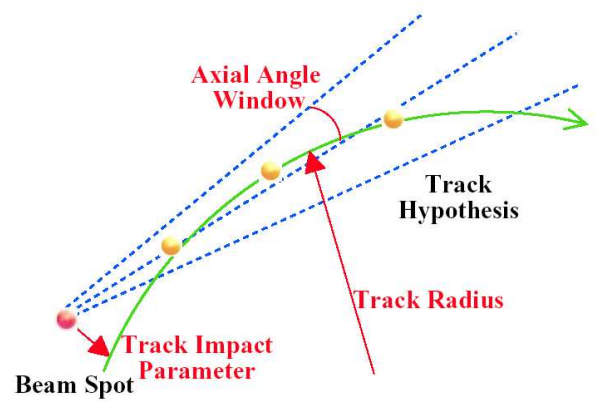

Figure 3.2: Illustration of the different variables to reconstruct initial track hypothesis out of three hits in AAtrack algorithm.

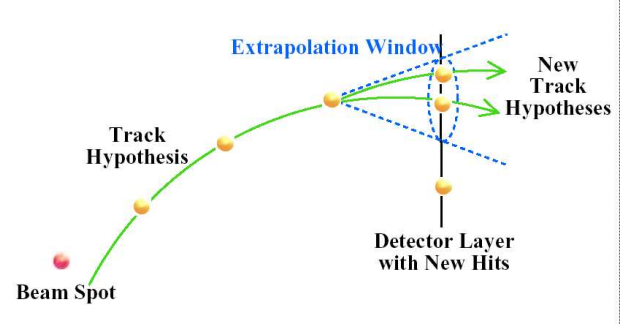

Figure 3.3: Expansion of track hypothesis, built out of three initial hits to the other layers of tracker, in AAtrack algorithm. 
track helix and the z-axis in $\mathrm{x}-\mathrm{y}$ plane. The position at which the DCA is calculated, is called the point of closest approach.

- $z_{0}$ : z-position of the track at the point of closest approach.

- $\phi$ : direction of the track in the transverse plane at the point of closest approach.

- $\tan \lambda$ : tangent of $(\pi / 2-\theta), \theta$ being polar angle of the trajectory at $z_{0}$.

- $\frac{q}{p_{T}}: q$ and $p_{T}$ are the charge and transverse momentum of the trajectory.

For detailed description of the DØ tracking algorithms, Refs. [59] and [60] can be consulted.

\subsubsection{Event Vertex Determination}

During $p \bar{p}$ bunch crossings, the point of collision, called "interaction vertex" changes position on an event-by-event basis, in both transverse and longitudinal directions. The $z$ position of the interaction vertex varies widely, with a roughly Gaussian distribution of width $\sim 25 \mathrm{~cm}$ while in the transverse plane, position of the interaction vertex is limited by the size of the beam $\operatorname{spot}(\sim 40 \mu)$. 
In order to estimate the transverse and the longitudinal components of the momentum for each physics object properly, one needs to locate the position of the interaction vertex accurately. Moreover, during Run II of Tevatron, in a particular bunch crossing, more than one interaction can occur. Thus a hard-scattered event which produces the $t \bar{t}$ pair, can be accompanied by one or more low- $p_{T} p \bar{p}$ interaction (called minimum bias interaction, such as low $p_{T}$ QCD scattering, single diffraction and double diffraction). In order to get back the proper kinematics of the hard-scattered event, one needs to be able to separate different interaction vertices in an event.

DØ currently uses two vertex finder algorithms, called "dØreco" and "dØroot". Both algorithms first select the tracks with very loose criteria (dca significance $<100$ with respect to the geometric centre of the detector i.e., $(0,0)$ in $(r, \phi))$, to find the approximate location of the beam spot in $(r, \phi)$ with respect to $(0,0)$. Next, dØreco fits all the tracks with dca significance $<5$ with respect to $(0,0)$, to find a common vertex position by minimising the impact parameter (based on least square fit method) of the tracks. After fitting all tracks into a vertex, the track with highest $\chi^{2}$ contribution to the vertex is removed and the vertex is refitted. The process is iterated until the total vertex $\chi^{2}$ is smaller than 10 . Once a vertex is found, the algorithm starts over with the remaining tracks to find more vertices and thus a first 
list of vertices are formed. More tracks having dca significance $<5$, now with respect to the beam spot (located previously), are attached to each of these vertices. For each of the first pass vertices, the tracks are refitted again to form the final set of vertices, following the same procedure applied in search of the first pass vertices.

After locating the beam spot (identical to dØreco), "dØroot" utilizes a $z$-clustering algorithm to distinguish between the tracks belonging to different interaction vertices. The tracks, within $2 \mathrm{~cm}$ from one another in the z-direction, are clustered first and all selected tracks are required to have dca significance $<3$ with respect to the nominal position of beam spot. With the tracks belonging to the highest multiplicity cluster, a global fitting is performed using a Kalman Filter technique to find a vertex. Here the track impact parameter and the difference in momenta (original track momentum and the propagated track momentum with respect to the reconstructed vertex) are minimized simultaneously. The track with the highest $\chi^{2}$ contribution to the vertex is then removed from the vertex and the process is iterated in a fashion identical to $\mathrm{d} \emptyset$ reco. Despite the differences in trackselection procedure and clustering of tracks, both the algorithms are found to have comparable performance for both data and Monte Carlo events [61].

The remaining task of the vertex reconstruction is to identify the hard- 
scattered vertex (called Primary Vertex or PV) from the list of vertices produced by "dØreco" or "dØroot". Several discriminating variables, for ex-

ample, $\sum \log p_{T}^{\text {track }}$, have been tried to isolate the PV's from minimum bias vertices. Based on prior knowledge from Monte Carlo events, the probability that a vertex would be a hard scattered vertex can be estimated and the highest probability vertex is considered as the PV in an event. Further details are given in Ref. [63, 63].

\subsubsection{Calorimeter Reconstruction}

The energy of a electron/photon or a hadronic jet is measured precisely through reconstruction of calorimeter data. Reconstruction begins with conversion of the ADC counts recorded in each calorimeter cell (having a signal $2.5 \sigma$ above the noise level), into a value for the energy deposited. The conversion factor from digitized counts to $\mathrm{GeV}$ comes from the results of test beam experiments, in which portions of the calorimeter were exposed to electron and pion beams of known energies. These measurements have been done prior to installation of the calorimeter in the $\mathrm{D} \varnothing$ detector. The response of the calorimeter modules to these mono-energetic electron/pion beams are stored in the database. 
However, the test beam experimental setup can not reproduce the exact condition that exists at the time of data-taking (the amount of upstream material and the length of cables are different in the actual experiment from that in the test beam setup), and thus it is necessary to perform an in situ calibration. Since the beginning of Run II, various calibration methods have been proposed to improve the precision of calorimeter calibration. For example, $\phi$-symmetry of the total activity in an event, which should be same for all cells in one floor at a particular pseudo-rapidity $(\eta)$, can be utilized. Currently, calorimeter signals are corrected for time-dependent changes in the gains and pedestals of the readout channels for each cell. Calibration runs are routinely taken during periods when there are no collisions and the information is stored in the database. For each cell in the calorimeter, corresponding database entry is accessed by the reconstruction program to convert cell counts to energy.

Once the energy deposited in each cell is determined, signals from all the cells in different calorimeter layers, with the same $\eta$ and $\phi$ indices (collectively called a tower) are summed. While taking this sum, it is assumed that each cell represents a massless particle. As energy and momentum are equivalent under this assumption, each cell is assigned an energy-momentum four-vector $(E, E \sin \theta \cos \phi, E \sin \theta \sin \phi, E \cos \theta)$, where $E$ is the energy deposit in the 
cell and $\theta(\phi)$ is the polar (azimuthal) angle defined by the cell centroid w.r.t. the reconstructed primary vertex. The tower energy-momentum four-vector is then given by the sum of the four-vectors of all cells in the tower. Once this four-vector is assigned, the $(\theta, \phi)$ coordinates of the tower are calculated as,

$$
\begin{aligned}
\phi_{\text {tower }} & =\tan ^{-1} \frac{E_{\text {tower, } \mathrm{y}}}{E_{\text {tower }, \mathrm{x}}} \\
\theta_{\text {tower }} & =\tan ^{-1} \frac{\sqrt{E_{\text {tower }, \mathrm{x}}^{2}+E_{\text {tower, } \mathrm{y}}^{2}}}{E_{\text {tower } \mathrm{z}}} \\
\eta_{\text {tower }} & =-\ln \left(\tan \frac{\theta_{\text {tower }}}{2}\right)
\end{aligned}
$$

where $E_{\text {tower,x }}, E_{\text {tower,y }}$ and $E_{\text {tower,z }}$ are the x, y and z components of tower energy, respectively.

\subsection{Particle Identification}

\subsubsection{Electrons}

Electrons and photons are identified by detection of clusters of cells, having large energy deposition in electro-magnetic layers of the calorimeter, with (electron) or without (photon) an associated track in the central tracking 
system.

\section{Candidate Reconstruction}

The first step in the reconstruction of electrons and photons is to group the towers with significant energy deposition in the electromagnetic part of the calorimeter, into clusters of energy. Starting with the highest- $E_{T}$ tower, all neighbouring towers above an $E_{T}$ threshold of $1 \mathrm{GeV}$ are added to the cluster, and the process repeats until no towers neighbouring the cluster are above the energy threshold. A new cluster is then started from the highest- $E_{T}$ tower not previously assigned to a cluster.

Any such cluster in the calorimeter with more than $90 \%$ of its energy in the electromagnetic layers of the calorimeter (and more than $40 \%$ in a single tower) is identified by the reconstruction program as an electron or a photon candidate. This cut alone removes most of the hadronic clusters while retaining more than $99 \%$ of true electrons and photons because a typical hadronic jet has a broader energy distribution in the lateral direction with only about $10 \%$ energy deposition in the electromagnetic layers.

As described in Sec. 2.2.6, the electron/photon gives rise to electromagnetic shower in the calorimeter and the centroid of the shower is com- 
puted using the cells in the third electro-magnetic layer ${ }^{\ddagger}$ of the calorimeter.

The centroid is defined as:

$$
\vec{x}_{c o g}=\frac{\sum_{i} \vec{x}_{i} w_{i}}{\sum_{i} w_{i}}
$$

where the sums extend over all cells in the third layer of EM calorimeter, which contribute to the shower cluster, $\vec{x}_{i}$ is a vector from the primary vertex to the ith cell centroid, and

$$
w_{i}=\max \left(0, w_{o}+\ln \left(\frac{E_{i}}{\sum_{i} E_{i}}\right)\right) .
$$

The logarithmic weighting reflects the logarithmic development of a shower in lateral direction and the $w_{o}$ is chosen empirically by optimizing the position resolution. The transverse resolution of the center of gravity is measured to be about $2.5 \mathrm{~mm}[64]$.

Finally, the reconstruction program searches for a central track pointing from the primary vertex to the cluster within a road size of $\Delta \eta \times \Delta \phi=$ $0.1 \times 0.1$. If such a track is found, the cluster is identified as an electron candidate; otherwise it becomes a photon candidate.

\footnotetext{
${ }^{\ddagger}$ The third layer of electromagnetic calorimeter has the finest granularity.
} 


\section{Selection Criteria}

Sources of background processes that can mimic an electron are:

- $\pi^{0}$ decay to two photons, producing an electromagnetic cluster, which overlap randomly with a track from a nearby charged hadron.

- photon conversion to $e^{+} e^{-}$pairs early in the tracking system.

- Sufficient energy deposited in the electromagnetic part of the calorimeter due to fluctuations in the energy deposition of a haronic jet.

In order to suppress these backgrounds while retaining high efficiency for identifying true electrons, information from the calorimeter and the tracking system are combined. Following quantities improve electron identification:

\section{Cluster EM fraction:}

$$
f_{E M}=\frac{\text { Energy in the EM section }}{\text { Total Energy }}
$$

This value is required to be greater than or equal to 0.90 for electron and photon candidates. 


\section{Cluster isolation:}

$$
f_{\text {iso }}=\frac{E(0.4)-E M(0.2)}{E M(0.2)}
$$

where $\operatorname{EM}(0.2)=$ the electromagnetic energy within a cone of radius $\Delta R=\sqrt{\Delta \eta^{2}+\Delta \phi^{2}}=0.2$ centered on the cluster and $\mathrm{E}(0.4)=$ the total energy contained within a concentric cone of radius $\Delta R=0.4$. Any cluster with $f_{\text {iso }}>0.15$ is rejected.

\section{Cluster Shape:}

The development of electromagnetic and hadronic showers as electrons (or photons) and jets travel through the calorimeter, are sufficiently different. Therefore, shower shape information can be used to differentiate between electrons (or photons) and hadrons. D $\varnothing$ uses both longitudinal and transverse profiles of the shower for such distinction. A covariance matrix technique is used to compare the shape of a given shower with the shower shape expected for electrons determined from both the test-beam data and Monte Carlo. The covariance matrix, for a sample of $\mathrm{N}$ electrons or photons is defined as,

$$
M_{i j}=\frac{1}{N} \sum_{n=1}^{N}\left(x_{i}^{n}-\left\langle x_{i}\right\rangle\right)\left(x_{j}^{n}-\left\langle x_{j}\right\rangle\right),
$$


where $x_{i}^{n}\left(x_{j}^{n}\right)$ is the value of observable $i(j)$ for nth electron (or photon) and $\left\langle x_{i}\right\rangle\left(\left\langle x_{j}\right\rangle\right)$ is the mean value of the observable $i(j)$ for the sample. A total of 7 variables are used.

- The fractions of the total energy contained in the EM layers 1, 2, 3 and 4 , of the calorimeter

- Total energy deposited in the EM layers

- z-position of the primary vertex

- Shower width in transverse direction

Once $M$ has been calculated, the degree of agreement between a specific shower and that expected from an electron is defined by:

$$
\operatorname{Hmx} 7=\sum_{i, j=1}^{7}\left(x_{i}-\left\langle x_{i}\right\rangle\right) H_{i j}\left(x_{j}-\left\langle x_{j}\right\rangle\right)
$$

where $H$ is the inverse matrix of $M$. A cut of $\operatorname{Hmx} 7<50$ is applied for electromagnetic shower selection.

\section{Track Matching Significance:}

To reject random track overlaps with the electromagnetic showers, the consistency between the direction of the candidate track and the shower centroid (determined using Eqn. 3.4) is calculated. The track matching 
significance for clusters is estimated by calculating the $\chi^{2}$ probability for 2 degrees of freedom, $\mathrm{P}\left(\chi_{\mathrm{EM}-\mathrm{TRK}}^{2}, 2\right)$ where,

$$
\chi_{\mathrm{EM}-\mathrm{TRK}}^{2}=\left(\frac{\Delta \phi}{\sigma_{\Delta \phi}}\right)^{2}+\left(\frac{\Delta z}{\sigma_{\Delta z}}\right)^{2},
$$

where $\Delta \phi(\Delta z)$ is the mismatch in variable $\phi(z)$ between the shower centroid and the track direction, and $\sigma_{\Delta \phi}\left(\sigma_{\Delta z}\right)$ is the error on $\Delta \phi(\Delta z)$. Present analysis does not require any explicit cut on this Probability, but this information is used as one of the inputs to calculation of the "Electron Likelihood" described later.

5. $\mathbf{E}_{\mathbf{T}}^{\mathrm{Cal}} / \mathbf{p}_{\mathbf{T}}^{\text {trk }}:$

To discriminate between electrons coming from the interaction vertex and those from photon conversions, this is an useful variable. The observable here is the ratio of the transverse energy of a candidate cluster in calorimeter to the transverse momentum of the track, matched with the cluster. In case of photon conversion there could be a second random track very close to the EM cluster which could be resolved by the tracker. Generally such a track would be of low $p_{T}$ and in this case $E_{T}^{\mathrm{Cal}} / p_{T}^{\text {trk }}$ would tend to be large. This analysis does not apply any cut on this observable, but it is used as one of the inputs to "Electron 
Likelihood".

\section{Electron Likelihood:}

In order to distinguish real electrons from fake ones, certain characteristics of the fakes must be considered. For example, a $\pi^{0}$ is typically produced in association with charged hadrons. Because of this, the calorimeter can be used to pick up signs of hadronic activity around the electro-magnetic cluster. Moreover, since $\pi^{0}$ would have to overlap a track from one of the charged hadrons to get selected as an electron, track-matching probability of the cluster could be poor. The track would not necessarily be isolated and $E_{T}^{\mathrm{Cal}} / p_{T}^{\text {trk }}$ would not tend towards 1, as expected for good electrons. Considering all the above mentioned characteristics a 7-parameter likelihood is constructed. Inputs to this likelihood are

- $f_{\mathrm{EM}}($ see Eqn. 3.6)

- $\operatorname{Hmx} 7$

- $E_{T}^{\mathrm{Cal}} / p_{T}^{\mathrm{trk}}$

- $\operatorname{Prob}\left(\chi_{\text {Spatial EM-trk }}^{2}, 2\right)$ (see Eqn. 3.10)

- Distance of closest approach to PV of the candidate track 
- Number of tracks in a $\Delta R<0.05$ cone around the candidate track

- $\sum p_{T}^{\text {track }}$ in a $\Delta R<0.4$ cone around the candidate track

The distributions for these variables are shown in Figs. 3.4 and 3.5 for CC and EC respectively.

These distributions are used to assign probabilities for a given EM object to be signal or background $\S\left(P_{\text {sig }}(\vec{x})\right.$ or $\left.\mathrm{P}_{\mathrm{bkg}}(\tilde{\mathrm{x}})\right)$ where $\vec{x}$ is a vector of the 7 likelihood variables. Given a physics object, each likelihood variable provides a probability for the object to be signal or background, from the normalised distribution of the corresponding variable. Assuming no correlation, these probabilities can be multiplied to give an overall probability for the event:

$$
P(\vec{x})=\prod_{i} P\left(x_{i}\right)
$$

Finally, to distinguish electrons from background objects, the following

\footnotetext{
$\S$ The signal electrons are ones which come from the interaction vertex such as $Z \rightarrow e e$ electrons, $W \rightarrow e \nu_{e}$ electrons. The sources of background electrons are described at the beginning of this section.
} 

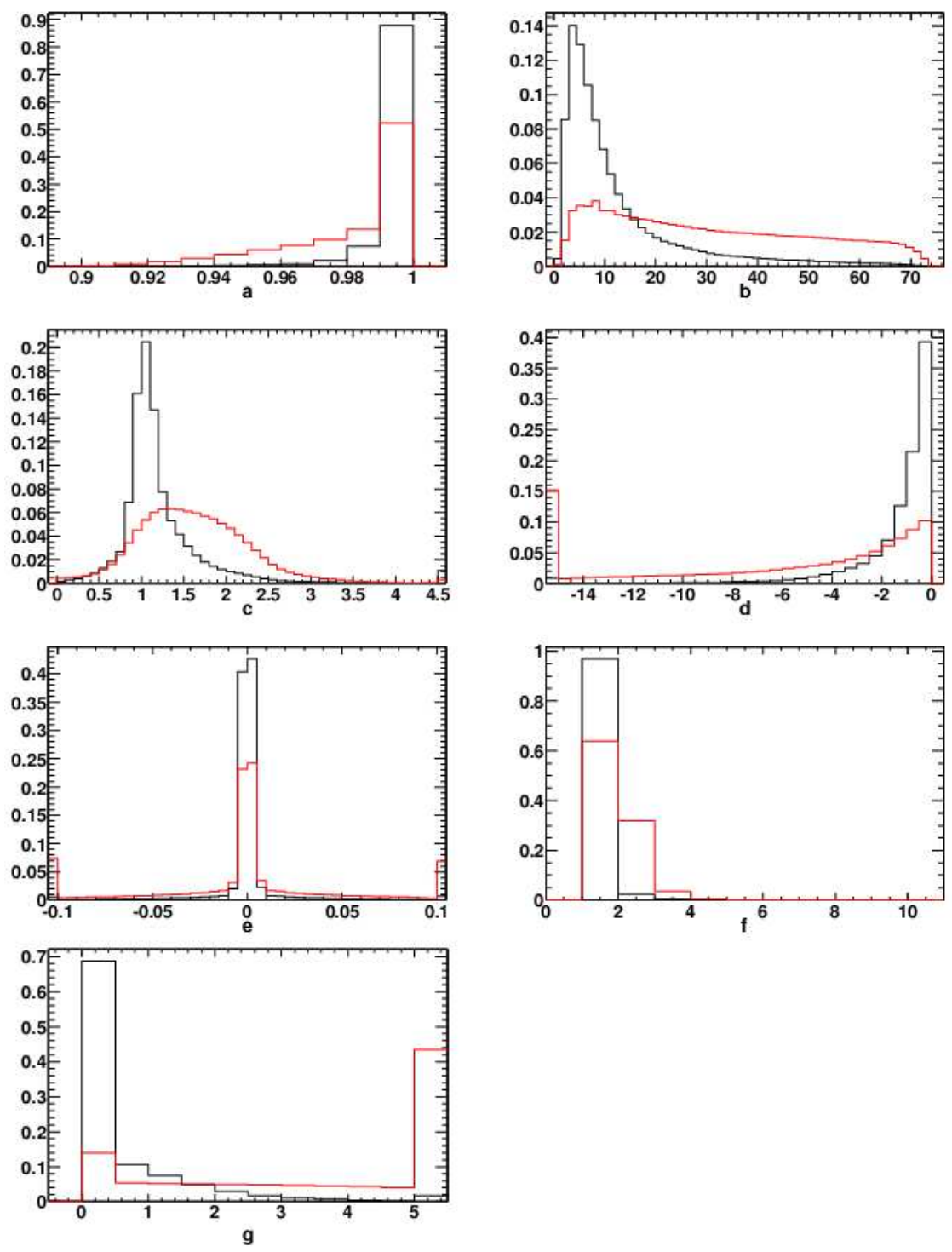

Figure 3.4: The distributions for the input variables to the 7-parameter EM Likelihood in CC - electron (black) and background (red): (a) EM fraction, (b) Hmx7, (c) $E_{T}^{\text {Cal }} / p_{T}^{\text {track }},\left(\right.$ d) Logarithm of $\operatorname{Prob}\left(\chi_{\text {Spatial EM-trk }}^{2}, 2\right)$, (e) Distance of closest approach of the candidate track, (f) Number of tracks in a $\Delta R<0.05$ cone around the candidate track and (g) $\sum p_{T}^{\text {track }}$ in a $\Delta R<0.4$ cone around the candidate track. 

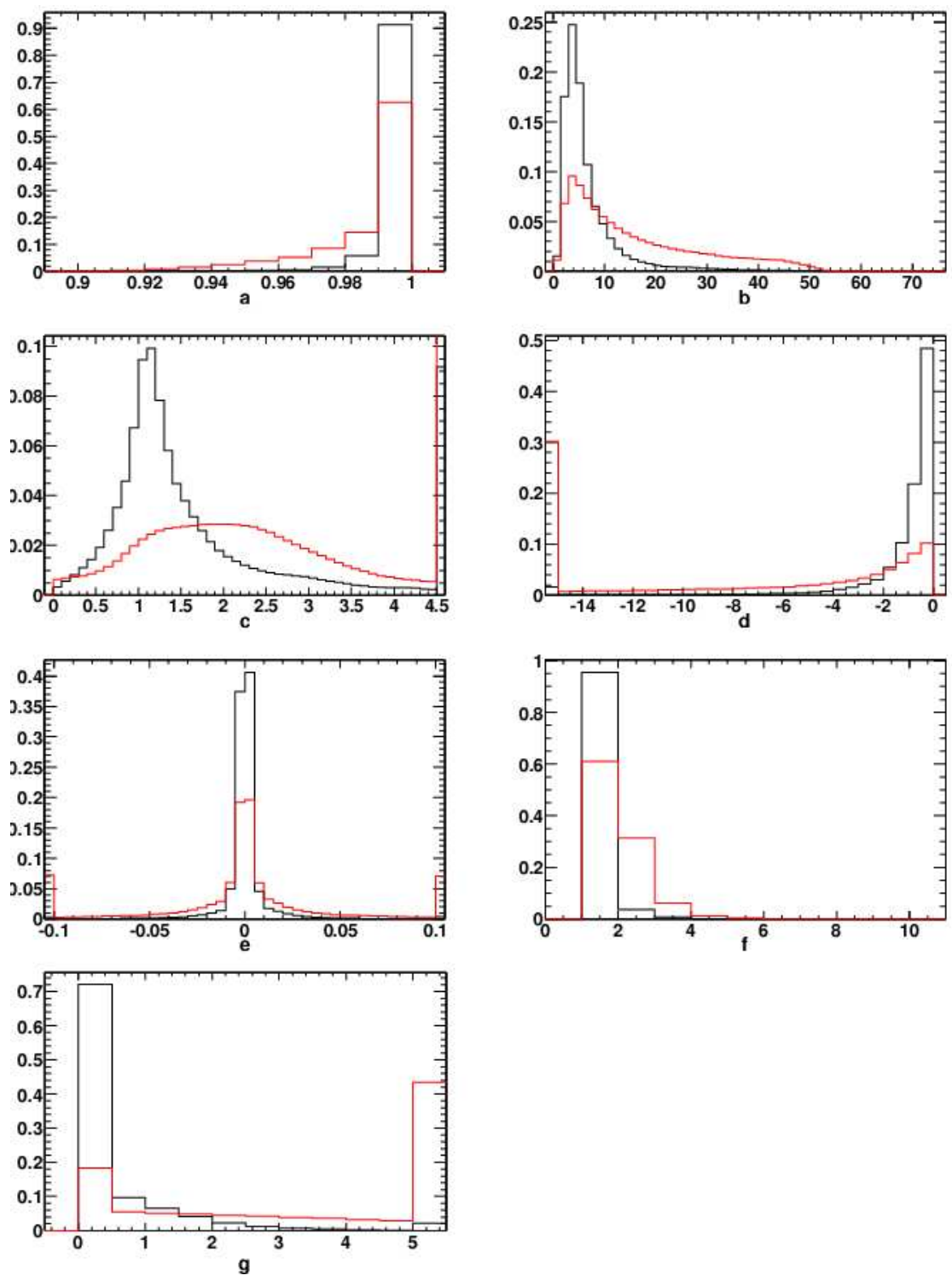

Figure 3.5: The distributions for the input variables to the 7-parameter EM Likelihood in EC - electron (black) and background (red): (a) EM fraction, (b) Hmx7, (c) $E_{T}^{\text {Cal }} / p_{T}^{\text {track }},\left(\right.$ d) Logarithm of $\operatorname{Prob}\left(\chi_{\text {Spatial EM-trk }}^{2}, 2\right)$, (e) Distance of closest approach of the candidate track, (f) Number of tracks in a $\Delta R<0.05$ cone around the candidate track and (g) $\sum p_{T}^{\text {track }}$ in a $\Delta R<0.4$ cone around the candidate track. 
discriminant is used:

$$
\mathcal{L}(\vec{x})=\frac{P_{\text {sig }}(\vec{x})}{P_{\text {sig }}(\vec{x})+P_{\mathrm{bkg}}(\vec{x})}
$$

For electron $\mathcal{L}(\vec{x})$ tends towards 1 whereas, for background objects, $\mathcal{L}(\vec{x})$ tends towards 0 . The background efficiency vs electron efficiency is shown in Figure 3.6 separately for CC and EC. In the present analysis, a cut of $\mathcal{L}(\vec{x})>0.85$ (for both $\mathrm{CC}$ and EC) has been applied which results in $96 \%(91 \%)$ and $26 \%(26 \%)$ signal and background efficiencies for $\mathrm{CC}(\mathrm{EC})$. Detailed documentation on EM Likelihood can be found in Ref. [65].

The electrons are classified into three different categories called "loose", "medium" and "tight". All electrons reconstructed by dØreco, are loose electrons. Of these electrons, the ones which pass the criteria for isolated electro-magnetic clusters i.e., $\mathrm{f}_{\mathrm{EM}}>0.9, \mathrm{f}_{\text {iso }}<0.15$ and $\mathrm{hmx} 7<50$, are medium, while tight electrons are the isolated clusters, which have matching tracks and have passed likelihood criteria. 


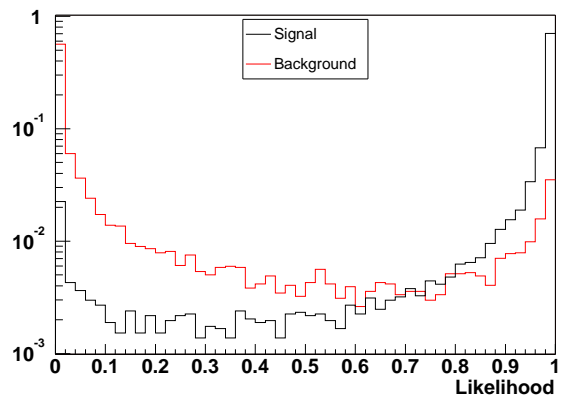

(a)

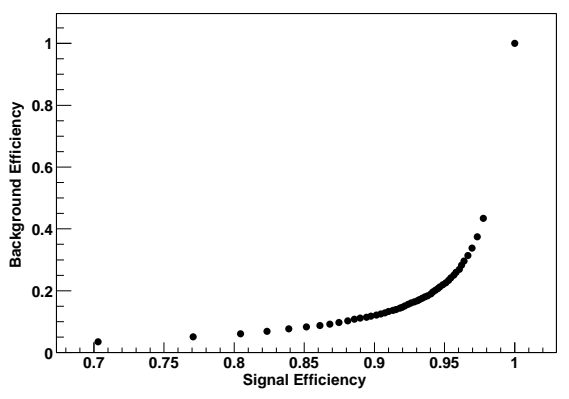

(c)

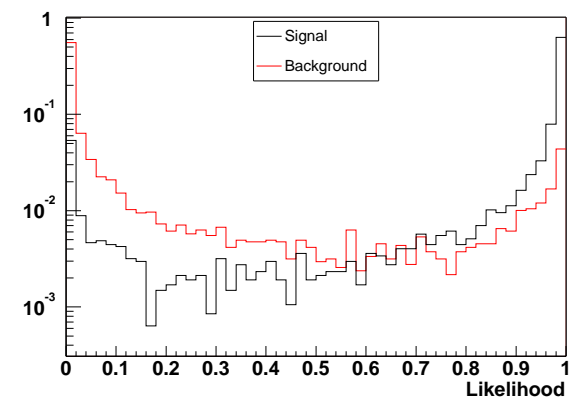

(b)

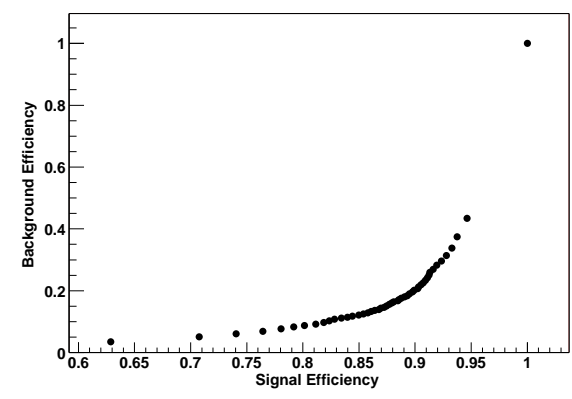

(d)

Figure 3.6: The 7-parameter EM likelihood distributions for electron (black) and background (red) in $\mathrm{CC}(\mathrm{a})$ and $\mathrm{EC}$ (b); the background efficiency vs electron efficiency for different thresholds of EM Likelihood in $\mathrm{CC}(\mathrm{c})$ and $\mathrm{EC}(\mathrm{d})$. 


\subsubsection{Electromagnetic Energy Correction}

The energy of an electron/photon estimated using calorimeter information needs to be corrected because of the differences between the conditions during test beam and DØ experiment. Differences exist in the readout electronics and liquid argon purity, which account for the offset between the test-beam and $D \varnothing$ energy scales. In addition, the energy scales vary from one section to another. These variations are measured using large data samples with electromagnetic clusters, $Z^{0} \rightarrow e^{+} e^{-}$dataset $^{\dagger}$ on module-by-module basis. There are two types of corrections which need to be applied:

\section{Geometrical correction}

The electro-magnetic part of the Central Calorimeter (CC) extends up to pseudo-rapidity 1.1, while two Endcap Calorimeters (north and south, NEC, SEC) extend to $|\eta|<4$. Each of these 3 segments is placed inside the cryostat, surrounding the tracker as well as 2 Tesla solenoid magnet. Moreover, the electromagnetic part of Central Calorimeter with its cylindrical geometry is divided symmetrically into 32 modules in $\phi$ and there are gaps in the intermodule regions, called $\phi$-cracks. Thus in the following situations, the energy

\footnotetext{
${ }^{\dagger} Z^{0} \rightarrow e^{+} e^{-}$data is selected by selecting the events with two good track-matched EM clusters, having opposite sign. A requirement of very low missing $E_{T}$ in the event, is also applied to purify the sample further.
} 
of an electron can not be recovered completely, from the calorimeter cluster.

1. electron loses a significant part of its energy in the tracking apparatus, solenoid and finally in the cryostat.

2. electron moves through the $\eta$ edges (inter-cryostat region) or through the $\phi$-cracks in CC.

These corrections are called "geometrical corrections" as they originate exclusively due the detector geometry.

The loss of energy does not vary with $\phi$ except, around the cracks (0.010.02 radians in azimuthal angle) between the modules of the calorimeter. The variation is expressed as a function of $\phi^{\prime}\left(=\frac{32 \cdot \phi}{2 \pi}-\left[\frac{32 \cdot \phi}{2 \pi}\right]\right) \S$. Fig. 3.7 illustrates this fact where the energy loss is plotted as a function of $\phi^{\prime}+0.1$ (called phimod) to put the crack artificially in the centre of the plot. To quantify further, these losses are $12 \%$ and $30 \%$ for incident electron energy of 250 and $5 \mathrm{GeV}$, respectively.

In order to study the energy loss as a function of $\eta$, the electrons which fall near $\phi$-crack of CC, are removed (electrons reconstructed in region of the

\footnotetext{
$\S[\mathrm{x}]$ are the integer values of $\mathrm{x}$. e.g., $x=0.02,[x]=0.00 ; x=0.98,[x]=0.00 ; x=1.01,[x]=1.00$ etc.
} 


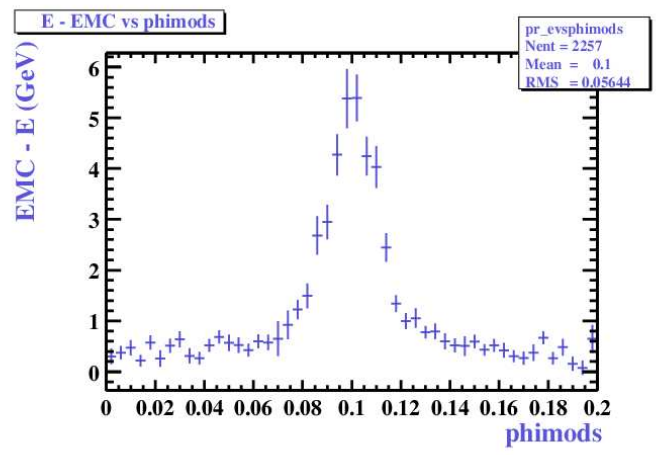

Figure 3.7: Energy loss (shown as, EMC-E) of $20 \mathrm{GeV}$ electron as a function of $\left(\phi^{\prime}+0.1\right)$ (see text); the edge of the module is around 0.1 .

phimmod 0.07-0.13) from the sample of study. The corrected electron energy can be parametrized as

$$
E_{\text {corr }}=E_{\text {reco }}+\operatorname{etacorra}\left(E_{\text {reco }}\right)+\operatorname{etacorrb}\left(E_{\text {reco }}\right) \cdot \eta_{\mathrm{P}}+\operatorname{etacorrc}\left(E_{\text {reco }}\right) \cdot \eta_{\mathrm{P}}^{2},
$$

where the factors etacorra $\left(E_{\text {reco }}\right)$, etacorrb $\left(E_{\text {reco }}\right)$ and etacorrc $\left(E_{\text {reco }}\right)$ are the factors estimated using a single electron Monte Carlo sample. Here $\eta_{\mathrm{P}}$ is defined with respect to the primary vertex of the event. Figure 3.8 shows the fit of energy correction as function of $\eta_{\mathrm{P}}$ as well as the variation of the fit parameters with electron energy. 


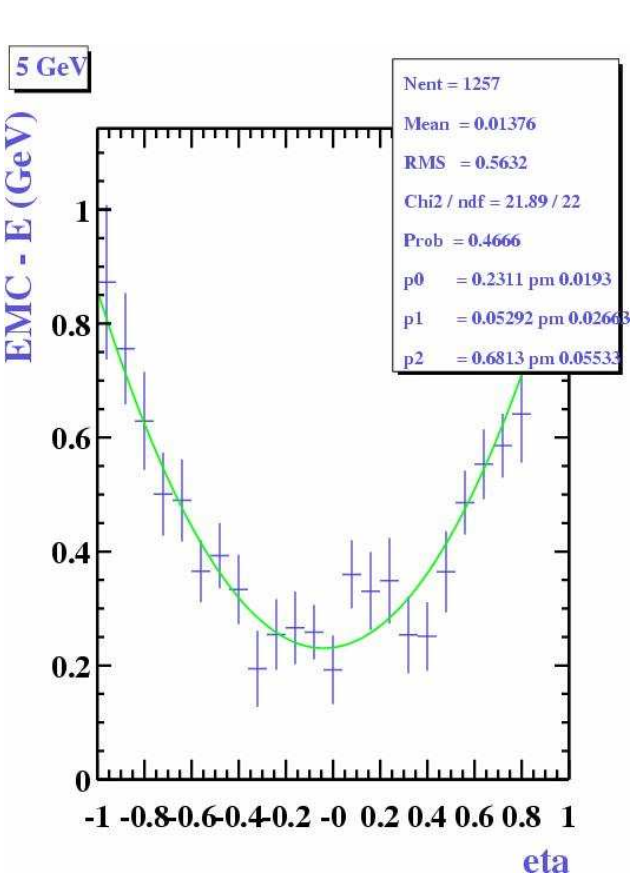

(a)
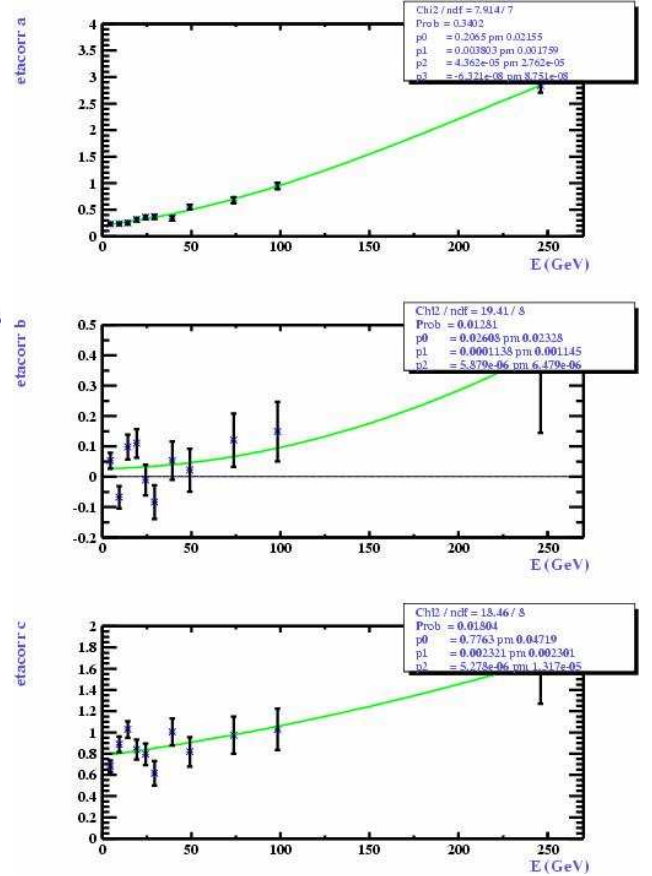

(b)

Figure 3.8: (a) Parabola fit of the energy loss (shown as EMC-E) of $5 \mathrm{GeV}$ electrons as a function of $\eta_{p}$; (b) Variation of the fit parameters, etacorra (top), etacorrb (middle), etacorrc (bottom) as a function of electron energy. 


\section{EM Scale correction}

After geometrical corrections, invariant mass of two reconstructed EM clusters can be written as

$$
\begin{aligned}
\bar{m}_{i}^{2} & =m_{i}^{2} \cdot\left(1+\epsilon^{k}\right) \cdot\left(1+\epsilon^{l}\right) \\
\text { with } \quad \mathrm{m}_{\mathrm{i}}{ }^{2} & =2 \mathrm{E}_{1 \mathrm{i}}^{\mathrm{k}} \cdot \mathrm{E}_{2 \mathrm{i}}^{\mathrm{l}} \cdot\left(1-\cos \theta_{\mathrm{i}}\right),
\end{aligned}
$$

where $E_{1(2) i}^{k / l}$ are the energies of the reconstructed EM clusters 1(2) detected in zones $\mathrm{k}$ and $\mathrm{l}$ respectively for an event $\mathrm{i}$ and $\theta_{i}$ is the angle between the clusters. Here $\epsilon^{\mathbf{k}}, \epsilon^{\mathbf{l}}$ are the correction factors being measured for zones $\mathrm{k}$ and 1 . For each event i, a probability $p_{i}$, that the invariant mass of the EM cluster pair would be $\bar{m}_{i}$ is assigned using a normalized Breit-Wigner:

$$
p_{i}\left(\bar{m}_{i} / m_{Z}\right)=A \cdot \frac{B}{\left(\left(\bar{m}_{i} / m_{Z}\right)^{2}-C\right)^{2}+B} \cdot \frac{1}{\left(\bar{m}_{i} / m_{Z}\right)^{D}},
$$

where $m_{Z}$ is the mass of $Z^{0}\left(=91.2 \mathrm{GeV} / \mathrm{c}^{2}\right)$. The best values of the parameters obtained from Monte Carlo distribution [66], are: $\mathrm{A}=0.1125 \pm 0.0017$, $\mathrm{B}=0.0039 \pm 0.0001, \mathrm{C}=1.035 \pm 0.001$ and $\mathrm{D}=3.0 \pm 2.0$. The minimization of 


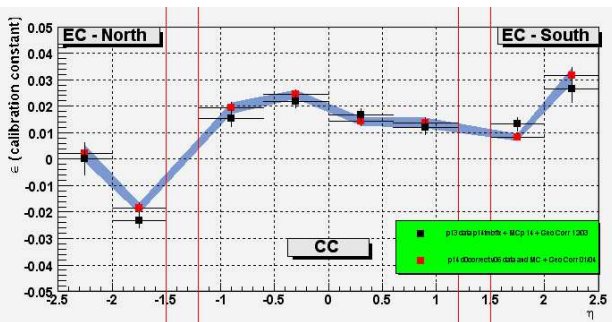

(a)

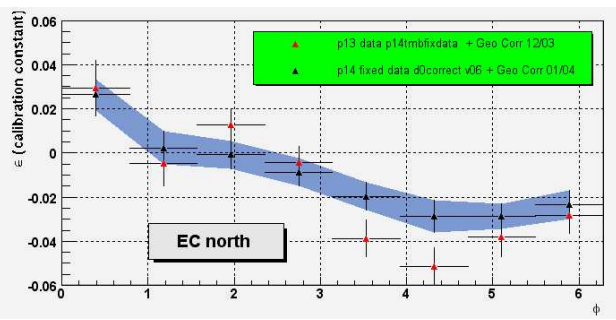

(c)

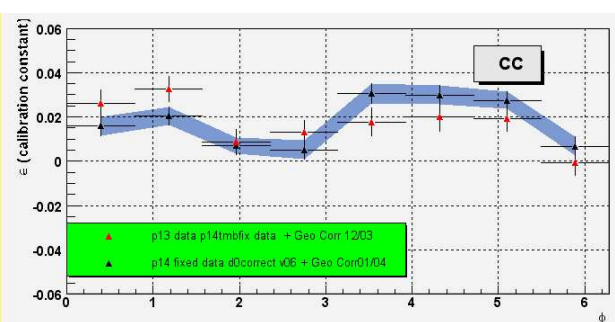

(b)

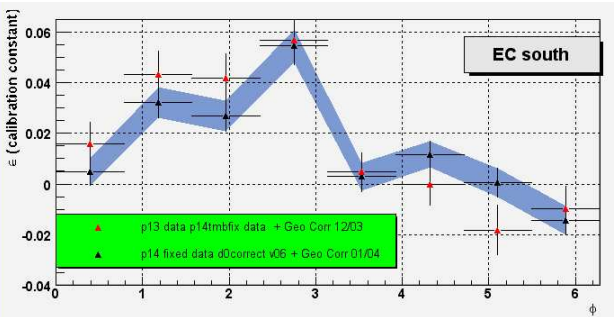

(d)

Figure 3.9: EM energy correction factor $\epsilon$ as functions of (a) $\eta$ in $\mathrm{CC}$, (b) $\phi$ in CC, (c) $\phi$ in North EC and, (d) $\phi$ in South EC (d).

the likelihood

$$
\mathcal{L}\left(\epsilon^{1}, \epsilon^{2}, \ldots\right)=-\sum_{i}^{N^{\text {events }}} \ln p_{i}\left(\frac{m_{i}}{m_{Z}}\right)
$$

for each zone $\mathbf{i}$, provide the correction factor $\epsilon^{i}$. The methodology has been applied for both $\eta$ and $\phi$ calibration. The calibration curves are shown in Fig. 3.9.

This precise calibration of the electromagnetic calorimeter serves as the starting point for calibration of the hadronic energy scale (see section 3.2.5). 
All documentation relevant to the electromagnetic scale calibration can be seen at Ref. [67].

\subsubsection{Muons}

Muons are reconstructed using information from the muon system and the central tracking system. Calorimeter information is only used for a measurement of identification efficiency.

\section{Muon Reconstruction}

In principle, the reconstruction of muon tracks is similar to the reconstruction of tracks in the central tracking system. However, differences in the geometry and electronics of the muon system with those in central tracking system, as well as the need to measure the bend angle (and thus the momentum) of the tracks, require somewhat different algorithms to be used. At first, the muons are identified in the muon system. After grouping the drift chamber and scintillator hits into segments on either side of the toroid (A layer on one side and B/C layers on the other side), tracks are constructed from segments in the B/C layer by searching for matched segments in the A layer. If a segment satisfies loose matching criteria, a more careful fitting is performed to represent a physical path. An estimate of the muon's momentum is obtained 
from the bending angle in the toroidal magnetic field.

A muon, satisfying following quality criteria in the muon system,

- at least 1 wire hit in the A layer

- at least 1 scintillator hit in the A layer

- at least 2 wire hits in the $\mathrm{B} / \mathrm{C}$ layer

- at least 1 scintillator hit in the B/C layer

is called a "local muon". In addition, to reject the cosmic muons, the time difference between scintillator hits in $\mathrm{B}$ or $\mathrm{C}$ layer and in $\mathrm{A}$ layer is required to be $>-10 \mathrm{~ns}$, which is consistent with the muon coming from the interaction point.

\section{Central Track Finding}

Since the momentum resolution of the muon system is quite poor compared to the central tracking system (see Sec. 2.2.7 and 2.2.3, respectively), the local muons are matched to tracks in the central tracking system. In order to find such matches, the muon tracks are extended to the point of closest approach (PCA) to the beam axis i.e., z-axis and the corresponding track parameters are compared with those of candidate central tracks at PCA. A 
global fit is performed with all central tracks within 1 radian in azimuthal and polar angle of a muon track at PCA. The central track with highest $\chi^{2}-$ probability is considered as the track matched to the local muon. If a muon matches to track from central tracking system, the momentum measurement from the muon system is discarded.

\section{Muon Isolation}

Muons coming from leptonic decays of $W$ bosons tend to be isolated from jets and have relatively high transverse momentum $\left(p_{T}\right)$. The main source of background muons is the semileptonic decays of heavy quarks. These muons tend to be non-isolated and have lower transverse momenta. Muon isolation criteria are used to distinguish between muons from these two different sources. The current analysis utilizes information from both calorimeter and central tracking system for this purpose. The muon isolation criteria are based on following two observables:

- Halo(0.1,0.4): Sum of $E_{T}$ of calorimeter clusters in a hollow cone between $\Delta R=0.1$ and $\Delta R=0.4$ away around the muon as schematically shown in Fig. 3.10. In forming this sum, cells in the electromagnetic and fine hadronic calorimeters are used, but not those in the coarse 


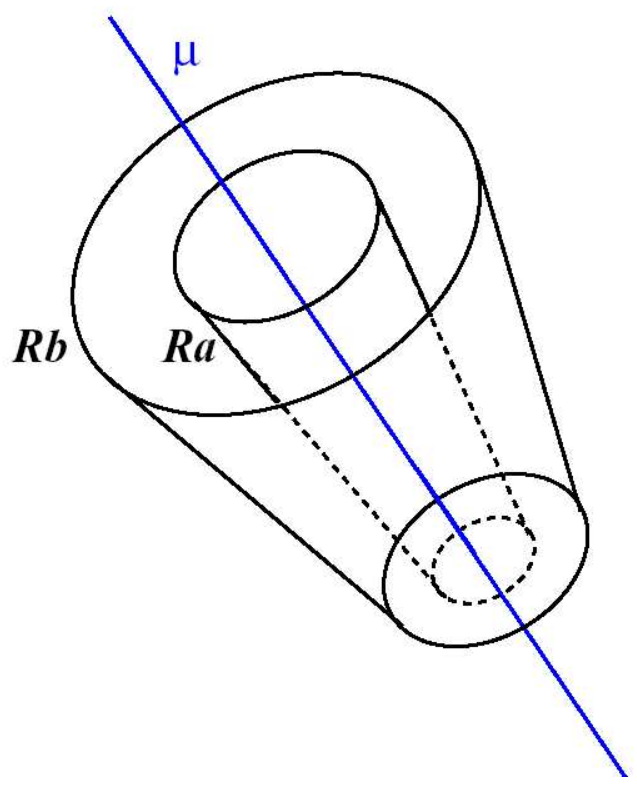

Figure 3.10: A halo cone $\mathrm{R}$, with inner edge $R_{a}$ and outer edge $R_{b}$ surrounding the muon. The transverse energies of all calorimeter cells $\mathrm{i}$ at a radius $R_{i}$ with $R_{b}>R_{i}>R_{a}$ where $R_{a}\left(R_{b}\right)=0.1(0.4)$.

hadronic calorimeter ${ }^{\S}$

- TrkCone(0.5): Sum of the $p_{T}$ of all tracks within a cone of radius $\Delta R=0.5$ surrounding the muon. A track which could be matched to the muon is excluded from this sum.

It has been found that the separation between the two classes of muons mentioned above could be improved by using the difference between their $p_{T}$

\footnotetext{
$\S$ The cells in the coarse hadronic part of the calorimeter are too noisy to be used for measuring muon isolation.
} 
spectra. Thus more powerful isolation variables are defined, taking the ratio of the above mentioned isolation variables and the transverse momentum of the muon, $p_{T \text { muon }}$ :

- $\operatorname{Rat} 11=\operatorname{Halo}(0.1,0.4) / p_{T}^{\text {muon }}$

- $\operatorname{Rattrk}=\operatorname{TrkCone}(0.5) / p_{T}^{\text {muon }}$

The present analysis requires both the variables viz., Rat11 and Rattrk to be $<0.12$ for an isolated muon. Further documentation can be found in Ref. [68].

\section{Muon Promptness}

A prompt muon coming from the decay of a $\mathrm{W}$ boson will have a track originating from the primary vertex of the event. To quantify the association of the muon with the primary vertex, a cut on the DCA significance (see Section 3.1.1 for a definition of DCA) of the central track is applied,

$$
\text { DCA significance }=\frac{\mathrm{dca}}{\sigma_{\mathrm{dca}}}<3
$$

Moreover, a distance $\Delta z(\mu, \mathrm{PV})<1 \mathrm{~cm}$ between the track and the primary vertex is required to further reduce background from cosmics and/or badly 
reconstructed tracks.

\section{Muon Track Quality}

Since the muon momentum is measured in the central tracking system, it is necessary that the track matched to the muon is of good quality. Tracks that fail the selection criteria of $\chi_{\text {track }}^{2} / \mathrm{NDF}<4$ or $\Delta z(\mu, \mathrm{PV})<1 \mathrm{~cm}$ have been observed to have a larger probability to give a very high $p_{T}$ measurement (> $200 \mathrm{GeV})$. Applying these cuts significantly reduces the tail at the high end of the $p_{T}$ distribution of muons. It is believed that the remaining high $p_{T}$ muons are genuine muons, for which the track curvature $q / p_{T}$ is well measured within the finite resolution of the central tracking system.

The present analysis uses two types of muons as defined below. A local muon, i.e., reconstructed in the muon spectrometer is called a "medium" muon if,

- the muon is matched to a track in the central tracking system

- matched central track is of good quality $\left(\chi_{\text {track }}^{2}<4\right)$.

The medium muon definition is extended to a definition of "tight" or "isolated" muon by requiring,

- low DCA significance of the matched central track $\left(|D C A| / \sigma_{D C A}<3\right)$, 
- compatibility of the central matched track with the primary vertex of the event $(|\Delta \mathrm{z}(\mu, \mathrm{PV})|<1 \mathrm{~cm})$,

- isolation from unclustered calorimeter energy

$$
\left(\operatorname{Rat} 11=\operatorname{Halo}(0.1,0.4) / p_{\text {Tmuon }}<0.12\right),
$$

- isolation from other tracks $\left(\right.$ Rattrk $\left.=\operatorname{TrkCone}(0.5) / p_{\text {Tmuon }}<0.12\right)$.

\subsubsection{Jets}

Jets are produced either from quarks or from gluons. Various levels at which jets can be defined are illustrated in Fig. 3.11. Parton jets are just the partons in the fixed order of perturbative QCD calculations while a Particle jet is the set of hadrons produced by a parton after hadronisation. The hadron jets deposit energy in the calorimeter cells and can be reconstructed by summing up the cell energy.

\section{Jet Reconstruction}

When a parton is produced in a $p \bar{p}$ collision, the parton is expected to fragment and produces a number of hadrons, which travel approximately in the same direction as the original parton. If one of the original partons radiates a gluon prior to the fragmentation process, the emitted gluon will then 


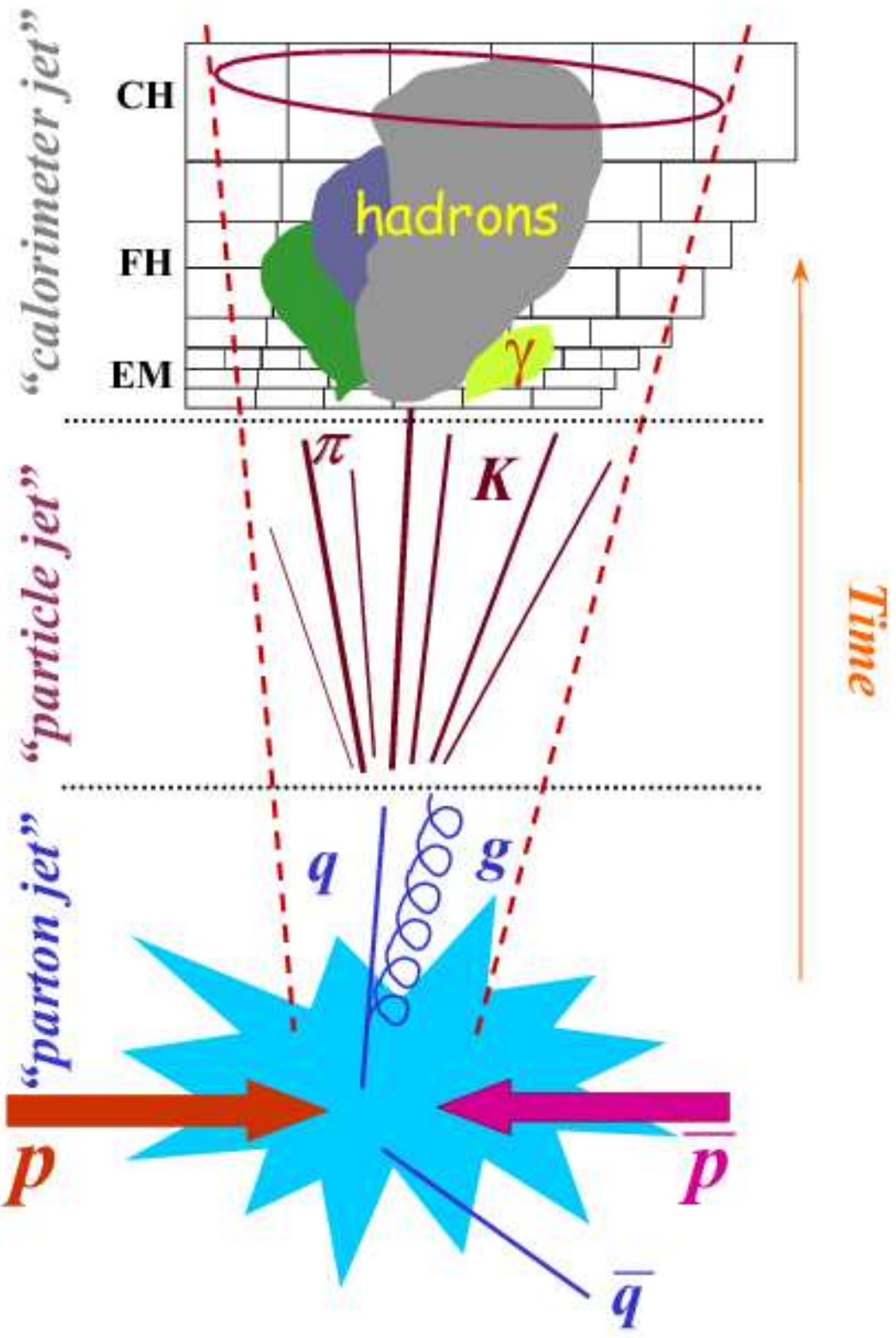

Figure 3.11: An illustration of the different levels of jet definition. 
fragment to produce another spray of hadrons. If the gluon is emitted at a small angle with respect to the original parton direction, these particles will tend to fall in the same or nearby calorimeter cells as the hadrons from the initial parton, and will still be seen as a single cluster. One might be able to distinguish these two clusters if the calorimeter has fine enough granularity. Even then, if the gluon is emitted at a small enough angle, the clusters will look like a single jet. In order to handle this kind of problems along with several other attributes of a jet, a detailed prescription ("Jet Algorithm") is required to reconstruct the jets. Historically, Cone Algorithm has been the jet algorithm of choice for hadron-hadron colliders. The original Snowmass cone algorithm [69], which was used by DØ during Tevatron Run I, reconstructs by definition massless jets. However, in Tevatron Run II based on the recommendations of Run II jet physics group [70], DØ has decided to use a modified version of cone algorithm where the jets are no longer massless, as described below.

\section{DØ Run II Cone Algorithm}

The Cone algorithm forms jets by collecting together the calorimeter towers, whose centres lie within a circle of a specific radius $\mathrm{R}$ in $y \times \phi$ space $^{\dagger}$. The

\footnotetext{
${ }^{\dagger} \mathrm{y}$ is the true rapidity.
} 
kinematic parameters of a jet are estimated, by combining the parameters of the contributing towers, through a certain procedure, called "recombination scheme". Starting with a trial geometric centre (or axis) for a cone in $y \times$ $\phi$ space, the energy-weighted centroid is calculated including contributions from all towers within the cone. If the geometric axis is not the same as the energy-weighted axis, this new axis in $y \times \phi$ is used as the geometric centre for a new trial cone. As this calculation is iterated, the cone centre "flows" until a "stable" solution is found i.e., the energy-weighted centroid is aligned with the geometric axis of the cone.

In order to reconstruct jets, following steps are executed on a list of towers, specified with the 4 -vectors $\left(\mathrm{E}_{\text {tower }}, \tilde{\mathrm{p}}_{\text {tower }}\right)$ with coordinates $\left(\phi_{\text {tower }}, \mathrm{y}_{\text {tower }}\right.$ $\left.=\frac{1}{2} \ln \frac{E_{\text {tower }}+p_{z, t \text { tower }}}{E_{\text {tower }}-p_{z, \text { tower }}}\right)$, with respect to primary vertex.

1. The calorimeter towers with transverse energy larger than $0.5 \mathrm{GeV}$ are taken to be the starting seeds of the algorithm. A list of towers is made by ordering the towers according to their $E_{T}$ 's.

2. Starting with the highest $E_{T}$ tower, a stable cone of radius $\mathrm{R}=0.5^{\S}$ is searched for in $y \times \phi$ space. For a specific geometric centre of the cone

\footnotetext{
$\S^{\S} \emptyset$ reconstructs jets of various cone sizes, viz., $0.3,0.5,0.7$; the present analysis uses the cone size of 0.5
} 
$\left(y^{C}, \phi^{C}\right)$ having a radius $\mathrm{R}$, a tower, $i$ within the cone satisfies

$$
\mathrm{i} \subset \mathrm{C}: \sqrt{\left(\mathrm{y}_{\text {tower }}^{\mathrm{i}}-\eta^{\mathrm{C}}\right)^{2}+\left(\phi_{\text {tower }}^{\mathrm{i}}-\phi^{\mathrm{C}}\right)^{2}} \leq \mathrm{R}
$$

while the definition of a stable cone is,

$$
\mathrm{y}^{\mathrm{C}}=\frac{\sum_{\mathrm{i} \subset \mathrm{C}} \mathrm{E}_{\mathrm{T}, \text { tower }}^{\mathrm{i}} \mathrm{y}_{\text {tower }}^{\mathrm{i}}}{\mathrm{E}_{\mathrm{T}}^{\mathrm{C}}}, \phi^{\mathrm{C}}=\frac{\sum_{\mathrm{i} \subset \mathrm{C}} \mathrm{E}_{\mathrm{T}, \text { tower }}^{\mathrm{i}} \phi_{\text {tower }}^{\mathrm{i}}}{\mathrm{E}_{\mathrm{T}}^{\mathrm{C}}}
$$

with

$$
\mathrm{E}_{\mathrm{T}}^{\mathrm{C}}=\sum_{\mathrm{i} \subset \mathrm{C}} \mathrm{E}_{\mathrm{T}, \text { tower }}^{\mathrm{i}}
$$

The jet 4-momentum $P_{\text {jet }}$ is just a sum of the 4-momentum of the associated towers

$$
P_{\text {jet }} \equiv\left(E_{\text {jet }}, \vec{p}_{\text {jet }}\right)=\sum_{\text {tower }} P_{\text {tower }},
$$

3. The process is repeated for all seed towers and a list of jets is obtained. Jets with transverse momenta smaller than $8 \mathrm{GeV}$ are removed from the list.

4. Each jet is then compared to the others in the list to determine if it shares towers with any others. If there are any overlaps, the $E_{T}$ 's from 
all the shared towers are added, and compared to the $E_{T}$ of the softer jet. If the shared $E_{T}$ is greater than half of the $E_{T}$ of the softer jet, the jets are merged into one object. Otherwise, they are split into two jets, with each tower being assigned to the jet with nearest center. In either case, the jet axis is recalculated one last time, including all the appropriate towers without any further iterations.

5. The calorimeter energy of the jets are corrected using Jet Energy Scale (JES) described later in Sec. 3.2.5. The energy of a jet that is used in this analysis, is the JES corrected energy.

\section{Selection Criteria}

Once the jets are reconstructed, further offline cuts are applied to improve quality of the jets and reject the noise jets.

- In order to remove the isolated electromagnetic particles, a cut on the fraction of energy deposited in the electromagnetic section of the calorimeter $(\mathrm{EMF})$ is applied $(0.05<E M F<0.95)$.

- To remove the jets which predominantly deposit their energy in the Coarse Hadronic section of the calorimeter, a cut on the fraction of energy deposited therein $(\mathrm{CHF})$ is applied $(\mathrm{CHF}<0.4)$. This particu- 
lar cut removes the possible inclusion of noise in the coarse hadronic section, which is much more noisy than the rest of the calorimeter.

- The jet can include a hot cell in the calorimeter and thus the jet $E_{T}$ can get mis-measured. To reduce this effect, a cut on the ratio of $E_{T}$ 's of the highest to next-to-highest energy cell in a jet (HotF) is applied $(\operatorname{HotF}<10)$.

- A jet from a hadron usually deposits its energy in more than one tower. To ensure this as well as to reduce the possible contamination from a single hot tower, a cut on the number of towers containing $90 \%$ of the jet energy (n90) is required (n90>1).

- To reduce the effect of noise in jet reconstruction, the $E_{T}$ of the reconstructed jets is compared with just the raw sum from the calorimeter towers, contributing to the jet, i.e.,

$$
\begin{aligned}
\frac{\sum_{\text {tower }} E_{T}^{\text {tower }}}{E_{T}^{\text {jet }} \cdot(1-C H F)} & >0.4(\mathrm{CC} \text { or EC }) \\
& >0.2(\text { ICR } \text { i.e., } 0.8<|\eta|<1.5)
\end{aligned}
$$




\subsubsection{Jet Energy Calibration}

Before making any comparison between experimental data and theory, the jet energy as measured by the $\mathrm{D} \varnothing$ calorimeter, must be corrected to get back the original energy of the jet which went into the detector. This is called the Jet Energy Scale (JES) correction. The jet energy $E_{\text {det }}$, as reconstructed from the calorimeter using cone algorithm, is corrected to the particle level $E_{\text {part }}$ according to the following formula

$$
E_{\text {part }}\left(E_{\mathrm{det}}, \eta, R, \mathcal{L}\right)=\frac{E_{\mathrm{det}}-\mathcal{O}(R, \eta, \mathcal{L})}{\mathcal{R}_{\mathrm{jet}}\left(E_{\mathrm{det}}, \eta, R\right) \mathcal{S}\left(R, \eta, E_{\mathrm{det}}\right)}
$$

where $\eta, \mathrm{R}$, and $\mathcal{L}$ are jet pseudo-rapidity, the cone radius, and the instantaneous luminosity respectively. The correction consists of three components, $\mathcal{O}, \mathcal{R}_{\text {jet }}$, and $\mathcal{S}$, which are discussed below.

\section{Offset $\mathcal{O}(R, \eta, \mathcal{L})$}

Offset in jet energy scale determination is defined as the energy which is not associated with hard scattering. Contributions to offset originate from electronic noise, Uranium noise, pile-up from the previous beam crossing and the energy from additional minimum bias interactions in the same beam crossing. Signal shaping time for the $\mathrm{D} \varnothing$ calorimeter preamplifier is longer 
than the bunch crossing time; so it is possible that before the signal from an earlier bunch crossing dies, a new signal is formed and it sits over the tail of the previous signal. This causes an improper determination of base-line to be subtracted electronically from the signal we are interested in. This effect is called pile-up. This depends on the instantaneous luminosities of previous bunches, and also on the location of present bunch in the super-bunch, as the bunches are not spaced uniformly in time inside a super-bunch. For instance if the bunch where the present event occurred is at the beginning of a super bunch, then base-line will be much less affected as compared to when the bunch is in the middle or at the end of a super-bunch. If $\left\langle N_{\mathrm{MB}}\right\rangle$ is the average number of minimum bias interactions per bunch crossing (varies widely with the instantaneous beam luminosity, $\mathcal{L}$ ), the effective number of interactions in a given bunch crossing where one hard scattering has occurred, becomes $\langle N\rangle=1+\left\langle N_{\mathrm{MB}}>\right.$. The total offset correction is then written as

$$
\begin{aligned}
\mathcal{O} & =E_{\mathrm{ue}}\left(1+<N_{\mathrm{MB}}>\right)+E_{\text {noise }}+E_{\text {pileup }} \\
& =E_{\mathrm{ue}}+<N_{\mathrm{MB}}>\cdot E_{\mathrm{ue}}+E_{\text {noise }}+E_{\text {pileup }} \\
& =E_{u e}+E_{\Theta},
\end{aligned}
$$


where $E_{\mathrm{ue}}$ is the energy associated with the underlying event, $E_{\text {noise }}$ is the energy associated with the electronic noise and uranium noise and $E_{\text {pileup }}$ is the energy associated with pileup. In $\mathrm{D} \varnothing$ the offset correction factors viz., $E_{\text {ue }}$ and $E_{\Theta}$ are measured separately using the minimum bias events for both data and Monte Carlo.

Response $\mathcal{R}_{\text {jet }}\left(E_{\text {det }}, \eta, R\right)$

Energy measured by a calorimeter, depends on the type and energy of a particle, passing through the calorimeter. The individual particles in a jet are usually of much lower energies than the energy of the jet. Therefore, they get affected by the non-linear energy response of the $\mathrm{D} \varnothing$ calorimeter $\mathcal{R}_{\text {jet }}$ is the correction factor to take care of such differences in the response of the calorimeter. Moreover, the calorimeter response is also affected by the presence of dead material in front of the calorimeter. The correction factor, $\mathcal{R}_{\text {jet }}$, is measured from the $p_{T}$ imbalance in $\gamma+$ jet events. Ideally, for a $\gamma+$ jet event, the photon should be back-to-back with the jet in the transverse plane, i.e.,

$$
\vec{p}_{T \gamma}+\vec{p}_{T \text { jet }}=0
$$


However, due to different response of calorimeter to photons, $R_{\mathrm{em}}$ and hadrons, $R_{\text {jet }}$, one would observe an overall imbalance in transverse momenta:

$$
R_{\mathrm{em}}{\overrightarrow{p_{T \gamma}}}_{T}+R_{\text {jet }} \vec{p}_{T \text { jet }}=-\overrightarrow{\mathscr{E}_{T}} \quad \text { or } \quad R_{\mathrm{em}}{\overrightarrow{p_{T \gamma}}}-R_{\text {jet }} \vec{p}_{T \gamma}=-\overrightarrow{\mathscr{E}_{T}}
$$

as $\vec{p}_{T \text { jet }}=-\vec{p}_{T \gamma}$. This can be rewritten in terms of missing $E_{T}$ corrected for EM response ${ }^{\dagger}$ of the calorimeter

$$
{\overrightarrow{E_{T}}}^{\text {corr }}={\overrightarrow{E_{T}}}_{-} \vec{p}_{T \gamma}+R_{\mathrm{em}} \vec{p}_{T \gamma}
$$

From the Eqs. 3.25 and 3.26 , the $R_{\text {jet }}$ is given by

$$
R_{\text {jet }}=1+\frac{{\overrightarrow{E_{T}}}^{\text {corr }} \cdot \vec{n}_{T \gamma}}{p_{T \gamma}}
$$

with $\vec{n}_{T \gamma}=\vec{p}_{T \gamma} / p_{T \gamma}$. These response corrections are estimated for both data and Monte Carlo with $\gamma+$ jet events in different regions of the calorimeter.

\footnotetext{
${ }^{\dagger}$ Electromagnetic scale $R_{\mathrm{em}}$ is determined from matching the $\mathrm{e}^{+} \mathrm{e}^{-}$invariant mass peak in $\mathrm{Z} \rightarrow \mathrm{e}^{+} \mathrm{e}^{-}$events to the known $\mathrm{Z}$ boson mass. Since electrons and photons are identical in terms of calorimeter objects, the electromagnetic scale derived using electron, is applicable for the energy correction of a photon.
} 
Showering $S\left(R, \eta, E_{\mathrm{det}}\right)$

Showering correction is due to the energy that leaks out of the fixed radius cone during the shower development, while a particle passes through the calorimeter. A significant part of the particle's energy may fall outside the cone because of the magnetic field, interaction with the dead material in front of the calorimeter or simply because of the interaction with the calorimeter material itself. This correction is strongly dependent on the size of the cone and increases with the jet rapidity as the forward cones have smaller absolute size (in the units of length). This correction has been derived by studying the shower profiles of the jets in $\gamma+$ jets events.

Since the beginning of Run II, D $\varnothing$ has been trying to improve the techniques for JES determination and numerous versions of JES have been released till the date. The current analysis uses the v5.1 version of JES, details of which can be found in Ref. [71]. Fig. 3.12 (Fig. 3.14) shows the total JES correction and the uncertainties associated with JES as functions of jet $E_{T}$ and jet $\eta$ for data (Monte Carlo). Fig. 3.13 shows the uncertainties associated with individual correction factors of JES (response, offset and showering) as functions of jet $E_{T}$ and jet $\eta$ for data. 

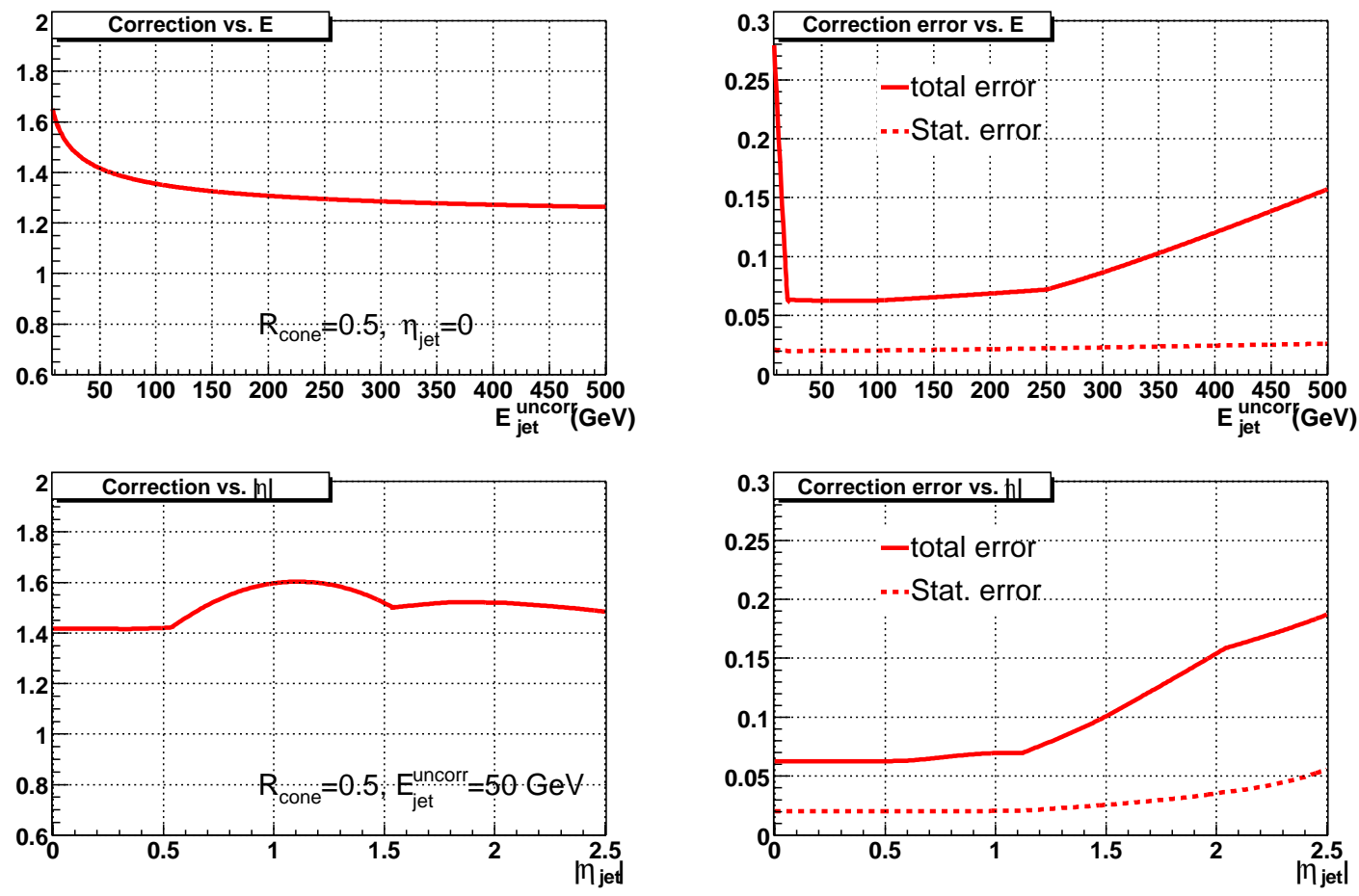

Figure 3.12: The total jet energy scale correction and its uncertainty for data, as a function of uncorrected jet energy for a jet at $\eta=0.0$ (top) and as a function of jet $\eta$ for a jet of $E_{\text {jet }}=50.0 \mathrm{GeV}$ (bottom). The displayed uncertainties are statistical and total (statistical and systematic errors are added in quadrature). 

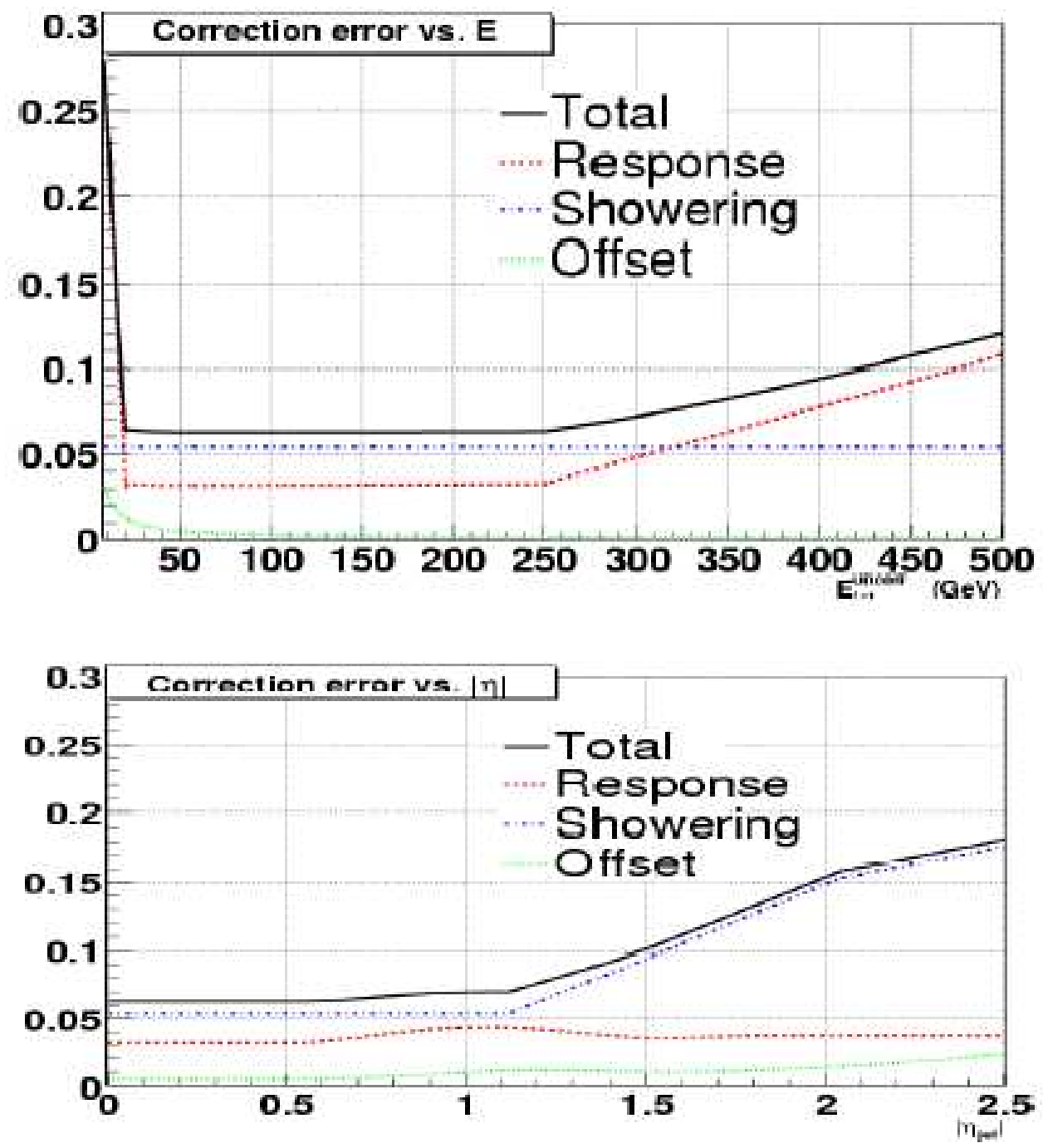

Figure 3.13: The uncertainties are displayed separately for the offset, response and showering corrections, and for the total correction as a function of $E_{T}$ (top) and $\eta$ (bottom) of the jets (in each case, statistical and systematic errors are added in quadrature). 

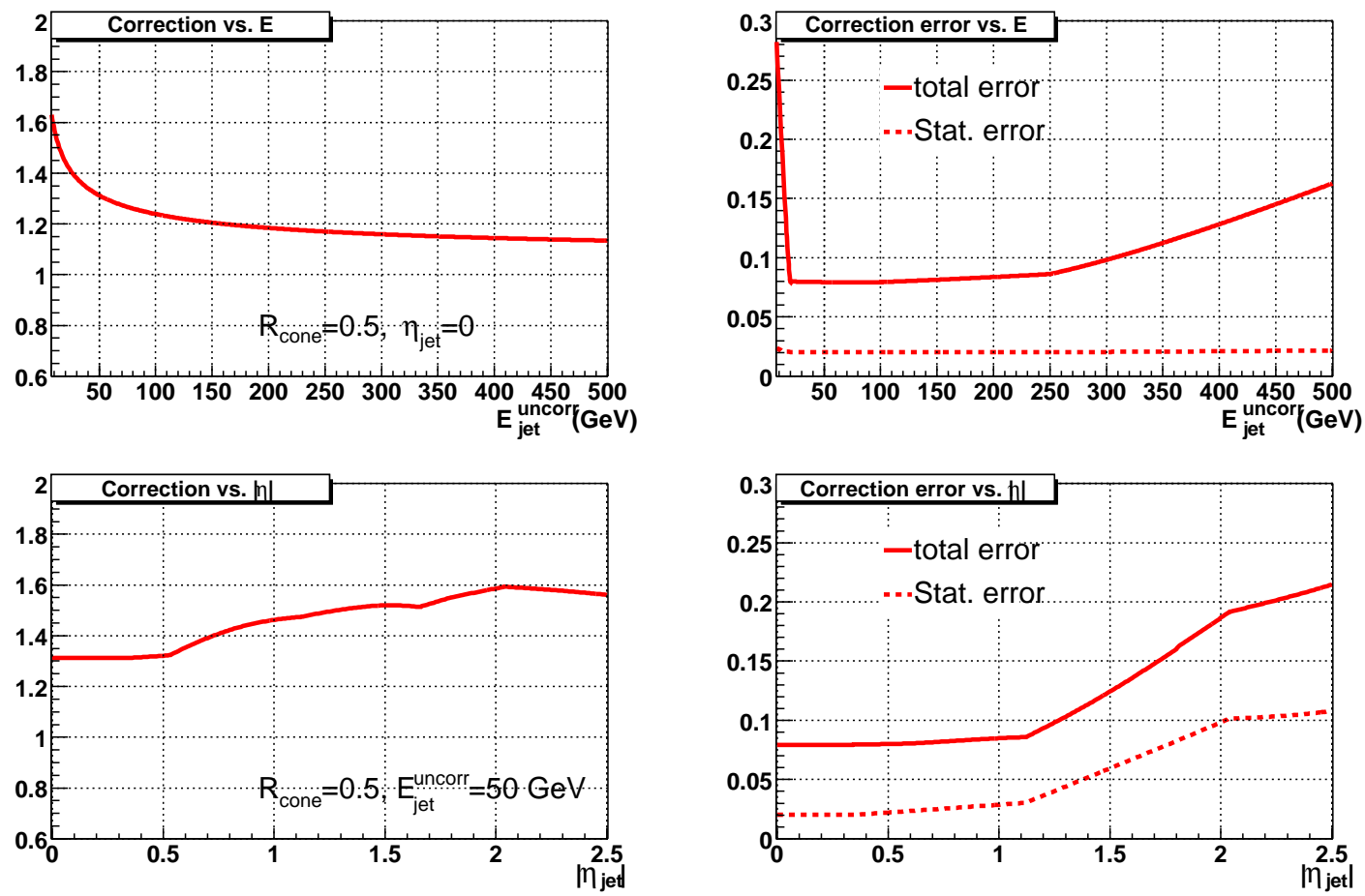

Figure 3.14: The total jet energy scale correction and its uncertainty for Monte Carlo, as a function of uncorrected jet energy for a jet at $\eta=0.0$ (top) and as a function of jet $\eta$ for a jet of $E_{\text {jet }}=50.0 \mathrm{GeV}$ (bottom). The displayed uncertainties are statistical and total (statistical and systematic errors are added in quadrature). 


\subsubsection{Missing $E_{T}$}

From momentum conservation and the fact that the colliding proton and anti-proton have opposite momenta, it follows that the total vector sum of the momenta of all final-state particles in an event must be zero. However, one can not apply total momentum conservation in the longitudinal direction to the beam, as many particles escape detection by going down the beam pipe. On the other hand, in the transverse direction to the beam all the particles except the neutrinos are expected to be detected. Thus one can apply the momentum conservation in the plane transverse to the beam direction. An imbalance in the transverse energy of an event as a whole, indicates the presence of neutrinos. Such an imbalance is known as "missing $E_{T}$ " in the event, denoted by $E_{T}$. This quantity is determined by summing the transverse energy components of every calorimeter and ICD cell:

$$
\begin{aligned}
& E_{x}^{c a l}=-\sum_{i=1}^{N_{\text {cells }}} E_{i} \sin \left(\theta_{i}\right) \cos \left(\phi_{i}\right)-\sum_{j} \Delta E_{x}^{j}, \\
& E_{y}^{\text {cal }}=-\sum_{i=1}^{N_{\text {cells }}} E_{i} \sin \left(\theta_{i}\right) \sin \left(\phi_{i}\right)-\sum_{j} \Delta E_{y}^{j} .
\end{aligned}
$$

The second term in the equations above is a sum over the corrections in $E_{T}$ applied to all electrons and jets in the event. The magnitude of $\mathbb{E}_{T}^{\mathrm{cal}}$ is 
obtained by summing the $x$ and $y$ components in quadrature i.e., $\not_{T}^{\text {cal }}{ }^{2}=$ $E_{x}^{\mathrm{cal}^{2}}+E_{y}^{\mathrm{cal}}{ }^{2}$. The Eqs. 3.28 are used to estimate the $E_{T}\left(=-E_{T}^{\mathrm{cal}}\right)$ of an event without muons. Since muons deposit a small portion of their energy in the calorimeter, the $\vec{E}_{T}$ of the event, is recomputed by subtracting the transverse momenta of all muons in the event

$$
\begin{aligned}
& E_{x}=E_{x}^{\mathrm{cal}}-\sum_{i} p_{x}^{\mu_{i}} \\
& \#_{y}=E_{y}^{\mathrm{cal}}-\sum_{i} p_{y}^{\mu_{i}}
\end{aligned}
$$

This represents the total transverse energy carried away by particles which do not interact in the calorimeter or just the "missing $E_{T}$ " in an event. 


\section{Chapter 4}

\section{Data and Monte Carlo}

Run II of Tevatron has started in spring of 2001. Large volumes of dataset are being recorded by the $\mathrm{D} \varnothing$ experiment. The present analysis is based on the dataset collected between June 2002 and April 2004. This chapter describes the details of the dataset, and performance of the trigger used in the present analysis. The Monte Carlo event simulations for signal and background processes are also presented in this chapter.

\subsection{Data Sample}

Since the beginning of Run II, DØ experiment has encountered several challenges, while commissioning various detector parts and calibrating them. The 
implementation of desired trigger conditions required several tests, leading to a significant down-time in data recording. This analysis is based on a dataset which is recorded by the $\mathrm{D} \varnothing$ experiment after the commissioning phase.

\subsubsection{Integrated Luminosity}

The beam luminosity represents the rate of $p \bar{p}$ collisions per unit area. At $\mathrm{D} \varnothing$ the beam luminosity is measured using the luminosity monitors (LM) described in Sec. 2.2.2 and is based on the fact that the number of interactions in a $p \bar{p}$ bunch crossing follows Poisson hypothesis. Whenever an interaction occurs, the remnants of the incident proton and anti-proton give a pair of time correlated hits in the luminosity counters. The difference in their arrival times at the luminosity counters, gives a quick measurement of the $z$ position of interaction with a precision of $\sim 6 \mathrm{~cm}$. Scalers record the number of time correlated pairs of hits during the $p \bar{p}$ collisions and the delivered beam luminosity is defined as

$$
\mathcal{L}_{\text {beam }}=-\frac{\mathrm{f} / 159}{\sigma} \sum_{\mathrm{i}=1}^{159} \ln \left(1-\frac{\Delta \mathrm{LM}_{\mathrm{i}}}{\Delta \text { ticks } 159}\right)
$$

where $f$ is the revolution frequency of a p or $\bar{p}$ bunch in the Tevatron, $\sigma$ is the effective $p \bar{p}$ cross section (including acceptance and detection efficiency 
of LM), $\Delta \mathrm{LM}_{\mathrm{i}}$ is the scaler count from the LM for $\operatorname{tick}^{\S} i$, and $\Delta$ ticks is the length of the luminosity block ${ }^{\dagger}$ in units of 132 ns ticks. It should be noted that the delivered beam luminosity is calculated independently for each of the 159 ticks and is summed [72] to obtain the average instantaneous luminosity. The integrated luminosity is the integral of $\mathcal{L}$ over a period of time i.e., $\int \mathcal{L} \cdot d t$ and represents the volume of a dataset and is typically expressed in $\mathrm{pb}^{-1}\left(=10^{-36} \mathrm{~cm}^{-2}\right)$. Fig. 4.1 shows the integrated luminosity of the data recorded by the DØ experiment during Run II. There could be hardware failures in different parts of the detector, e.g., failure of a power supply unit, leading to periods in data recording. The integrated luminosity after exclusion of such down-time, is called the "recorded luminosity" as indicated in Fig. 4.1. Further details regarding D $\varnothing$ luminosity measurement can be found in Ref. [37].

\footnotetext{
$\S$ As mentioned in Sec. 2.1, the bunches of protons or anti-protons are not spaced uniformly in time inside the Tevatron ring. They are separated by a smallest unit of time, called "tick". Each tick is of $132 \mathrm{~ns}$ in time while there are 159 ticks in total; $p \bar{p}$ collisions occur only in 36 ticks.

${ }^{\dagger}$ The scalers are read out once in a minute and a unique identifier called Luminosity Block Number (LBN) is attributed to the data that is read out.
} 


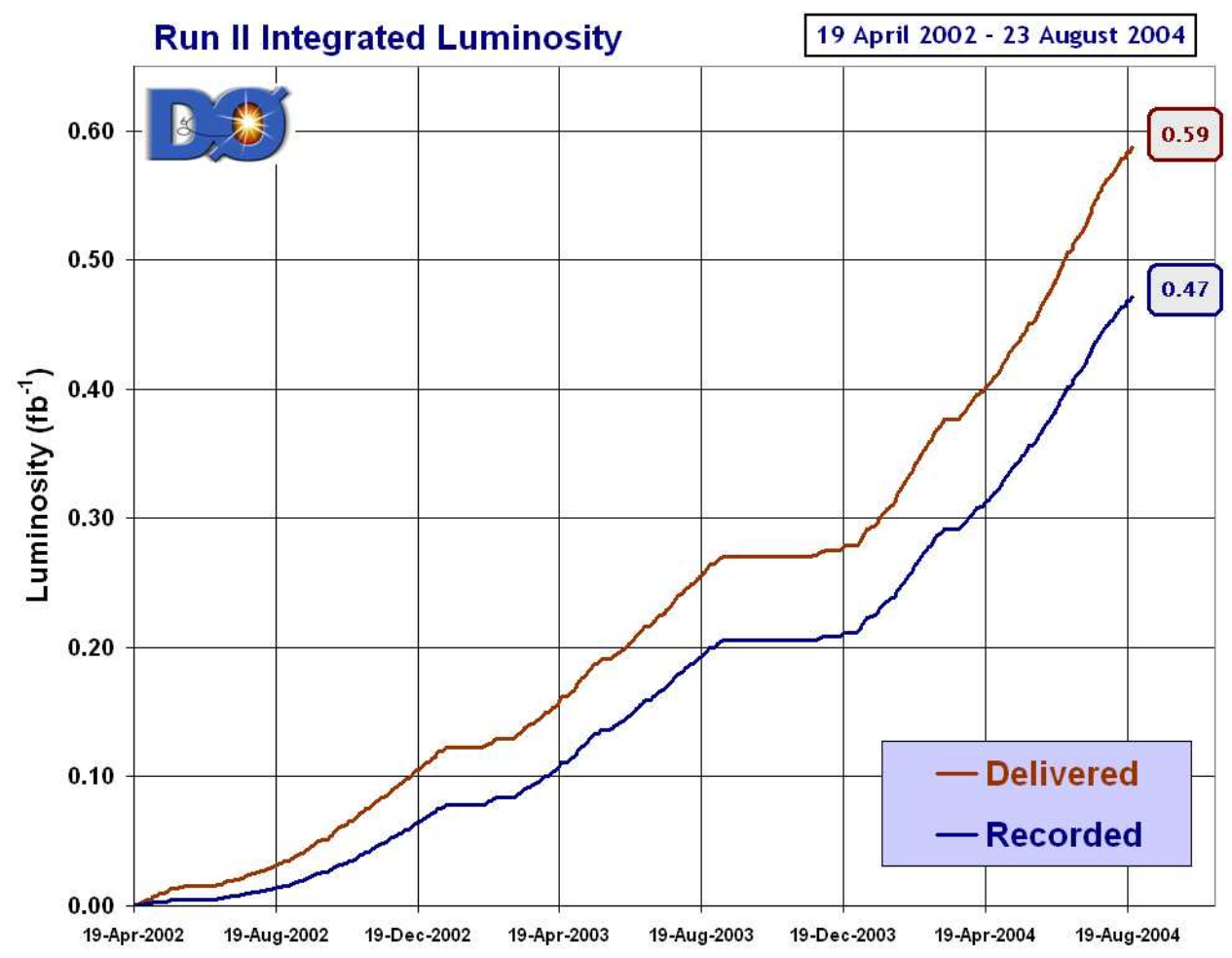

Figure 4.1: The integrated luminosity for Tevatron Run II from April 2002 to August $2004 \mathrm{in} \mathrm{pb}^{-1}$. 


\subsubsection{Data Quality}

The data quality can fluctuate due to several reasons. Minor hardware problems in an unit of a detector component without any interruption in data recording, would result in strange distributions of kinematic variables. To illustrate further, if there is a hardware problem in one of the calorimeter read out units, the missing transverse energy $\left(E_{T}\right)$ distribution would show an unexpected shift. Moreover, one needs to ensure that all units of the individual detector components are read out in the events of our interest. In order to study the performances of individual detector components, monitoring tools have been developed and the characteristic distributions for jets, electrons, muons, vertices, etc. are examined for each run ${ }^{\S}$. Each run is flagged correspondingly, before storing the data in a centralised database [73]. The present analysis uses a dataset which has gone through the following checks for each run.

- Missing $E_{T}$

\footnotetext{
$\S^{\S}$ The beam luminosity falls off over time; as a result $p \bar{p}$ collision rate decreases with time, as it is directly proportional to the beam luminosity. In the trigger system, different trigger conditions (electron, jet, muon, etc.) are allowed to select events at different rates relative to one another. This is called prescaling of triggers. In order to record the events (to tape) at a constant rate, the datasets are partitioned at suitable intervals of time, called runs. The prescaling factors for different triggers are altered at the beginning of a new run if the beam luminosity falls off considerably during the previous run. A run typically lasts for nearly four hours.
} 
- shift of $E_{T}: \sqrt{{\overline{E_{T}}}_{x}^{2}+{\overline{E_{T}}}_{y}^{2}}<4 \mathrm{GeV}$

- average rms of $\#_{T}$ distribution: $\sqrt{\sigma_{E / T x}^{2}+\sigma_{E / T y}^{2}}<16 \mathrm{GeV}$

- average of scalar $E_{T}$ i.e., the scalar sum of the transverse energy in an event: $\overline{\mathrm{S}_{\mathrm{T}}}>80 \mathrm{GeV}$

Fig. 4.2 shows distributions for above variables, used to monitor the data quality as a function of run number. In addition, it is ensured that the Calorimeter, Muon, SMT and CFT subsystems satisfy the following criteria during data-taking.

- no known hardware problem

- all units are read out

Apart from the hardware problems, an event can be lost at various stages of the readout process during its passage from the detector to the storage disk. These losses are potentially due to the software problems in online system, crashes in event reconstruction program, inconsistency in database entries, etc. Total integrated luminosity of a dataset is corrected to account for these losses [74] and the corrected luminosity is called "reconstructed" luminosity. For the present dataset, Tab. 4.1 summarizes the losses in luminosity due to the reasons described above. 

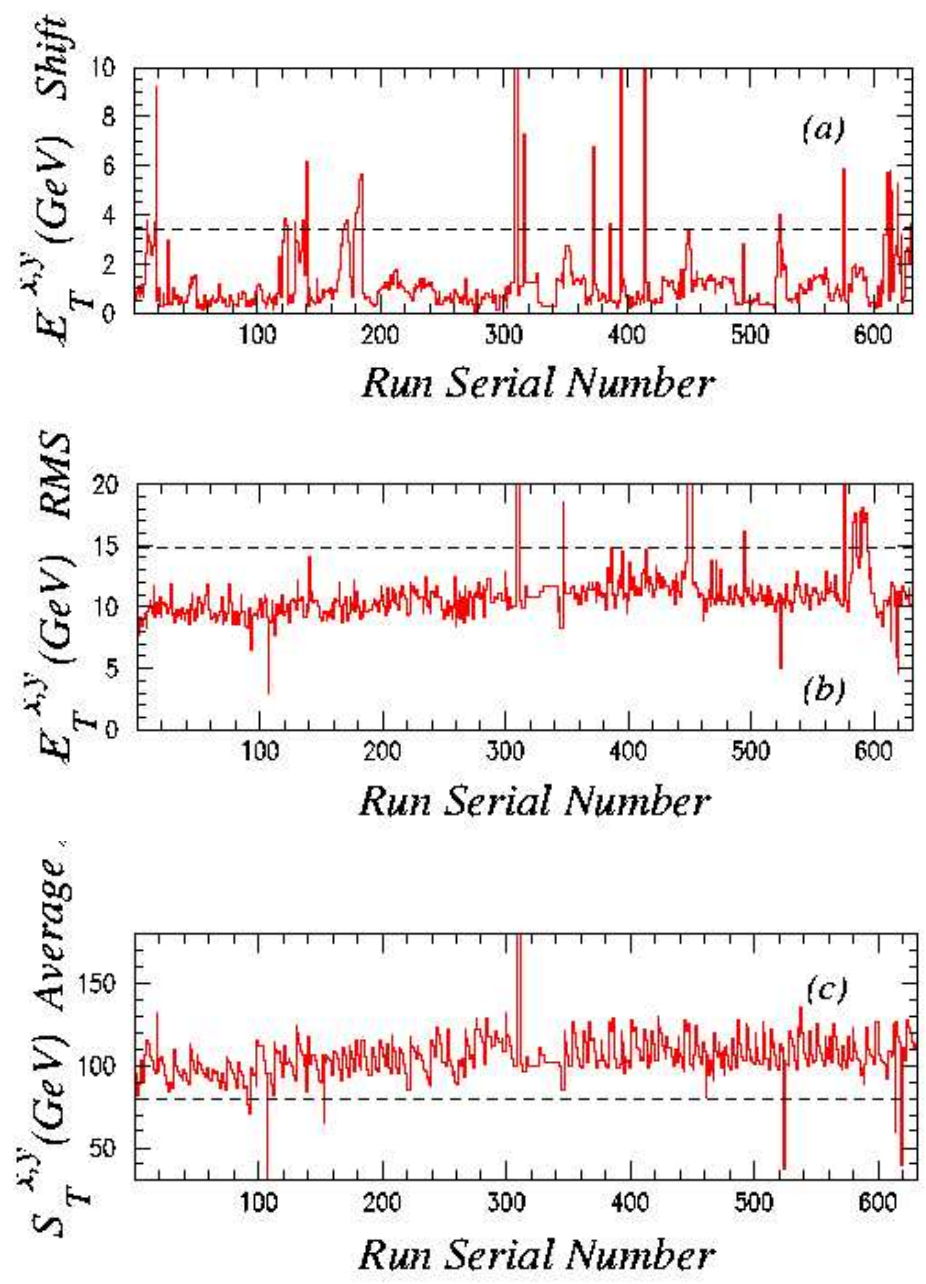

Figure 4.2: The shift (a) and RMS (b) of the missing transverse energy per run, as a function of the run serial number. The average (c) of the scalar transverse energy per run as a function of the serial run number. 


\begin{tabular}{l|c|c|c}
\hline Stage & Luminosity $\left(\mathrm{pb}^{-1}\right)$ & Relative Size (\%) & Absolute Size (\%) \\
\hline Delivered & 300.686 & 100 & 100 \\
Recorded & 263.955 & 87.784 & 87.784 \\
Data Quality & 230.617 & 87.369 & 76.697 \\
Reconstructed & 228.293 & 98.992 & 75.924 \\
\hline
\end{tabular}

Table 4.1: Summary of the integrated luminosity for the data sample.

\section{$4.2 \quad$ Trigger}

In order to select the events for this analysis, a particular trigger called $e \mu$ trigger has been implemented in the DØ trigger system (see Sec. 2.2.8). The trigger requires the following conditions

- One electromagnetic object at Level 1,

- One muon object at Level 1,

- One electromagnetic object at Level 3, matched to the Level 1 object.

Any data analysis requires accurate estimation of the trigger efficiency for the signal of interest. The trigger efficiencies for different physics processes are estimated from Monte Carlo events, as described below. The probability of a single object (electron/muon/jet) to satisfy the trigger requirement at different levels of triggering is obtained from data. The total probability of an event to satisfy the trigger condition is the product of the probabilities at 
each trigger level and can be written as,

$$
\mathrm{P}(\mathrm{L} 1 \mathrm{~L} 2 \mathrm{~L} 3)=\mathrm{P}(\mathrm{L} 1) \times \mathrm{P}(\mathrm{L} 2 \mid \mathrm{L} 1) \times \mathrm{P}(\mathrm{L} 3 \mid \mathrm{L} 1 \mathrm{~L} 2)
$$

where $\mathrm{P}(\mathrm{L} 1 \mid \mathrm{L} 2)$ and $\mathrm{P}(\mathrm{L} 3 \mid \mathrm{L} 1 \mathrm{~L} 2)$ represent the conditional probabilities for an event to satisfy a set of criteria provided it has already passed the requirements imposed at the previous trigger level(s). Under the assumption that the probabilities for different types of objects (viz. electron, muon) to pass the trigger conditions are uncorrelated, the probabilities at each level of triggering can be decomposed into

$$
\mathrm{P}\left(\text { Object }_{1}, \text { Object }_{2}\right)=\mathrm{P}\left(\text { Object }_{1}\right) \times \mathrm{P}\left(\text { Object }_{2}\right) .
$$

Furthermore, the probability that out of $\mathrm{N}$ similar objects in an event, at least one will satisfy the trigger condition is given by

$$
\mathrm{P}=1-\prod_{\mathrm{i}=1}^{\mathrm{N}}\left(1-\mathrm{P}_{\mathrm{i}}\right)
$$

where $P_{i}$ represents the single object probability obtained from data. It can be shown that the probability of at least two objects to satisfy a particular 


\begin{tabular}{|c|c|c|c|c|}
\hline Version & Trigger Name & Level 1 & Level 2 & Level 3 \\
\hline v12 & MATX_EM6_L12 & mu1ptxatxx_CEM $(1,6)$ & - & ELE_NLV $(1,12)$ \\
\hline v11 & MU_A_EM10 & mu1ptxatxx_CEM $(1,5)$ & - & ELE_LOOSE $(1,10)$ \\
\hline v10 & MU_A_EM10 & mu1ptxatxx_CEM $(1,5)$ & - & ELE_LOOSE $(1,10)$ \\
\hline v9 & MU_A_EM10 & mu1ptxatxx_CEM $(1,5)$ & - & ELE_LOOSE $(1,10)$ \\
\hline v8.2-v9 & MU_W_EM10 & mu1ptxatxx_CEM $(1,5)$ & - & ELE_LOOSE $(1,10)$ \\
\hline
\end{tabular}

Table 4.2: Triggers used in the electron-muon analysis; note there is no requirement at Level 2 of Trigger system.

trigger condition, out of $\mathrm{N}$ objects in an event is,

$$
P=1-\prod_{i=1}^{N}\left(1-P_{i}\right)-\sum_{i=1}^{N} P_{i} \prod_{j=1, j \neq i}^{N}\left(1-P_{j}\right)
$$

Since the beginning of Run II, D $\varnothing$ has been updating the trigger conditions for improved performance of the trigger system. Each major change is given a different version number. The current analysis uses datasets which are selected with v8.2-v12 (as shown in Tab. 4.2) versions of $e \mu$ trigger. The probability for an event to pass the $e \mu$ trigger is

$$
\mathrm{P}_{\mathrm{e} \mu}=\mathrm{P}_{\mathrm{e}} \cdot \mathrm{P}_{\mu}
$$

$P_{e}\left(P_{\mu}\right)$ represents the probability for one electron (muon) in an event to satisfy electron (muon) conditions at Level 1, Level 2 and Level 3. These probabilities can be further subdivided into individual trigger requirements 
(it should be noted that no Level 2 condition is required).

$$
\mathrm{P}_{\mathrm{e}}=\mathrm{P}(\mathrm{e} \mid \mathrm{L} 1) \cdot \mathrm{P}(\mathrm{e} \mid \mathrm{L} 3) \quad \mathrm{P}_{\mu}=\mathrm{P}(\mu \mid \mathrm{L} 1)
$$

Furthermore, to account for the fact that the jets can also fire the EM trigger at Level 1, P(e|L1) can be modified using 4.5.

$$
\mathrm{P}(\mathrm{e} \mid \mathrm{L} 1)=\mathrm{P}(\mathrm{e} \mid \mathrm{L} 1)+\mathrm{P}(\text { jet } \mid \mathrm{L} 1)-\mathrm{P}(\mathrm{e} \mid \mathrm{L} 1) \cdot \mathrm{P}(\mathrm{jet} \mid \mathrm{L} 1)
$$

It has been checked that $\mathrm{P}(\mathrm{e} \mid \mathrm{L} 3)$ has negligible contribution from the jet probability at Level 3. Each of these probabilities (viz. P(e|L1), P(jet|L1), $\mathrm{P}(\mathrm{e} \mid \mathrm{L} 3)$ and $\mathrm{P}(\mu \mid \mathrm{L} 1))$ has been derived from data and are described below.

\subsubsection{Single Object Trigger Efficiency}

The probability of a single object to satisfy a particular trigger requirement is measured using the following general procedure. At first, a sample of events, unbiased for the trigger under study, is selected. The offline reconstructed objects are then identified from all events in the sample. The efficiency is obtained by calculating the fraction of these offline reconstructed objects which satisfy the trigger condition under study. Single object efficiencies are 
in general parametrized as functions of the variables $\mathrm{p}_{\mathrm{T}}, \eta$ and $\phi$ of the offline reconstructed objects.

1. Electron: The efficiency for an offline electron to pass a specific trigger requirement is obtained by using a $Z^{0} \rightarrow e e$ sample with "tagand-probe" method. Events triggered by one of the single electron triggers are further selected by requiring the presence of two offline electrons. The invariant mass of the two offline electrons is required to be within a small window around $Z^{0}$ mass. One of the electrons is randomly chosen ("tag") and is required to satisfy a Level 1 electron trigger. The second electron ("probe") is then used to calculate efficiencies, separately for Level 1 and Level 3 trigger conditions. The single object electron trigger efficiency is found to be constant in $\eta$ and $\phi$; therefore it is parametrized as a function of the offline reconstructed electron, $p_{T}$ as.

$$
f\left(p_{T}\right)=0.5 \cdot \mathrm{A}_{2} \cdot\left(1+\operatorname{Erf}\left(\frac{\mathrm{p}_{\mathrm{T}}-\mathrm{A}_{0}}{\sqrt{2} \cdot \mathrm{A}_{1}}\right)\right)
$$

where $\operatorname{Erf}(\mathrm{x})=\frac{2}{\sqrt{\pi}} \cdot \int_{0}^{\mathrm{x}} \mathrm{e}^{-\mathrm{t}^{2}} \mathrm{dt}$, the standard error function. 
(a) Level 1: As mentioned earlier, $e \mu$ trigger requires a Level 1 trigger $\operatorname{CEM}(1,5)$ or $\operatorname{CEM}(1,6)$ depending on the trigger version. The terms $\operatorname{CEM}(1,5)$ and $\operatorname{CEM}(1,6)$ indicate that the event is required to fire at least one electromagnetic tower in the calorimeter with $E_{T}>5$ or $E_{T}>6 \mathrm{GeV}$, respectively. Since the current analysis deals with electrons of $p_{T} \geq 15 \mathrm{GeV}$, the profile of the turn-on curves in the region $p_{T} \sim 15 \mathrm{GeV}$ is of interest. It should noted that the profile of the turn-on curves have been checked in the region $p_{T}<15 \mathrm{GeV}$, with a sample of smaller statistics. Fig. 4.3 demonstrates that the trigger efficiency in the region $p_{T} \geq 15$ $\mathrm{GeV}$, is a constant.

(b) Level 3: The single object trigger efficiency for Level 3 electromagnetic objects are obtained similarly. Two triggers viz., ELE_LOOSE $(1,10)$ and ELE_NLV $(1,12)$ for trigger versions v8-v11 and v12 respectively are used at Level 3. The trigger ELELOOOSE $(1,10)$ (ELE_NLV $(1,12))$ requires one electro-magnetic shower in the calorimeter with $E_{T}>10(12) \mathrm{GeV}$. The trigger efficiency for an electron to satisfy the Level 3 requirement with energy threshold below $15 \mathrm{GeV}$ is described in Ref. [75]. By varying the 


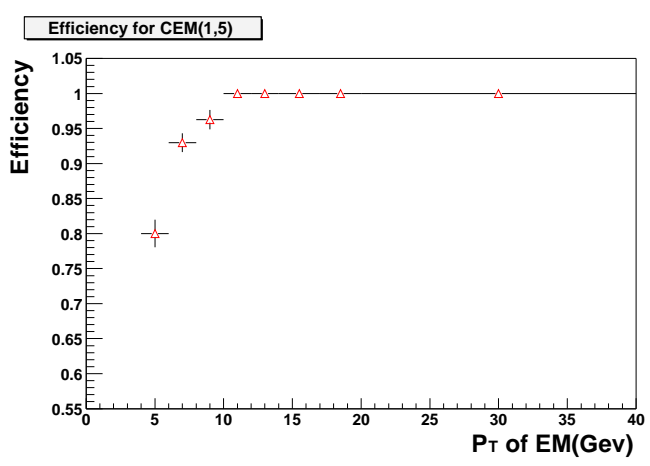

(a)

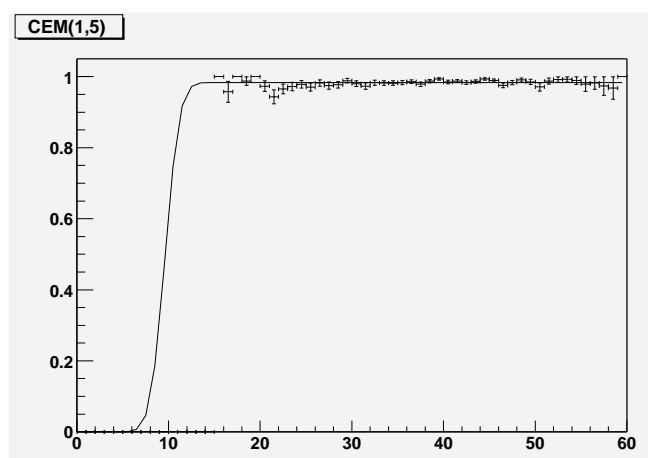

(b)

Figure 4.3: Level 1 electron trigger efficiencies as a function of $p_{T}$ of the electron from two different samples. (a) The sample with smaller statistics, shows that the Level 1 electron trigger becomes $100 \%$ efficient around $p_{T} \sim 10$ $\mathrm{GeV}$; (b) a sample with large statistics having offline electrons with $p_{T} \geq 15$ $\mathrm{GeV}$, shows that Level 1 electron trigger has 100\% efficiency for electrons with $p_{T} \sim 15 \mathrm{GeV}$.

energy thresholds, (say 20, 25, $30 \mathrm{GeV}$ ), corresponding turn-on parameters of Eqn. 4.9 are obtained; then by extrapolation, the parameters for a trigger with lower threshold is obtained.

The turn-on parameters viz., $\mathrm{A}_{0}, \mathrm{~A}_{1}$, and $\mathrm{A}_{2}$ of Eqn. 4.9 for Level 1 and Level 3 electromagnetic objects, are tabulated in Tab. 4.3.

2. Muon : The $e \mu$ trigger requires a Level 1 muon trigger viz., mu1ptxatxx, which is the condition for one reconstructed muon with $p_{T}>2 \mathrm{GeV}$. A sample of $Z^{0} \rightarrow \mu \mu$ events is used to obtain the muon trigger efficiencies. The "tag-and-probe" method is used to estimate the fraction 


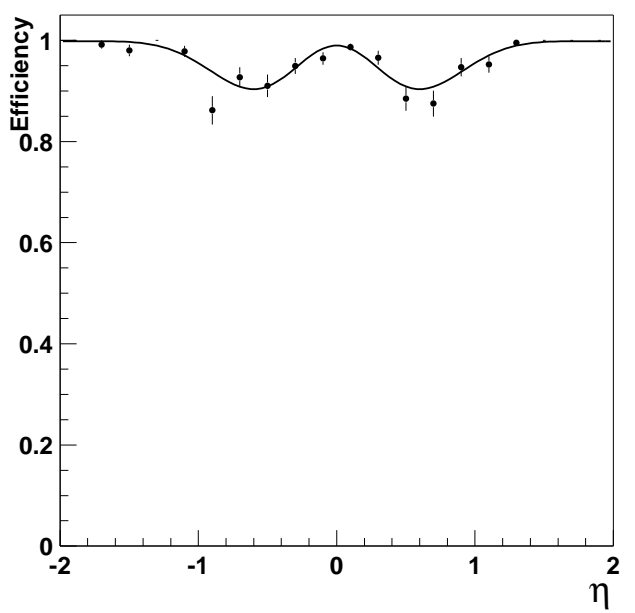

Figure 4.4: Level 1 muon trigger efficiencies as a function $\eta$ for mu1ptxatxx.

of offline muons that pass the muon trigger requirement at Level 1 . It has been found that the trigger efficiency is constant as a function of $p_{T}$, while it varies as a function of muon $\eta$ as,

$$
f(\eta)=\mathrm{A}_{3}+\mathrm{A}_{0} \cdot \exp \left(-\mathrm{A}_{1} \cdot\left(\eta^{2}-\mathrm{A}_{2}^{2}\right)\right) \cdot \sin \left(\eta^{2}-\mathrm{A}_{2}^{2}\right)
$$

Fig. 4.4 shows the variation of Level 1 muon trigger efficiency as a function of muon $\eta$. The parameters in Eqn. 4.10 are tabulated in Tab. 4.3. 
3. Jet : This analysis does not have any trigger requirement based on jets. However, as mentioned earlier in this section, the jets can fire the electron triggers at Level 1. This probability has been estimated using a sample which is selected by a muon trigger. To parametrize the turn-on curves for two different electron triggers, $\operatorname{CEM}(1,5)$ and $\operatorname{CEM}(1,6)$, the following equations are utilized,

$$
\begin{gathered}
f\left(p_{T}\right)=\left(\mathrm{A}_{2}+\mathrm{A}_{3} \cdot \mathrm{p}_{\mathrm{T}}\right) \cdot\left(1+\operatorname{Erf}\left(\frac{\mathrm{p}_{\mathrm{T}}-\mathrm{A}_{0}}{\sqrt{\mathrm{p}_{\mathrm{T}}} \cdot \mathrm{A}_{1}+\mathrm{A}_{4}}\right)\right) \\
f\left(p_{T}\right)=\left(\mathrm{A}_{2}+\mathrm{A}_{3} \cdot \mathrm{p}_{\mathrm{T}}\right) \cdot\left(1+\operatorname{Erf}\left(\frac{\mathrm{p}_{\mathrm{T}}-\mathrm{A}_{0}+\mathrm{A}_{4} \cdot \mathrm{p}_{\mathrm{T}}}{\sqrt{\mathrm{p}_{\mathrm{T}}} \cdot \mathrm{A}_{1}}\right)\right)
\end{gathered}
$$

The corresponding turn-on curves are shown in Figure 4.5 and the parameters are tabulated in Table 4.3. Figure 4.6 shows the probabilities of a jet to satisfy the Level 3 ELE_LOOSE(1,10) and ELE_NLV(1,12) requirements as a function of $p_{T}$. The efficiency is significantly low and thus neglected.

\subsubsection{Total Trigger Efficiency}

Since the event contributions from signal and many background processes in this analysis are based on Monte Carlo event simulation, it is highly desirable 


\section{$\operatorname{CEM}(1,5)$}
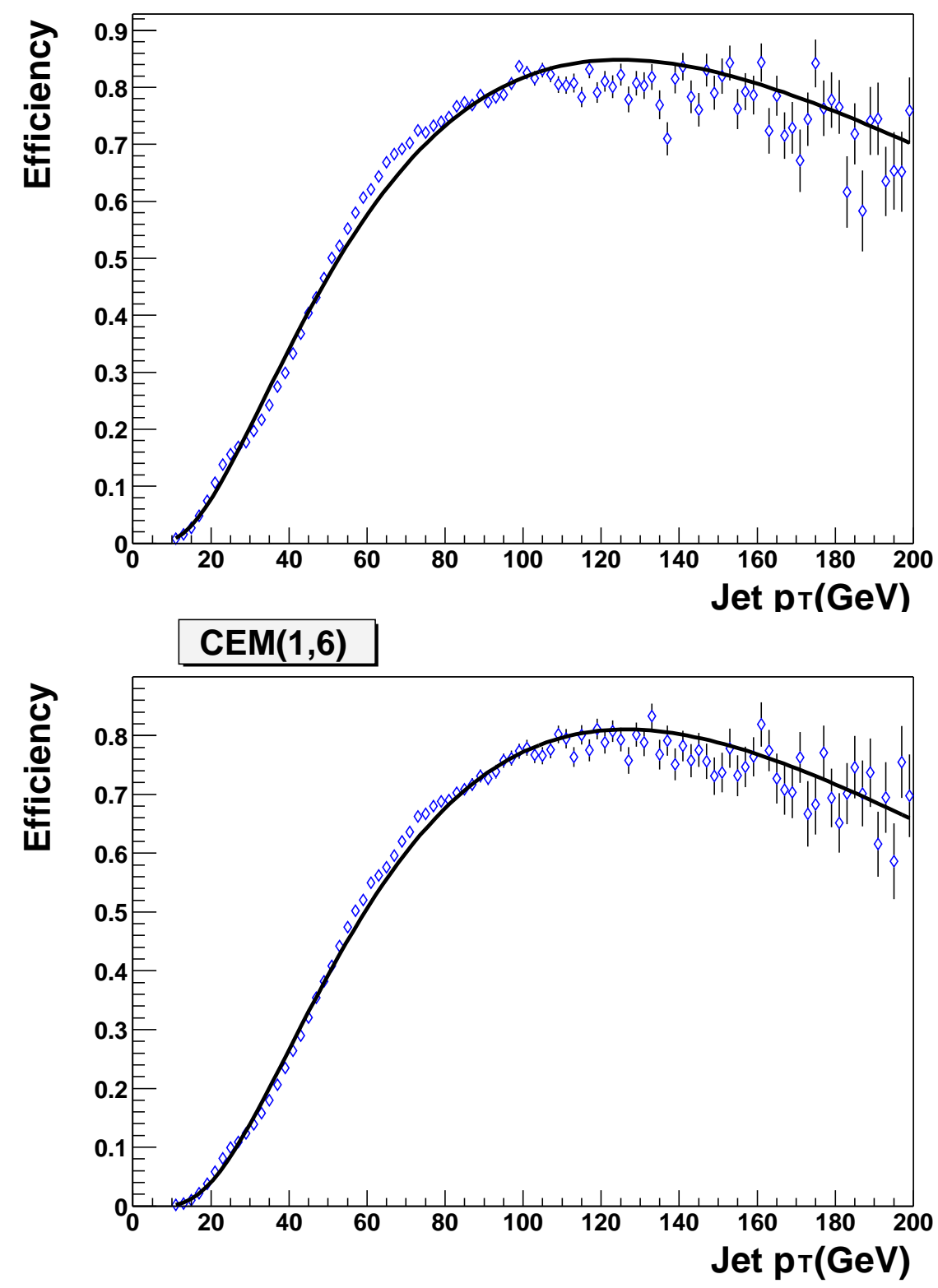

Figure 4.5: Probabilities for a jet to fire Level 1 electron trigger as a function of $p_{T}$. 

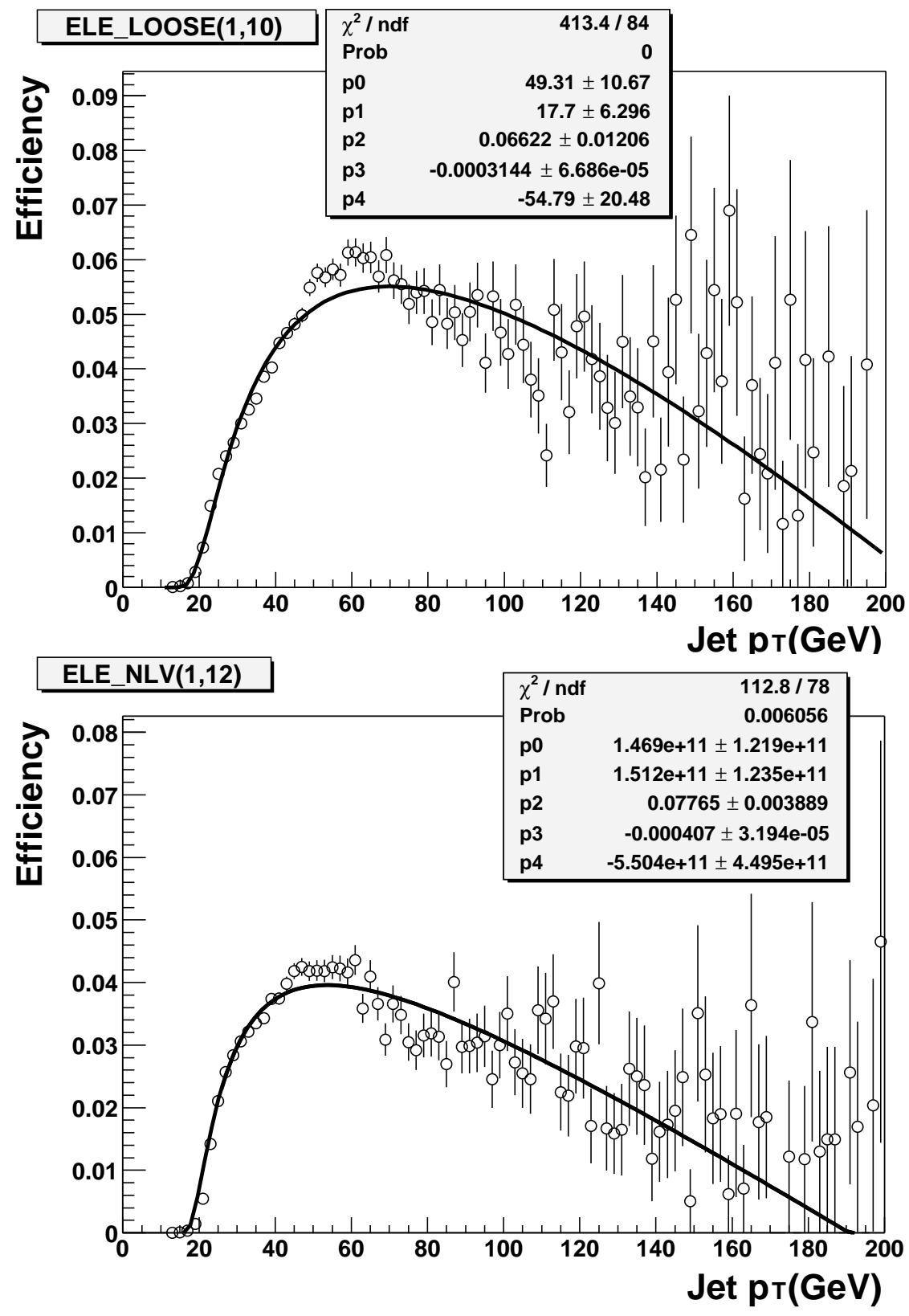

Figure 4.6: Probabilities for a jet to fire Level 3 electron trigger as a function of $p_{T}$. 


\begin{tabular}{|c|c|c|c|c|c|c|c|}
\hline \multirow[t]{2}{*}{ Object } & \multirow[t]{2}{*}{ Trigger Name } & \multirow[t]{2}{*}{ Equation } & \multicolumn{5}{|c|}{ Parameters } \\
\hline & & & $A_{0}$ & $\overline{A_{1}}$ & $A_{2}$ & $A_{3}$ & $A_{4}$ \\
\hline \multirow[t]{6}{*}{ Electron } & 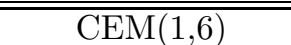 & "4.9 & $=$ & $=$ & 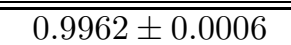 & $\overline{\mathrm{N} / \mathrm{A}}$ & $\overline{\mathrm{N} / \mathrm{A}}$ \\
\hline & $\operatorname{CEM}(1,5)$ & 4.9 & $=$ & $=$ & $0.983 \pm 0.001$ & $\mathrm{~N} / \mathrm{A}$ & $\mathrm{N} / \mathrm{A}$ \\
\hline & ELE_NLV $(1,12)$ & 4.9 & $13.6 \pm 0.5(\mathrm{CC})$ & $1.57 \pm 0.07(\mathrm{CC})$ & $0.974 \pm 0.002(\mathrm{CC})$ & $\mathrm{N} / \mathrm{A}$ & $\mathrm{N} / \mathrm{A}$ \\
\hline & & & $13.65 \pm 0.08(\mathrm{EC})$ & $1.4 \pm 0.1(\mathrm{EC})$ & $0.998 \pm 0.001(\mathrm{EC})$ & $\mathrm{N} / \mathrm{A}$ & $\mathrm{N} / \mathrm{A}$ \\
\hline & ELE_LOOSE $(1,10)$ & 4.9 & $13.8 \pm 0.7(\mathrm{CC})$ & $2.7 \pm 0.1(\mathrm{CC})$ & $0.977 \pm 0.004(\mathrm{CC})$ & $\mathrm{N} / \mathrm{A}$ & $\mathrm{N} / \mathrm{A}$ \\
\hline & & & $13 \pm 1(\mathrm{EC})$ & $3.3 \pm 0.2(\mathrm{EC})$ & $0.991 \pm 0.005(\mathrm{EC})$ & $\mathrm{N} / \mathrm{A}$ & $\mathrm{N} / \mathrm{A}$ \\
\hline Muon & mu1ptxatxx & 4.10 & $-0.8 \pm 0.2$ & $2.8 \pm 0.5$ & $0.1 \pm 0.1$ & $0.99 \pm 0.01$ & $\mathrm{~N} / \mathrm{A}$ \\
\hline \multirow[t]{2}{*}{ Jets } & $\operatorname{CEM}(1,6)$ & 4.12 & $66.8 \pm 0.5$ & $8.5 \pm 0.07$ & $11 \pm 1$ & $0.56 \pm 0.02$ & $-1.85 \pm 0.01$ \\
\hline & $\operatorname{CEM}(1,5)$ & 4.11 & $68.6 \pm 0.4$ & $10.49 \pm 0.009$ & $3.9 \pm 0.5$ & $0.32 \pm 0.006$ & $-2.001 \pm 0.006$ \\
\hline
\end{tabular}

Table 4.3: Parameters of the Trigger Turn-on curves used in the electron-muon analysis. 


\begin{tabular}{|c|c|}
\hline Monte Carlo Process & Trigger Efficiency (\%) \\
\hline \hline$t \bar{t} \rightarrow e \mu$ & $93.77 \pm 0.41$ \\
$W^{+} W^{-} \rightarrow e \mu$ & $92.52 \pm 0.66$ \\
$Z^{0} / \gamma^{*} \rightarrow \tau \tau$ & $91.24 \pm 0.92$ \\
$W^{ \pm} Z^{0} \rightarrow e \mu$ & $93.74 \pm 0.59$ \\
$Z^{0} / \gamma^{*} \rightarrow \mu \mu$ & $90.92 \pm 5.02$ \\
$W^{ \pm} \gamma \rightarrow \mu \nu_{\mu} \gamma$ & $90.38 \pm 0.76$ \\
$Z^{0} \gamma \rightarrow \mu \mu \gamma$ & $92.15 \pm 0.78$ \\
\hline
\end{tabular}

Table 4.4: Trigger efficiencies for different Monte Carlo processes. Errors are statistical.

that the trigger efficiencies for each of these individual processes are estimated accurately. Applying the procedure described above, the efficiencies at which the $e \mu$ trigger would select the events originating from different processes have been estimated using the objects from the corresponding Monte Carlo samples and the efficiencies are provided in Tab. 4.4 .

\subsection{Monte Carlo}

Monte Carlo event simulation is an integral part of any analysis for checking the consistency of experimental results. In High Energy Physics, the Monte Carlo event simulation consists of two parts. First part involves the hardscattered physics process and the subsequent "fragmentation and decay" of the partons. This is based on our theoretical understanding of a particu- 
lar physics process. Second part is detector specific as it requires detailed simulation of detector responses, when the particles produced in the physics process pass through it. The output is produced in the same format as that of real experimental data. The simulated events are passed through the reconstruction program for event reconstruction. In DØ Alpgen $1.2[76]$ or Pythia 6.2 [77] are used to simulate the hard-scattered processes and the fragmentation and decay are carried out using Pythia 6.2. The set of parton distribution functions (PDF) used for modelling the proton and anti-proton is CTEQ6.1M in Alpgen and CTEQ5L in Pythia. All physics processes described below are generated at $\sqrt{s}=1.96 \mathrm{TeV}$.

\subsubsection{Event Production}

\section{$t \bar{t}$ Signal Simulation}

Alpgen 1.2 has been utilized to generate a set of $t \bar{t} \rightarrow b W^{+} \bar{b} W^{-} \rightarrow l l(l=$ $e, \mu, \tau)$ events, with Pythia performing the hadronisation. At the parton level, the leptons are required to be within $|\eta|<10$, the jets are required have $p_{T}>8 \mathrm{GeV}$ and $|\eta|<3.5$ with a separation between two jets $\Delta R(j, j)>0.4$ in $\eta-\phi$ space. The branching fractions and lifetimes for various b-quark states: $\mathrm{B}^{0}, \mathrm{~B}^{+}, \mathrm{B}_{\mathrm{s}}^{0}, \mathrm{~B}_{\mathrm{c}}^{0}$ and $\Lambda_{\mathrm{b}}$ are obtained using EvtGen [78]. The cross 
section calculation for $t \bar{t}$ process is performed at a scale, $Q^{2}=m_{\mathrm{top}}^{2}+<p^{2}{ }_{T}>$ with $\mathrm{m}_{\text {top }}=175 \mathrm{GeV} / \mathrm{c}^{2}$. The Monte Carlo sample contains all dilepton final states including $\tau^{\prime}$ 's. Inclusive decays of $\tau$ leptons are obtained using TAUOLA [79].

\section{Background Simulation}

Various background processes which could contaminate the $t \bar{t} \rightarrow e \mu$ signal are considered in the present analysis. Many of these background processes become negligible after appropriate selection criteria, but they are studied for the purpose of consistency in background estimation. Two types of event generators (Alpgen and Pythia) are utilized for the simulation of the major background processes viz., $Z^{0} / \gamma^{*} \rightarrow \tau \bar{\tau} \rightarrow e \mu \nu_{e} \nu_{\mu} \nu_{\tau} \overline{\nu_{\tau}}$ and $W^{+} W^{-} \rightarrow e \mu$ decays, which survive after applying all selection criteria. The remaining background events are simulated using Pythia 6.2. The fragmentation and decay are always carried out with Pythia 6.2. The background channels for this analysis are classified into the following categories.

\section{Physics Backgrounds}

Decay processes with a real electron and a real muon in the final state, are called physics backgrounds. 
1. $Z^{0} / \gamma^{*} \rightarrow \tau \bar{\tau} \rightarrow e \mu \nu_{e} \nu_{\mu} \nu_{\tau} \overline{\nu_{\tau}}$ is the dominating background for $t \bar{t} \rightarrow e \mu$ analysis at the production level. A Pythia sample having large statistics is generated with $M_{\tau \bar{\tau}}>30 \mathrm{GeV}$, where the $\tau$ 's decay to electron or muon according to proper branching ratios. Events are further selected using a parton level cut of $p_{T}^{\text {e or } \mu}>8 \mathrm{GeV}$. The crosssection for this sample is calculated using the cross section for $Z^{0} \rightarrow$ $\mu^{+} \mu^{-}$measured by $\mathrm{D} \varnothing[80]$, corrected for the parton level cuts and is found to be $12.61 \pm 0.61 \mathrm{pb}$.

As Pythia does not generate the jets at the parton level, another sample of $Z^{0} / \gamma^{*} j j \rightarrow \tau \bar{\tau} j j$ has been studied separately to predict the contribution of this background process at the final stage of the present analysis. This sample has been generated with the condition $60<M_{\tau \bar{\tau}}<130$ $\mathrm{GeV}$, using the Alpgen generator with leading order matrix elements for the process. At the parton level the leptons are required to have $|\eta|<10$. The jets are generated with $p_{T}>8 \mathrm{GeV}$ and $|\eta|<3.5$ while two jets are required to have $\Delta R(j, j)>0.4$ (in $\eta-\phi$ space) at the parton level. The factorisation scale for this process is set to $Q^{2}=M_{Z}+\sum p_{T}^{2}$ and the $\tau$ 's are forced to decay into leptons. Cross section for this sample is taken as $1.90 \pm 0.05 \mathrm{pb}$, which is given by 
Alpgen. It has been checked that the contributions from lower and higher mass windows i.e. $M_{\tau \bar{\tau}}<60$ and $M_{\tau \bar{\tau}}>130$ are negligible and thus have not been considered.

2. $\boldsymbol{W}^{+} \boldsymbol{W}^{-} \rightarrow \boldsymbol{e} \boldsymbol{\mu}$ events are very similar to $t \bar{t} \rightarrow e \mu$ events in terms of lepton and neutrino $p_{T}$ 's, apart from the fact that the jets in $t \bar{t}$ are much more energetic. Pythia and Alpgen generators are used to produce $W^{+} W^{-} \rightarrow l^{+} l^{-}$and $\left(W^{+} W^{-} j j \rightarrow l^{+} l^{-}\right) j j$ samples respectively $(l=e, \mu, \tau)$. Fragmentation and decay are performed using Pythia and inclusive decays of $\tau$-leptons are set using Tauola. The $W^{+} W^{-}$sample is normalised to the NLO cross section, which is $35 \%$ higher than the LO calculations [81]. Unfortunately, NLO calculation of $\left(W^{+} W^{-} \rightarrow l^{+} l^{-}\right) j j$ production cross section is not available. To be consistent, the LO cross section reported by Alpgen for $\left(W^{+} W^{-} \rightarrow l^{+} l^{-}\right) j j$, is also scaled up by $35 \%$ and a $35 \%$ systematic uncertainty is assigned to the normalisation of this background. Cross sections of $1.38 \pm 0.03 \mathrm{pb}$ and $0.29 \pm 0.10 \mathrm{pb}$ for $W^{+} W^{-} \rightarrow l^{+} l^{-}$and $\left(W^{+} W^{-} j j \rightarrow l^{+} l^{-}\right) j j$ samples respectively, are used in this analysis.

3. $\boldsymbol{W}^{ \pm} \boldsymbol{Z}^{0} \rightarrow \boldsymbol{\mu} \boldsymbol{\nu}_{\boldsymbol{\mu}} \boldsymbol{e}^{+} \boldsymbol{e}^{-} / \boldsymbol{e} \boldsymbol{\nu}_{\boldsymbol{e}} \boldsymbol{\mu}^{+} \boldsymbol{\mu}^{-}$decays contribute a small amount at the production level and have almost negligible impact $(0.002 \pm 0.000)$ 
at the final stage of the current analysis. A Pythia sample has been generated where both $W^{ \pm}$and $Z^{0}$ decay leptonically i.e. $W^{ \pm} Z^{0} \rightarrow$ $l^{ \pm} \nu_{l} l^{+} l^{-},(l=e$ or $\mu)$. At the parton level, only the events containing at least one electron and one muon with $p_{T}^{e, \mu}>15 \mathrm{GeV}$ are selected. The NLO cross section [81] multiplied with the branching ratios provides a cross section of $0.014 \pm 0.005 \mathrm{pb}$ for this process.

\section{Photon Backgrounds}

Photon backgrounds contribute to the $e \mu$ channel where the events contain a muon and a photon (misidentified as an electron). Two types of photons can mimic an electron candidate.

1. Muon Bremsstrahlung: A muon can radiate a bremsstrahlung photon in the detector and the photon energy cluster in the calorimeter can get associated to the muon track in the tracking system. Here the direction of the misidentified electron in the transverse plane would be very close to the muon direction and applying a cut on $\Delta R(e, \mu)=$ $\sqrt{\Delta \eta^{2}+\Delta \phi^{2}}$ reduces this background by a significant amount. Following two physics processes can result in the above scenario. 
(a) $\boldsymbol{W} \rightarrow \boldsymbol{\mu} \boldsymbol{\nu}_{\boldsymbol{\mu}}$ gets completely rejected by the $\Delta R(e, \mu)$ cut. To check the consistency with experimental data, Pythia events are generated with CTEQ5L PDF to calculate the event yields at a selection level with $\geq 0$ jets. A cross section of $3226 \pm 100 \pm 128$ pb [82], measured by $\mathrm{D} \varnothing$ is used for this sample.

(b) $Z^{0} / \gamma^{*} \rightarrow \boldsymbol{\mu}^{+} \boldsymbol{\mu}^{-}$events survive the $\Delta R(e, \mu)$ cut when the muon emitting the bremsstrahlung photon, does not get reconstructed in the muon chambers. A large Pythia sample has been generated with with $60<M_{\mu^{+} \mu^{-}}<130 \mathrm{GeV}$ to study this background. The cross section is normalized to the $D \varnothing$ measured value of $261.8 \pm 5.0$ (stat) \pm 8.9 (syst) pb [80]. The referred cross section is a measurement only for $Z^{0}$ production with $M_{\mu^{+} \mu^{-}}>30$ $\mathrm{GeV}$, which does not include the contributions from $\gamma^{*}$ exchange and $\gamma^{*}-Z^{0}$ interference. Thus in order to include the contribution from Drell-Yan process, the cross section is corrected as,

$$
\begin{gathered}
\sigma\left(Z^{0} / \gamma^{*} \rightarrow \mu^{+} \mu^{-} ; M_{\mu^{+} \mu^{-}}>30 \mathrm{GeV}\right) \\
=\frac{\sigma\left(Z^{0} \rightarrow \mu^{+} \mu^{-} ; M_{\mu^{+} \mu^{-}}>30 \mathrm{GeV}\right)}{\frac{\sigma\left(Z^{0} \rightarrow \mu^{+} \mu^{-} ; M_{\mu^{+} \mu^{-}}>30 \mathrm{GeV}\right)}{\sigma\left(Z^{0} / \gamma^{*} \rightarrow \mu^{+} \mu^{-} ; M_{\mu^{+} \mu^{-}}>30 \mathrm{GeV}\right)}} \\
=\frac{261.8 \pm 10.2 \mathrm{pb}}{0.786 \pm 0.013}=333.1 \pm 14.1 \mathrm{pb}
\end{gathered}
$$


where the factor $0.786 \pm 0.013$ is obtained using Pythia. The D $\varnothing$ measured cross section is further corrected for the generated mass range viz., $60<\mathrm{M}_{\mu^{+} \mu^{-}}<130 \mathrm{GeV}$ as,

$$
\begin{aligned}
& \frac{\sigma\left(Z^{0} / \gamma^{*} \rightarrow \mu^{+} \mu^{-} ; 60<\mathrm{M}_{\mu^{+} \mu^{-}}<130 \mathrm{GeV}\right)}{\sigma\left(Z^{0} / \gamma^{*} \rightarrow \mu^{+} \mu^{-} ; M_{\mu^{+} \mu^{-}}>30 \mathrm{GeV}\right)} \\
= & \frac{\sigma^{\text {Alpgen }}\left(Z^{0} / \gamma^{*} \rightarrow l l ; 60<\mathrm{M}_{l l}<130 \mathrm{GeV}\right)}{\sigma^{\operatorname{Alpgen}}\left(Z^{0} / \gamma^{*} \rightarrow l l ; M_{l l}>30 \mathrm{GeV}\right)}
\end{aligned}
$$

where $\sigma^{\text {Alpgen }}\left(Z^{0} / \gamma^{*} \rightarrow l l ; 60<\mathrm{M}_{l l}<130 \mathrm{GeV}\right)$ and $\sigma^{\text {Alpgen }}\left(Z^{0} / \gamma^{*} \rightarrow l l ; M_{l l}>30 \mathrm{GeV}\right)$ are the leading order cross sections reported by Alpgen for $Z^{0}+0$ jet events. Thus the cross section for the $Z^{0} / \gamma^{*} \rightarrow \mu^{+} \mu^{-}\left(60<\mathrm{M}_{\mu^{+} \mu^{-}}<130 \mathrm{GeV}\right) \mathrm{MC}$ sample is thus found to be $266.5 \pm 11.8 \mathrm{pb}$.

2. Associated photon production: In contrast to the bremsstrahlung photons, these photons are produced in the hard-scattered events and have very small contribution to the $t \bar{t} \rightarrow e \mu$ event topology after applying the final selection criteria. Here the photon either gets associated with a random track or gets converted into an $e^{+} e^{-}$pair in the tracking system and one of them $\left(e^{+}\right.$or $\left.e^{-}\right)$appears as an electron candidate. The associated production of $\boldsymbol{W}^{ \pm}\left(\rightarrow \boldsymbol{\mu} \boldsymbol{\nu}_{\boldsymbol{\mu}}\right)$ or $\boldsymbol{Z}^{0}\left(\rightarrow \boldsymbol{\mu}^{+} \boldsymbol{\mu}^{-}\right)$with a 
photon are the sources of these backgrounds. These events have been generated with Pythia 6.2. The absolute cross sections for these samples are calculated with the Baur Monte Carlo [83] package, which is a better model for this calculation. These processes are generated using CTEQ5L version of PDF. Parton levels cuts of $\Delta R(\mu, \gamma)>0.25$ (in $\eta-\phi$ space) and $E_{T}^{\gamma}>10 \mathrm{GeV}$ have been applied, to remove the collinear divergences in cross section calculation for small values of $\Delta R(\mu, \gamma)$ and $E_{T}^{\gamma}$. The cross sections are $21.2 \pm 3.3 \mathrm{pb}$ and $8.3 \pm 0.5 \mathrm{pb}$ for $W^{ \pm} \gamma$ and $Z^{0} \gamma$ processes respectively, as calculated by the Baur MC package. The $\Delta R(\mu, \gamma)$ cut is varied between 0.15 and 0.35 in order to calculate a systematic uncertainty on the $W \gamma$ and $Z^{0} \gamma$ cross sections.

\subsubsection{Event Simulation}

A detailed detector simulation is necessary to understand the acceptance, smearing and other systematic effects. The generated Monte Carlo events are passed through the $\mathrm{D} \varnothing$ detector simulation and event reconstruction chain. The detector responses due to the passage of particles, are modelled properly, by specifying the detector material and geometry inside a software package dØgstar, which is based on GEANT 3 [84]. It incorporates the 
present understanding of the $\mathrm{D} \varnothing$ detector and results from test beam data.

In order to incorporate the possibility of having more than one interaction in a $p \bar{p}$ bunch crossing, events from low $p_{T}$ scattering processes are added to the hard scattered event. Processes like low $p_{T}$ QCD interactions, single diffraction, double diffraction, etc., collectively called minimum bias events, are generated separately using Pythia 6.2. The number of interactions in a $p \bar{p}$ bunch crossing follows a Poisson distribution and is proportional to the beam luminosity [85]. The data for the present analysis corresponds to 0.8 minimum bias interactions on average. After overlaying 0.8 minimum bias events on a hard scattered event, the output of dØgstar is digitized and written in a format similar to that of raw experimental data. This step is known as $d \emptyset$ sim. Finally the output of $d \emptyset$ sim is passed through the event reconstruction program, dØreco (detailed in Chap. 3).

\subsubsection{Monte Carlo Smearing}

Since the detector responses are not perfectly modelled in dØgstar, resolutions of kinematic variables of electron, muon or jet in data differ from those in Monte Carlo. In order to make MC samples represent experimental data more closely, distributions of these variables are smeared in Monte Carlo us- 
ing certain prescription. The smearing parameters are derived by comparing energy distributions of electron/muon/jet in experimental data with those in Monte Carlo samples.

\section{Electron Smearing}

A comparison of the di-electron invariant mass distribution in $Z^{0}$ candidate events shows width of the mass distribution is broader in data than in MC. There is also a difference in the peak position of the mass distributions in data and MC. Therefore, appropriate scale factor and smearing are used to make the electron energy distribution in MC more data like. The energy resolution of electrons as measured by the calorimeter, can be parametrized as

$$
\frac{\sigma(E)}{E}=C \oplus \frac{S}{\sqrt{E}} \oplus \frac{N}{E},
$$

where, $C, S$, and $N$ represent the constant, sampling and noise terms, respectively. Therefore one may use the following parametrization to adjust 
the scale and width of electron's energy distribution in Monte Carlo:

$$
\begin{aligned}
E^{\prime}=E \times[\alpha & +\xi_{1}(=\operatorname{Gaus}(0, \sigma=\alpha c)) \\
& +\xi_{2}(=\operatorname{Gaus}(0, \sigma=s \sqrt{\alpha / E})) \\
& \left.+\xi_{3}(=\operatorname{Gaus}(0, \sigma=n / E))\right]
\end{aligned}
$$

where, $\alpha$ is the scale factor, and $\xi_{1}, \xi_{2}$ and $\xi_{3}$ provide additional smearing to the width of the energy distribution. The variables $c, s$ and $n$ are the smearing coefficients in the constant, sampling and noise terms. It has been seen [86] that the scale factor and the smearing provided by $\sigma=\alpha c$ are sufficient to tune the electron energy in $\mathrm{MC}$ to that in data.

To study the electron energy scale and resolution, $Z^{0} \rightarrow e e$ data and Monte Carlo sample are used where the events have two isolated trackmatched electron clusters with $p_{T}>15 \mathrm{GeV}$. A comparison of the position and width of the $Z^{0} / \gamma^{*} \rightarrow e e$ peak in data and Monte Carlo can be seen in Fig. 4.7. We observe that the peak in the $Z^{0}$-mass distribution is shifted by about $0.002-0.040$ in $\mathrm{MC}$, and the width is narrower by about $0.33-0.46$ in $\mathrm{MC}$ relative to that in data for the different cases shown in the figure. These difference are corrected by scaling the electron energy in MC and by applying a smearing with first two terms of Eqn. 4.16. 

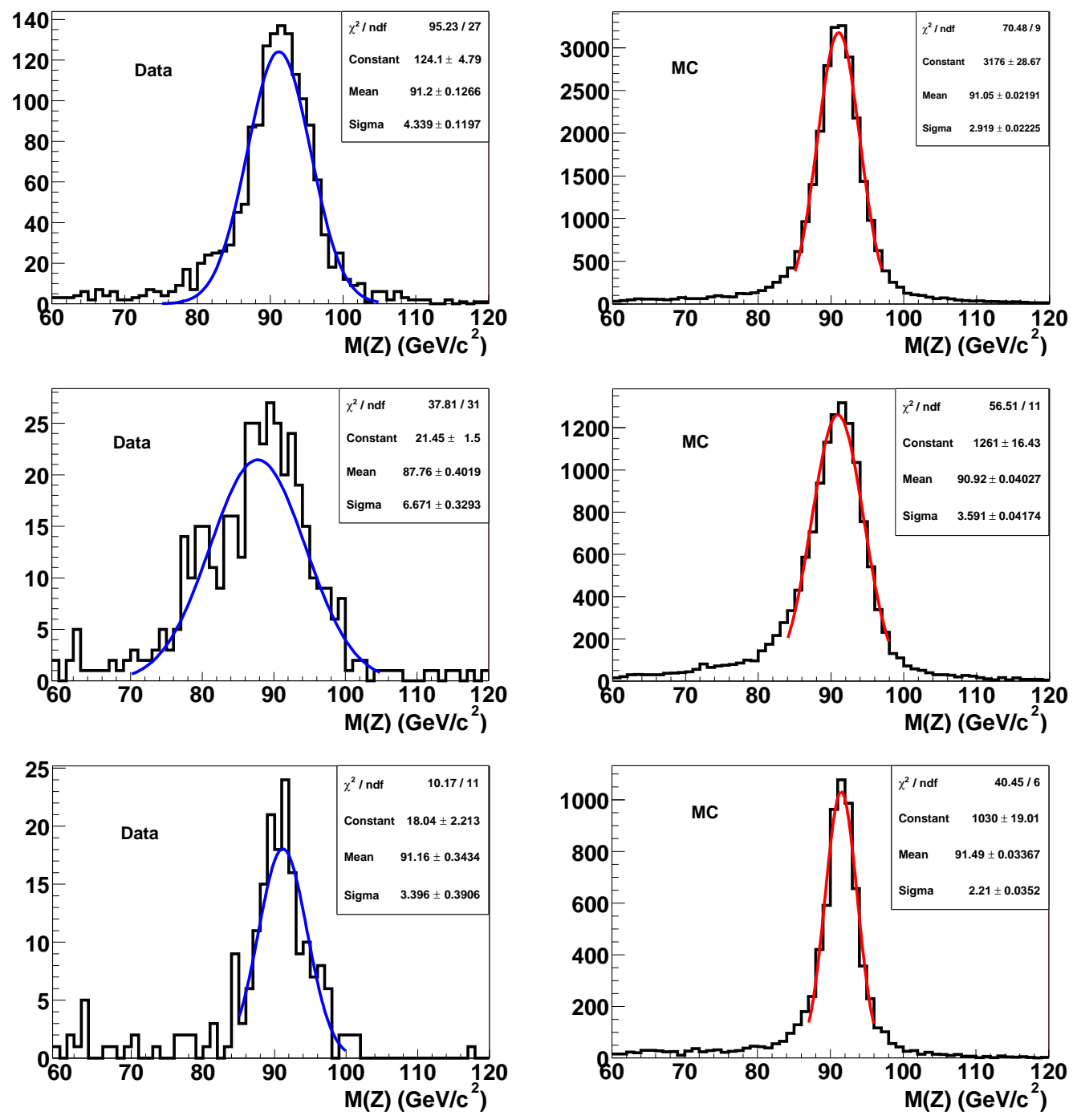

Figure 4.7: Distribution of di-electron invariant mass for data and MC $Z^{0} / \gamma^{*} \rightarrow e e$ events, with a Gaussian fit, for the case (i) with both electrons in the $\mathrm{CC}$ and in the fiducial regions of the detector (top), with (ii) both electrons in the $\mathrm{CC}$ and any one electron not in the fiducial region (middle), and (iii) with both electrons in the EC (bottom). 


\begin{tabular}{|c||c|c|c|}
\hline \hline Parameters & CCin & CCout & $E C$ \\
\hline$\alpha$ & 1.007 & 0.971 & 0.996 \\
$c$ & 0.042 & 0.083 & 0.031 \\
\hline \hline
\end{tabular}

Table 4.5: Values for scale factor $(\alpha)$ and smearing parameter (c) of Eqn. 4.16 for different types of electrons.

The scale factor, $\alpha$, and the width, $\sigma$, are obtained by varying $\alpha$ and $c$ and finding the best Kolmogorov-Smirnov (KS) match between the $Z^{0}$ mass distributions in data and MC. The scale factor and the smearing term are determined separately for the following categories of electrons in Monte Carlo:

- Electrons in the $\mathrm{CC}$ and in the fiducial ${ }^{\ddagger}$ regions of the detector $(\mathrm{CC} i n)$

- Electrons in the $\mathrm{CC}$ and not in the fiducial regions of the detector (CCout)

- Electrons in the EC $(E C)$

After the smearing the $Z^{0}$ mass peak is shown in Fig. 4.8, while the smearing parameters are tabulated in Tab. 4.5.

\footnotetext{
${ }^{\ddagger}$ The Central Calorimter is divided into 32 modules in $\phi$; the electrons passing through the transition regions between these modules (called " $\phi$ cracks"), are called non-fiducial electrons. The details of such regions can be found in Sec. 3.2.2.
} 


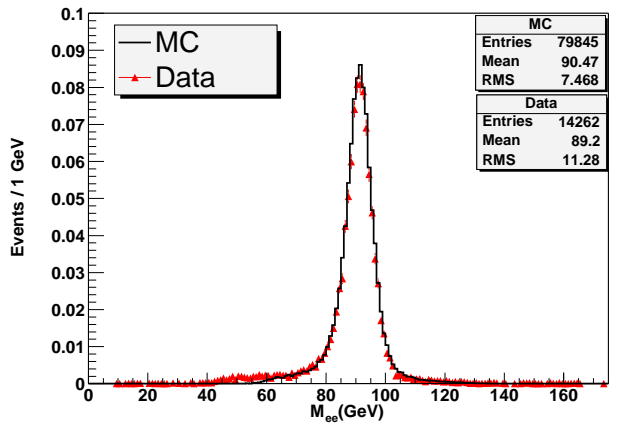

(a)

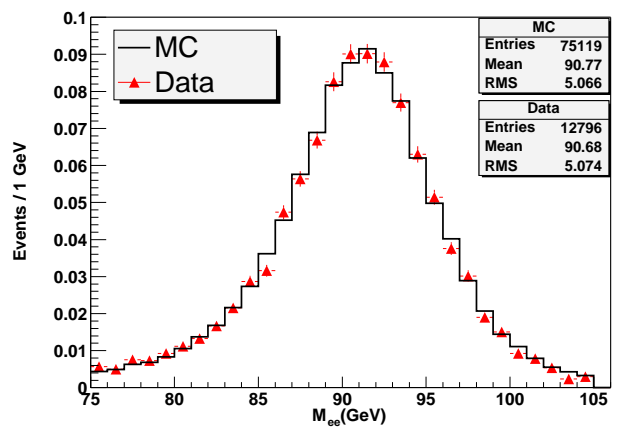

(b)

Figure 4.8: $M_{e e}$ distribution in ee events with zero or more jets. The smeared $Z^{0}$ Monte Carlo is compared with data containing two electrons. (a) shows the distribution in a range of $0-175 \mathrm{GeV}$ (b) shows the distribution in a range of $75-105 \mathrm{GeV}$.

\section{Muon Smearing}

Muon momentum scale and resolution in Monte Carlo are corrected by comparing the position and width of the $Z^{0} / \gamma^{*} \rightarrow \mu^{+} \mu^{-}$mass peak in the data to that in the Monte Carlo sample. The difference in the $Z^{0}$ peak position and width can be corrected in $\mathrm{MC}$, by scaling the muon $p_{T}$ in the Monte Carlo and applying an additional smearing,

$$
\frac{1}{p_{\mathrm{T}}^{\prime}}=\frac{1}{\alpha \cdot p_{\mathrm{T}}}+\xi
$$

where $\alpha$ is a scale factor and $\xi$ is a random Gaussian correction. Both $\alpha$ and the width of the Gaussian, $\sigma_{\xi}$, are obtained by requiring the best 

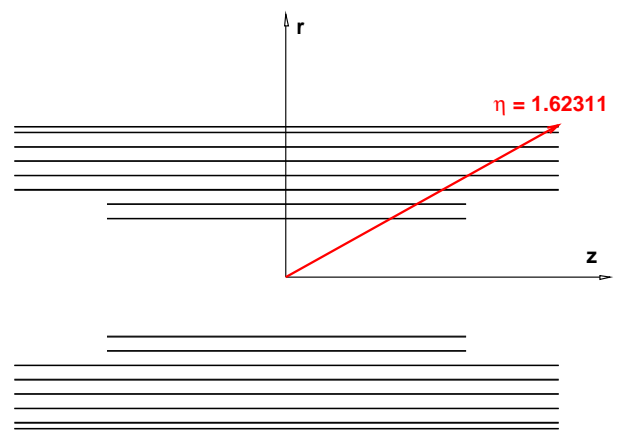

Figure 4.9: CFT coverage

Kolmogorov-Smirnov (KS) match between the $Z^{0}$ width in the data and Monte Carlo. Since the muon momentum resolution is determined by the tracking system, the comparison is made separately for two detector $\eta$ regions: the region with full CFT coverage (with 16 measurement points available, called the central region), $\left|\eta_{\text {det }}\right|<1.62$, and the forward region, $\left|\eta_{\text {det }}\right| \geq 1.62$ (Fig. 4.9). First, the procedure is applied only to the events with both muons in the central region. After the scale factor and the additional smearing correction have been determined for the central muons, the rest of the sample is treated in the following way. For the events with both muons in the forward region, the procedure is the same as above. For those events with one muon in the central and the other in the forward region, the 
central muon $p_{T}$ is corrected using the fixed parameters obtained from the central-central case, while the scale factor and amount of additional smearing for the forward muon $p_{T}$ are varied to find the best KS match probability. For the muons in the central region, the best match between the data and Monte Carlo is observed at,

$$
\begin{aligned}
\sigma_{\xi}^{\text {central }} & \simeq 0.0025 \mathrm{c} / \mathrm{GeV} \\
\alpha^{\text {central }} & \simeq 0.991
\end{aligned}
$$

and for the muons in the forward region the values are,

$$
\begin{aligned}
\sigma_{\xi}^{\text {forward }} & \simeq 0.0043 \mathrm{c} / \mathrm{GeV} \\
\alpha^{\text {forward }} & \simeq 0.996
\end{aligned}
$$

After smearing, the comparison between data and MC is shown in Fig. 4.10.

\section{Jet Smearing}

For high energy jets above $p_{T} \sim 50 \mathrm{GeV}$, the comparison between jet energy resolution in data and MC, is done using dijet event samples. The samples 


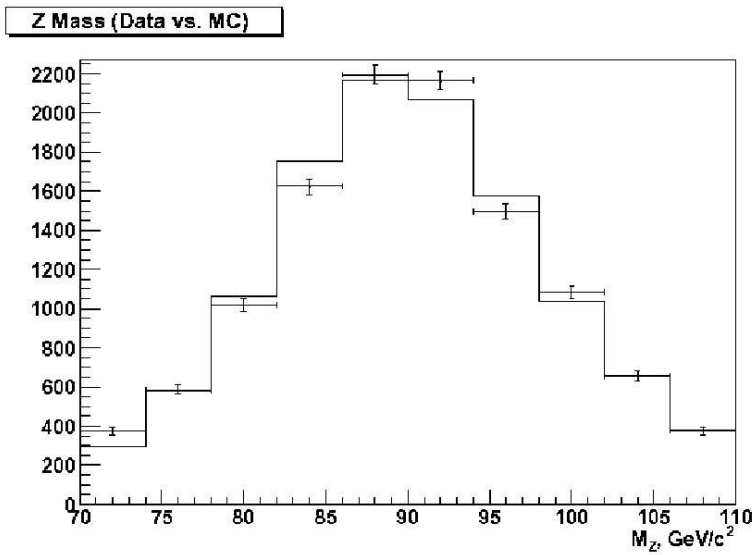

Figure 4.10: Comparison of the dimuon invariant mass in data (markers) and smeared Monte Carlo (solid histogram) in the $Z^{0}$ mass window.

are split in several bins of average $p_{T}$ of the dijet system, $<p_{T}>=\left(p_{T 1}+\right.$ $\left.p_{T 2}\right) / 2$, and for each $\left\langle p_{T}>\right.$ bin, distribution of the transverse momentum asymmetry variable, $\mathcal{A}$ :

$$
\mathcal{A}=\frac{\left|p_{T 1}-p_{T 2}\right|}{p_{T 1}+p_{T 2}}
$$

is studied. The width of the distribution of $\mathcal{A}, \sigma_{\mathcal{A}}$, obtained from a Gaussian fit with a mean value set to zero, gives jet $p_{T}$ resolution through the formula:

$$
\frac{\sigma_{p_{T}}}{p_{T}}=\sqrt{2} \sigma_{\mathcal{A}}
$$


For selecting the dijet event sample, the main requirements are that an event is triggered by a single jet trigger, the two jets pass standard jet quality criteria, and that they are back-to-back in the azimuthal plane. The single jet trigger with a $E_{T}$ threshold of $25 \mathrm{GeV}$ (JT_25TT_NG), is used for this purpose. This trigger becomes fully efficient for jet $p_{T}$ above $40 \mathrm{GeV}$, and therefore, the resolutions are measured only above $40 \mathrm{GeV}$.

In order to obtain the resolution for the jet $p_{T}$ range below $50 \mathrm{GeV}$, back-to-back $\gamma+$ jet events are used. These are triggered by single a EM trigger with no track requirement. The asymmetry variable for these events is defined as,

$$
A_{p j}=\frac{p_{T}^{j e t}-p_{T}^{\gamma}}{p_{T}^{\gamma}}
$$

Given that the energy resolution of $\gamma$ 's is much better than the energy resolution of hadronic jets, $\sigma_{p_{T}^{\gamma}}$ can be ignored compared to $\sigma_{p_{T}^{j e t}}$, and jet energy resolution can be expressed as:

$$
\frac{\sigma_{p_{T}^{j e t}}}{p_{T}^{j e t}}=\sigma_{A_{p j}} \times R_{p j}
$$

where the factor $R_{p j}=p_{T}^{\gamma} / p_{T}^{j e t}$ is used to correct imbalance between an average jet and photon $p_{T}$ 's in each $p_{T}$ bin. The results obtained from dijet 


\begin{tabular}{|l|c|c|c|c|}
\hline Category & $\left|\eta_{\text {det }}\right|$ range & $N$ & $S$ & $C$ \\
\hline Monte Carlo & $0.0<\left|\eta_{\text {det }}\right|<0.5$ & 4.26 & 0.658 & 0.0436 \\
& $0.5<\left|\eta_{\text {det }}\right|<1.0$ & 4.61 & 0.621 & 0.0578 \\
& $1.0<\left|\eta_{\text {det }}\right|<1.5$ & 3.08 & 0.816 & 0.0729 \\
& $1.5<\left|\eta_{\text {det }}\right|<2.0$ & 4.83 & 0. & 0.0735 \\
\hline Data & $0.0<\left|\eta_{\text {det }}\right|<0.5$ & 5.05 & 0.753 & 0.0893 \\
& $0.5<\left|\eta_{\text {det }}\right|<1.0$ & 0. & 1.20 & 0.0870 \\
& $1.0<\left|\eta_{\text {det }}\right|<1.5$ & 2.24 & 0.924 & 0.135 \\
& $1.5<\left|\eta_{\text {det }}\right|<2.0$ & 6.42 & 0. & 0.0974 \\
\hline
\end{tabular}

Table 4.6: Jet resolution constants for MC and data.

and $\gamma+$ jet samples are combined together and fitted using a common formula:

$$
\frac{\sigma_{p_{T}}}{p_{T}}=\sqrt{\frac{N^{2}}{p_{T}^{2}}+\frac{S^{2}}{p_{T}}+C^{2}} .
$$

The combined results are shown in Figs. 4.11, and 4.12, for the MC and data, respectively. The fit parameters are summarised in Tab. 4.6. The values of these parameters are used to the jet $p_{T}$ 's in $\mathrm{MC}$ events with a random Gaussian distribution of width

$$
\sigma=\sqrt{\sigma_{\mathrm{PT}_{\mathrm{T}}}^{\mathrm{data}^{2}}-\sigma_{\mathrm{PT}_{\mathrm{T}}}^{\mathrm{MC}}},
$$

where $\sigma_{\mathrm{PT}}^{\mathrm{MC}}, \sigma_{p_{T}}^{\text {data }}$ are the resolutions, estimated in MC and data events respectively. 

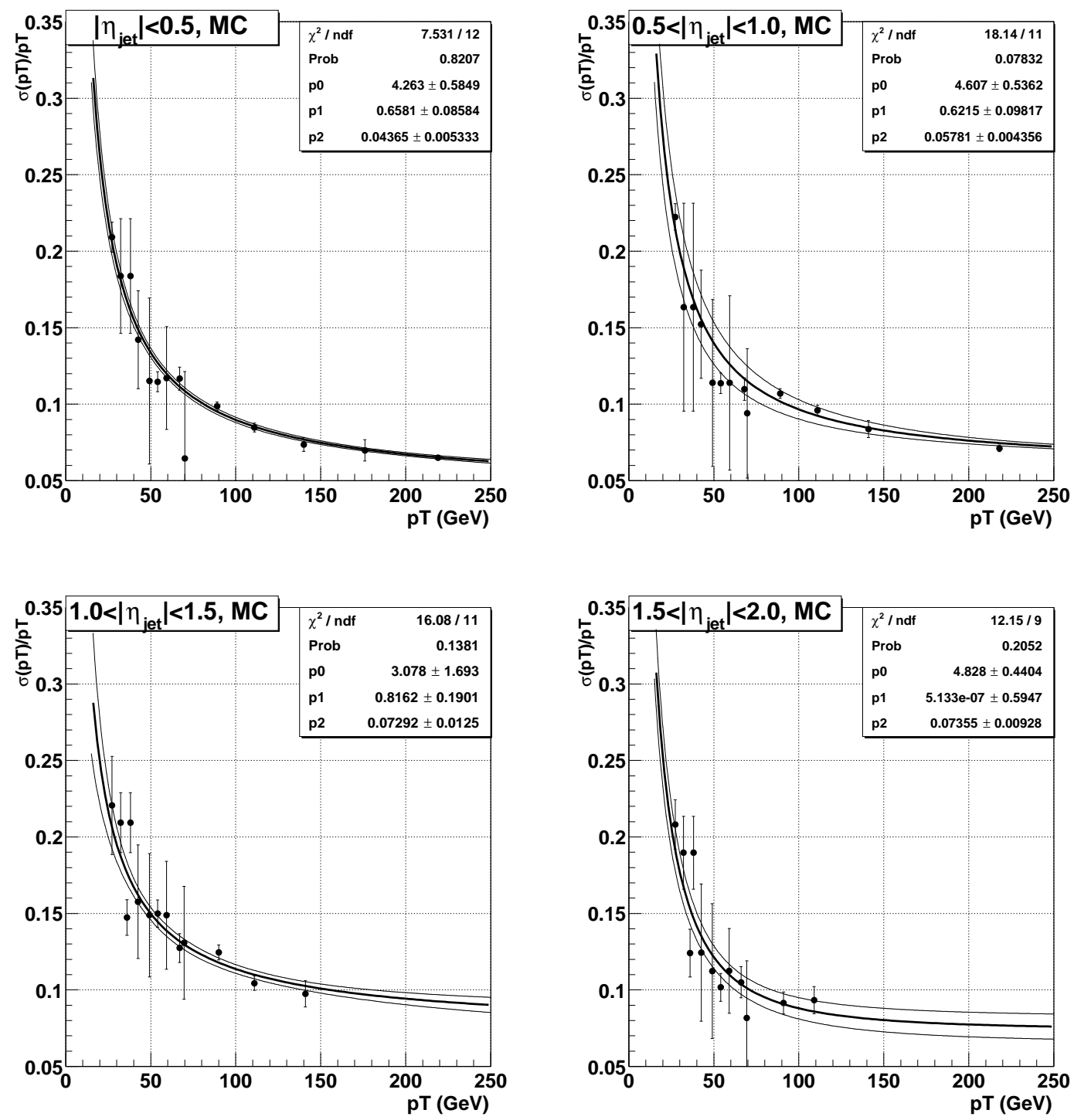

Figure 4.11: Jet $p_{T}$ resolutions for different $\eta_{\text {det }}$ regions in MC. The points below $\sim 50 \mathrm{GeV}$ are obtained using $\gamma+$ jet events, whereas for $p_{T}>50 \mathrm{GeV}$ resolutions are measured using dijet data. Bands of $\pm 1 \sigma$ statistical error are also shown. 

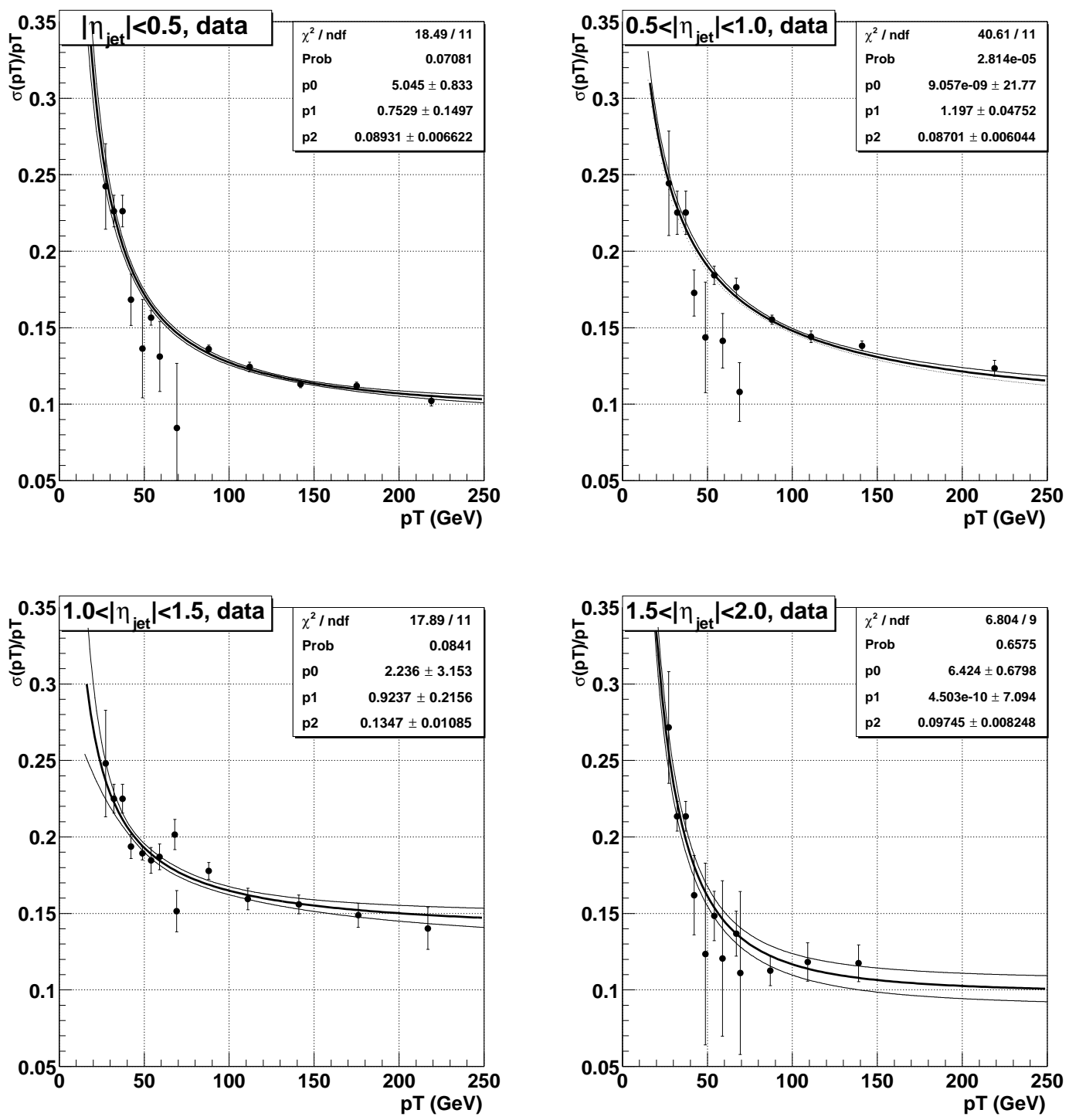

Figure 4.12: Jet $p_{T}$ resolutions for different $\eta_{\text {det }}$ regions in data. The points below $\sim 50 \mathrm{GeV}$ are obtained using $\gamma+$ jet events, whereas for $p_{T}>50 \mathrm{GeV}$ resolutions are measured using dijet data. Bands of $\pm 1 \sigma$ statistical error are also shown. 
Further details regarding the electron, muon or jet smearing can be found in Ref. [86, 87]. 


\section{Chapter 5}

\section{Analysis}

Events which pass the $e \mu$ trigger (described in Sec. 4.2) are reconstructed and stored in tapes. The size of the dataset remains quite large even at this stage and $t \bar{t} \rightarrow e \mu$ signal events are suppressed by QCD multi-jet events and events with $\mathrm{W}^{ \pm} / \mathrm{Z}^{0}$ decays. To select the events our interest, the dataset is "skimmed" by applying more stringent selection criteria and only the events with one reconstructed electron and one reconstructed muon (both with $p_{T}>$ $15 \mathrm{GeV}$ ) are selected. The analysis presented in this dissertation uses such a skimmed dataset called the "EMU" skim. Further selection criteria are applied to these skimmed events to select the $t \bar{t} \rightarrow e \mu$ events. In this chapter, the description of such selection criteria, estimation of expected number of signal and background events after applying the selection criteria, and the 
evaluation of $t \bar{t}$ production cross section are presented.

\subsection{Event Selection}

The fundamental signature of $t \bar{t} \rightarrow e \mu$ decays consists of two well-identified, high $p_{T}$ isolated leptons (one electron and one muon). The events containing one electron and one muon are dominated by $Z^{0} / \gamma^{*} \rightarrow \tau^{+} \tau^{-} \rightarrow e \mu$ events. The spatial separation (in $\eta-\phi$ space) between the electron and the muon, $\Delta R(e, \mu)^{\S}$ has been chosen to be $>0.25$, to reject the events in which, a bremsstrahlung photon emitted by the muon in the detector, appears to be an electron candidate. Basically two types of events viz., $W^{ \pm} \rightarrow \mu \nu_{\mu}$ and $Z^{0} / \gamma^{*} \rightarrow \mu^{+} \mu^{-}$can reproduce such signature discussed later in Sec. 5.5.2.

Apart from the leptons, $t \bar{t} \rightarrow e \mu$ decays also contain two highly energetic jets from the decays of $\mathrm{b}$ quarks. This feature provides a powerful discrimination against $W^{+} W^{-} \rightarrow e \mu$ and $Z^{0} / \gamma^{*} \rightarrow \tau^{+} \tau^{-} \rightarrow e \mu$ decays. Selecting events with high $\mathbb{E}_{\mathrm{T}}$ substantially reduces the remaining $Z^{0} / \gamma^{*} \rightarrow \tau^{+} \tau^{-}$ and QCD backgrounds. Additional topological selection criteria further improve the statistical power for the cross section measurement. One such

\footnotetext{
$\S \Delta R(e, \mu)=\sqrt{\left(\eta_{\text {electron }}-\eta_{\text {muon }}\right)^{2}+\left(\phi_{\text {electron }}-\phi_{\text {muon }}\right)^{2}}$ i.e., the distance in $\eta-\phi$ space between the electron candidate and any muon in the event.
} 
topological variable called $H_{T}^{\text {leading lepton }}$, which is the sum of jet $p_{T}$ 's and leading lepton $p_{T}$, has been used in this analysis to provide rejection for almost all the backgrounds.

Several other topological variables that might aid in rejection of primary background decays have been investigated. For example, jets produced for all the background processes arise from strong interactions which provide a significant tendency for the event to be arranged in one plane. Jets in top quark events, on the other hand, arise from the decay of a very massive particle and are arranged more uniformly in three dimensions. Therefore, topological variables like aplanarity $(\mathcal{A})$ and sphericity $(\mathcal{S})$, defined in Appendix A, would also be helpful to improve the signal-to-background ratio (S/B). The $Z^{0} / \gamma^{*} \rightarrow \tau^{+} \tau^{-}$events have some distinct properties that could be used for discrimination. The neutrinos tend to be tightly aligned with the charged leptons from $\tau^{\prime}$ 's and an effective invariant mass for the $\tau^{+} \tau^{-}$ system might be computed (see Appendix A). Unfortunately, neither the event shape variables $(\mathcal{A}$ and $\mathcal{S})$, nor the $\tau$ mass variable have been found to improve $\mathrm{S} / \mathrm{B}$ ratio for this analysis and therefore are not used here.

Angular correlation between the leptons and Missing $E_{T}$ has been studied. The cut, $\Delta \phi\left(\mu, \mathrm{E}_{\mathrm{T}}\right)>0.25$ provides an extra $25 \%$ rejection for the $Z^{0} / \gamma^{*} \rightarrow \tau^{+} \tau^{-}$decays. A mis-measurement of muon transverse momentum 
can lead to an incorrect measurement of $\mathrm{E}_{\mathrm{T}}$ of an event. If the measured muon momentum is less than the actual muon momentum, the estimated $\mathrm{E}_{\mathrm{T}}$ of the event will be less than the actual value. Moreover, since $\tilde{E}_{T}=-\tilde{E}_{T}^{C a l}-\tilde{p}_{T}^{\mu}$ (see Sec. 3.2.6), it will be misaligned and point close to the muon direction. Fig. 5.1 shows the distributions in $\Delta \phi\left(\mu, \mathrm{E}_{\mathrm{T}}\right)$ vs $\mathrm{E}_{\mathrm{T}}$ for $t \bar{t}, W^{+} W^{-}$, fake backgrounds and $Z^{0} / \gamma^{*} \rightarrow \tau \tau$ backgrounds for inclusive events containing zero or more jets. The same distributions are also shown in Fig. 5.2 when the events pass all the selection criteria (see Tab. 5.1), except those on $\Delta \phi\left(\mu, \mathrm{E}_{\mathrm{T}}\right)$ and $E_{T}$. It is to be noted that the back-to-back alignment between $E_{T}$ and $\mu$ can also be the indication for mis-measurement of muon $p_{T}$ (and thus $\mathbb{E}_{\mathrm{T}}$ ) where the actual muon momentum is lower than the measured one. But, as supported by Figs. 5.1 and 5.2, applying a cut to reject the events where $\mu$ and $\mathrm{E}_{\mathrm{T}}$ are back-to-back, does not provide significantly extra discrimination over the backgrounds. It is to be mentioned here that the angular correlation between the electron and the $\mathrm{E}_{\mathrm{T}}$ has also been studied but does not improve $\mathrm{S} / \mathrm{B}$ and thus is not applied in this analysis. The set of selection criteria applied in this analysis is presented in Tab. 5.1. 


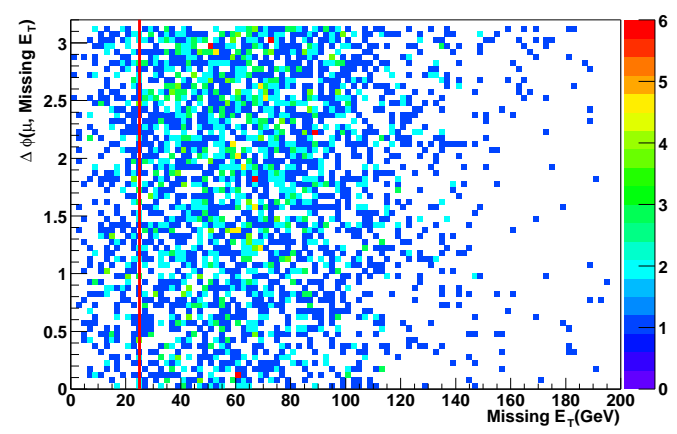

(a)

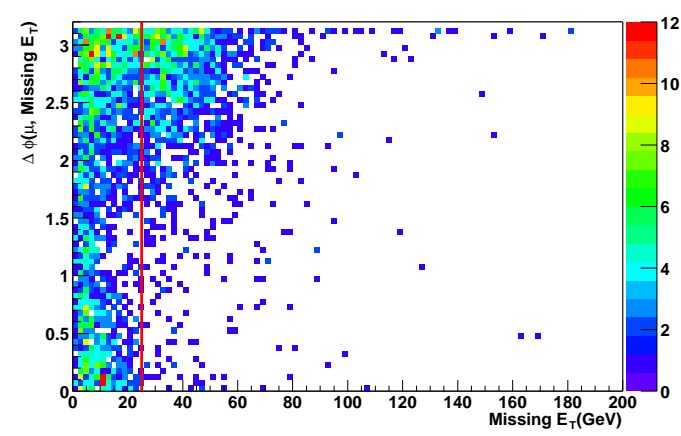

(c)

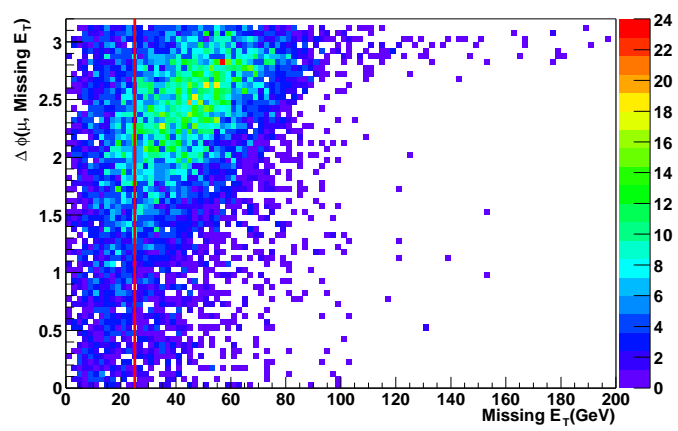

(b)

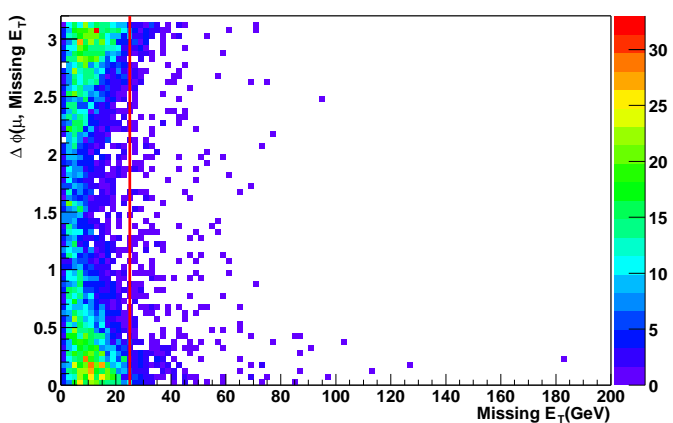

(d)

Figure 5.1: $\Delta \phi\left(\mu, \mathrm{E}_{\mathrm{T}}\right)$ vs $\mathrm{E}_{\mathrm{T}}$ distributions after $e, \mu$ selection and $\Delta R(e, \mu)>$ 0.25 cut (events with zero or more jets). Relative event density is shown in colours: (a) $t \bar{t}$, (b) $W^{+} W^{-}$, (c) fakes and (d) $Z^{0} / \gamma^{*} \rightarrow \tau^{+} \tau^{-}$. For $t \bar{t}$, the plot is equally populated, while the distributions are non-uniform for the background channels. $Z^{0} / \gamma^{*} \rightarrow \tau^{+} \tau^{-}$events produce significantly softer $\mathrm{E}_{\mathrm{T}}$ spectrum as expected. 


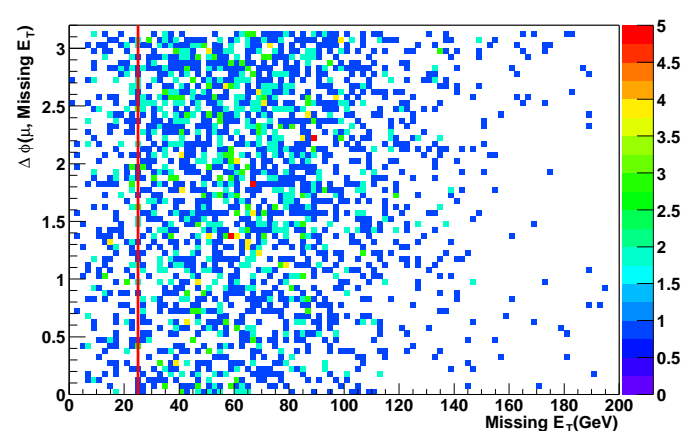

(a)

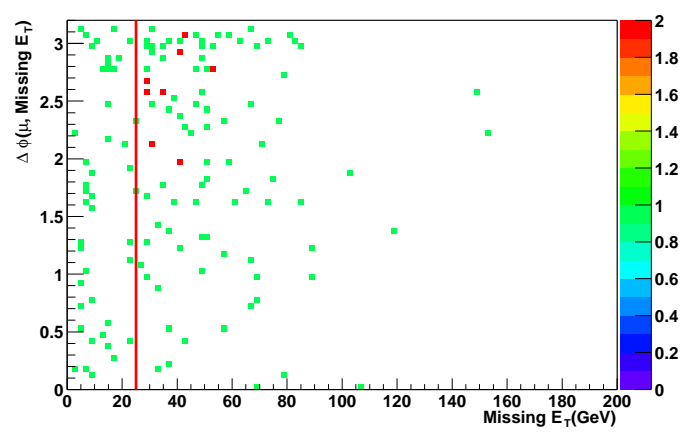

(c)

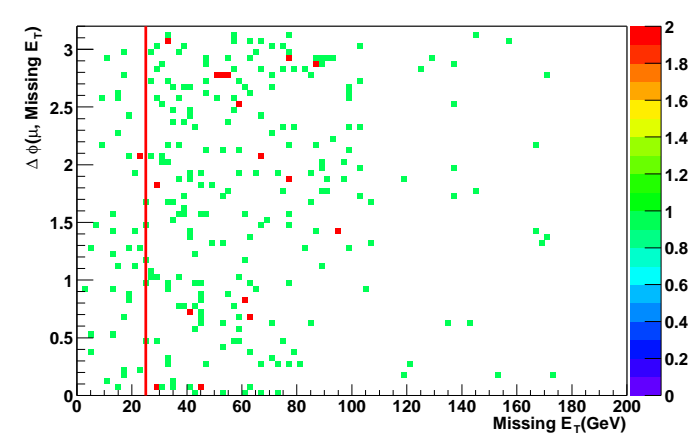

(b)

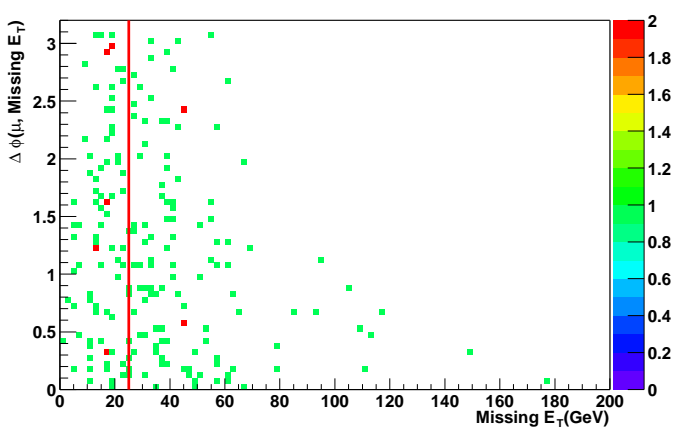

(d)

Figure 5.2: $\Delta \phi\left(\mu, E_{\mathrm{T}}\right)$ vs $\mathrm{E}_{\mathrm{T}}$ distributions after applying all all cuts: (a) $t \bar{t}$, (b) $W^{+} W^{-}$, (c) fakes and (d) $Z^{0} / \gamma^{*} \rightarrow \tau^{+} \tau^{-}$. $t \bar{t}, W^{+} W^{-}$and fake distributions are quite uniform while for a significant fraction of $Z^{0} / \gamma^{*} \rightarrow$ $\tau^{+} \tau^{-}$events, the $\mathrm{E}_{\mathrm{T}}$ direction is close to the muon direction. 


\begin{tabular}{l|c}
\hline \hline cut & role \\
\hline $\begin{array}{l}2 \text { tight, high } p_{T}(>15 \mathrm{GeV}), \\
\text { isolated leptons }(e \text { and } \mu)\end{array}$ & initial selection \\
\hline$+\Delta R(e, \mu)>0.25$ & $\left(W^{ \pm} \rightarrow \mu \nu_{\mu}\right.$ and $\left.Z^{0} / \gamma^{*} \rightarrow \mu^{+} \mu^{-}\right)$ \\
\hline+2 jets with $p_{T}(>20 \mathrm{GeV})$ jet & primarily $W^{+} W^{-}$and $Z^{0} / \gamma^{*} \rightarrow \tau^{+} \tau^{-}$rejection \\
\hline+ Missing $E_{T}>25 \mathrm{GeV}$ & $Z^{0} / \gamma^{*} \rightarrow \tau^{+} \tau^{-} \&$ QCD-multijet events removal \\
\hline$+H_{T}^{\text {eeading lepton }}>140 \mathrm{GeV}$ & further removal of all remaining backgrounds \\
\hline$+\Delta \phi\left(\mu, \mathrm{E}_{\mathrm{T}}\right)>0.25$ & Additional $Z^{0} / \gamma^{*} \rightarrow \tau^{+} \tau^{-}$rejection \\
\hline \hline
\end{tabular}

Table 5.1: Event selection criteria for rejecting different background processes.

\subsection{Optimization of Cuts}

A cross section measurement is sensitive to the choice of selection criteria.

Selection criteria based on the topological variables listed in Tab. 5.1 (i.e., $\mathrm{E}_{\mathrm{T}}^{\prime}, H_{T}^{\text {leading lepton }}$ and $\Delta \phi\left(\mu, \mathrm{E}_{\mathrm{T}}\right)$ have been optimized such that, the relative statistical error on cross section measurement i.e., $\Delta \sigma_{\text {stat }} / \sigma$ is the smallest.

\subsubsection{Figure of Merit}

The relative error on cross section measurement can be written as (see Appendix B)

$$
\frac{\Delta \sigma}{\sigma}=\frac{\sqrt{S+B}}{S} \bigoplus \frac{\Delta B_{\text {syst }}}{S} \bigoplus \frac{\Delta S_{\text {syst }}}{S}
$$


where $S, B$ are the expected number of signal and background events and $\Delta S_{\text {syst }}, \Delta B_{\text {syst }}$ are the systematic uncertainties in $\mathrm{S}$ and $\mathrm{B}$ respectively. The first term on right hand side of Eqn. 5.1 is the relative statistical uncertainty, while the combination of the other two terms lead to relative systematic uncertainty on the cross section measurement. Since $t \bar{t}$ events are rare at the current luminosities of Tevatron, statistical error on $t \bar{t}$ cross section measurement is the dominating one. Hence, $\sqrt{S+B} / S$ is chosen to be the figure of merit (FOM) while optimizing the selection criteria. In other words, the lowest value of $\sqrt{S+B} / S$ would indicate an optimal choice of the cuts.

\subsubsection{Grid Search}

In order to find an optimal set of cuts, the events containing

- One track-matched electron with $p_{T}>15 \mathrm{GeV}$, passing the likelihood cut and EM ID criteria (see Sec. 3.2.1)

- One track-matched isolated muon with $p_{T}>15 \mathrm{GeV}$ (see Sec. 3.2.3)

- At least two jets with $E_{T}>20 \mathrm{GeV}$, passing the Jet selection criteria (see Sec. 3.2.4)

are preselected first. In a grid search, one simultaneously varies all the topological variables under consideration and for all possible combinations of the 
cut values the number of signal events and number of background events, are estimated. The FOM is calculated for each combination and the values of cuts on the topological variables which correspond to the lowest value of FOM is chosen as the best set of cuts.

As mentioned earlier, very few topological variables are found to be efficient in background rejection. Apart from the $\mathrm{E}_{\mathrm{T}}^{\prime}$ cut and $\Delta \phi\left(\mu, \mathrm{E}_{\mathrm{T}}\right)$ cut, the $H_{T}^{\text {leading lepton }}$ variable (which is the sum of the $E_{T}$ 's of the jets and $p_{T}$ of the leading lepton) is found to be quite effective. At first, trials have been made to choose the best $\mathrm{H}_{\mathrm{T}}$ variable based on the jet and lepton $p_{T}$ 's. Four such variables are:

- $H_{T}^{\mathrm{jets}}=\sum_{\mathrm{jets}} p_{T}^{\mathrm{jet}}$

- $H_{T}^{\text {leading lepton }}=\sum_{\text {jets }} p_{T}^{\text {jet }}+p_{T}^{\text {leading lepton }}$

- $H_{T}^{\text {electron }}=\sum_{\text {jets }} p_{T}^{\text {jet }}+p_{T}^{\text {electron }}$

- $H_{T}^{\text {Both Lepton }}=\sum_{\text {jets }} p_{T}^{\text {jet }}+\sum_{\text {lepton }} p_{T}^{\text {lepton }}$

In order to choose a particular definition of $H_{T}$, a two dimensional grid search (in $\mathrm{E}_{\mathrm{T}}$ and various definitions of $H_{T}$ ) is performed. It has been found that the $H_{T}^{\text {leading lepton }}>140 \mathrm{GeV}$ along with $\mathrm{E}_{\mathrm{T}}>25 \mathrm{GeV}$ provides an optimal solution [88]. 


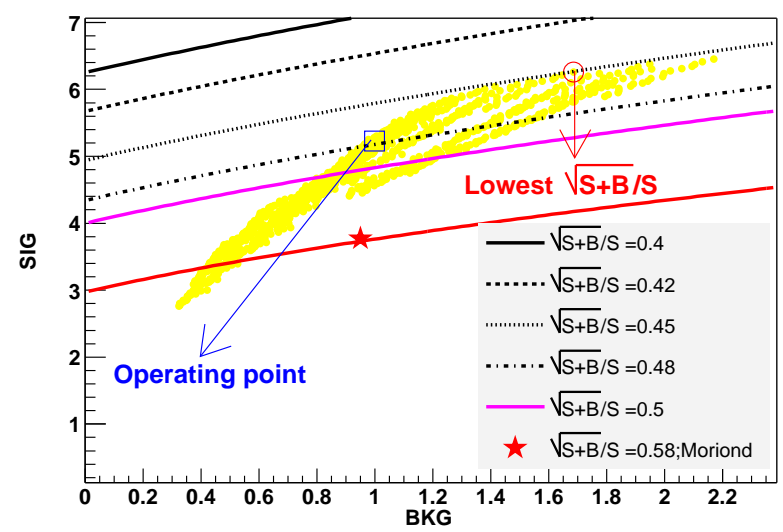

Figure 5.3: Values of number of signal $(S)$ vs. number of expected background $(B)$ in grid search (shown in yellow marker). Contours with $\sqrt{S+B} / S$ is shown. Best choice is indicated by "Lowest $\sqrt{S+B} / S$ ", while the "Operating point" corresponds to the cuts tabulated in Tab. 5.1. The sensitivity of the previous version of this analysis [89] is also shown and referred to as "Moriond".

Then in a 3-dimensional grid search (with variables $\mathbb{E}_{\mathrm{T}}, H_{T}^{\text {leading lepton }}$ and $\left.\Delta \phi\left(\mu, \mathrm{E}_{\mathrm{T}}\right)\right)$ is performed where the $\mathrm{E}_{\mathrm{T}}$, and $\mathrm{H}_{\mathrm{T}}^{\text {Leading lepton }}$ are varied in steps of 5 and $10 \mathrm{GeV}$ respectively, while the $\Delta \phi\left(\mu, \mathrm{E}_{\mathrm{T}}\right)$ has been varied in steps of 0.05. The results of this grid search are shown in Fig. 5.3 and the constant contours for different values of $\frac{\sqrt{S+B}}{S}$ have been overlaid.

As shown in Fig. 5.3, the best FOM $(=\sqrt{S+B} / S)$ is found to be 0.46 with a set of cuts $\left(\mathbb{E}_{\mathrm{T}}>20 \mathrm{GeV}, \mathrm{H}_{\mathrm{T}}^{\text {leading lepton }}>120 \mathrm{GeV}\right.$ and $\left.\Delta \phi\left(\mu, \mathrm{E}_{\mathrm{T}}\right)>0.75\right)$. The combination of cuts presented in Tab. 5.1 (i.e., $\mathrm{E}_{\mathrm{T}}>25 \mathrm{GeV}, \mathrm{H}_{\mathrm{T}}^{\text {leading lepton }}>140 \mathrm{GeV}$ and $\Delta \phi\left(\mu, \mathrm{E}_{\mathrm{T}}\right)>0.25$, pointed 


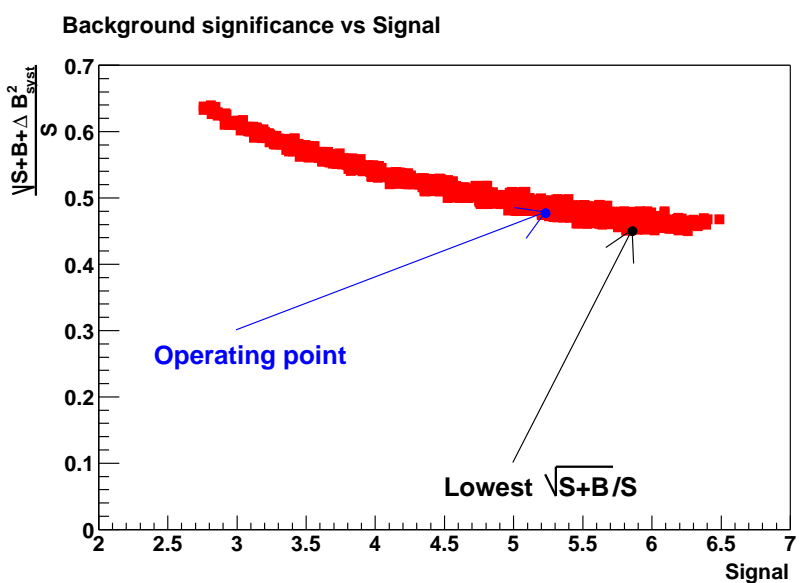

Figure 5.4: Variation of $\sqrt{S+B+\Delta B_{\text {syst }}^{2}} / S$ with expected number of signal $(S)$ events.

to as "Operating point" in Fig. 5.3) gives a value of $\mathrm{FOM}=0.48$, but with background contribution lower by $1 / 3$ than that for the set of cuts which corresponds to the best FOM. To justify the "Operating point" further, the values of $\Delta B_{\text {syst }} / S$ are close for both sets of cuts. Fig. 5.4 shows the variation of modified FOM $\left(=\sqrt{S+B} / S \bigoplus \Delta B_{\text {syst }} / S\right)$ as a function of expected number of signal events.

\subsection{Data to MC Scale factors}

To take into account the discrepancies in modelling of the $\mathrm{D} \varnothing$ detector in $\mathrm{d \emptyset gstar}$ code, the distributions of the kinematic variables in the Monte Carlo 
are smeared (see Sec. 4.3.3). Even after smearing, the rates at which Monte Carlo events pass various selection criteria do not match with corresponding rates in data. Scale factors for different selection criteria are estimated to make these rates identical for data and MC events. Such a scale factor can be defined as

$$
\kappa=\frac{\epsilon^{\mathrm{data}}}{\epsilon^{\mathrm{MC}}}
$$

where $\epsilon^{\mathrm{MC}}\left(\epsilon^{\text {Data }}\right)$ is the efficiency of a particular selection criterion, measured in Monte Carlo (Data) events.

\subsubsection{PV Scale Factors}

Primary vertices are reconstructed in the p14 version of D $\varnothing$ event reconstruction package, using two vertexing algorithms: dØreco and dØroot (discussed earlier in Sec. 3.1.2). The current analysis uses the dØroot version of the vertex finder for reconstructing the primary vertex in an event, but also checks the consistency of the position of PV with dØreco algorithm by imposing a selection criterion of $\mid z_{\mathrm{PV}}(\mathrm{d} \emptyset$ root $)-z_{\mathrm{PV}}(\mathrm{d} \emptyset$ reco $) \mid<5 \mathrm{~cm}$.

In order to ensure high quality of the reconstructed vertex, it is required to be within the SMT fiducial region $\left(\left|z_{\mathrm{PV}}\right| \leq 60 \mathrm{~cm}\right)$ and have at least three tracks attached to it. The leptons in a $t \bar{t} \rightarrow e \mu$ event are prompt and in 
principle, the tracks associated with the leptons should pass through the primary vertex. This feature of the $t \bar{t}$ decays has been checked by imposing a cut on the distance between the impact parameter of each lepton track with respect to the primary vertex in the z-direction, $\mid \Delta z($ lepton, PV) $\mid<1$ $\mathrm{cm}$.

The efficiencies for the primary vertex selection criteria for $\mathrm{MC}$ are evaluated using $\left(Z^{0} / \gamma^{*} \rightarrow l l\right) j$ and $\left(Z^{0} / \gamma^{*} \rightarrow l l\right) j j(1=\mathrm{e}, \mu)$ Alpgen Monte Carlo samples, while the same efficiencies are estimated using $Z^{0} / \gamma^{*} \rightarrow \mu \mu$ and $Z^{0} / \gamma^{*} \rightarrow$ ee decays in data. Tab. 5.2 presents these efficiencies, along with the scale factors.

The scale factors for the PV selection criteria " $\left|\mathrm{z}_{\mathrm{PV}}\right|<60 \mathrm{~cm}$ AND N $\mathrm{N}_{\text {trk }} \geq$ 3", show very slight dependence on the number of jets, in both $Z^{0} / \gamma^{*} \rightarrow e e$ and $Z^{0} / \gamma^{*} \rightarrow \mu \mu$ cases. The values of inclusive ( $\geq 0$ jet) measurements are $1 \%$ lower than the corrsponding values obtained from $\geq 1$ jet (or $\geq 2$ jets) measurements. The differences in these efficiencies are considered as the systematic uncertainties in the scale factors for " $\left|z_{\mathrm{PV}}\right|<60 \mathrm{~cm} \mathrm{AND} \mathrm{N}_{\text {trk }} \geq$ 3" selection criteria. Data efficiency for the selection criterion based on $\mid z_{\mathrm{PV}}(\mathrm{d} \emptyset$ root $)-z_{\mathrm{PV}}(\mathrm{d} \emptyset \mathrm{reco}) \mid<5 \mathrm{~cm}$, is very well represented by the Monte Carlo efficiency for both $Z^{0} / \gamma^{*} \rightarrow e e$ and $Z^{0} / \gamma^{*} \rightarrow \mu \mu$ processes. The $\Delta z$ (PV,lepton) scale factor is a constant within errors for $Z^{0} / \gamma^{*} \rightarrow e e$ and 


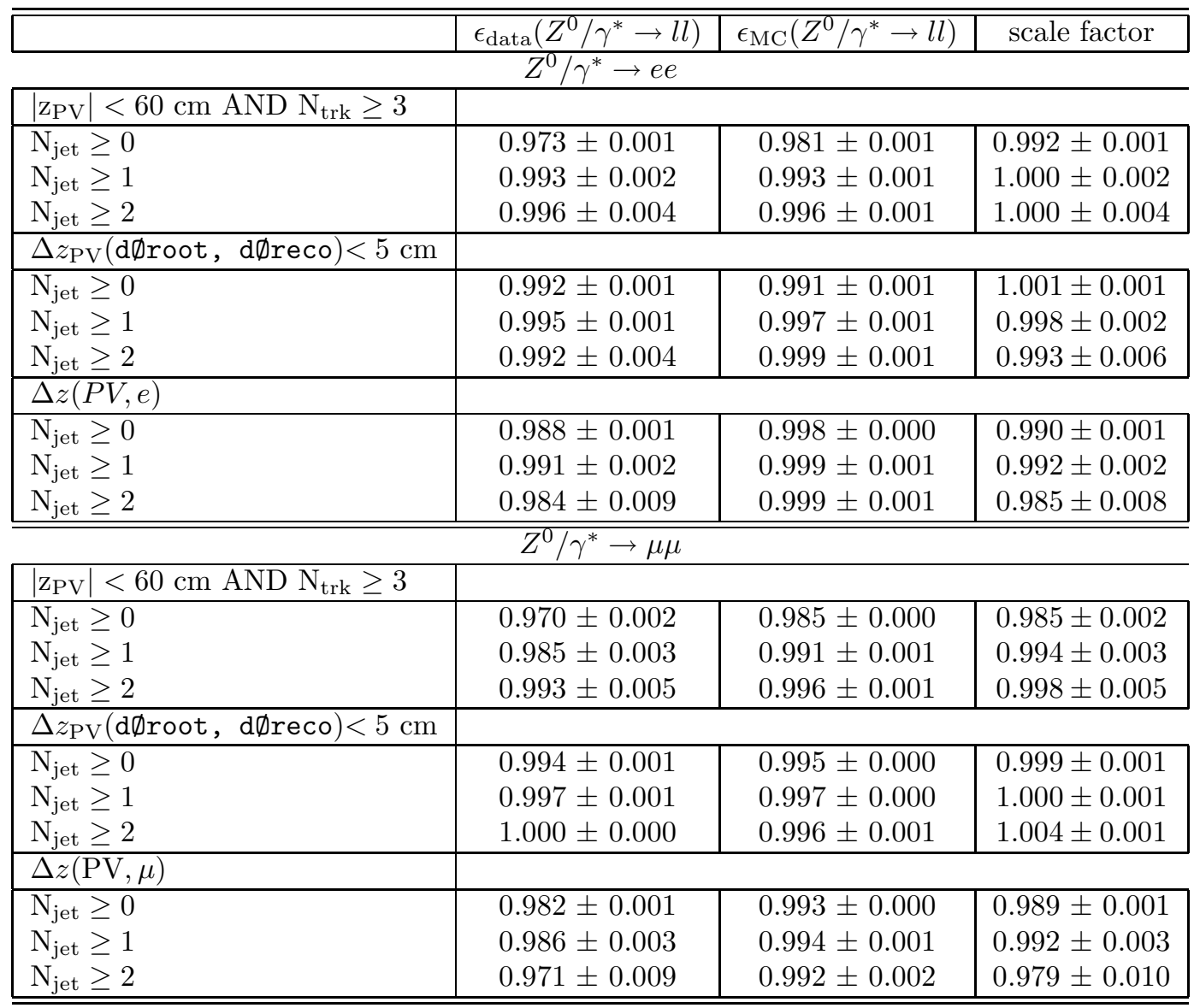

Table 5.2: Primary vertex cuts efficiencies (in \%) in $Z^{0} / \gamma^{*} \rightarrow e e$ and $Z^{0} / \gamma^{*} \rightarrow \mu \mu$ for data and $\mathrm{MC}$ and a scale factor as a function of jet multiplicity. All uncertainties are statistical. 


\begin{tabular}{|l|c|}
\hline \hline & scale factor \\
\hline$\kappa^{\mathrm{PV}}\left(\mathrm{N}_{\text {jet }} \geq 0\right)$ & $0.989 \pm 0.002($ stat $) \pm 0.007($ sys $)$ \\
$\kappa^{\mathrm{PV}}\left(\mathrm{N}_{\text {jet }} \geq 1, \mathrm{~N}_{\text {jet }} \geq 2\right)$ & $0.997 \pm 0.003($ stat $)$ \\
$\kappa^{\Delta z(d \varnothing \text { reco, }, \varnothing \text { root })}$ & $1.000 \pm 0.001($ stat $)$ \\
$\kappa^{\Delta \mathrm{z}(\mathrm{PV}, \mathrm{e} / \mu)}$ & $0.990 \pm 0.001($ sys $)$ \\
\hline \hline
\end{tabular}

Table 5.3: Scale factors related to primary vertices derived from Tab. 5.2. Statistical errors are taken as the uncorrelated sum of the statistical errors from the $Z^{0} / \gamma^{*} \rightarrow e e$ and $Z^{0} / \gamma^{*} \rightarrow \mu \mu$ samples.

$Z^{0} / \gamma^{*} \rightarrow \mu \mu$ decays, and does not vary with the jet multiplicity. The current analysis takes the average between $Z^{0} / \gamma^{*} \rightarrow e e$ and $Z^{0} / \gamma^{*} \rightarrow \mu \mu$ scale factors which are provided in Tab. 5.3. When there are statistically significant differences between $Z^{0} / \gamma^{*} \rightarrow e e$ and $Z^{0} / \gamma^{*} \rightarrow \mu \mu$ primary vertex scale factors, the corresponding differences are taken into account during the calculation of systematic uncertainties.

\subsubsection{Scale Factors for Electron selection criteria}

This analysis accepts the candidate electromagnetic (EM) clusters, reconstructed in the central calorimeter $\left(\mathrm{CC},\left|\eta_{\text {det }}\right|<1.1\right)$ or endcap calorimeter (EC, $\left.1.5<\left|\eta_{\text {det }}\right|<2.5\right)$. Three criteria are considered for electron identification: electromagnetic cluster identification, track match, and likelihood criteria (see Sec. 3.2.1). For electrons, the CC and EC regions are treated 
separately because of the differences in calorimeter layers and amount of upstream detector material in these regions. Medium and tight electrons (see Sec. 3.2.1 for these definitions of an electron), are considered to estimate the scale factors associated with different selection criteria for electrons, while a "photon" is considered as a medium electron which does not have a matching track in a $0.05 \times 0.05$ road in $\eta-\phi$ around the cluster.

The efficiency for an electron to pass all criteria for a tight electron is divided into three parts. The first part is the efficiency for an electron to be reconstructed as an EM cluster and pass all identification criteria that makes it a medium electron. This efficiency will be referred to as the "electron reconstruction and identification" efficiency $\left(\epsilon_{\mathrm{ID}}^{\mathrm{EM}}\right)$. The second part is the probability for a medium electron to be matched to a track. This is referred to as the track match efficiency $\left(\epsilon_{\mathrm{trk}}^{\mathrm{EM}}\right)$. Finally, the probability for a medium electron with a track match to pass the electron likelihood is called the likelihood efficiency $\left(\epsilon_{\text {lhood }}^{E M}\right)$. The division into three parts reflects the way in which the efficiencies are derived.

\section{Medium Electron Efficiency}

The efficiency to pass the medium electron criteria is determined using $Z^{0} / \gamma^{*} \rightarrow$ ee data and MC samples where each event contains a tight electron matched 

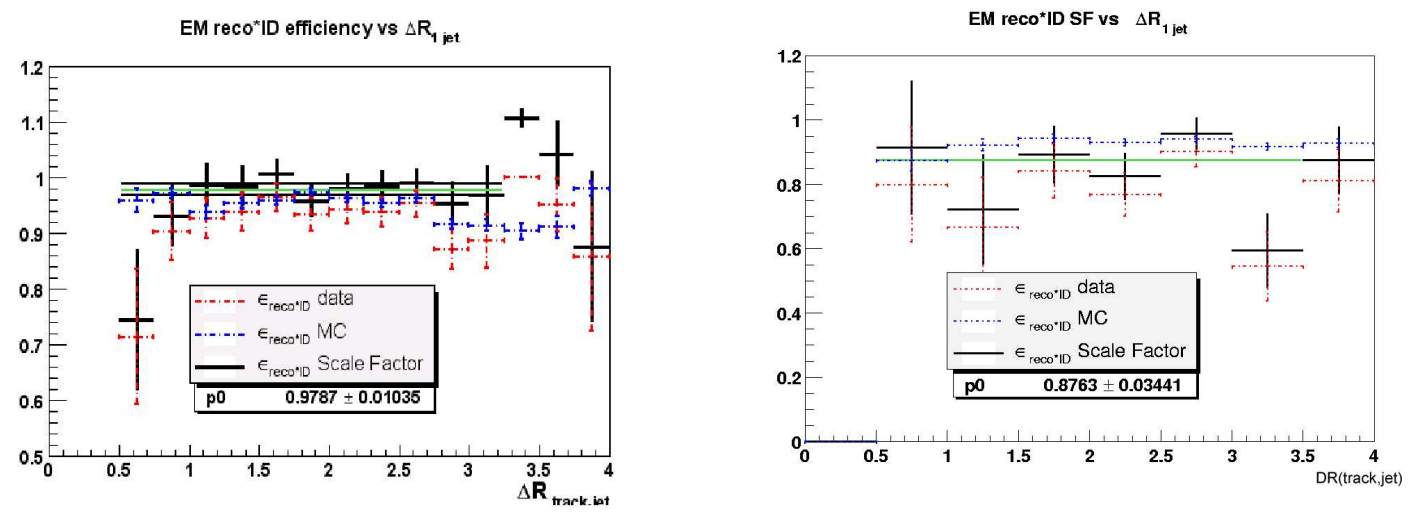

Figure 5.5: EM reconstruction and identification efficiencies in data and Monte Carlo and the corresponding scale factors as function of the distance between the electron track and the closest jet in CC (left) and EC (right).

to a central track and a second central track (the probe track). The events are further cleaned up by requiring the invariant mass of the two central tracks to be within $\pm 20 \mathrm{GeV}$ of the $Z^{0}$ boson mass. The rate at which the probe track is matched to a reconstructed medium electron cluster, allows to derive the efficiency for medium electron selection in data and MC samples.

The scale factor for the medium electron efficiency is determined as a function of the distance between the electron and the closest jet in the event, $\Delta R(e$, jet $)$, in events with only one jet, as a check for possible systematic effects due to electron-jet overlap. This scale factor is shown for CC and EC in Fig. 5.5 and shows no statistically significant dependence. The systematic uncertainty on this scale factor is obtained from the scatter (RMS) of the 


\begin{tabular}{|l||c|c|}
\hline \hline \multicolumn{1}{|c||}{ Selection Criteria } & \multicolumn{2}{c|}{ Scale factor } \\
\cline { 2 - 3 } & CC & EC \\
\hline Medium electron & $0.979 \pm 0.026$ (syst) & $0.876 \pm 0.067$ (syst) \\
Likelihood + Track matching & $0.869 \pm 0.018$ (syst) & $0.753 \pm 0.033$ (syst) \\
Track matching & $0.946 \pm 0.002$ (stat) & $0.782 \pm 0.005$ (stat) \\
\hline \hline
\end{tabular}

Table 5.4: Data-to-MC scale factors for the medium electron efficiency, the track match times likelihood efficiency and for the track match efficiency. The systematic uncertainties associated to each scale factor are also given.

scale factor versus $\Delta R(e$, jet $)$ in Fig. 5.5. The resulting scale factors and systematic uncertainties are given in Tab. 5.4.

\section{Track match and Likelihood Efficiency}

The electron track match probability is derived from data and MC samples containing $Z^{0} / \gamma^{*} \rightarrow e e$ events with one tight electron matched to a central track and a second medium electron, called probe electron, having an invariant mass within $\pm 20 \mathrm{GeV}$ of the $Z^{0}$ boson mass (calculated from calorimeter information). The rate at which the probe electron matches to a central track is the electron track matching probability. Finally, the efficiency for the likelihood selection is derived from data and MC samples of $Z^{0} / \gamma^{*} \rightarrow e e$ events, with one tight electron matched to a central track and a medium electron matched to a central track. The rate at which the medium electron passes the likelihood selection gives the likelihood efficiency. 
The scale factor for the track matching times likelihood efficiency is derived as functions of $\eta$ and $\phi$ of the electron (see Fig. 5.6). For electrons in $\mathrm{CC}$, the value of the scale factor used in the analysis is obtained by fitting the scale factor versus $\eta$ with a straight line. The systematic uncertainty on this number is taken to be the largest scatter from the 2 plots showing the scale factor versus $\eta$ and versus $\phi$. The resulting scale factors and the associated systematic uncertainties are given in Tab. 5.4. For electrons in EC, the scale factor for track match times likelihood efficiency is linearly dependent on $\eta$, as shown in Fig. 5.6. Therefore to obtain a scale factor for EC electrons, the $\eta$ dependent scale factors for EC (in bin of $\eta$ ), is weighted with the $\eta$ spectra of electrons in EC from $t \bar{t} \rightarrow e e$ and $t \bar{t} \rightarrow e \mu$ Monte Carlo events. The same procedure has been applied for all background processes of this analysis. It has been found that the scale factors for EC obtained for $t \bar{t} \rightarrow e e$, $t \bar{t} \rightarrow e \mu$ and background processes, are consistent within errors. Therefore, a single scale factor for EC electrons (as obtained by combining $t \bar{t} \rightarrow e e$ and $t \bar{t} \rightarrow e \mu)$, is used for signal and background estimation of this analysis, except for the photon backgrounds. The only scale factor applicable to the photon backgrounds i.e., track match scale factor is also quoted separately in Tab. 5.4. 

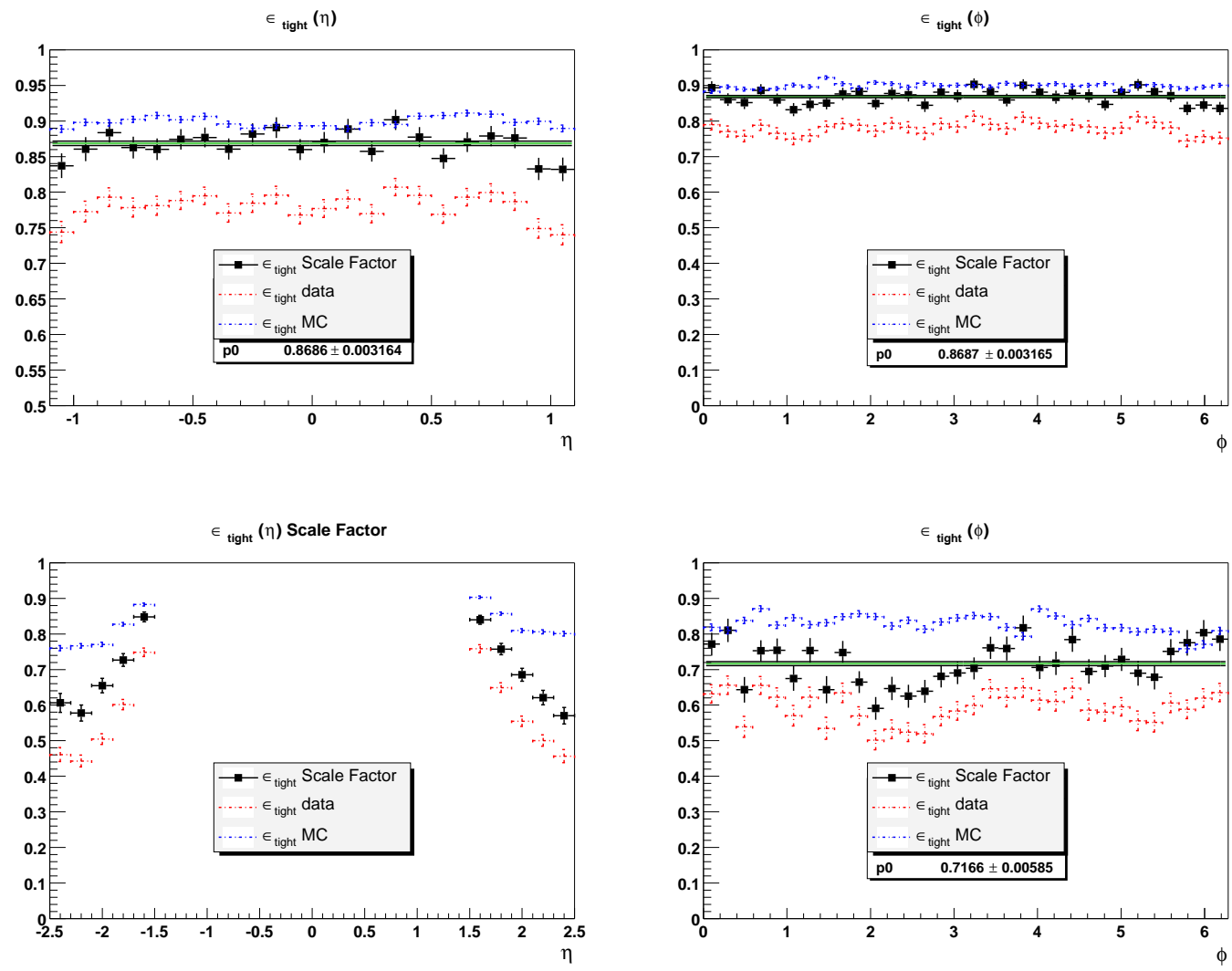

Figure 5.6: Track match times likelihood efficiency as function of $\eta$ (left plots) and $\phi$ (right plots) in CC (upper plots) and EC (lower plots). 


\subsubsection{Muon Scale Factors}

Candidate muons are accepted for this analysis if they are reconstructed in the muon system within $\left|\eta_{\text {detector }}\right|<2.0$. As mentioned earlier in Sec. 3.2.3, two types of muons (medium and tight) are used in this analysis.

\section{Muon reconstruction and identification efficiency}

To measure the efficiency of muon reconstruction and identification in data, $Z^{0} / \gamma^{*} \rightarrow \mu \mu$ events are used. These events are identified by one tight muon (control muon) and a second muon only reconstructed in the central tracking system (test muon). To ensure that the reconstructed track belongs to a muon from a $Z^{0}$ decay, the invariant mass of the control and the test muon should be within $\pm 20 \mathrm{GeV}$ of the $Z^{0}$ boson mass. The events are selected using a single muon trigger and the control muon is matched with the muon that triggered the event. The rate at which the test muon is matched to a reconstructed local muon is a measure of the local muon reconstruction efficiency.

The same procedure is applied to $Z^{0} / \gamma^{*} \rightarrow \mu \mu$ Monte Carlo events and the differential efficiencies as a function of detector $\eta, \phi$ and $p_{T}$ are compared with those obtained from the experimental data. The ratio of the 
measured efficiencies in data and Monte Carlo gives the scale factor. The scale factor is found to have dependence on the muon $p_{T}$ and the muon $\left|\eta_{\text {detector }}\right|$, and thus is parametrized using polynomials of first and of third order, respectively. These two parameterizations are combined into a twodimensional parametrization. Further details regarding this parametrization can be found in Ref. [90].

\section{Muon track match efficiency}

The efficiency to reconstruct and match a central track corresponding to a muon is determined in data and in Monte Carlo using $Z^{0} / \gamma^{*} \rightarrow \mu \mu$ samples. The exact procedure is described in Ref. [91]. A data to Monte Carlo scale factor is derived as functions of $\eta$ and $\phi$ of the muon. Dependence of this scale factor on event selections and background contamination in the $Z^{0} / \gamma^{*} \rightarrow \mu \mu$ sample are the main sources of systematic uncertainty in this efficiency. The scale factor corresponding to muon track match efficiency is found to be $0.98 \pm 0.03$ (syst)

\section{Efficiency of the DCA significance cut}

The DCA (Distance of Closest Approach) of a track is defined to the shortest distance (in $\mathrm{x}-\mathrm{y}$ plane) between the track and primary vertex in an event. 
The cut on DCA significance (DCA/error on DCA) helps to distinguish the muons coming from the PV, from those coming from the semileptonic decays of heavy flavours. The scale factor for the DCA significance cut has been measured by comparing DCA significance distributions in $Z^{0} / \gamma^{*}+$ jets data with those in Monte Carlo samples. The data events are selected with two muons $\left(p_{T}>15 \mathrm{GeV}\right)$ having invariant mass $70<M_{\mu \mu}<110 \mathrm{GeV}$, while for MC, the events are generated with an invariant mass of $60<M_{\mu \mu}<130$ $\mathrm{GeV}$. Both muons in these samples are further required to pass isolation, vertex quality, track match criteria. They also are required to have opposite charges. The rates at which the events in these samples, pass the DCA significance cut (i.e., both muons have to pass the DCA significance criteria) give the DCA significance efficiencies for data and MC. The data and MC efficiencies are measured as functions of jet multiplicity of the event and thus the scale factor becomes a function of jet multiplicity. As presented in Tab. 5.5, the scale factor corresponding to 0 jet multiplicity is different from those corresponding to 1 or 2 jet multiplicities. The scale factor for events with 1 or 2 jets are close to each other. Therefore, a single scale factor of $0.994 \pm 0.003$ per muon is used for all events with one or more jets. The per 


\begin{tabular}{|l||c|c|c|}
\hline \hline & $\epsilon^{\text {data }}$ & $\epsilon^{M C}$ & $\kappa$ \\
\hline$D C A$ significance $(\geq 0$ jet $)$ & $0.921 \pm 0.003$ & $0.895 \pm 0.001$ & $1.03 \pm 0.003$ \\
$D C A$ significance $(\geq 1$ jet $)$ & $0.957 \pm 0.005$ & $0.969 \pm 0.002$ & $0.988 \pm 0.006$ \\
$D C A$ significance $(\geq 2$ jet $)$ & $0.961 \pm 0.013$ & $0.980 \pm 0.003$ & $0.981 \pm 0.014$ \\
\hline \hline
\end{tabular}

Table 5.5: Per event efficiencies in $Z^{0} / \gamma^{*} \rightarrow \mu \mu$ data and Monte Carlo for the $D C A$ cut and the corresponding scale factors. The quoted errors are statistical only. These efficiencies are derived for muons with a transverse momentum of at least $15 \mathrm{GeV}$.

muon scale factor is derived as,

$$
\sqrt{\kappa_{\mathrm{DCA}}^{\text {event }}} \pm \frac{\sigma_{\kappa_{\mathrm{DCA}}^{\text {event }}}}{2}
$$

where $\kappa_{\mathrm{DCA}}^{\text {event }} \pm \sigma_{\kappa_{\mathrm{DCA}}^{\text {event }}}$ is the per event scale factor for DCA significance efficiency.

\section{Muon Isolation Efficiency}

The scale factor for the per muon efficiency of the muon isolation cut is estimated using a dataset with $Z^{0} / \gamma^{*}+2$ jets and an Alpgen Monte Carlo sample with $Z^{0} / \gamma^{*}+2$ jets. The selection criteria applied to these events are identical to those described in the previous paragraph, except for the DCA significance criterion which is applied in this case, while the isolation cuts viz., rattrk $<0.12$ and rat11<0.12 are omitted. 
The isolation scale factor can be determined by requiring the highest $p_{T}$ muon to be isolated and by measuring how often the second highest $p_{T}$ muon is isolated in data and Monte Carlo samples. To investigate a potential dependence of this scale factor on the distance to the nearest jet, it is determined as a function of the distance (in $\eta-\phi$ space) between the second highest $p_{T}$ muon and the closest jet in the event, $(\Delta R(\mu, j e t))$. The distributions of $\Delta R(\mu$, jet $)$ is shown in Fig. 5.7(a). The scale factor is derived as a function of $\Delta R(\mu$, jet) (see Fig. 5.8),

$$
\kappa_{\mathrm{Iso}}(\Delta \mathrm{R}(\mu, \text { jet }))=\epsilon_{\mathrm{Iso}}^{\mathrm{data}}(\Delta \mathrm{R}(\mu, \text { jet })) / \epsilon_{\mathrm{Iso}}^{\mathrm{MC}}(\Delta \mathrm{R}(\mu, \text { jet })) .
$$

The graph in Fig. 5.8 shows no dependence on the distance to the nearest jet. The results of a linear fit to the graph gives a scale factor of 1.001 \pm 0.006 . This result has been cross checked using different Monte Carlo samples $\left(Z^{0} / \gamma^{*}+1\right.$ jet $)$ and various event selection criteria to investigate the effect of the jet multiplicity and jet $p_{T}$ on the muon isolation efficiency. In all cases, the muon isolation efficiency in data is well reproduced by those obtained from the Monte Carlo. The scale factor averaged over $\Delta \mathrm{R}(\mu$, jet) is given in Tab. 5.6. For comparison the scale factor obtained for events 


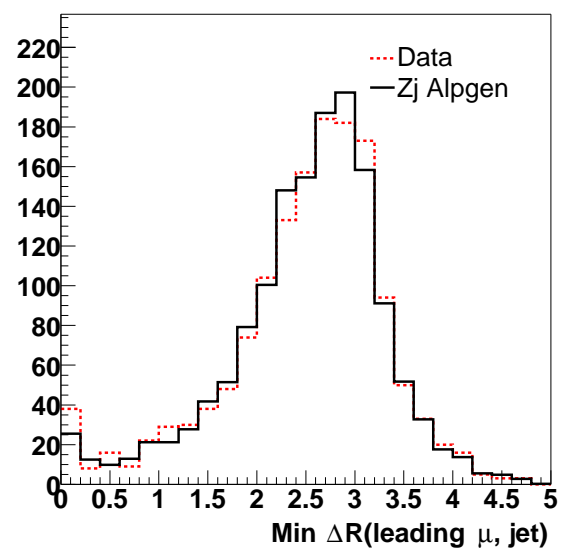

(a)

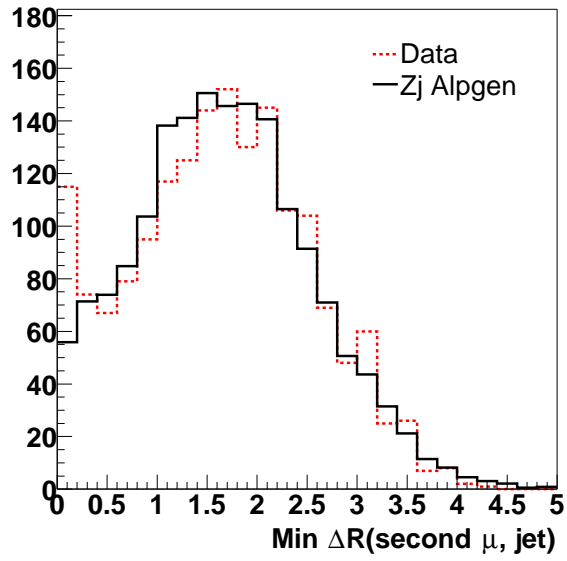

(b)

Figure 5.7: Distance between the second highest $p_{T}$ muon and the closest jet in $Z^{0} / \gamma^{*}+$ jet candidate events, when the highest $p_{T}$ muon is isolated, is shown on left for data and Monte Carlo. The right plot shows the distance between the closest jet and the highest $p_{T}$ muon when isolation selections are applied to the second highest $p_{T}$ muon.

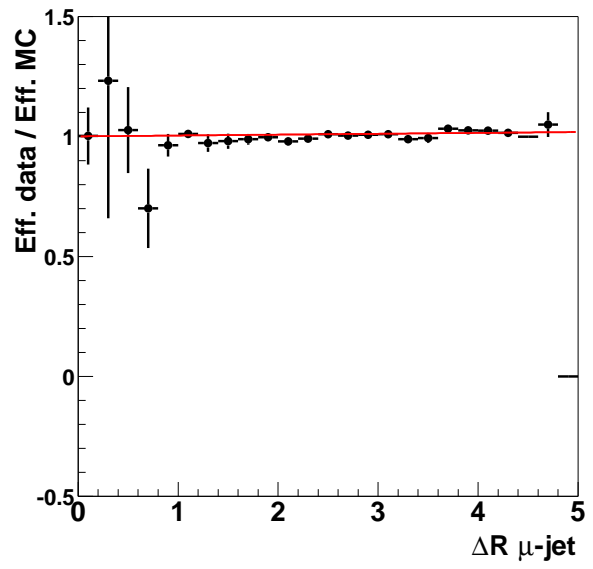

Figure 5.8: Ratio of the muon isolation efficiencies in data and Monte Carlo, measured in $Z^{0} / \gamma^{*}+$ jet events, as a function of the distance between the second highest $p_{T}$ muon and the closest jet (derived from Fig. 5.7 (a)). The result of the linear fit is a scale factor of $1.001 \pm 0.006$. 


\begin{tabular}{|l||c|c|c|}
\hline \hline & $\epsilon_{\text {Iso }}^{\text {data }}$ & $\epsilon_{I s o}^{M C}$ & $\kappa_{\text {Iso }}$ \\
\hline Isolation efficiency & $0.981 \pm 0.005$ & $0.973 \pm 0.001$ & $1.008 \pm 0.006$ \\
Isolation efficiency, $\Delta R(\mu, j e t)>0.5$ & $0.993 \pm 0.003$ & $0.992 \pm 0.001$ & $1.001 \pm 0.004$ \\
\hline \hline
\end{tabular}

Table 5.6: Per muon efficiencies in $Z^{0} / \gamma^{*} \rightarrow \mu \mu$ data and Monte Carlo samples for the muon isolation cut and the corresponding scale factor. The isolation efficiency with the additional requirement $\Delta R(\mu, j e t)>0.5$ is provided as a cross check. The quoted errors are statistical only.

selected with an additional requirement of $\Delta \mathrm{R}(\mu$, jet $)>0.5$, is also included in the table.

The same method as above can be applied by requiring the second highest $p_{T}$ muon to be isolated and measure how often the leading $p_{T}$ muon is isolated as a function of the distance between the leading muon and the closest jet. The corresponding distributions of $\Delta R(\mu$, jet $)$ in data and Monte Carlo are shown in Fig. 5.7(b). It should be noted that there is an excess of events in data at small values of $\Delta R(\mu$, jet $)$ compared to the Monte Carlo. It indicates that the sample selected in this way is in fact contaminated by a background with non-isolated muons and for this reason it is not used to determine the muon isolation scale factor. 


\begin{tabular}{|l|c|c|c|}
\hline \hline & $\epsilon_{\text {data }}^{\text {event }}$ & $\epsilon_{\mathrm{MC}}^{\text {event }}$ & scale factor \\
\hline $\mathrm{N}_{\text {jet }} \geq 0$ & $0.969 \pm 0.002$ & $0.998 \pm 0.000$ & $0.971 \pm 0.002$ \\
$\mathrm{~N}_{\text {jet }} \geq 1$ & $0.966 \pm 0.005$ & $0.998 \pm 0.000$ & $0.968 \pm 0.005$ \\
$\mathrm{~N}_{\text {jet }} \geq 2$ & $0.966 \pm 0.013$ & $0.998 \pm 0.001$ & $0.967 \pm .013$ \\
\hline \hline
\end{tabular}

Table 5.7: Measured efficiencies per dimuon event for the $\chi^{2}<4$ cut (on the muon track) in $Z^{0} / \gamma^{*} \rightarrow \mu \mu$ data and Monte Carlo samples, for different jet multiplicities.

\section{Efficiency of the Track $\chi^{2}$ Cut}

A cut on the quality of the track associated with the muon is applied to suppress random matching of muons and tracks. The efficiencies for the selection criterion $\chi^{2}<4$ of the muon track, are measured for different jet multiplicities, using $Z^{0} / \gamma^{*} \rightarrow \mu \mu+$ jets data and MC samples. The per event and per muon efficiencies are presented in Tab. 5.7. The per event scale factor versus the number of jets in the event, is constant within statistical errors. Therefore, one value is used for the per muon scale factor in this analysis. This is determined for events with 0 or more jets, and the value is $0.985 \pm 0.001$ (stat) (derived using Eqn. 5.3).

\subsubsection{Jet Scale Factor}

The scale factor associated with the efficiency of identifying and reconstructing a jet, is dependent on the $E_{T}$ of the jet. It is derived by dividing the 


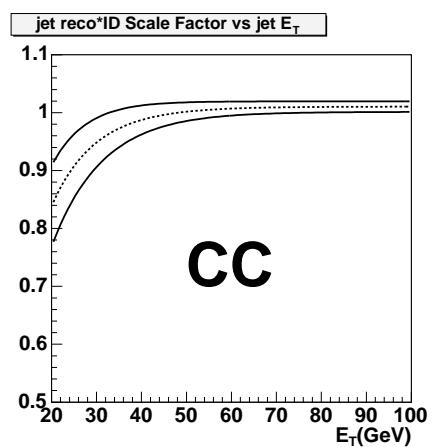

(a)

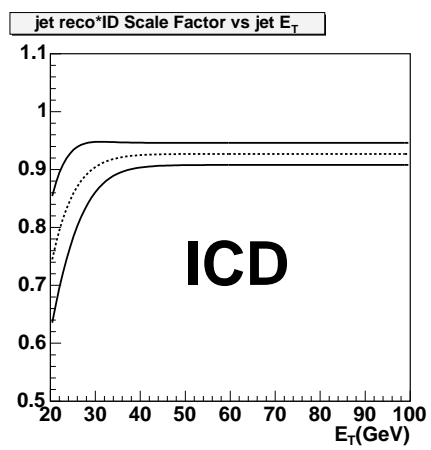

(b)

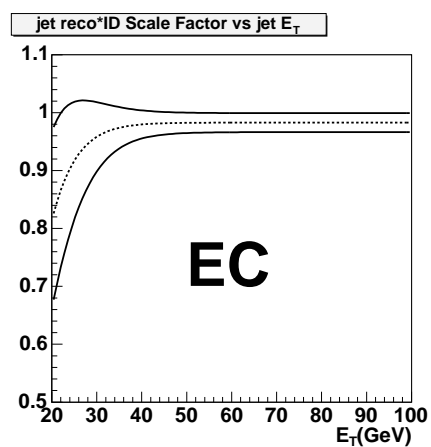

(c)

Figure 5.9: Scale factor for jet reconstruction $\times$ jet identification efficiency in $\mathrm{CC}$, ICD and EC with the associated $\pm 1 \sigma$ band used for the systematic uncertainty on the central value.

probability to reconstruct and identify a jet in data by the same probability in Monte Carlo events. Both $\gamma+$ jet and $Z^{0} / \gamma^{*}+$ jet samples have been studied to derive this scale factor. Because of the low statistics of the $Z^{0} / \gamma^{*}+$ jet dataset, the final scale factors are derived using $\gamma+$ jet data and MC samples. Parametrization of this scale factor as a function of $E_{T}$ of the jet for three different regions, viz., CC, ICD and EC is shown in Fig. 5.9. The jet reconstruction and identification efficiencies derived from signal and background Monte Carlo samples, are corrected using these $E_{T}$ dependent scale factors. 


\subsection{Signal Efficiency}

The efficiencies for various selection criteria used to identify $t \bar{t} \rightarrow e \mu$ decays where the electron and muon originate from a $W^{ \pm}$directly, or from the leptonic decay of a $\tau$-lepton which itself comes from $W^{ \pm}$decays, are quoted in Tab. 5.8. Since these efficiencies are based on an Alpgen Monte Carlo sample (see Sec. 4.3), several scale factors are required to rectify the Monte Carlo efficiencies. These scale factors include corrections due to PV selection efficiency (see Sec. 5.3.1), EM cluster reconstruction and electron identification, electron track match and electron likelihood efficiencies (see Sec. 5.3.2), muon reconstruction and identification, muon track match, muon DCA significance criterion, muon isolation and muon track $\chi^{2}$ criterion (see Sec. 5.3.3), jet reconstruction and identification (see Sec. 5.3.4). Since the scale factors are different for $\mathrm{CC}$ and EC electrons, electron scale factors from Tab. 5.4 are weighted according to the number of $\mathrm{CC}$ and $\mathrm{EC}$ electrons in the sample. The trigger efficiency for $t \bar{t} \rightarrow e \mu$ process has been obtained from Tab. 4.4 of Sec. 4.2.2. 


\begin{tabular}{|c|c|c|}
\hline$\overline{\text { Cut }}$ & Efficiency & Cumulative \\
\hline Loose electron with $p_{T}>15 \mathrm{GeV}$ & $0.6596 \pm 0.0039$ & $0.6596 \pm 0.0039$ \\
\hline$\kappa_{\text {reco+ID }}$ & 0.9672 & $0.638 \pm 0.0038$ \\
\hline Electron Likelihood+Track-matching & $0.8057 \pm 0.0041$ & $0.514 \pm 0.0040$ \\
\hline$\kappa_{\text {trk }+ \text { lhood }}$ & 0.8568 & $0.4404 \pm 0.0034$ \\
\hline Muon ID $+p_{T}>15 \mathrm{GeV}$ & $0.5942 \pm 0.0057$ & $0.2557 \pm 0.0032$ \\
\hline$\kappa_{\mu} \mathrm{ID}$ & 1.01 & $0.2582 \pm 0.0032$ \\
\hline Muon Track matching & $1.00 \pm 0.00$ & $0.2582 \pm 0.0032$ \\
\hline$\kappa_{\mu}$ Track matching & 0.985 & $0.2543 \pm 0.0031$ \\
\hline Muon Track- $\chi^{2}$ & $0.9987 \pm 0.0005$ & $0.2540 \pm 0.0031$ \\
\hline$\kappa_{\mu}$ Track $-\chi^{2}$ & 0.985 & $0.2502 \pm 0.0031$ \\
\hline Muon Isolation & $0.8378 \pm 0.0055$ & $0.2096 \pm 0.0029$ \\
\hline$\kappa_{\text {Iso }}$ & 1.00 & $0.2096 \pm 0.0029$ \\
\hline Muon DCA Significance & $0.9422 \pm 0.0038$ & $0.1975 \pm 0.0029$ \\
\hline$\kappa_{\text {DCA significance }}$ & 0.994 & $0.1963 \pm 0.0029$ \\
\hline$\Delta R(e, \mu)>0.25$ & $1.0 \pm 0.0$ & $0.1963 \pm 0.0029$ \\
\hline Trigger & $0.9377 \pm 0.0041$ & $0.1841 \pm 0.0028$ \\
\hline$>=1$ Jet with $p_{T}>20 \mathrm{GeV}$ & $0.9847 \pm 0.0021$ & $0.1813 \pm 0.0028$ \\
\hline$>=2$ Jet with $p_{T}>20 \mathrm{GeV}$ & $0.7569 \pm 0.0073$ & $0.1372 \pm 0.0025$ \\
\hline $\begin{array}{l}\left|z_{\mathrm{PV}}\right|<60 \mathrm{~cm}, N_{\mathrm{PV}}^{\mathrm{trk}} \geq 3 \text { and } \\
\Delta z(\mathrm{~d} \emptyset \text { root }, \mathrm{d} \emptyset \text { reco })<5 \mathrm{~cm}\end{array}$ & $0.9867 \pm 0.0022$ & $0.1354 \pm 0.0025$ \\
\hline$\kappa_{\mathrm{vtx}}$ & 0.997 & $0.1350 \pm 0.0025$ \\
\hline$\Delta z(e, P V)<1 \mathrm{~cm}$ and $\Delta z(\mu, P V)<1 \mathrm{~cm}$ & $0.9996 \pm 0.0004$ & $0.1349 \pm 0.0025$ \\
\hline$\kappa_{\mathrm{lv}}$ & 0.990 & $0.1336 \pm 0.0025$ \\
\hline$E_{T}>25 \mathrm{GeV}$ & $0.8987 \pm 0.0059$ & $0.1200 \pm 0.0024$ \\
\hline$\Delta \phi\left(\mu, E_{T}\right)>0.25 \mathrm{GeV}$ & $0.9415 \pm 0.0049$ & $0.1130 \pm 0.0023$ \\
\hline$H_{T}^{l}>140 \mathrm{GeV}$ & $0.9031 \pm 0.0063$ & $0.1020 \pm 0.0022$ \\
\hline
\end{tabular}

Table 5.8: Efficiencies of different selection cuts on a $t \bar{t} \rightarrow e \mu$ Monte Carlo sample and the data to Monte Carlo scale factors $(\kappa)$. The scale factors are explained in Sec. 5.3. Errors on the scale factors are not quoted here, as they are treated as systematic errors and are listed in Sec. 5.7. 


\subsection{Background Estimation}

The background processes for $t \bar{t} \rightarrow e \mu$ signal, have already been introduced in Sec. 1.4.2. The methodologies applied in this analysis to estimate the number of events arising from different background processes, have been described here in great details. Except for the instrumental backgrounds, the event yields for all other background processes are based on the samples described in Sec. 4.3 .

\subsubsection{Physics Backgrounds}

Background events arising from $Z^{0} / \gamma^{*} \rightarrow \tau \tau$ and $W^{+} W^{-} \rightarrow e \mu$ processes are estimated using corresponding Pythia and Alpgen samples described in Sec. 4.3. The Pythia samples are used to predict the number of events having $\geq 0$ or $\geq 1$ jet with jet $E_{T}>20 \mathrm{GeV}$, while Alpgen samples give the estimation for the number of events having $\geq 2$ jets for each of these two processes. Since the estimation of event yields are based on MC efficiencies, they are corrected with different scale factors, mentioned in Sec. 5.3. The event yields for $Z^{0} / \gamma^{*} \rightarrow \tau \tau$ and $W^{+} W^{-} \rightarrow e \mu$ are further corrected for individual trigger efficiencies, as obtained from Tab. 4.4 of Sec. 4.2.2. The samples are normalised to the integrated luminosity of the dataset presented 
in this analysis, with the cross sections given in Sec. 4.3.

The background contribution from the $W^{ \pm} Z^{0} \rightarrow e \mu$ decays which pass different event selection criteria, is determined using the corresponding Pythia sample, described in Sec. 4.3. The signal contamination from this particular background process is significantly small.

The estimated event yields for these three physics processes are given Tab. 5.17, at the various stages of event selection.

\subsubsection{Photon Backgrounds}

As mentioned earlier in Sec. 4.3.1, two categories of photon background processes reproduce muon-photon final states, which become apparent as muonelectron events. The photon is either produced as bremsstrahlung radiation from a high $p_{T}$ muon or it is directly produced in association with a $W^{ \pm}$ $\left(\rightarrow \mu \nu_{\mu}\right)$ or $Z^{0}(\rightarrow \mu \mu)$ boson. In both cases the photon cluster in the calorimeter gets associated to a track in the central tracking system and becomes an electron candidate.

\section{Bremsstrahlung Backgrounds}

A muon in $W^{ \pm} \rightarrow \mu \nu_{\mu}$ and $Z^{0} / \gamma^{*} \rightarrow \mu \mu$ events can radiate a bremsstrahlung photon, which is expected to be very close (in $\eta-\phi$ space) to the direction of 
the radiating muon. Such photons may result in following two possibilities. If the muon which radiates the photon is not reconstructed, the photon produces an isolated electromagnetic cluster in the calorimeter with a matched track (originally the muon track) in the central tracking system and thus becomes a perfect electron candidate. The other possibility is the simplest scenario when both muon and the photon are reconstructed. The latter can be easily rejected by applying a selection criteria based on $\Delta R(e, \mu)$ between the muon and the candidate electron (actually photon). The former case is very difficult to discriminate against real electrons and the events can be rejected by checking the $p_{T}$ mismatch between the calorimeter cluster (from photon) and the track in the central tracking system (from muon).

Fig. 5.10 shows the $\Delta R(e, \mu)$ distribution for data, and for the $W^{ \pm} \rightarrow$ $\mu \nu_{\mu}$ and $Z^{0} / \gamma^{*} \rightarrow \mu \mu$ processes (mentioned as $Z \rightarrow \mu \mu$ in the figure), in the events containing one tight isolated muon and one tight electron both having $p_{T}>15 \mathrm{GeV}$. The contribution from $Z^{0} / \gamma^{*} \rightarrow \tau \tau$ process is also displayed, as it is the most dominating background process at this level of event selection. The events from $W^{ \pm} \rightarrow \mu \nu_{\mu}$ and $Z^{0} / \gamma^{*} \rightarrow \mu \mu$ processes clearly exhibit a population of collinear pairs of electrons and muons (shown in the first bin of Fig. 5.10). $34.77 \pm 6.26$ and $\approx 7.23$ are the estimated numbers of such events arising from $W^{ \pm} \rightarrow \mu \nu_{\mu}$ and $Z^{0} / \gamma^{*} \rightarrow \mu \mu$ processes respectively, 


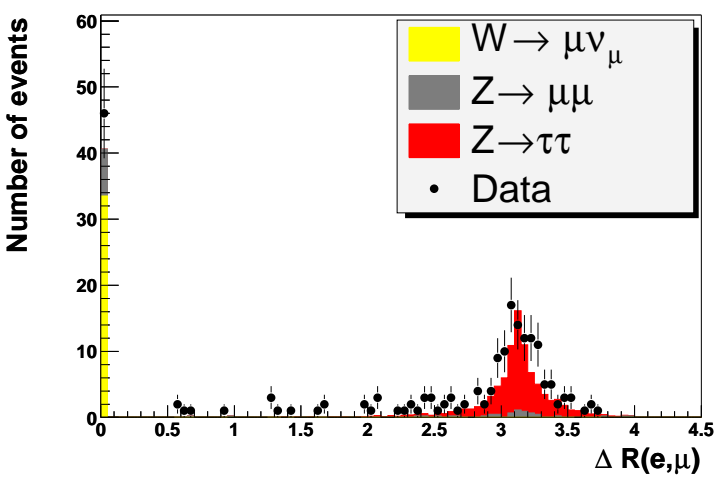

Figure 5.10: $\Delta R(e, \mu)$ distribution in data (markers), and in $Z^{0} / \gamma^{*} \rightarrow \mu \mu$, $W^{ \pm} \rightarrow \mu \nu_{\mu}$, and $Z^{0} / \gamma^{*} \rightarrow \tau \tau$ processes, where an event consists of one tight electron and one tight isolated muon. It is to be noted that the Monte Carlo prediction at the first bin i.e., $\approx 42$ events, matches quite well with the data observation of $46 \pm 6.78$ events (see Tab. 5.17).

as obtained using the MC samples described in Sec. 4.3.1. Thus a data observation of $46 \pm 6.78$ such events is consistent with that estimated from MC. Application of $\Delta R(e, \mu)>0.25$ selection criterion, rejects all $W^{ \pm} \rightarrow \mu \nu$ events, along with nearly $50 \%$ rejection for $Z^{0} / \gamma^{*} \rightarrow \mu \mu$ events.

\section{Associated Photon Production}

Another source of photon background events arises from $W^{ \pm} \gamma \rightarrow \mu \nu_{\mu} \gamma$ and $Z^{0} \gamma \rightarrow \mu \mu \gamma$ processes. Contrary to bremsstrahlung photons, this type of photons are isolated from the direction of the muon(s) in an event. Here the photon can appear as an electron candidate only if, it overlaps with a random 
track accidentally or it gets converted into an $e^{+} e^{-}$pair in the material before the calorimeter. The calorimetric measurements of these photons should resemble those of electrons while, the rate to match a central track should be considerably lower than that for real electrons. The parameters of such a candidate electron $\left(p_{T}\right.$, spatial match, track isolation, $\left.\mathrm{E}_{\mathrm{T}} / \mathrm{p}_{\mathrm{T}}\right)$ are expected to be different from those of a real electron or a jet mimicking an electron. As a result, it is highly desirable to estimate a rate at which a photon can be misidentified as an electron candidate, $f_{\gamma}$. But it is quite non-trivial to measure $f_{\gamma}$ for the events in data, because of the difficulty ${ }^{\dagger}$ to select events containing genuine isolated photons in experimental data. As an alternative, this analysis uses a value of $f_{\gamma}$, which is obtained by using MC samples. The clusters passing 'medium' electron criteria in the $W^{ \pm} \gamma \rightarrow \mu \nu_{\mu} \gamma$ and $Z^{0} \gamma \rightarrow \mu \mu \gamma$ samples (see Sec. 4.3.1) are selected first and the rate at which these clusters pass the track match and likelihood criteria gives $f_{\gamma}$. The values of $f_{\gamma}$ measured separately in the said MC samples, are presented in Tab. 5.9. The measured values are similar but not identical in the two

\footnotetext{
${ }^{\dagger}$ The most trivial sample containing $\gamma+$ jets events are selected from the experimental data, by requiring a photon cluster in the calorimeter back to back with a jet. In this case, the events are required to have the photon clusters which do not have any tracks in the vicinity of the clusters and thus possible contamination from electrons or $\pi^{0}$ 's (inside a jet) is reduced to a great extent. Thus the said sample is not usable for the current purpose of measuring $f_{\gamma}$ in data.
} 
samples, so an average between them is assumed to represent $f_{\gamma}$ in Monte Carlo events.

\begin{tabular}{|c||c|c|c|}
\hline \hline Criteria & $W^{ \pm} \gamma$ & $Z^{0} \gamma$ & Average \\
\hline Track match & $7.21 \pm 0.41 \%$ & $9.41 \pm 0.65 \%$ & $8.31 \pm 0.38 \%$ \\
$f_{\gamma}$ (Track match+Likelihood) & $1.66 \pm 0.20 \%$ & $2.03 \pm 0.31 \%$ & $1.84 \pm 0.18 \%$ \\
\hline
\end{tabular}

Table 5.9: The rate for photon faking an electron candidate, $f_{\gamma}$, is estimated from Monte Carlo samples, as a probability of isolated photon cluster to pass track match and likelihood criteria. The rate at which photon clusters pass track match criteria is also provided separately. The average is taken over the rates from two samples (viz., $W^{ \pm} \gamma \rightarrow \mu \nu_{\mu} \gamma$ and $Z^{0} \gamma \rightarrow \mu \mu \gamma$ ).

The measurement of $f_{\gamma}$ from MC samples following above procedure, might be an underestimate for the same rate in data, due to the following reason. $f_{\gamma}$ is dominated by the conversion of a photon into a $e^{+} e^{-}$pair in the central tracking system when one of the tracks either from electron or from positron, matches with the combined cluster $^{\S}$, formed in the calorimeter. Thus $f_{\gamma}$ is affected by the amount of material in the central tracking system which is not modelled properly in current version of dØgstar. The mismatch between the SMT geometry in dØgstar and that in the actual actual detector, amounts to a discrepancy of $20 \%$, in terms of $\gamma \rightarrow e^{+} e^{-}$

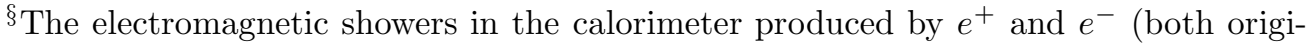
nating from a photon conversion), can not be separated out from each other, thus appear as a combined cluster having roughly the energy of the photon. Using the information from CPS or FPS, $e^{+} e^{-}$clusters can be resolved, but the data for present analysis does not have these information.
} 
conversion rate [92] and is taken as the systematic uncertainty on the photon background estimation of this analysis.

\section{Event Yield}

As described above, the event yields for different photon background processes are based on Monte Carlo event simulation. These MC based event yields are rectified for the trigger efficiencies (see Tab. 4.4) and different data to $\mathrm{MC}$ scale factors (see Sec. 5.3). In the case of the associated production processes, events are selected first with one medium candidate electron and a tight isolated muon. The events are then weighted with average $f_{\gamma}$, quoted in Tab. 5.9. The $\Delta R(e, \mu)>0.25$ selection criteria removes majority of the bremsstrahlung background processes leaving approximately $2 / 3$ of the photon background processes which come from associated production processes, $W^{ \pm} \gamma \rightarrow \mu \nu_{\mu} \gamma$ process in particular. After applying all selection criteria, this background process contributes $0.02 \pm 0.01$ events. Tab. 5.5.2 indicates the expectation for each of the four photon background processes.

\subsubsection{Instrumental Fakes}

As introduced in Sec. 1.4.2, instrumental backgrounds contributing to this analysis contain misidentified leptons, due to the detectors effects. These 


\begin{tabular}{|c||c|c|c|c|}
\hline \hline Cut & $Z^{0} / \gamma^{*} \rightarrow \mu \mu$ & $W^{ \pm} \gamma$ & $Z^{0} \gamma$ & $W^{ \pm} \rightarrow \mu \nu_{\mu}$ \\
\hline $1 e$ and $1 \mu$ & $14.77 \pm 2.17$ & $11.97 \pm 1.24$ & $7.50 \pm 0.81$ & $34.77 \pm 6.26$ \\
\hline$\Delta R(e, \mu)>0.25$ & $7.54 \pm 1.44$ & $11.69 \pm 1.22$ & $7.32 \pm 0.79$ & - \\
\hline$\geq 1$ jet & $0.90 \pm 0.45$ & $1.49 \pm 0.19$ & $1.06 \pm 0.14$ & - \\
\hline$\geq 2$ jets & $0.22 \pm 0.22$ & $0.11 \pm 0.03$ & $0.07 \pm 0.02$ & - \\
\hline$\not_{T}>25 \mathrm{GeV}$ & - & $0.08 \pm 0.03$ & $0.03 \pm 0.01$ & - \\
\hline$\Delta \phi\left(\mu, E_{T}\right)>0.25$ & - & $0.08 \pm 0.03$ & $0.03 \pm 0.01$ & - \\
\hline$H_{T}^{l}>140 \mathrm{GeV}$ & - & $0.02 \pm 0.01$ & - & - \\
\hline
\end{tabular}

Table 5.10: Expected event yields for various photon background processes. All errors are statistical.

events can appear from following three different background processes

- QCD multijet production where both electron and muon are misidentified,

- $W^{ \pm}\left(\rightarrow \mu \nu_{\mu}\right)+$ jets decays where the candidate electron is mimicked by the calorimetric fluctuation of a hadronic shower from a jet,

- $W^{ \pm}\left(\rightarrow e \nu_{e}\right)+\mathrm{b} \overline{\mathrm{b}}$ events where a isolated muon appears from the semileptonic decay(s) of b quark(s).

Provided that the probabilities for jets to fake electrons, $f_{e}$, is not correlated in the QCD sample with the probability for a $\mu$ from a semileptonic decay (of b quark) to appear isolated, $f_{\mu}$, the number of such misidentified events in the experimental data having one electron and one muon, can be written 
as,

$$
N^{\text {Fake }}=\left(N^{Q C D} \cdot f_{\mu}+N^{W^{ \pm}\left(\rightarrow \mu \nu_{\mu}\right)+j e t s}\right) \cdot f_{e}+N^{W^{ \pm}\left(\rightarrow e \nu_{e}\right)+b \bar{b}} \cdot f_{\mu}
$$

where $\mathrm{N}^{\mathrm{QCD}}, \mathrm{N}^{ \pm}\left(\rightarrow \mu \nu_{\mu}\right)+$ jets and $\mathrm{N}^{ \pm}\left(\rightarrow \mathrm{e} \nu_{\mathrm{e}}\right)+\mathrm{b} \overline{\mathrm{b}}$, are the numbers of QCD, $W^{ \pm}\left(\rightarrow \mu \nu_{\mu}\right)+$ jets and $W^{ \pm}\left(\rightarrow e \nu_{e}\right)+b \bar{b}$ events respectively, in the data sample under consideration.

\section{Fake Electron}

In order to estimate the number of events in experimental data where the candidate electrons are faked by fluctuation of hadronic showers in the calorimeter, the contribution from $W^{ \pm}\left(\rightarrow e \nu_{e}\right)+b \bar{b}$ events are neglected ${ }^{\dagger}$ Thus Eqn. 5.4 can be rewritten as,

$$
N^{\text {Fake }}=\left(N^{Q C D} \cdot f_{\mu}+N^{W^{ \pm}\left(\rightarrow \mu \nu_{\mu}\right)+j e t s}\right) \cdot f_{e}
$$

The quantity inside the parentheses is the number of events containing isolated muons which can be directly taken from the experimental data. Thus one needs to measure $f_{e}$ only.

\footnotetext{
$\dagger$ Neglecting such contribution does not change the results under study, as shown later in the context of four-component Matrix method.
} 
The electron fake rate is determined using a sample containing a loose electron and a medium muon both with $p_{T}>15 \mathrm{GeV}$. This sample is primarily composed of QCD events in which the loose electrons are mostly jets and the medium muons are mostly the non-isolated muons from jets. The electron candidates in these events are then required to pass the 'tight' electron selection criteria (track match and likelihood). Fig. 5.11(a,b) show the two dimensional distributions of 'Rattrk' vs. 'Rat11' of the muons (see Sec. 3.2.3 for the definitions of 'Rattrk' and 'Rat11' variables) in these two sets of events with CC and EC electrons, respectively. As evident from the graph, in the events having tight electrons, both parameters ('Rattrk' and 'Rat11') are correlated at low values, where the physics processes consisting of a real electron and a real isolated muon such as, $Z^{0} / \gamma^{*} \rightarrow \tau \tau \rightarrow e \mu$, $W^{+} W^{-} \rightarrow e \mu$ or $t \bar{t} \rightarrow e \mu$ events, are dominant. In order to remove these events from the selected dataset having a loose electron and a medium muon and to enrich the sample with QCD events for the purpose of measuring $f_{e}$, the events with isolated muons (in this sample) are rejected. $f_{e}$ is obtained using this sample, as the ratio of the number of events with tight electrons to that with loose electrons for the events where the muons satisfy the requirement, "Rat11>0.12 AND Rattrk> 0.12". Altering the rejection criteria for the isolated muons in the sample to "Rat11>0.2 AND Rattrk $>0.2$ " does 


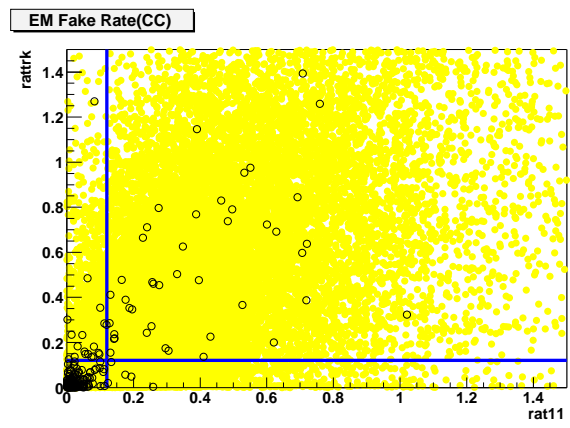

(a)

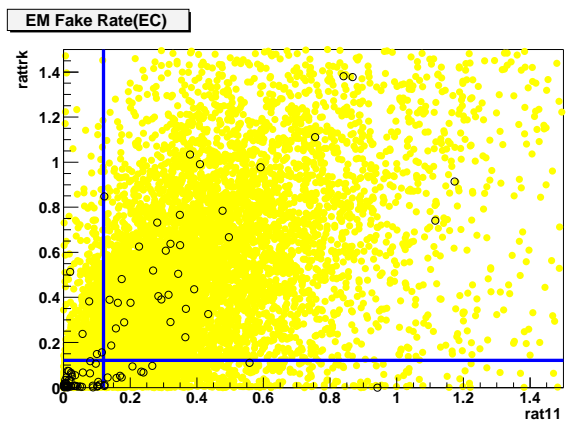

(b)

Figure 5.11: Correlation of 'Rattrk' and 'Rat11' parameters of the muon, in the events with 'loose' and 'tight' electrons in (a) CC and (b) EC. The events containing one 'medium' muon and one 'loose' ('tight') electron are represented by the points with yellow (black) marker. The region at bottom left corner of each plot contains physics backgrounds. These events are removed by only accepting events with non-isolated muons in upper right quadrant of each plot.

not affect the measured value of $f_{e}$. This provides the confidence that the measured value of $f_{e}$ is not biased by possible contamination from physics processes containing real electrons. The electron fake rate is measured separately for $\mathrm{CC}$ and $\mathrm{EC}$. In the above sample, the number of events containing one loose electron, and one tight isolated muon i.e., with "Rat11<0.12 AND Rattrk $<0.12$ " is determined first. The estimate for the events containing fake electrons is obtained by multiplying this number of events, with measured $f_{e}$. Tab. 5.17 shows the numbers of such background events at different levels of event selection. Tab. 5.11 shows values of $f_{e}$ for $\mathrm{CC}$ and EC elec- 
trons, and $12.45 \pm 1.4$ (stat) is the number of such fake electron events at a level of event selection, which corresponds to the events having one tight electron and one tight isolated muon with $\Delta R(e, \mu)>0.25$.

\begin{tabular}{|c|c|c|c|}
\hline & Isolated muons & EM fake rate & Misidentified Events \\
\hline \hline CC EM & 2430 & $0.31 \pm 0.05 \%$ & $7.47 \pm 1.11$ \\
EC EM & 876 & $0.57 \pm 0.09 \%$ & $4.98 \pm 0.85$ \\
\hline \hline \multicolumn{2}{|c|}{ Total Number of fake events } & $12.45 \pm 1.4$ \\
\hline
\end{tabular}

Table 5.11: Estimated $f_{e}$ and event yield for fake electrons corresponding to an event selection criteria of having one tight electron and one tight muon with $\Delta R(e, \mu)>0.25$ (see Tab. 5.1) in the event.

\section{Fake Muon}

While estimating for the event count from fake electron background processes, two simplifications have been made, whose validity would be useful to test. First, the contribution from $W^{ \pm}\left(\rightarrow e \nu_{e}\right)+b \bar{b}$ process is neglected. Secondly, the effect of the events having one real electron and one real muon, has not been taken into consideration while modelling Eqn. 5.4. Therefore, a more general list of the processes which can result in the event signature of having one loose electron and one loose $^{\S}$ muon would be: QCD multijet, $W^{ \pm}\left(\rightarrow \mu \nu_{\mu}\right)+$ jets, $W^{ \pm}\left(\rightarrow e \nu_{e}\right)+b \bar{b}$, and $t \bar{t}$ plus physics background processes,

\footnotetext{
${ }^{\S}$ Here the 'loose' definition of a muon is different from the standard definitions (see Sec. 3.2.3), which are used frequently in this analysis.
} 
and can be described through the following equation.

$$
N^{l l}=N^{\mathrm{QCD}}+N^{W^{ \pm}\left(\rightarrow \mu \nu_{\mu}\right)+\text { jets }}+N^{W^{ \pm}\left(\rightarrow e \nu_{e}\right)+\mathrm{b} \overline{\mathrm{b}}}+N^{\text {Physics }}
$$

where $N^{\text {Physics }}$ is the contribution from all physics processes except the events containing either fake electron, or fake muon, or both. $N^{x y}$ indicates by $x$ whether muons are 'loose' or 'tight', and by $y$ whether electrons are 'loose' or 'tight'. In order to have convenient methodology and to create statistically powerful event samples, the 'loose' definition of muon has been modified, while 'tight' muons remain identical to those defined in Sec. 3.2.3. It should be noted that this 'loose' definition of muon is only valid for this section and the explicit criteria for such a definition are listed in Tab. 5.12. For clarity, 'loose' electron definition is also mentioned the table. Applying different electron and muon identification criteria i.e., loose or tight, the data sample at different levels of selection viz., $N^{l l}, N^{l t}, N^{t l}$ and $N^{t t}$ can be described by the following Matrix,

$$
\left(\begin{array}{c}
N^{l l} \\
N^{l t} \\
N^{t l} \\
N^{t t}
\end{array}\right)=\left(\begin{array}{cccc}
1 . & 1 . & 1 . & 1 . \\
f_{e} & f_{e} & \epsilon_{e}^{s i g} & \epsilon_{e}^{s i g} \\
f_{\mu} & \epsilon_{\mu}^{s i g} & f_{\mu} & \epsilon_{\mu}^{s i g} \\
f_{\mu} \cdot f_{e} & \epsilon_{\mu}^{s i g} \cdot f_{e} & f_{\mu} \cdot \epsilon_{e}^{s i g} & \epsilon_{\mu}^{s i g} \cdot \epsilon_{e}^{s i g}
\end{array}\right) \cdot\left(\begin{array}{c}
N^{Q C D} \\
N^{W\left(\rightarrow \mu \nu_{\mu}\right) j e t s} \\
N^{W\left(\rightarrow e \nu_{e}\right) b \bar{b}} \\
N^{\text {Physics }}
\end{array}\right)
$$


Here, $f_{\mu}$ is the probability for a loose muon to appear as a tight muon and is different from the $f_{\mu}$ in Eqn. 5.4 of previous section, while $f_{e}$ is the identical to the electron fake rate discussed in the last section. $\epsilon_{\mu}^{\mathrm{sig}}$ and $\epsilon_{e}^{\mathrm{sig}}$ are the efficiencies for real muons and real electrons to pass the corresponding tight criteria, respectively. Therefore, if the values for $f_{\mu}, f_{e}, \epsilon_{\mu}^{\mathrm{sig}}$ and $\epsilon_{e}^{\mathrm{sig}}$ are known, one can extract the number of events corresponding to a particular process, $N^{Q C D}, N^{W^{ \pm}\left(\rightarrow \mu \nu_{\mu}\right) j e t s}, N^{W^{ \pm}\left(\rightarrow e \nu_{e}\right) b \bar{b}}$ or $N^{\text {Physics }}$, contained in the experimental dataset by solving the matrix of Eqn. 5.7. This procedure is referred to as the four-component matrix method. Unfortunately, except the $f_{e}$, the fake electron probability as measured in the previous section, neither of the $\epsilon$ 's nor $f_{\mu}$ can be derived from the data sample containing one electron and one muon, due to the large correlation between the different processes, and due to lack of statistics.

\begin{tabular}{|c||c|c|}
\hline & Loose & Tight \\
\hline \hline Electron & $\begin{array}{c}\text { reconstructed } \\
\text { EM cluster from calorimeter }\end{array}$ & $\begin{array}{c}\text { track matched EM cluster } \\
\text { passing Likelihood criteria }\end{array}$ \\
\hline Muon & rattrk $<0.25$ AND rat11 $<0.25$ & rattrk $<0.12$ AND rat11 $<0.12$ \\
\hline
\end{tabular}

Table 5.12: Various EM and muon definitions used in Matrix method.

In order to choose an appropriate definition of 'loose' muon to be applicable to four-component Matrix method, it is to be ensured that the jet- 
multiplicities in the events containing loose muons should resemble those containing tight muons. If the isolation criteria based on rattrk and rat11 parameters of muon, are completely omitted for the 'loose' muon definition, the jet-multiplicities in the events becomes quite different from those containing 'tight' muons. With a moderate definition of 'loose' muon i.e., with "rattrk $<0.25$ AND rat11< 0.25 ", the jet-multiplicities in loose and tight muon events, remain almost identical in a data sample containing $W^{ \pm} \rightarrow \mu \nu_{\mu}+$ jets and QCD events where, $f_{\mu}$ is a function of $E_{T}$ (see Fig. 5.12). The events selected with $\mathbb{E}_{\mathrm{T}}<10 \mathrm{GeV}$ in this sample, are dominated by QCD events, where the muons comes from heavy flavour decays. In this region $\left(\mathrm{E}_{\mathrm{T}}<10\right)$, an average rate at which a loose muon appears as tight is considered to be the value of $f_{\mu}$. The $\epsilon_{\mu}^{\mathrm{sig}}\left(\epsilon_{e}^{\mathrm{sig}}\right)$ is measured from a data sample containing $Z^{0} / \gamma^{*} \rightarrow \mu \mu\left(Z^{0} / \gamma^{*} \rightarrow e e\right)$ events with $80<M_{\mu \mu}<100$ $\left(80<M_{e e}<100\right) \mathrm{GeV}$. To apply into the four-component matrix methodology, the value of $f_{e}$ is taken to be the electron fake probability, as measured in the previous section describing the fake electron background. The efficiencies and fake rates with these definitions are given in Tab. 5.13.

The breakdown for the dataset with the four different configurations of loose and tight electrons and muons is given in Tab. 5.14. The composition of the loose-loose $\left(N^{l l}\right)$ and the tight-tight $\left(N^{t t}\right)$ data samples are shown in 


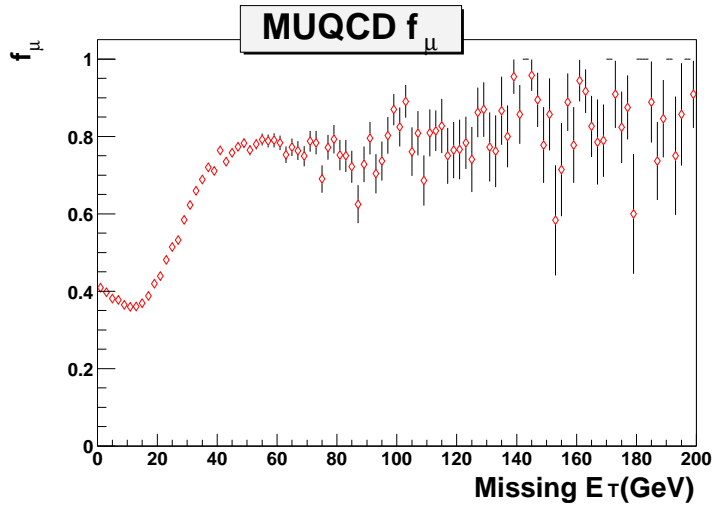

Figure 5.12: $f_{\mu}$ as a function of $\mathbb{E}_{T}$, measured in dataset containing $W^{ \pm} \rightarrow$ $\mu \nu_{\mu}+$ jets and QCD events.

\begin{tabular}{|c|c|c|}
\hline \hline & $\epsilon^{\text {sig }}$ & $f_{e, \mu}$ \\
\hline$\mu$ & $0.9383 \pm 0.002$ & $0.3819 \pm 0.0027$ \\
\hline$e$ & $0.6899 \pm 0.0032$ & $0.0038 \pm 0.0004$ \\
\hline \hline
\end{tabular}

Table 5.13: Signal and fake efficiencies for electrons and muons applied in four-component Matrix method.

Tab. 5.15, by applying the 4-component matrix method.

As shown in the second column of Tab. 5.15, the experimental dataset selected to have events containing one tight electron and one tight muon with $\Delta R(e, \mu)>0.25$, contains similar number of events from QCD, $W^{ \pm}(\rightarrow$ $\left.\mu \nu_{\mu}\right)+j e t s$ and $W^{ \pm}\left(\rightarrow e \nu_{e}\right)+b \bar{b}$ processes. Because of the low rejection power of $f_{\mu}$, event estimate for $W^{ \pm}\left(\rightarrow e \nu_{e}\right)+b \bar{b}$ process has a large statistical uncertainty. The matrix method estimates a sum of $\approx 11.77$ events from the 


\begin{tabular}{|l|c|c|}
\hline \hline & Loose EM & Tight EM \\
\hline Loose $\mu$ & 6040 & 185 \\
\hline Tight $\mu$ & 3306 & 156 \\
\hline \hline
\end{tabular}

Table 5.14: Events observed for different loose and tight configurations for electrons and muons with $\Delta R(e, \mu)>0.25$.

\begin{tabular}{|c||c|c|}
\hline \hline Components & Composition of loose-loose & Yield for tight-tight \\
\hline QCD & $4221.4 \pm 91$ & $6.13 \pm 0.70$ \\
\hline$W^{ \pm}(\rightarrow \mu \nu)+$ jets & $1582.4 \pm 76.1$ & $5.64 \pm 0.69$ \\
\hline$W^{ \pm}(\rightarrow e \nu)+$ jets & $22.56 \pm 13.57$ & $5.94 \pm 3.58$ \\
\hline Physics Processes & $213.61 \pm 20.87$ & $138.29 \pm 13.53$ \\
\hline \hline
\end{tabular}

Table 5.15: Number of events determined in loose-loose and tight-tight data samples with $\Delta R(e, \mu)>0.25$.

two fake electron backgrounds viz., QCD and $W^{ \pm}(\rightarrow \mu \nu)+$ jets processes, which is in agreement with the value of $12.45 \pm 1.4$ events, estimated applying the methodology described in the previous section. The four component matrix method described here, runs into fatal statistical limitations when applied on the events having $\geq 1$ jet. As a result, the method discussed in the previous section is used to estimate total fake electron background at different levels of event selection criteria. The agreement between the two estimates for this background at the same level of event selection, justifies this approach. To estimate for the events containing fake muons from $W^{ \pm}\left(\rightarrow e \nu_{e}\right)+b \bar{b}$ process at subsequent levels of event selection, relative kinematic efficiencies 
with respect to the level at which this background is estimated using Matrix method, is derived first using a $W^{ \pm} \rightarrow e \nu_{e}$ Pythia Monte Carlo sample. The estimate for the events containing fake muons at different levels of event selection, is then obtained by multiplying the number of events (from Matrix method) with the relative kinematic efficiencies (from $W^{ \pm} \rightarrow e \nu_{e} \mathrm{MC}$ ).

It should be noted that the four-component Matrix method, does not account for the presence of the events containing photons, described in Sec. 5.5.2 because of the following reason. First the photon background processes have negligible contribution after applying the final event selection criteria. Hence they are of secondary importance compared to those considered here. Additionally, the large uncertainty in the photon fake rate implies that, it is difficult to extract accurate estimates for the number of events from different background processes. However, with the nominal $f_{\gamma}$ based on $\mathrm{MC}$ events (see Sec. 5.5.2), give results which are consistent with those shown in Tab. 5.15.

\section{Complementary Instrumental Background Estimation}

A complementary estimate for the number of misidentified events in the data sample under consideration is done by comparing the number of events with like-charged and unlike-charged leptons. In misidentified events, the 
charges of the leptons are expected to be uncorrelated. In other words, if physics processes are defined as those having oppositely charged leptons and the misidentified background events possess no charge correlations, then the sum of physics background events, $N^{\text {Physics }}$, and misidentified background events, $N^{\text {misID }}$, is given by

$$
N^{\text {Physics }}+N^{\text {misID }}=N^{\mathrm{LS}}+N^{\mathrm{ULS}},
$$

where $N^{\mathrm{LS}}$ and $N^{\mathrm{ULS}}$ are the number of events with like-signed and unlikesigned $e \mu$ lepton pairs. The events containing bremsstrahlung photons from $Z^{0} / \gamma^{*} \rightarrow \mu \mu$ decays should contribute to $N^{\text {Physics }}$ since the lepton charges will be opposite in this type of events. On the other hand, the associated photon production processes $\left(W^{ \pm} \gamma \rightarrow \mu \nu_{\mu} \gamma, Z^{0} \gamma \rightarrow \mu \mu \gamma\right)$ should be grouped with $N^{\text {misID }}$. It is expected that half of the misidentified events have likesigned lepton pairs while the remaining half should contain leptons of opposite signs,

$$
\begin{aligned}
N^{\mathrm{LS}} & =\frac{1}{2} N^{\mathrm{misID}} \\
N^{\mathrm{ULS}} & =N^{\text {Physics }}+\frac{1}{2} N^{\mathrm{misID}}
\end{aligned}
$$


Therefore, $N^{\text {misID }}=2 * N^{\mathrm{LS}}$. The numbers of unlike-charged events in the experimental dataset is 65 and 79 for $e^{+} \mu^{-}$and $e^{-} \mu^{+}$, respectively. The numbers of like-charged events are 5 and 7 for $e^{+} \mu^{+}$and $e^{-} \mu^{-}$, respectively. These events are selected from the dataset by requiring one electron and one muon with $p_{T}>15 \mathrm{GeV}$ and $\Delta R(e, \mu)>0.25$. Therefore, the number of misidentified events in this sample is

$$
N^{\text {misID }}=24 \pm 2 * \sqrt{12}=24 \pm 6.93 .
$$

Tab. 5.16 summarises the results consisting of the estimates for photon and fake background events, obtained using different methodologies. By summing the first three rows of the table, one sees that the alternate estimation provides for a comparable level of background as estimated using other methods. Nevertheless the like-sign lepton method gives an estimate for misidentified events which is $39 \%$ lower than the sum of fake and photon background events, estimated with the other techniques. This analysis considers such a discrepancy as the systematic uncertainty on the number of fake background events. 


\begin{tabular}{|c|c|}
\hline Background & Contribution \\
\hline Photon & $\overline{18.82 \pm 1.45^{a}}$ \\
\hline Fake electron & $12.45 \pm 1.4$ \\
\hline Fake isolated muon & $5.94 \pm 3.58$ \\
\hline Total & $39.46 \pm 4.29$ \\
\hline \multicolumn{2}{|c|}{ Alternate method } \\
\hline Total & $24 \pm 6.93$ \\
\hline
\end{tabular}

Table 5.16: Comparison between different methods for photon and fake background estimation. The events required to have one electron and one muon with $\Delta R(e, \mu)>0.25$. The errors are purely statistical.

${ }^{a}$ This is the contribution from $W^{ \pm} \gamma \rightarrow \mu \nu_{\mu} \gamma$ and $Z^{0} \gamma \rightarrow \mu \mu \gamma$ from Tab. 5.5.2.

\subsection{Final Event Yields}

The expected number of background and signal events in comparison to data at different levels of event selection is shown in Tab. 5.17. Expected number of signal events is computed assuming a $t \bar{t}$ production cross section of 7 pb and a branching fraction of 0.0316 (which includes the decays involving $\tau \rightarrow e / \mu)$. A summary of observed and expected background yields with statistical and systematic errors after applying the final selection criteria is presented in Tab. 5.18.

Figs. 5.13 - 5.17 show various kinematic distributions for the events containing one electron and a muon with $\Delta R(e, \mu)>0.25$ (second row of Tab. 5.17). Though this analysis does not use any selection criteria based on the aplanarity and sphericity of the events, but the corresponding distribu- 


\begin{tabular}{|c|c|c|c|c|c|c|c|c|}
\hline \multirow[t]{3}{*}{ Selection Criteria } & \multirow[t]{3}{*}{ Data } & \multirow{3}{*}{$\begin{array}{c}\text { Total } \\
\text { (Signal+ } \\
\text { background) }\end{array}$} & \multicolumn{5}{|c|}{ Background } & \multirow{3}{*}{$\begin{array}{c}\text { Signal } \\
(t \bar{t})\end{array}$} \\
\hline & & & \multicolumn{2}{|c|}{ Fakes } & \multirow[t]{2}{*}{$Z^{0} / \gamma^{*} \rightarrow \tau \tau$} & \multirow[t]{2}{*}{$W^{+} W^{-}, W^{ \pm} Z^{0}$} & \multirow[t]{2}{*}{$\gamma$-processes } & \\
\hline & & & $\mathrm{QCD}, W \rightarrow \mu \nu_{\mu}$ & $W \rightarrow e \nu_{e}$ & & & & \\
\hline $1 e$ and $1 \mu$ & 202 & $202.6_{-13.8}^{+13.7}$ & $14.63 \pm 5.24$ & $\overline{5.94 \pm 4.11}$ & $87.46_{-7.29}^{+6.96}$ & $177.21_{-6.02}^{+6.00}$ & $\begin{array}{l}68.33_{-7.57}^{+7.56} \\
\end{array}$ & $9.01_{-0.56}^{+0.51}$ \\
\hline$\Delta R(e, \mu)>0.25$ & 156 & $158.3_{-12.0}^{+11.8}$ & $12.45 \pm 4.46$ & $5.94 \pm 4.11$ & $87.4_{-7.4}^{+7.0}$ & $17.2 \pm 6.0$ & $26.28_{-3.83}^{+3.81}$ & $9.01_{-0.56}^{+0.51}$ \\
\hline$\geq 1$ jet & 30 & $29.4_{-2.8}^{+3.6}$ & $2.89 \pm 1.04$ & $0.74 \pm 0.51$ & $11.97_{-2.22}^{+3.21}$ & $1.55 \pm 0.64$ & $3.42 \pm 0.85$ & $8.88_{-0.55}^{+0.52}$ \\
\hline$\geq 2$ jets & 10 & $10.1 \pm 0.8$ & $0.49 \pm 0.17$ & $0.08 \pm 0.05$ & $1.73_{-0.43}^{+0.42}$ & $0.70_{-0.27}^{+0.30}$ & $0.39_{-0.32}^{+0.23}$ & $6.72_{-0.52}^{+0.53}$ \\
\hline$E_{T}>25 \mathrm{GeV}$ & 10 & $8.18_{-0.59}^{+0.60}$ & $0.34 \pm 0.12$ & $0.06 \pm 0.04$ & $1.00 \pm 0.22$ & $0.60_{-0.24}^{+0.25}$ & $0.11_{-0.07}^{+0.006}$ & $6.07_{-0.46}^{+0.48}$ \\
\hline$\Delta \phi\left(\mu, E_{T}\right)>0.25$ & 8 & $7.59_{-0.54}^{+0.60}$ & $0.33 \pm 0.12$ & $0.06 \pm 0.04$ & $0.83_{-0.20}^{+0.18}$ & $0.56_{-0.23}^{+0.24}$ & $0.11_{-0.07}^{+0.06}$ & $5.70_{-0.42}^{+0.49}$ \\
\hline$H_{T}^{l}>140 \mathrm{GeV}$ & 8 & $6.05_{-0.51}^{+0.60}$ & $0.17 \pm 0.06$ & $0.02 \pm 0.01$ & $0.35_{-0.09}^{+0.10}$ & $0.35_{-0.14}^{+0.17}$ & $0.02_{-0.03}^{+0.02}$ & $5.14_{-0.48}^{+0.58}$ \\
\hline
\end{tabular}

Table 5.17: Data, expected signal and backgrounds at each level of selection in electron-muon channel, with statistical errors added quadratically to the systematic uncertainties. The expected signal yield is based on a $t \bar{t}$ production cross section of $7 \mathrm{pb}$. 


\begin{tabular}{|r|c|c|c|}
\hline \hline Category & Yield & Stat Err & Sys Err \\
\hline$Z / \gamma^{*} \rightarrow \tau \tau$ & 0.35 & \pm 0.06 & \pm 0.07 \\
$W W, W Z$ & 0.35 & \pm 0.00 & $+0.17-0.14$ \\
$\gamma$-processes & 0.02 & \pm 0.02 & $+0.01-0.02$ \\
Fakes (QCD, $W \rightarrow e \nu, W \rightarrow \mu \nu)$ & 0.19 & \pm 0.02 & \pm 0.06 \\
\hline Total background & 0.91 & \pm 0.07 & $+0.20-0.17$ \\
\hline Expected Signal & 5.14 & \pm 0.11 & $+0.57-0.47$ \\
\hline Selected Events & 8 & & \\
\hline \hline
\end{tabular}

Table 5.18: Expected background and observed and expected signal yields. The expected signal yield assumes a $7 \mathrm{pb} t \bar{t}$ production cross section.

tions are shown in Figs. 5.18 and 5.19 respectively, for completeness. Similar plots are shown in Figs.5.20 - 5.24 for the events which are required to have $\geq 1$ jet. Figs. 5.25- 5.30 show distributions of $p_{T}$ and $\eta$ of electron, leading lepton $p_{T}, p_{T}$ and $\eta$ of muon, $p_{T}$ and $\eta$ of leading jet, $E_{T}, H_{T}^{\text {leading lepton }}, H_{T}^{\text {jets }}$, number of jets, for signal and various background processes. Eight events in the data pass all of the selection cuts. The run numbers and event numbers of these events are presented in the Tab. 5.19. The kinematics of these events are given in Tabs. C.1- C.8 with the graphical displays in Figs. C.1- C.8 in Appendix C. 

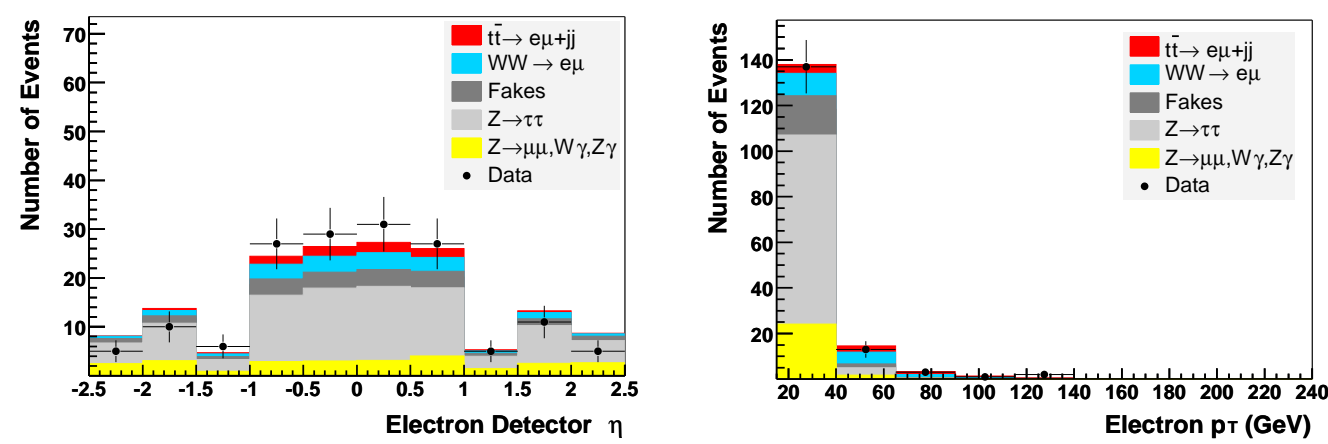

Figure 5.13: Distributions for detector $\eta$ (left) and $p_{T}$ (right) of electrons in $t \bar{t} \rightarrow e \mu$, background and data events having one good electron and one good muon with $\Delta R(e, \mu)>0.25$.
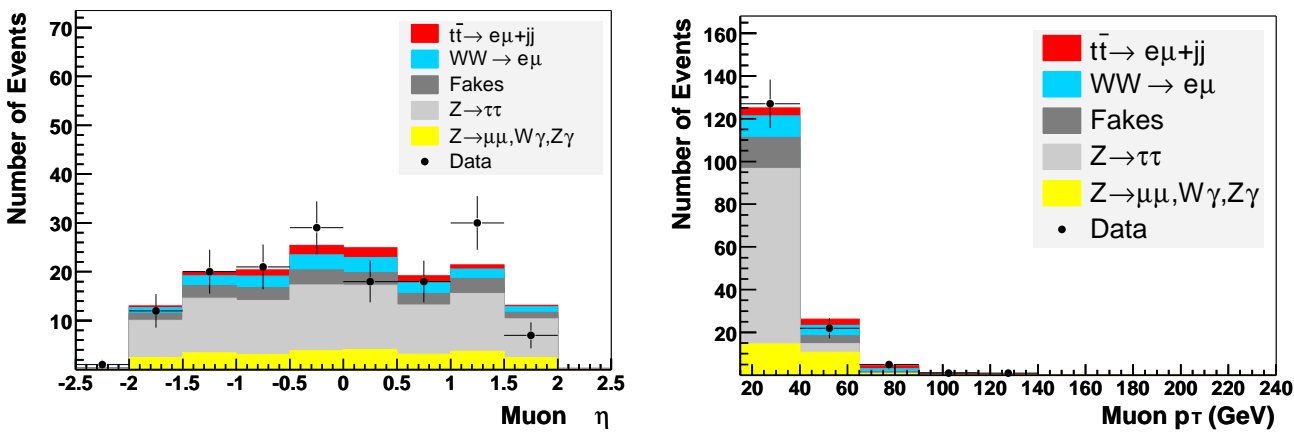

Figure 5.14: Distributions for $\eta$ (left) and $p_{T}$ (right) of muons in $t \bar{t} \rightarrow e \mu$, background and data events having one good electron and one good muon with $\Delta R(e, \mu)>0.25$. 


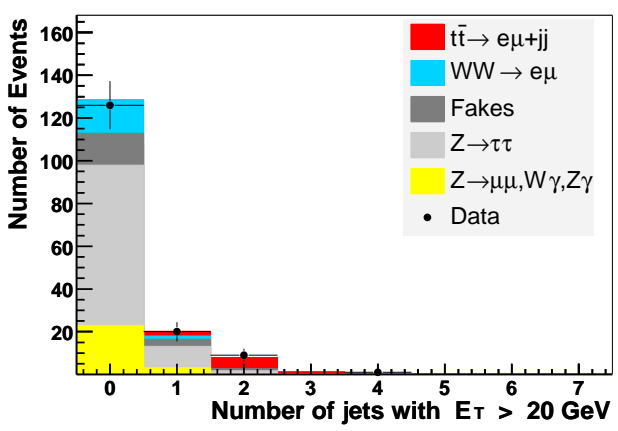

(a)

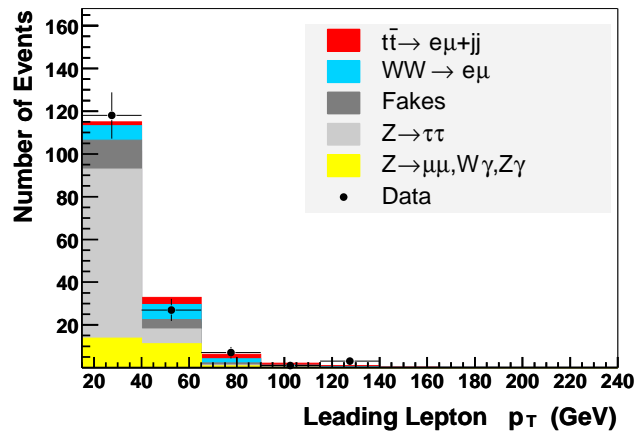

(b)

Figure 5.15: Distributions of (a) number of jets having $p_{T}>20 \mathrm{GeV}$ and (b) $p_{T}$ of the leading lepton, in $t \bar{t} \rightarrow e \mu$, background and data events having one good electron and one good muon with $\Delta R(e, \mu)>0.25$.
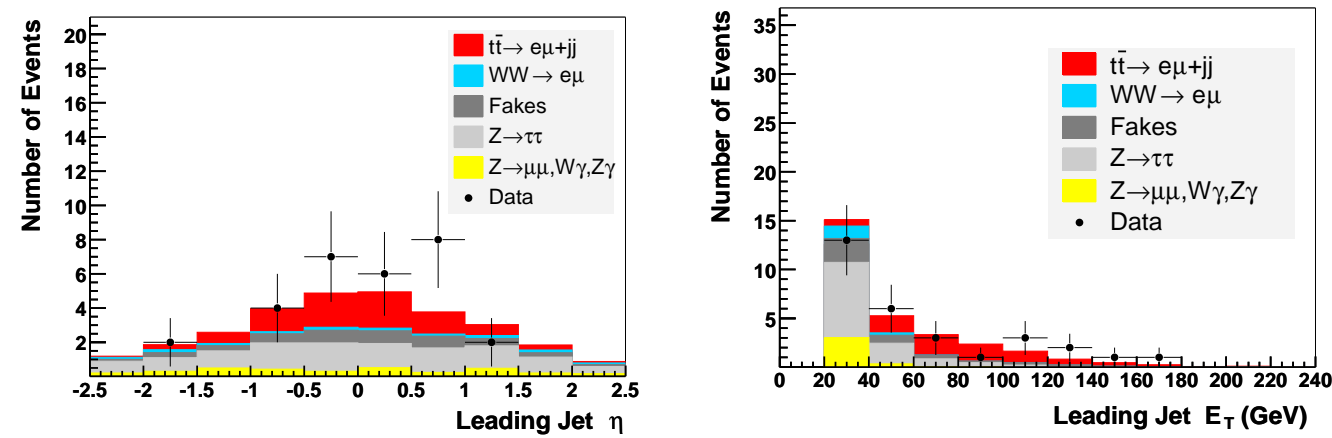

Figure 5.16: Distributions of $\eta$ (left), $p_{T}$ (right) of the leading jet in $t \bar{t} \rightarrow e \mu$, background and data events having one good electron and one good muon with $\Delta R(e, \mu)>0.25$. 

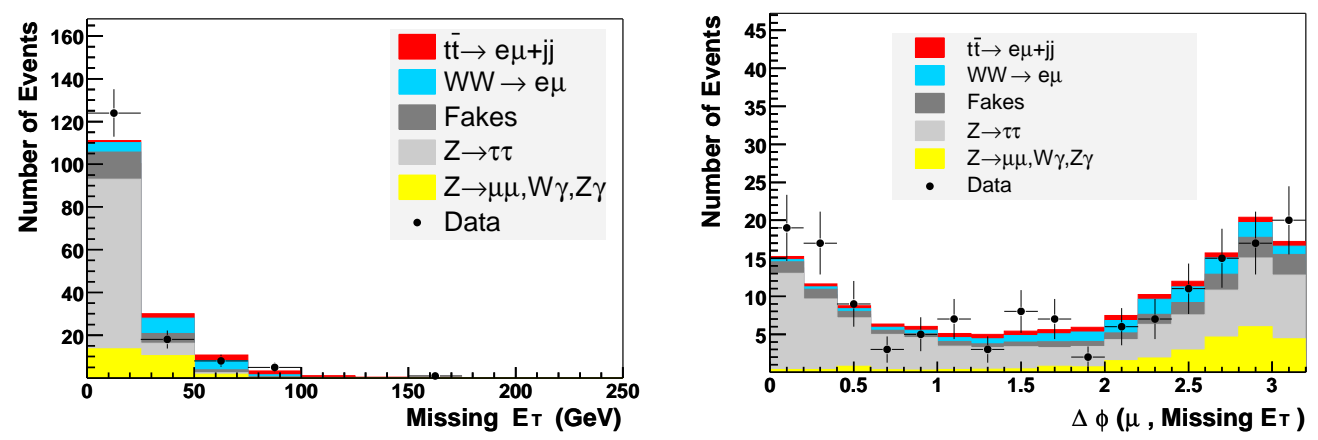

Figure 5.17: Distributions of the $\mathbb{E}_{T}$ (left) and $\Delta \phi\left(\mu, E_{T}\right)$ (right) in $t \bar{t} \rightarrow e \mu$, background and data events having one good electron and one good muon with $\Delta R(e, \mu)>0.25$.
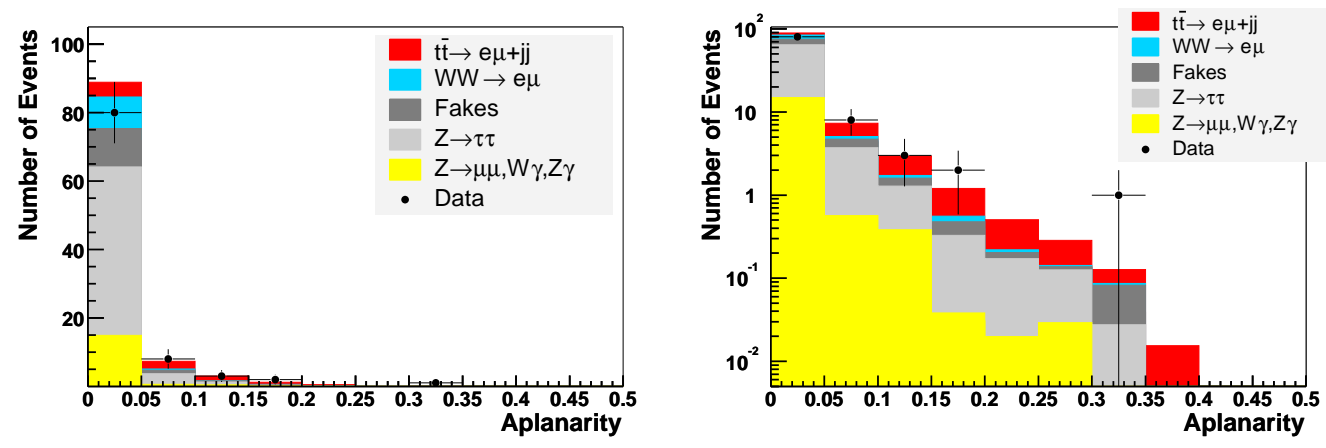

Figure 5.18: Distributions of the aplanarity in linear (left) and log (right) scale in $t \bar{t} \rightarrow e \mu$, background and data events having one good electron and one good muon with $\Delta R(e, \mu)>0.25$. 

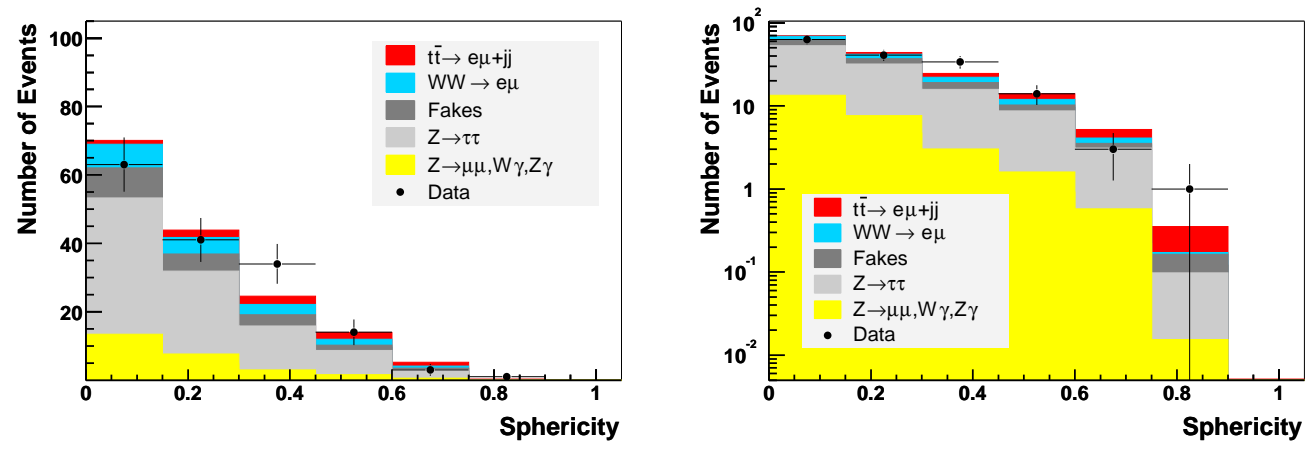

Figure 5.19: Distributions of the sphericity in linear (left) and log (right) scale in $t \bar{t} \rightarrow e \mu$, background and data events having one good electron and one good muon with $\Delta R(e, \mu)>0.25$.
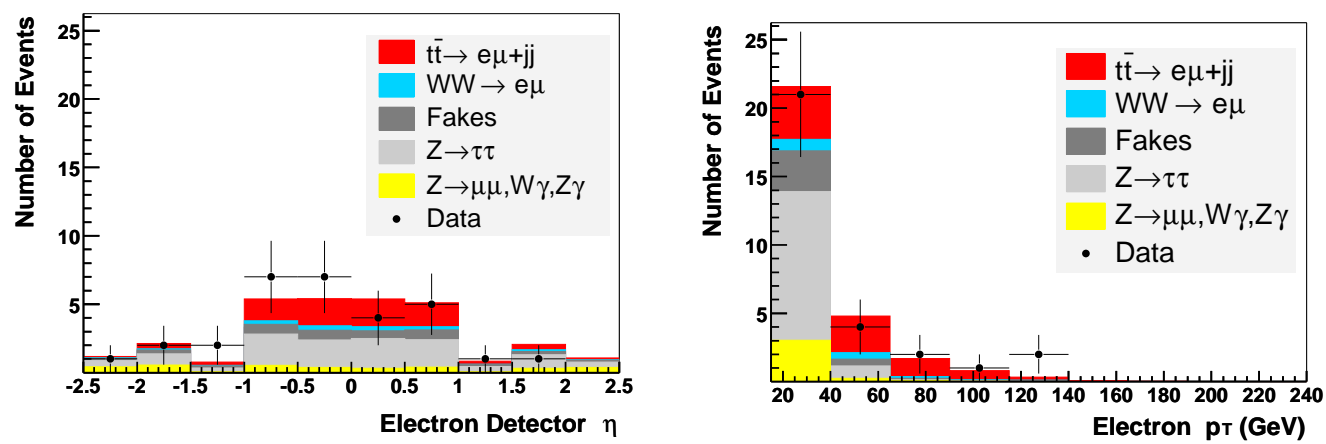

Figure 5.20: Distributions of the detector $\eta$ (left) and $p_{T}$ (right) of electrons in $t \bar{t} \rightarrow e \mu$, background and data events having one good electron and one good muon with $\Delta R(e, \mu)>0.25$, and $\geq 1$ jet with $p_{T}>20 \mathrm{GeV}$. 


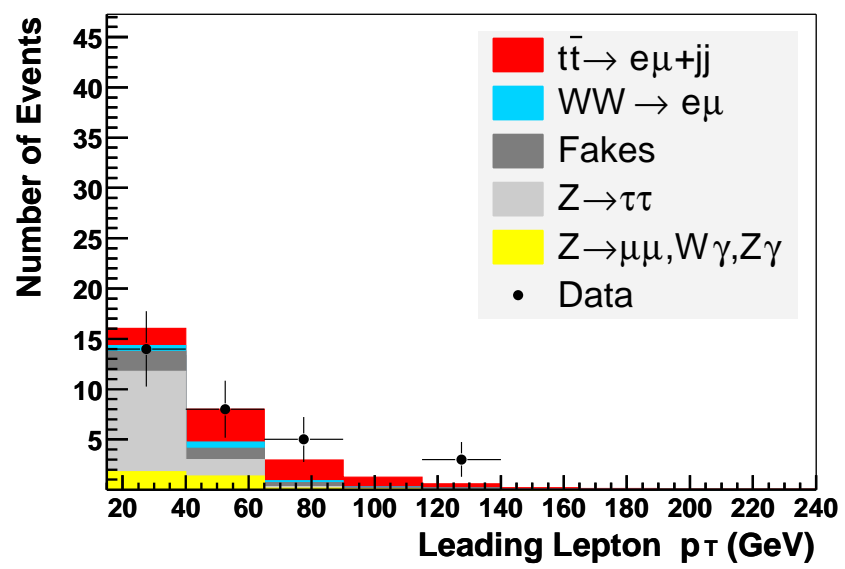

Figure 5.21: Distribution of $p_{T}$ of the leading lepton in $t \bar{t} \rightarrow e \mu$, background and data events having one good electron and one good muon with $\Delta R(e, \mu)>0.25$, and $\geq 1$ jet with $p_{T}>20 \mathrm{GeV}$.
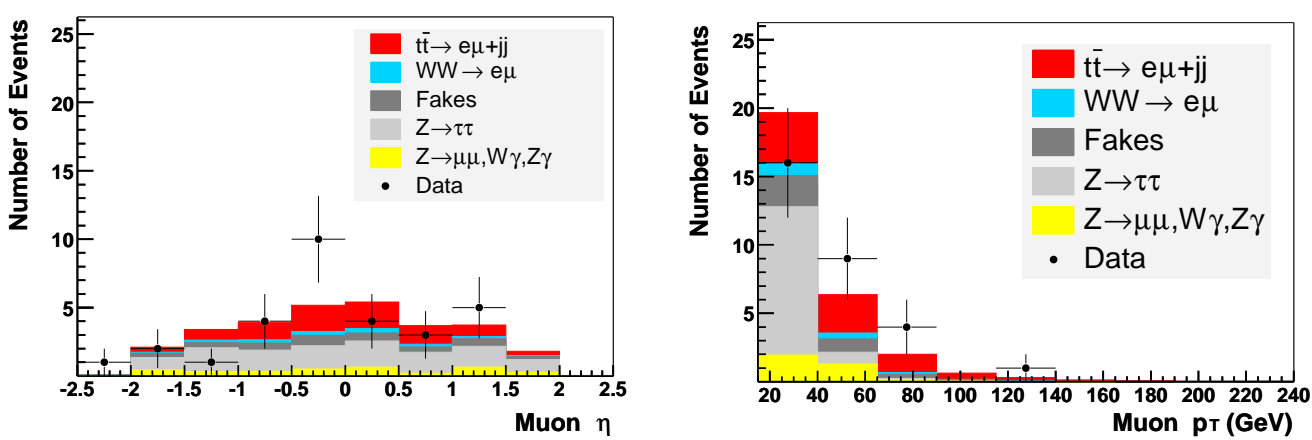

Figure 5.22: Distributions of $\eta$ (left), $p_{T}$ (right) of muons in $t \bar{t} \rightarrow e \mu$, background and data events having one good electron and one good muon with $\Delta R(e, \mu)>0.25$, and $\geq 1$ jet with $p_{T}>20 \mathrm{GeV}$. 

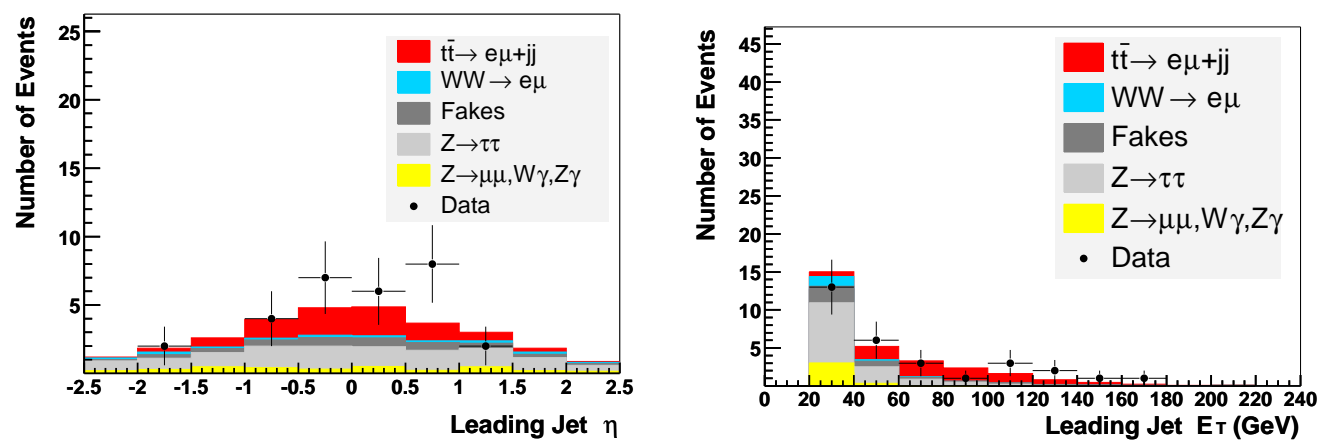

Figure 5.23: Distributions of $\eta$ (left), $p_{T}$ (right) of the leading jet in $t \bar{t} \rightarrow e \mu$, background and data events having one good electron and one good muon with $\Delta R(e, \mu)>0.25$, and $\geq 1$ jet with $p_{T}>20 \mathrm{GeV}$.
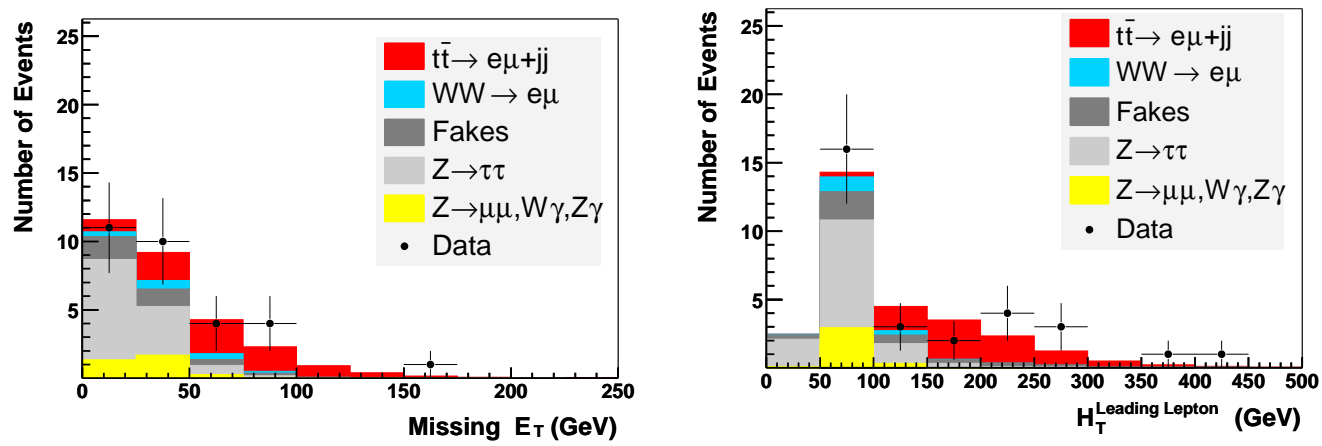

Figure 5.24: Distributions of the $\mathbb{E}_{T}$ (left), $H_{T}^{\text {leading lepton }}$ (right) in $t \bar{t} \rightarrow e \mu$, background and data events having one good electron and one good muon with $\Delta R(e, \mu)>0.25$, and $\geq 1$ jet with $p_{T}>20 \mathrm{GeV}$. 

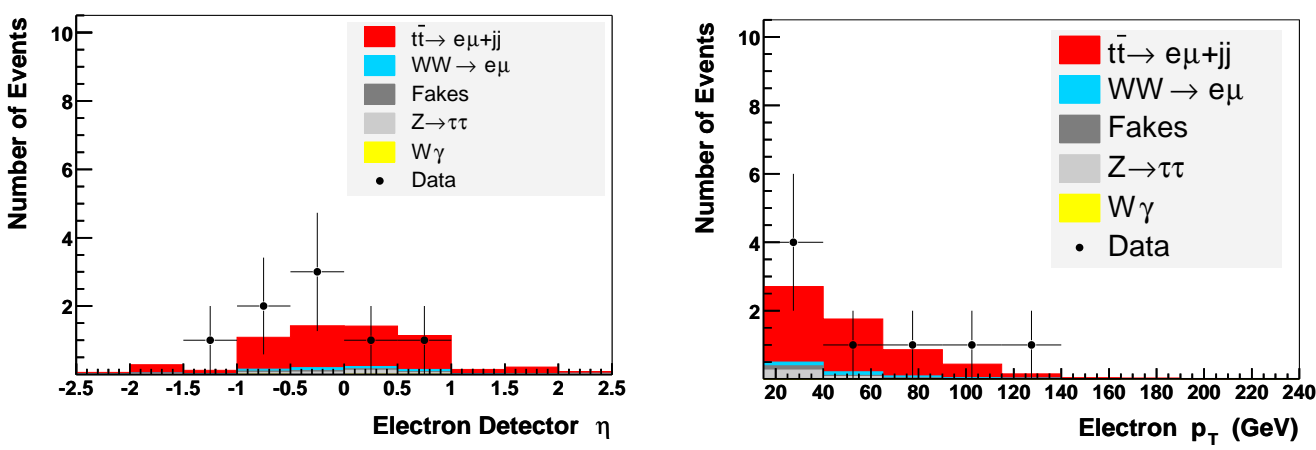

Figure 5.25: Distributions of detector $\eta$ (left) and $p_{T}$ (right) of electrons in $t \bar{t} \rightarrow e \mu$, background and data events after applying all selection criteria (see Tab. 5.1).
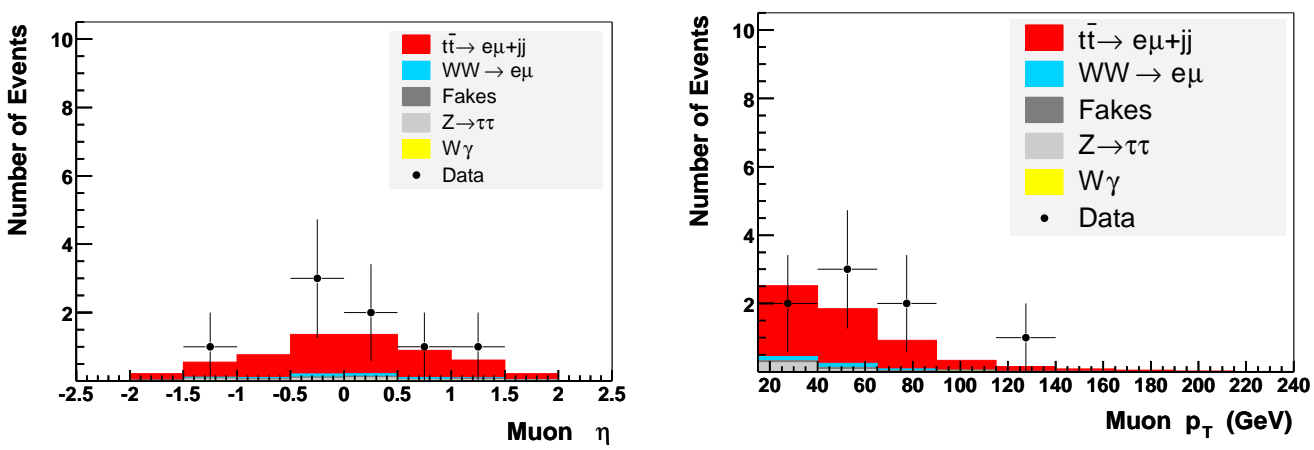

Figure 5.26: Distributions of $\eta$ (left) and $p_{T}$ (right) of muons in $t \bar{t} \rightarrow e \mu$, background and data events after applying all selection criteria (see Tab. 5.1). 

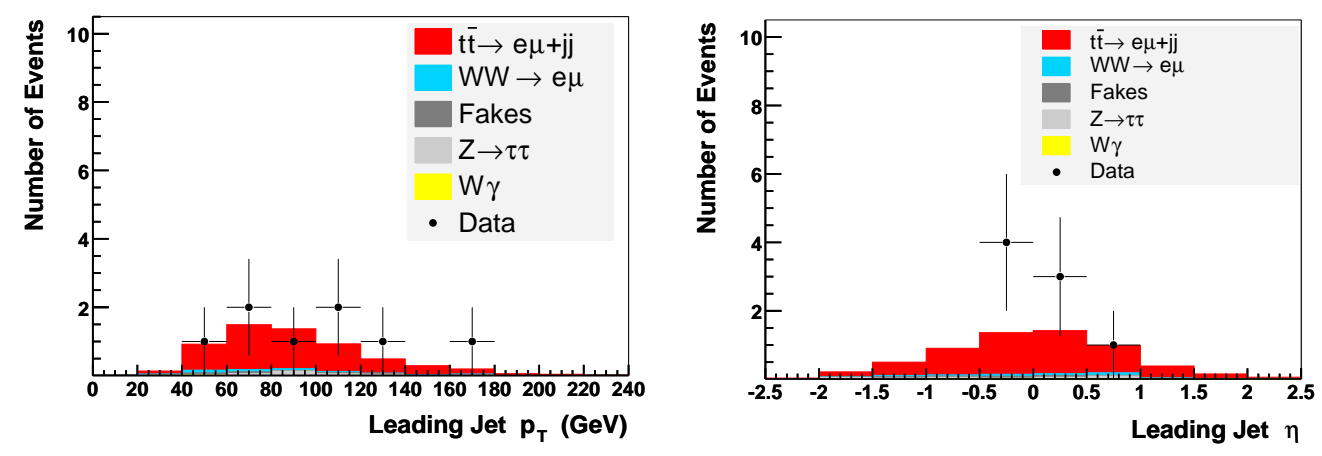

Figure 5.27: Distributions of $p_{T}$ (left) and $\eta$ (right) of the leading jet in $t \bar{t} \rightarrow e \mu$, background and data events after applying all selection criteria (see Tab. 5.1).

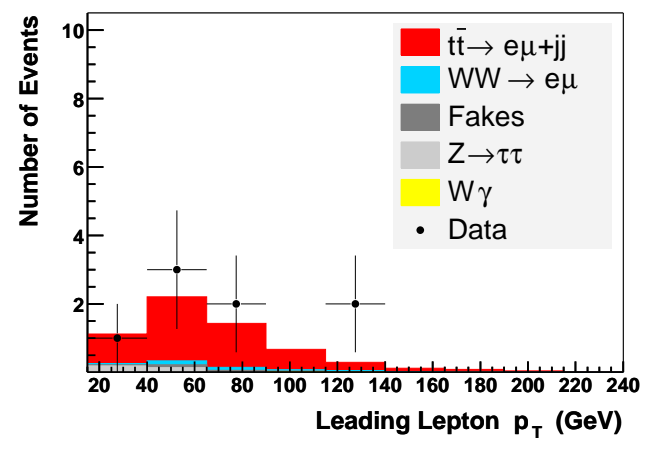

(a)

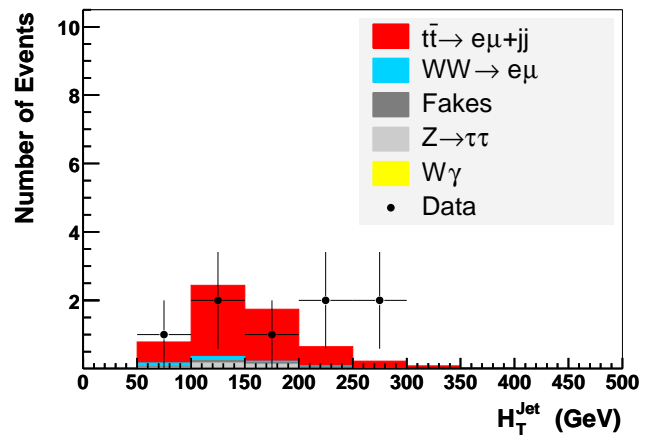

(b)

Figure 5.28: Distribution of (a) $p_{T}$ of the leading lepton and (b) $H_{T}^{\text {jet }}$ in $t \bar{t} \rightarrow e \mu$, background and data events after applying all selection criteria (see Tab. 5.1). 

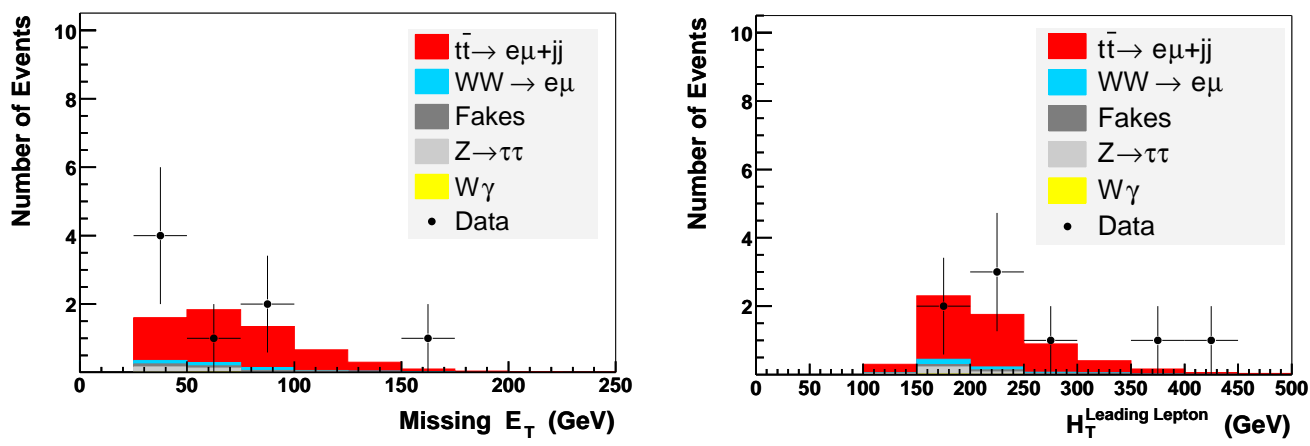

Figure 5.29: Distributions of $\not_{T}$ (left), $H_{T}^{\text {leading lepton }}$ (right) in $t \bar{t} \rightarrow e \mu$, background and data events after applying all selection criteria (see Tab. 5.1).

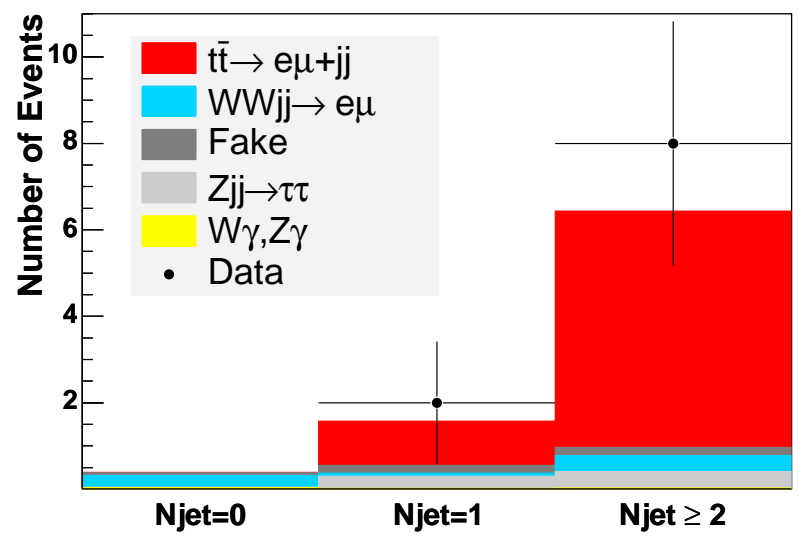

Figure 5.30: Distributions of number of jets with $p_{T}>20 \mathrm{GeV}$ in $t \bar{t} \rightarrow e \mu$, background and data events after applying all selection criteria (see Tab. 5.1). 


\begin{tabular}{|c|c|}
\hline Run Number & Event Number \\
\hline \hline 178733 & 8735139 \\
\hline 179141 & 11709332 \\
\hline 179195 & 26386170 \\
\hline 178159 & 37315438 \\
\hline 177826 & 15259654 \\
\hline 179331 & 19617819 \\
\hline 174901 & 8710859 \\
\hline 168393 & 1997007 \\
\hline
\end{tabular}

Table 5.19: Run numbers and event numbers for the $e \mu$ candidate events.

\subsection{Systematics}

The signal and background efficiencies estimated in this analysis, are sometimes sensitive to the measurement methodologies, e.g., derivation of fake background from experimental data, estimation of trigger efficiencies, uncertainties in MC event simulation. Such effects are taken into consideration by assigning systematic uncertainties on the relevant measurements/methodologies. Moreover, derivation of various scale factors which are described in previous sections can also give rise to systematic uncertainties to be applicable to the present analysis. A description of the sources of these uncertainties is presented below. The values of these uncertainties on signal and background processes are quoted in Tab. 5.20. 


\section{PV Scale Factors}

1. The uncertainty associated with the scale factor for the primary vertex selection i.e., with the selection criteria " $\left|\mathrm{Z}_{\mathrm{PV}}\right|<60 \mathrm{~cm} \mathrm{AND} \mathrm{N}_{\text {trk }} \geq$ 3", arises due to the limited statistics in the data and Monte Carlo samples used to determine the scale factor (see Sec. 5.3.1). A systematic uncertainty of $0.3 \%$ (from Tab. 5.3) is considered here.

2. The scale factor associated with the requirement, $\mid z_{\mathrm{PV}}(\mathrm{d} \emptyset$ root $)-z_{\mathrm{PV}}$ $(\mathrm{d} \emptyset \mathrm{reco}) \mid<5 \mathrm{~cm} "$ is taken to be equal to unity (see Sec. 5.3.1). The corresponding systematic uncertainty is taken to be the largest difference between unity and a measured scale factor (either from $Z^{0} / \gamma^{*} \rightarrow$ ee or $Z^{0} / \gamma^{*} \rightarrow \mu \mu$ samples) for this selection, yielding a systematic uncertainty of $0.1 \%$ (Tab. 5.3).

3. Uncertainty associated with the $\Delta z(P V, e / \mu)$ criteria arises due to the limited statistics of $Z^{0} / \gamma^{*} \rightarrow e e$ and $Z^{0} / \gamma^{*} \rightarrow \mu \mu$ data and Monte Carlo events (see Tab. 5.2 of Sec. 5.3.1). Half of the difference between the scale factors obtained on $Z^{0} / \gamma^{*} \rightarrow e e$ and $Z^{0} / \gamma^{*} \rightarrow \mu \mu$ decays, is taken as a systematic uncertainty. The statistical uncertainty on the 
scale factor is added in quadrature.

$$
\Delta \kappa^{2}=\left(\frac{1}{2}\left|\kappa_{Z^{0} / \gamma^{*} \rightarrow \mu \mu}-\kappa_{Z^{0} / \gamma^{*} \rightarrow e e}\right|\right)^{2}+\Delta \kappa_{Z^{0} / \gamma^{*} \rightarrow \mu \mu}^{2}+\Delta \kappa_{Z^{0} / \gamma^{*} \rightarrow e e}^{2}
$$

where $\kappa_{Z^{0} / \gamma^{*} \rightarrow \mu \mu}\left(\kappa_{Z^{0} / \gamma^{*} \rightarrow e e}\right)$ is the scale factor measured from $Z^{0} / \gamma^{*} \rightarrow$ $\mu \mu\left(Z^{0} / \gamma^{*} \rightarrow e e\right)$ events (both data and Monte Carlo), with a statistical precision of $\Delta \kappa_{Z^{0} / \gamma^{*} \rightarrow \mu \mu}\left(\Delta \kappa_{Z^{0} / \gamma^{*} \rightarrow e e}\right)$. The systematic uncertainty is taken to be $0.1 \%$ for this PV scale factor.

\section{Electron Scale factors}

1. The largest scatter of the scale factor versus $\Delta R(e$, jet $)$ is taken to be the systematic uncertainty associated with electron reconstruction and identification scale factor, as quoted in Tab. 5.4.

2. Uncertainty associated with the electron track match and likelihood scale factor arises from the $\eta$ and $\phi$ dependence of the associated scale factor (see Sec. 5.3.2). In CC, the systematic error on this scale factor is taken as the RMS of the scale factor as functions of $\eta$ and $\phi$. In the EC, additional statistical uncertainty comes from the limited size of the $t \bar{t} \rightarrow e \mu$ and $t \bar{t} \rightarrow e e \mathrm{MC}$ samples, as the scale factor for EC electrons is derived using these samples. 


\section{Muon Scale factors}

1. Uncertainty associated with the muon reconstruction and identification scale factor is obtained by varying the scale factor by its statistical error and is found to have a value of $4 \%$.

2. The largest component of the systematic uncertainty associated with muon track matching scale factor arises due to its $\eta$ and $\phi$ dependence (see Ref. [91]). The effect of contamination of $Z^{0} / \gamma^{*} \rightarrow \mu \mu$ data sample (used to estimate the track matching efficiency for data events) by cosmic muons or muons from semileptonic decays, is also a major source of uncertainty for this scale factor. Adding the contributions from these two sources in quadrature provides a systematic uncertainty of $3 \%$ for muon track match scale factor.

3. Uncertainty associated with the muon isolation scale factor is the statistical uncertainty on its measured value (see Tab. 5.6), i.e. $0.4 \%$.

4. The systematic uncertainty associated with muon DCA significance scale factor is taken from its statistical uncertainty, yielding a value of $0.3 \%$ (see Tab. 5.5)

5. Uncertainty associated with the muon track $\chi^{2}$ scale factor is considered 
to be $0.1 \%$, obtained from the statistical error on the scale factor (see Tab. 5.7).

\section{Jets}

1. The effect of the jet energy scale uncertainty is obtained through varying the JES correction factor (see Sec. 3.2.5) by $\pm 1 \sigma$, where

$$
\sigma=\sqrt{\sigma_{\text {stat, data }}^{2}+\sigma_{\text {syst, data }}^{2}+\sigma_{\text {stat, Monte Carlo }}^{2}+\sigma_{\text {stat, Monte Carlo }}^{2}} .
$$

This is the largest systematic uncertainty among all uncertainties discussed here.

2. The uncertainty arising from jet energy resolution in data is already included in the systematic uncertainty due to the jet energy scale, while the energy of jets in Monte Carlo events are smeared to the jet energy resolution in data events. Therefore, the parameters used to smear the jet energies in Monte Carlo (see Sec. 4.3.3), are varied by $\pm 1 \sigma$ to obtain the systematic uncertainty due to the jet energy resolution for the Monte Carlo events.

3. The scale factor associated with the jet reconstruction and identifica- 
tion efficiency is $E_{T}$ dependent and is derived by dividing the probability to reconstruct and identify a jet in data by the same probability in Monte Carlo (see Sec. 5.3.4). The $E_{T}$ dependent scale factor suffers from a number of uncertainties arising from the method used. First of all the $\gamma+$ jet sample has limited statistics and thus provides a $\pm 1 \sigma$ band for the scale factor versus $E_{T}$. Selection criteria used to select $\gamma+$ jet events in data can be varied in order to measure the uncertainty on the method of selection. It is found that the resulting variation of the scale factor is contained within the statistical $\pm 1 \sigma$ band. The scale factor for jet reconstruction and identification can also be derived using $Z^{0}+$ jet data and Monte Carlo samples. The scale factor obtained in this way has a large statistical uncertainty, but is consistent within errors of the scale factor derived using $\gamma+$ jet samples. By construction, the scale factor derived from the $\gamma+$ jet samples is a function of the $E_{T}$ of the photon rather than that of the jet. On the other hand when the scale factor is applied on a Monte Carlo sample, the $E_{T}$ of the jet is used to choose the value of the scale factor. This is not satisfying since the $E_{T}$ resolution is different for jets and photons. The potential size of this effect on the scale factor is estimated by smearing the $E_{T}$ of the photon with the energy resolution for jets. The resulting smeared 
scale factor is well within the statistical $\pm 1 \sigma$ band of the scale factor (from $\gamma+$ jet sample). In conclusion, the systematic uncertainty arising from the jet reconstruction and identification scale factor on the $t \bar{t}$ and background efficiencies is estimated by varying the scale factor parametrization between the $+1 \sigma$ and the $-1 \sigma$ parameterizations.

\section{Trigger Efficiency}

The efficiencies of electron and muon triggers are determined using the $Z^{0} / \gamma^{*} \rightarrow$ $e e$ and $Z^{0} / \gamma^{*} \rightarrow \mu \mu$ data samples. The uncertainty on the trigger efficiency is taken from the statistical errors on the turn on curves which are limited by the $Z^{0} / \gamma^{*} \rightarrow e e$ or $Z^{0} / \gamma^{*} \rightarrow \mu \mu$ statistics. The systematic uncertainty arising from the trigger efficiency is estimated by varying the individual turn on curves by $\pm 1 \sigma$ from its mean value. In other words, each turn on curve has a statistical error band within which they are varied and the deviation from its (total trigger efficiency) mean value is quoted as systematic uncertainty on trigger efficiency.

\section{Top Mass}

The present analysis is based on the Monte Carlo sample generated with top mass of $175 \mathrm{GeV} / \mathrm{c}^{2}$. In order to estimate the effect due to the variation of 


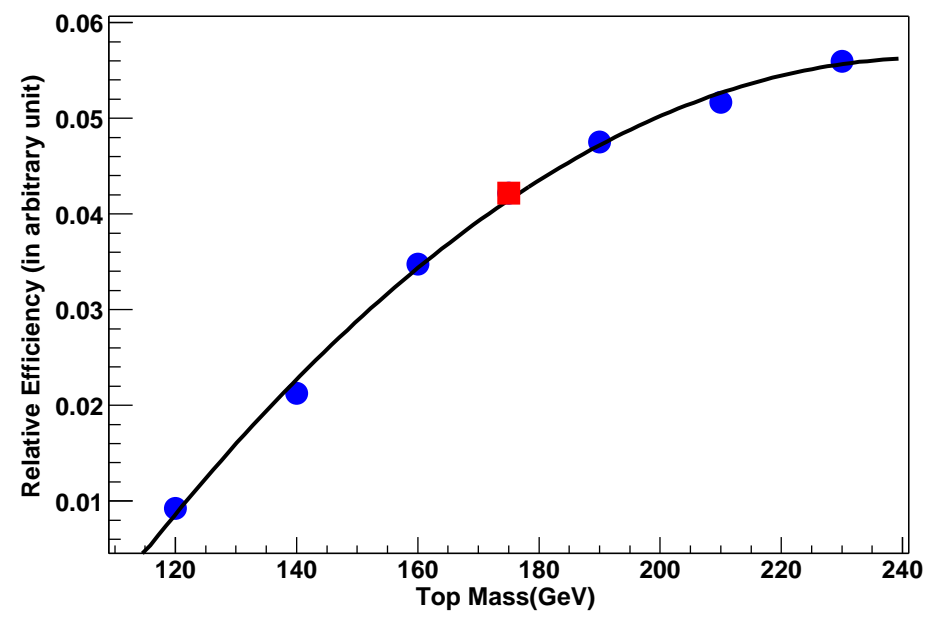

Figure 5.31: The $t \bar{t} \rightarrow e \mu$ event selection efficiency (in arbitrary units) as function of the mass of the top quark. The present analysis is based on a $\mathrm{MC}$ sample with top quark mass of $175 \mathrm{GeV} / \mathrm{c}^{2}$ (shown in red marker).

top mass, Monte Carlo samples are generated with different top masses. A total of seven samples having the top masses $120,140,160,175,190,210$, and $230 \mathrm{GeV} / \mathrm{c}^{2}$ are used. To minimize the impact of statistical fluctuations in any one sample, the $t \bar{t} \rightarrow e \mu$ event selection efficiency versus top mass curve is fitted to a smooth function. The differences in the values of the fit function at $\mathrm{m}_{\mathrm{t}}=170 \mathrm{GeV} / \mathrm{c}^{2}$ and $m_{t}=180 \mathrm{GeV} / c^{2}$ from the corresponding value at $\mathrm{m}_{\mathrm{t}}=175 \mathrm{GeV} / \mathrm{c}^{2}$, are considered to be the lower and upper limits of uncertainties due to top mass. Fig. 5.31 shows the $t \bar{t}$ efficiency as function of the mass of the top quark. 


\section{Theoretical cross sections}

For the $W^{+} W^{-}$and $W^{ \pm} \gamma$ background processes theoretical cross sections are used for normalization (see Sec. 4.3.1). The leading order $W^{+} W^{-}$production cross section differs by $35 \%$ from the NLO production cross section. Such a theoretical calculation (NLO) does not exist for $W^{+} W^{-}+2 j e t$ process; so a $35 \%$ systematic uncertainty is introduced to take into account this possible source of error for $W^{+} W^{-}$background processes.

The production cross section of the $W^{ \pm} \gamma$ process is an experimentally measured quantity, in agreement with theory. But the measured cross section is for a certain $E_{T}$ cut on the photon and for a minimum opening angle

between the muon from the $W^{ \pm}$decay and the photon. The opening angle, $\Delta R(\mu, \gamma)$ cut is varied between 0.15 and 0.35 and the difference is taken as the systematic uncertainty.

\section{Photon background}

As described in Sec. 5.5.2, the photon track matching rate in MC is not in good agreement with that in experimental data. One of the most recent studies have revealed that improper modelling of the detector geometry in DØ GEANT, leads to a $20 \%$ difference in photon conversion rates between 
data and Monte Carlo. On the basis of this study, a systematic uncertainty of $20 \%$ is assigned to the photon background processes in the current analysis.

\section{Instrumental Fake}

As shown in Tab. 5.16 of Sec. 5.5.3, two approaches in estimating the instrumental fake rates give rise to a difference of $39 \%$, which is considered to be the systematic uncertainty in estimation of fake background events for the present analysis. 


\begin{tabular}{|l||c|c|c|c|}
\hline \hline \multicolumn{1}{|l||}{ Source } & Signal & \multicolumn{3}{|c|}{ Backgrounds } \\
\hline & $t \bar{t} \rightarrow e \mu$ & $Z^{0} / \gamma^{*} \rightarrow \tau \tau$ & $W^{+} W^{-}$ & $W^{ \pm} \gamma$ \\
\hline Primary vertex & \pm 0.3 & \pm 0.3 & \pm 0.3 & \pm 0.3 \\
$\Delta z$ d $\emptyset$ reco, d $\emptyset$ root $)$ & \pm 0.1 & \pm 0.1 & \pm 0.1 & \pm 0.1 \\
Lepton promptness & \pm 0.1 & \pm 0.1 & \pm 0.1 & \pm 0.1 \\
\hline EM reconstruction & \pm 2.5 & \pm 2.5 & \pm 2.5 & \pm 2.7 \\
EM tracking and likelihood & \pm 1.9 & \pm 1.9 & \pm 1.9 & \pm 2.1 \\
\hline L1 EM trigger & \pm 0.02 & \pm 0.06 & \pm 0.06 & \pm 0.1 \\
L3 EM trigger & \pm 0.5 & \pm 0.6 & \pm 1.2 & \pm 1.5 \\
\hline$\mu$ ID & \pm 4.0 & \pm 4.0 & \pm 4.0 & \pm 4.0 \\
$\mu$ isolation & \pm 0.4 & \pm 0.4 & \pm 0.4 & \pm 0.4 \\
$\mu$ dca significance & \pm 0.3 & \pm 0.3 & \pm 0.3 & \pm 0.3 \\
$\mu$ track matching & \pm 3.0 & \pm 3.0 & \pm 3.0 & \pm 3.0 \\
$\mu$ track $\chi^{2}$ & \pm 0.1 & \pm 0.1 & \pm 0.1 & \pm 0.1 \\
\hline L1 $\mu$ trigger & $+1.9-2.5$ & $+3.0-3.9$ & $+2.7-3.5$ & $+3.0-3.7$ \\
\hline JES & $+6.5-5.7$ & $+28.0-17.7$ & $18.6-13.9$ & $+30.0-20.0$ \\
Jet ID & $+5.2-1.3$ & +8.6 & $+7.3-9.8$ & -66.6 \\
Jet energy resolution & +2.7 & +13.7 & -7.3 & -66.6 \\
\hline Jet firing EM trigger & $+0.3-0.8$ & $+0.5-0.3$ & $+0.6-0.3$ & $+2.0-0.0$ \\
\hline Top mass & $+4.9-5.4$ & $\mathrm{~N} / \mathrm{A}$ & $\mathrm{N} / \mathrm{A}$ & $\mathrm{N} / \mathrm{A}$ \\
\hline Theoretical cross section & $\mathrm{N} / \mathrm{A}$ & \pm 35 & $\mathrm{~N} / \mathrm{A}$ & \pm 12.3 \\
\hline$\gamma$ Track matching & $\mathrm{N} / \mathrm{A}$ & $\mathrm{N} / \mathrm{A}$ & $\mathrm{N} / \mathrm{A}$ & \pm 20.6 \\
\hline \hline Uncorrelated (statistical) & \pm 2.1 & \multicolumn{4}{|c|}{ \pm} \\
\hline \hline
\end{tabular}

Table 5.20: Summary of the relative systematic uncertainties (in \%) on signal and different background processes after applying all selection criteria mentioned in Tab. 5.1. The estimate for instrumental fake events has a $39 \%$ uncertainty on its value which is not quoted here, as the instrumental fake events of this analysis are directly estimated from data. 


\subsection{Cross section calculation}

In order to estimate the cross section, a maximum likelihood method has been utilized. Poisson hypothesis is considered to account for the small number of observed events. The likelihood of $N^{\mathrm{Obs}}$ events, given the hypothesis of $\tilde{N}$ $\left(\equiv \sigma \cdot B R \cdot L^{\text {integrated }} \cdot \epsilon+N^{\mathrm{bkg}}\right)$ is defined as,

$$
\mathcal{Q}\left(\sigma,\left\{N^{\text {Obs }}, N^{\text {bkg }}, \mathrm{BR}, L^{\text {integrated }}, \epsilon\right\}\right)=\frac{\tilde{N}^{N^{\text {Obs }}}}{N^{\text {Obs! }}} \cdot e^{-\tilde{N}}
$$

where $N^{\mathrm{Obs}}, N^{\mathrm{bkg}}, L^{\text {integrated }}, B R$ and $\epsilon, \sigma$ are respectively the number of observed events, the number of background events, integrated luminosity, the branching fraction of $t \bar{t} \rightarrow e \mu$ (including $\tau$-contributions), the overall signal efficiency and $p \bar{p} \rightarrow t \bar{t}$ production cross section. The relevant numbers which are used in the cross section calculation, are tabulated in Tab. 5.21.

\begin{tabular}{|c|c|}
\hline \hline Quantity & Value \\
\hline$N^{\text {Obs }}$ & 8 \\
$N^{\text {bkg }}$ & $0.91_{-0.17}^{+0.20}$ \\
$\mathrm{BR}(t \bar{t} \rightarrow e \mu)$ & 0.03155 \\
$L^{\text {integrated }}$ & $228.29 \mathrm{pb}^{-1}$ \\
$\epsilon$ (Signal efficiency) & $0.1020 \pm 0.0022$ \\
\hline \hline
\end{tabular}

Table 5.21: Values of the variables which are utilised to estimate the $t \bar{t}$ production cross section in $e \mu$ channel. 
The cross section is then estimated by minimising the quantity

$$
-2 \ln \mathcal{Q}\left(\sigma,\left\{N^{\mathrm{Obs}}, N^{\mathrm{bkg}}, \mathrm{BR}, L^{\text {integrated }}, \epsilon\right\}\right)
$$

The distribution of $-2 \ln \mathcal{Q}$ as a function of $t \bar{t}$ production cross section is shown in Fig. 5.32. Minima of the distribution provides the mean value of the measured cross section as,

$$
\sigma(p \bar{p} \rightarrow t \bar{t})=\frac{N^{\text {Obs }}-N^{\mathrm{bkg}}}{L^{\text {integrated }} \cdot \epsilon \cdot B R(t \bar{t} \rightarrow e \mu)}
$$

The statistical error on measured cross section is estimated by varying $-2 \ln \mathcal{Q}$ by one unit from its minima. The systematic error on the cross section is estimated by varying the signal efficiency and background contribution within their systematic uncertainties tabulated in Tab. 5.20. Since luminosity is measured independently by the $\mathrm{D} \varnothing$ luminosity monitors with an accuracy of $6.5 \%$, it is assumed to be an uncorrelated source of systematic error on the measured cross section.

Applying the procedure mentioned above, the $t \bar{t}$ production cross sec- 


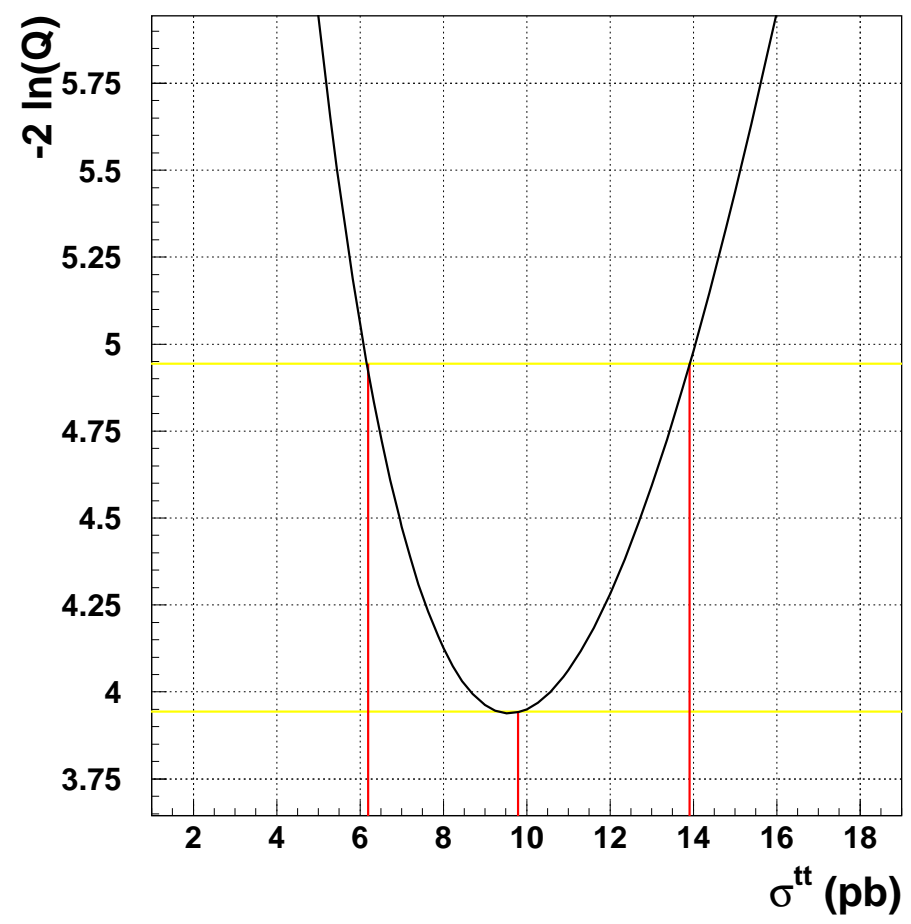

Figure 5.32: Likelihood as a function of the $t \bar{t}$ production cross section. The central value and the statistical errors are indicated as vertical line.

tion in the $e \mu$ decay channel is found to be,

$$
9.7_{-3.4}^{+4.3} \text { (stat) }{ }_{-1.3}^{+1.2} \text { (syst) } \pm 0.6 \text { (lumi) pb. }
$$

The measurement suffer from a large statistical uncertainty due to the small number of observed events. Therefore, the probability that the observed events arise from the statistical fluctuation of the background pro- 
cesses can be estimated as

$$
\mathcal{P}\left(\mathrm{N}^{\mathrm{Obs}}, \tilde{\mathrm{b}}\right)=\frac{\tilde{\mathrm{b}}^{\mathrm{N}} \mathrm{e}^{\mathrm{Obs}} \mathrm{e}^{-\tilde{\mathrm{b}}}}{\mathrm{N}^{\mathrm{Obs} !}}
$$

where the mean of expected background events $\tilde{b}$, is assumed to have Poisson fluctuation. The Poisson probability corresponding to the values estimated in the present analysis i.e. $\tilde{b}=0.91_{-0.18}^{+0.21}$ with $\mathrm{N}^{\mathrm{Obs}}=8$, is $4.7 \times 10^{-6}$. Therefore, the probability that the observed events are due to background fluctuation, is ruled out with $>4 \sigma$ confidence level.

Similarly, a gedanken experiment can be performed by taking into consideration the systematic uncertainties in the signal and background estimations of this analysis. In such an experiment, the signal ( $\tilde{\mathrm{s}})$ and background (ש̃) estimations are assumed to follow Poisson distribution, while their uncertainties are assumed to have Gaussian distributions. In other words, the number of signal or background events are randomly generated with a Poisson probability distribution function

$$
\mathcal{P}(\mathrm{x}, \mathrm{a})=\frac{\mathrm{a}^{\mathrm{x}} \mathrm{e}^{-\mathrm{a}}}{\mathrm{x} !}
$$

where $\mathrm{a}=\tilde{\mathrm{s}}(\tilde{\mathrm{b}})$ i.e., the expected number of signal (background) events. 
In order to take the systematic uncertainties in signal $(\Delta \tilde{s})$ and background $(\Delta \tilde{b})$ event estimation into account, both $\tilde{\mathrm{s}}$ and $\tilde{\mathrm{b}}$ are randomly generated with Gaussian probability distribution functions $\mathrm{G}(\mathrm{x}, \tilde{\mathrm{s}}, \Delta \tilde{\mathrm{s}})$ and $\mathrm{G}(\mathrm{x}, \tilde{\mathrm{b}}, \Delta \tilde{\mathrm{b}})$ respectively, where

$$
\mathrm{G}(\mathrm{x}, \mathrm{a}, \Delta \mathrm{a})=\exp \left(-\frac{(\mathrm{x}-\mathrm{a})^{2}}{2 \Delta \mathrm{a}^{2}}\right)
$$

The result of the toy MC experiment is shown in Fig. 5.33. The probability that one observes $\geq 8$ events, is more than $27 \%$, which is significantly high observation probability. If one repeats the same experiment with the dilepton cross section of $8.6 \mathrm{pb}$, as measured by $\mathrm{D} \varnothing$ [93] for signal event estimation, the corresponding probability is $43 \%$. 


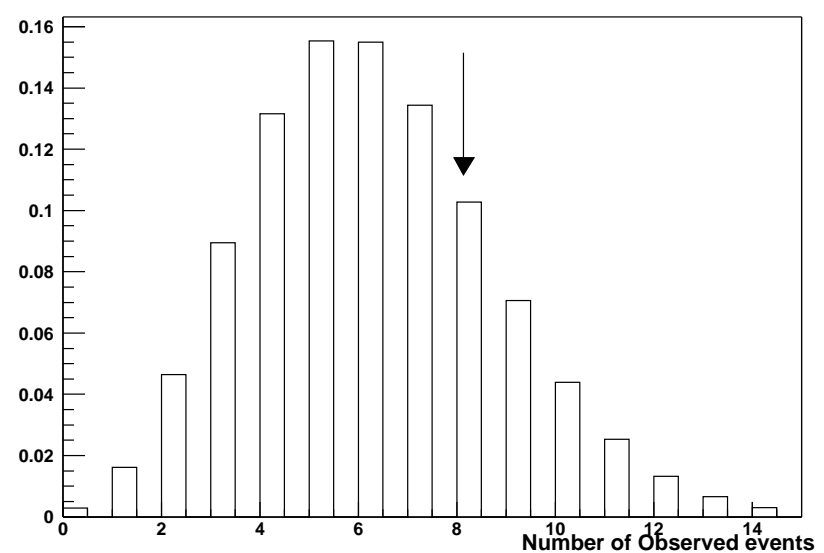

Figure 5.33: Results of a toy Monte Carlo experiment with $5.14 \pm 0.58$ signal (with a $t \bar{t}$ production cross section of $7 \mathrm{pb}$ ) and $0.91 \pm 0.20$ background events. The observed number of events i.e., 8 is shown with the arrow. 


\section{Conclusion}

The latest theoretical (NNLO) calculations using perturbative QCD predict a $t \bar{t}$ production cross section of $6.77 \pm 0.42 \mathrm{pb}$ at $\sqrt{s}=1.96 \mathrm{TeV}$, for a top quark mass of $175 \mathrm{GeV} / \mathrm{c}^{2}$. The measured value presented in this dissertation is

$$
9.7_{-3.4}^{+4.3} \text { (stat) }{ }_{-1.3}^{+1.2} \text { (syst) } \pm 0.6 \text { (lumi) pb, }
$$

using $228.29 \mathrm{pb}^{-1}$ of $\mathrm{D} \emptyset$ data. The mean value obtained from theoretical calculations, is within $1 \sigma$ from the mean of experimentally measured cross section in $e \mu$ channel. Therefore, the $t \bar{t}$ production cross section measured in the $e \mu$ channel is consistent with theoretical prediction. The measured cross section in $e \mu$ channel, as well as the combined dilepton cross section [93] $\left(8.6_{-2.7}^{+3.2}\right.$ (stat) ${ }_{-1.1}^{+1.1}($ syst $) \pm 0.6$ (lumi) pb) are shown in Fig. 1.

To verify the sensitivity of the current measurement in the dilepton 


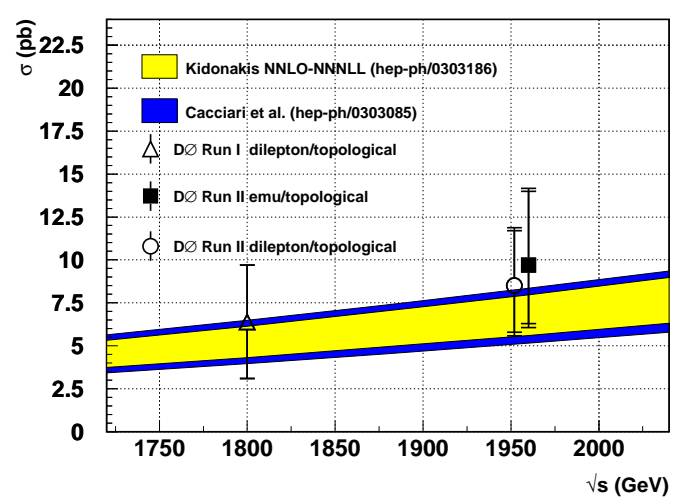

Figure 1: DØ Run II $t \bar{t}$ production cross section as measured in $e \mu$ channel, along with the combined dilepton $(e e+e \mu+\mu \mu)$ cross section [93] is compared to the combined dilepton cross section from Run I [94]. Theoretical calculations by Kidonakis (inner yellow band) and Cacciari et al. (outer blue band) is also shown as a function of centre of mass energy $(\sqrt{s})$.

channel, one can compare the results with the measurement performed by DØ using $\sim 100 \mathrm{pb}^{-1}$ of data during Tevatron Run I [94] i.e., $6.02 \pm 3.21$ pb. As expected, the current dilepton measurement has achieved nearly $40 \%$ improvement in terms of statistical precision over the Run I measurement. This is primarily due to the fact that the current measurement is based on a larger dataset and also due to $30 \%$ increase in the $t \bar{t}$ production cross-section at $\sqrt{s}=1.96 \mathrm{TeV}$ over Run I i.e., at $\sqrt{s}=1.8 \mathrm{TeV}$.

Similarly, one can check the consistency of the $t \bar{t}$ production cross section measured in dilepton final states with those measured by $\mathrm{D} \varnothing$ in other decay channels. All $t \bar{t}$ cross section measurements published so far by $\mathrm{D} \emptyset$ 
based on Tevatron Run II data, are shown in Fig. 2. Both measurements performed in lepton+jets channels viz., topological [95] and vertex tag [96], have better sensitivity compared to that performed in dilepton channel due to the larger number of observed $t \bar{t}$ events. The combined topological cross section [97] i.e., combination of dilepton and lepton+jets(topological) channels agrees quite well with theoretical prediction. In order to validate the sensitivity of the $\mathrm{D} \varnothing$ measured $t \bar{t}$ production cross section in dilepton final states, the same results reported by CDF [98] should be compared. With almost same the amount of dataset, CDF measures a $t \bar{t}$ production cross section of $7.0_{-2.1}^{+2.4}$ (stat) ${ }_{-1.1}^{+1.6}$ (syst) \pm 0.4 (lumi) pb, using dilepton final states. Thus, both the results by CDF and DØ are consistent with each other and they have almost the identical sensitivity in terms of relative statistical uncertainties.

To summarise, top pair production cross sections in $e \mu$ channel as well as in other channels as measured by D $\varnothing$ and also by CDF, are consistent with the SM predictions but still suffer from considerable statistical uncertainties. Future measurements of the same at the Tevatron along with the combination of all decay channels, are expected to reach the sensitivity of theoretical calculations and thus, top quark physics would provide a quantitative answer to the physics beyond SM. 


\section{D $\varnothing$ Run II}

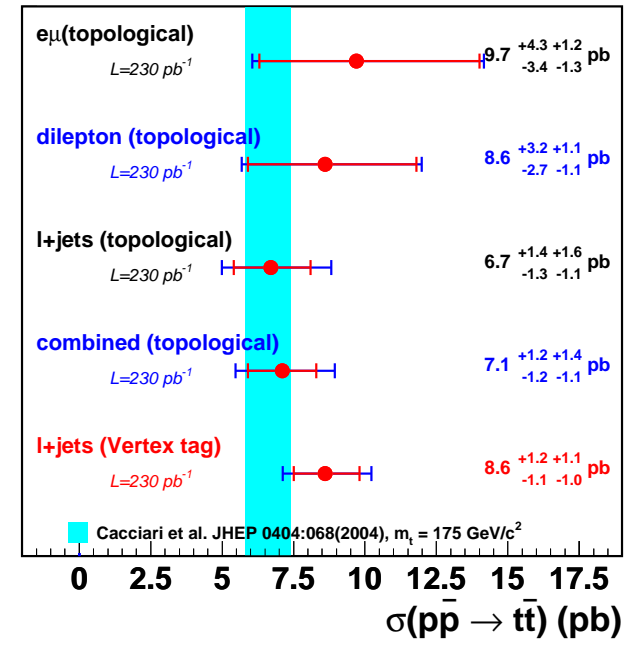

Figure 2: $\mathrm{D} \varnothing$ measured $t \bar{t}$ production cross sections in different decay channels. The band represents the theoretical prediction for the $t \bar{t}$ production cross sections for a top mass of $175 \mathrm{GeV} / \mathrm{c}^{2}$. 


\section{Appendix A}

\section{Topological Variables}

\section{A.1 $\operatorname{Aplanarity}(\mathcal{A})$ and $\operatorname{Sphericity}(\mathcal{S})$}

For each event the normalized momentum tensor (M) [15]can be defined as

$$
M_{a b}=\frac{\sum_{i} p_{i a} p_{i b}}{\sum_{i} p_{i}^{2}}
$$

where $\overrightarrow{p_{i}}$ is the 3 -momentum of the $\mathrm{i}^{\text {th }}$ object in the laboratory frame and $\mathrm{a}, \mathrm{b}, \mathrm{c}$ are the indices for three space dimensions. $\mathrm{M}$ is a symmetric matrix which can be diagonalised. Let the eigenvalues are $\mathrm{Q}_{1}, \mathrm{Q}_{2}$ and $\mathrm{Q}_{3}$ where

$$
0 \leq \mathrm{Q}_{1} \leq \mathrm{Q}_{2} \leq \mathrm{Q}_{3} ; \text { and } \mathrm{Q}_{1}+\mathrm{Q}_{2}+\mathrm{Q}_{3}=1
$$


These eigenvalues are subsequently used to quantify the shape of an event

- for roughly spherical (ball-shaped) events, $\mathrm{Q}_{1} \approx \mathrm{Q}_{2} \approx \mathrm{Q}_{3}$

- for coplanar (pancake-shaped) events, $\mathrm{Q}_{1} \ll \mathrm{Q}_{2}$

- for collinear (cigar-shaped) events, $\mathrm{Q}_{2} \ll \mathrm{Q}_{3}$

Particular combinations of $Q_{j}$ have been given names and discriminate between different physics processes. The sphericity $(\mathcal{S})$ is defined as

$$
\mathcal{S}=\frac{3}{2}\left(\mathrm{Q}_{1}+\mathrm{Q}_{2}\right)=\frac{3}{2} \min \left[\sum_{\mathrm{j}}\left(\mathrm{p}_{\mathrm{jT}}\right)^{2}\right] /\left[\sum_{\mathrm{j}} \mathrm{p}_{\mathrm{j}}^{2}\right]
$$

where the subscript $\mathrm{T}$ denotes the momentum component transverse to an axis which minimizes the sum of the numerator (here it is $\mathbf{n}_{3}$ ); the axis is called sphericity axis. The range of sphericity is $[0,1]$. Events with $\mathcal{S} \approx 1$ are spherical (isotropic distribution) while $\mathcal{S} \ll 1$ bears the signature of a cigar-shaped (back-to-back) event.

The plane of the eigenvectors $\mathbf{n}_{2}$ and $\mathbf{n}_{3}$ is called the event plane. The aplanarity $(\mathcal{A})$ is defined by

$$
\mathcal{A}=\frac{3}{2} \mathrm{Q}_{1}
$$

and lies in the range $0 \leq \mathcal{A} \leq 1 / 2$. One can isolate $t \bar{t}$ (spherical) from QCD (coplanar) events by requiring higher value of $\mathcal{A}$. 


\section{A.2 $\tau \tau$ Invariant mass}

In $Z^{0} \rightarrow \tau \tau \rightarrow e \mu+$ jets events, the large mass of $Z^{0}$ and its large transverse momentum, result in high velocities of $\tau^{\prime}$ 's coming from $Z^{0}$ decay. For these events, the approximation that the leptons viz., electron and muon are close to the parent $\tau$ directions (collinear approximation) can be made. It is possible to extract the fractions of the two $\tau$ momenta which are carried away by the charged leptons. These momenta fractions $x_{\tau_{1}}, x_{\tau_{2}}$, can be expressed, in terms the transverse momenta of the $Z^{0}$, leptons and missing transverse momentum of the event

$$
\begin{array}{r}
p_{\mathrm{T}, \mathrm{Z}^{0}}=p_{\mathrm{T}, \mathrm{e}}+p_{\mathrm{T}, \mu}+\overrightarrow{\not_{T}} \\
x_{\tau_{i}}=\frac{p_{\mathrm{T}, \text { lepton i }}}{p_{\mathrm{T}, \tau_{\mathrm{i}}}}
\end{array}
$$

where $x_{\tau_{i}}$ is the momentum fraction of $\tau_{i}$, carried by the lepton $\mathrm{i}$ and can be rewritten as

$$
\begin{aligned}
& x_{\tau_{1}}=\frac{p_{\mathrm{x}, \text { lepton } 1} \cdot p_{\mathrm{y}, \text { lepton } 2}-p_{\mathrm{y}, \text { lepton } 1} \cdot p_{\mathrm{x}, \text { lepton } 2}}{p_{\mathrm{Tx}, \mathrm{Z}^{0}} \cdot p_{\text {Ty,lepton } 2}-p_{\mathrm{Ty}, \mathrm{Z}^{0}} \cdot p_{\mathrm{x}, \text { lepton } 2}} \\
& x_{\tau_{2}}=\frac{p_{\mathrm{x}, \text { lepton } 1} \cdot p_{\mathrm{y}, \text { lepton } 2}-p_{\mathrm{y}, \text { lepton } 1} \cdot p_{\mathrm{x}, \text { lepton } 2}}{p_{\mathrm{Ty}, \mathrm{Z}^{0}} \cdot p_{\mathrm{Tx}, \text { lepton } 1}-p_{\mathrm{Tx}, \mathrm{Z}^{0}} \cdot p_{y, \text { lepton } 1}}
\end{aligned}
$$




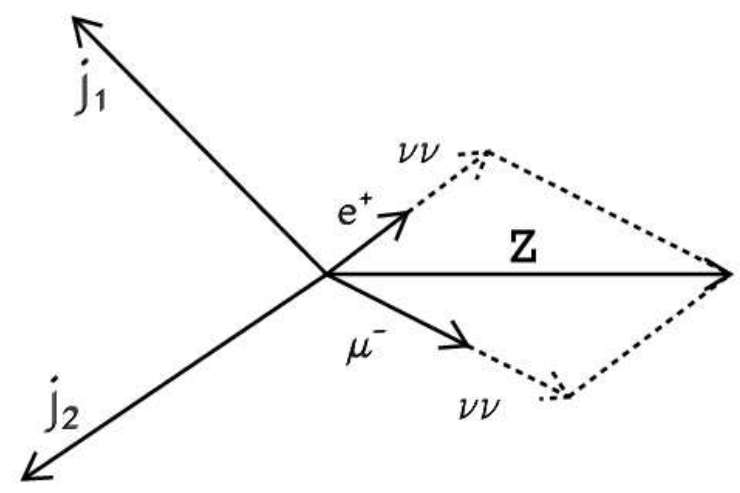

Figure A.1: Schematic view of a $Z^{0} \rightarrow \tau \tau \rightarrow e \mu+$ jets event.

As shown in Fig. A.1, the transverse momentum of the $Z^{0}$ boson, $p_{\mathrm{T}, Z^{0}}$ is given by the vectorial sum of the charged lepton $p_{T}$ 's and the missing transverse momentum carried away by the neutrinos.

The reconstruction of such a momentum fraction works only if the $\tau$ 's are not emitted back-to-back in transverse plane; otherwise the momentum vectors are linearly dependent and the denominator becomes zero. A cut requiring a minimal acoplanarity of the lepton pair

$$
\cos _{e \mu}>-0.9
$$

can be applied to exclude this possibility. For $\tau$ decays, such methodology must yield $0<x_{\tau_{1,2}}<1$ apart from the detector resolution effects. For 
leptons originating from $W W \rightarrow e \mu$ or $t \bar{t} \rightarrow e \mu$ receive only modest boosts in the transverse plane. In this case a significant fraction of the events is reconstructed with $x_{\tau_{1,2}}<0$ while many other events end up in the unphysical region $x_{\tau_{1,2}}>1$. The selected events must fulfill the relations

$$
\begin{gathered}
x_{\tau_{1}}>0, x_{\tau_{2}}>0 \\
x_{\tau_{1}}^{2}+x_{\tau_{2}}^{2}<1 .
\end{gathered}
$$

Once the momentum fractions carried by the electron or the muon are estimated the invariant mass of the $\tau \tau$-pair i.e., the invariant mass of the $Z^{0}$ boson is given by

$$
m_{\tau \tau}=m_{e \mu} / \sqrt{x_{\tau_{1}} \cdot x_{\tau_{2}}}
$$

The method has been applied in preliminary $H \rightarrow \tau \tau$ analysis of the ATLAS experiment [99]. 


\section{Appendix B}

\section{Figure of Merit}

The $t \bar{t} \rightarrow e \mu$ cross section is given by,

$$
\sigma=\frac{\mathrm{N}^{\mathrm{Obs}}-\mathrm{N}^{\mathrm{bkg}}}{\mathcal{L} \cdot \epsilon \cdot \mathcal{B R}}
$$

with $\mathrm{N}^{\mathrm{Obs}}$ is the observed number of events, $N^{b k g}$ is the estimated number of background events, $\mathcal{L}$ is the total integrated luminosity of the dataset being analysed, $\epsilon$ is the estimated signal efficiency obtained from $t \bar{t} \rightarrow e \mu$ Monte Carlo, and $\mathcal{B R}$ is the branching ratio for $t \bar{t} \rightarrow e \mu$ decay including the contributions from $W \rightarrow \tau \rightarrow e / \mu$. The error on the cross section 
measurement, can be written as

$$
(\delta \sigma)^{2}=\left(\frac{\partial \sigma}{\partial \mathrm{N}^{\mathrm{Obs}}} \cdot \Delta \mathrm{N}^{\mathrm{Obs}}\right)^{2}+\left(\frac{\partial \sigma}{\partial \mathrm{N}^{\mathrm{bkg}}} \cdot \Delta \mathrm{N}^{\mathrm{bkg}}\right)^{2}+\left(\frac{\partial \sigma}{\partial \epsilon} \cdot \Delta \epsilon\right)^{2}
$$

where the errors of $\mathcal{L}$ and $\mathcal{B R}$ are neglected. Now,

$$
\begin{aligned}
\frac{\partial \sigma}{\partial \mathrm{N}^{\mathrm{Obs}}} & =\frac{1}{\mathcal{L} \cdot \epsilon \cdot \mathcal{B R}} \\
\frac{\partial \sigma}{\partial N^{b k g}} & =\frac{1}{\mathcal{L} \cdot \epsilon \cdot \mathcal{B R}} \\
\frac{\partial \sigma}{\partial \epsilon} & =-\frac{N^{\text {Obs }}-N^{b k g}}{\mathcal{L} \cdot \epsilon^{2} \cdot \mathcal{B R}}
\end{aligned}
$$

Using Eqn. B.3, the Eqn. B.2 can be rewritten as,

$$
\begin{gathered}
(\delta \sigma)^{2}=\left(\frac{\Delta \mathrm{N}^{\mathrm{Obs}}}{\mathcal{L} \cdot \epsilon \cdot \mathcal{B R}}\right)^{2}+\left(\frac{\Delta \mathrm{N}^{\mathrm{bkg}}}{\mathcal{L} \cdot \epsilon \cdot \mathcal{B R}}\right)^{2}+\left(\frac{\mathrm{N}^{\mathrm{Obs}}-\mathrm{N}^{\mathrm{bkg}}}{\mathcal{L} \cdot \epsilon^{2} \cdot \mathcal{B R}} \cdot \Delta \epsilon\right)^{2} \\
\mathrm{~N}^{\mathrm{Obs}}-\mathrm{N}^{\mathrm{bkg}}=S+B-B=S \\
\epsilon \propto S
\end{gathered}
$$


where S and B are the numbers of signal and background events respectively.

Rearranging the terms of Eqn. B.4 it can be rewritten as,

$(\delta \sigma)^{2}=\left(\frac{\mathrm{S}}{\mathcal{L} \cdot \epsilon \cdot \mathcal{B R}} \cdot \frac{\Delta \mathrm{N}^{\mathrm{Obs}}}{\mathrm{S}}\right)^{2}+\left(\frac{\mathrm{S}}{\mathcal{L} \cdot \epsilon \cdot \mathcal{B R}} \cdot \frac{\Delta \mathrm{N}^{\mathrm{bkg}}}{\mathrm{S}}\right)^{2}+\left(\frac{\mathrm{S}}{\mathcal{L} \cdot \epsilon \cdot \mathcal{B R}} \cdot \frac{\Delta \mathrm{S}}{\mathrm{S}}\right)^{2}(\mathrm{~B} .6)$

The observed number of events $\mathrm{N}^{\mathrm{Obs}}$ is assumed to fluctuate according to Poisson hypothesis and thus $\Delta \mathrm{N}^{\text {Obs }}=\sqrt{\mathrm{N}^{\text {Obs }}}=\sqrt{\mathrm{S}+\mathrm{B}}$. The term, $\Delta \mathrm{N}^{\text {bkg }}$ is the total systematic error on background estimation and thus written as $\Delta \mathrm{B}^{\mathrm{syst}}$. The Eqn. B.6 thus becomes,

$$
(\delta \sigma)^{2}=\left(\frac{\mathrm{S}}{\mathcal{L} \cdot \epsilon \cdot \mathcal{B R}}\right)^{2} \cdot\left[\left(\frac{\sqrt{\mathrm{S}+\mathrm{B}}}{\mathrm{S}}\right)^{2}+\left(\frac{\Delta \mathrm{B}^{\mathrm{syst}}}{\mathrm{S}}\right)^{2}+\left(\frac{\Delta \mathrm{S}}{\mathrm{S}}\right)^{2}\right]
$$

Replacing $\frac{\mathrm{S}}{\mathcal{L} \cdot \epsilon \cdot \mathcal{B R}}$ by $\sigma$ from Eqn. B.1 one obtains,

$$
\left(\frac{\delta \sigma}{\sigma}\right)^{2}=\left(\frac{\sqrt{\mathrm{S}+\mathrm{B}}}{\mathrm{S}}\right)^{2}+\left(\frac{\Delta \mathrm{B}^{\mathrm{syst}}}{\mathrm{S}}\right)^{2}+\left(\frac{\Delta \mathrm{S}}{\mathrm{S}}\right)^{2} \text { or, } \frac{\delta \sigma}{\sigma}=\frac{\sqrt{\mathrm{S}+\mathrm{B}}}{\mathrm{S}} \bigoplus \frac{\Delta \mathrm{B}^{\mathrm{syst}}}{\mathrm{S}} \bigoplus \frac{\Delta \mathrm{S}}{\mathrm{S}}
$$




\section{Appendix $\mathrm{C}$}

\section{Candidate Events}

\section{C.1 Event Kinematics}

\begin{tabular}{lccc}
\hline Object & $p_{T}(\mathrm{GeV})$ & $\eta$ & $\phi$ \\
\hline electron & 30.489 & -1.033 & 5.370 \\
$\mu$ & 52.538 & -1.421 & 4.807 \\
jet1 & 58.024 & 0.357 & 0.971 \\
jet2 & 40.383 & -0.159 & 2.204 \\
\hline$E_{T}(\mathrm{GeV})$ & 29.952 & & 3.548 \\
\hline$H_{T}^{\text {leadinglepton }}(\mathrm{GeV})$ & 150.945 & & \\
\hline
\end{tabular}

Table C.1: Kinematics for Event Number:11709332 Run Number:179141 


\begin{tabular}{lccc}
\hline Object & $p_{T}(\mathrm{GeV})$ & $\eta$ & $\phi$ \\
\hline electron & 15.812 & 0.343 & 6.105 \\
$\mu$ & 51.984 & 1.213 & 4.242 \\
jet1 & 114.031 & -0.049 & 5.016 \\
jet2 & 48.479 & -0.19 & 0.444 \\
\hline$E_{T}(\mathrm{GeV})$ & 151.184 & & 1.994 \\
\hline$H_{T}^{\text {leadinglepton }}(\mathrm{GeV})$ & 214.496 & & \\
\hline
\end{tabular}

Table C.2: Kinematics for Event Number:8735139 Run Number:178733

\begin{tabular}{lccc}
\hline Object & $p_{T}(\mathrm{GeV})$ & $\eta$ & $\phi$ \\
\hline electron & 73.163 & -0.659 & 6.194 \\
$\mu$ & 76.789 & -0.252 & 3.562 \\
jet1 & 110.555 & -0.152 & 0.421 \\
jet2 & 109.007 & -0.432 & 2.501 \\
\hline$E_{T}(\mathrm{GeV})$ & 70.269 & & 4.417 \\
\hline$H_{T}^{\text {leadinglepton }}(\mathrm{GeV})$ & 296.35 & & \\
\hline
\end{tabular}

Table C.3: Kinematics for Event Number:26386170 Run Number:179195

\begin{tabular}{lccc}
\hline Object & $p_{T}(\mathrm{GeV})$ & $\eta$ & $\phi$ \\
\hline electron & 39.064 & -0.175 & 3.489 \\
$\mu$ & 39.321 & 0.747 & 3.271 \\
jet1 & 129.166 & -0.108 & 0.156 \\
jet2 & 80.068 & 1.384 & 3.027 \\
\hline$E_{T}(\mathrm{GeV})$ & 29.127 & & 5.923 \\
\hline$H_{T}^{\text {leadinglepton }}(\mathrm{GeV})$ & 248.554 & & \\
\hline
\end{tabular}

Table C.4: Kinematics for Event Number: 19617819 Run Number: 179331 


\begin{tabular}{lccc}
\hline Object & $p_{T}(\mathrm{GeV})$ & $\eta$ & $\phi$ \\
\hline electron & 51.324 & -1.091 & 2.086 \\
$\mu$ & 80.209 & -0.493 & 5.589 \\
jet1 & 163.324 & -0.098 & 5.05 \\
jet2 & 117.705 & -0.361 & 2.376 \\
\hline$\not_{T}(\mathrm{GeV})$ & 77.918 & & 1.615 \\
\hline$H_{T}^{\text {leadinglepton }}(\mathrm{GeV})$ & 361.237 & & \\
\hline
\end{tabular}

Table C.5: Kinematics for Event Number: 15259654 Run Number: 177826

\begin{tabular}{lccc}
\hline Object & $p_{T}(\mathrm{GeV})$ & $\eta$ & $\phi$ \\
\hline electron & 109.278 & -0.641 & 4.055 \\
$\mu$ & 123.383 & 0.174 & 0.963 \\
jet1 & 65.791 & 0.367 & 0.367 \\
jet2 & 44.829 & 0.341 & 3.186 \\
\hline$\not_{T}(\mathrm{GeV})$ & 40.696 & & 4.321 \\
\hline$H_{T}^{\text {leadinglepton }}(\mathrm{GeV})$ & 234.003 & & \\
\hline
\end{tabular}

Table C.6: Kinematics for Event Number: 37315438 Run Number:178159

\begin{tabular}{lccc}
\hline Object & $p_{T}(\mathrm{GeV})$ & $\eta$ & $\phi$ \\
\hline electron & 15.944 & 1.067 & 2.292 \\
$\mu$ & 56.599 & -0.368 & 1.421 \\
jet1 & 78.639 & 0.381 & 4.862 \\
jet2 & 50.691 & -0.839 & 2.723 \\
\hline$\not_{T}(\mathrm{GeV})$ & 38.426 & & 6.082 \\
\hline$H_{T}^{\text {leadinglepton }}(\mathrm{GeV})$ & 185.93 & & \\
\hline
\end{tabular}

Table C.7: Kinematics for Event Number: 1997007 Run Number: 168393 


\begin{tabular}{lccc}
\hline Object & $p_{T}(\mathrm{GeV})$ & $\eta$ & $\phi$ \\
\hline electron & 136.531 & -0.042 & 1.898 \\
$\mu$ & 29.588 & 0.493 & 1.659 \\
jet1 & 92.324 & 0.795 & 5.171 \\
jet2 & 91.419 & 1.514 & 4.676 \\
jet3 & 47.079 & 0.721 & 3.719 \\
jet4 & 34.712 & -2.993 & 2.293 \\
\hline ET $_{T}(\mathrm{GeV})$ & 84.358 & & 0.053 \\
\hline$H_{T}^{\text {leadinglepton }}(\mathrm{GeV})$ & 402.066 & & \\
\hline
\end{tabular}

Table C.8: Kinematics for Event Number: 8710859 Run Number: 174901 


\section{C.2 Event Display}



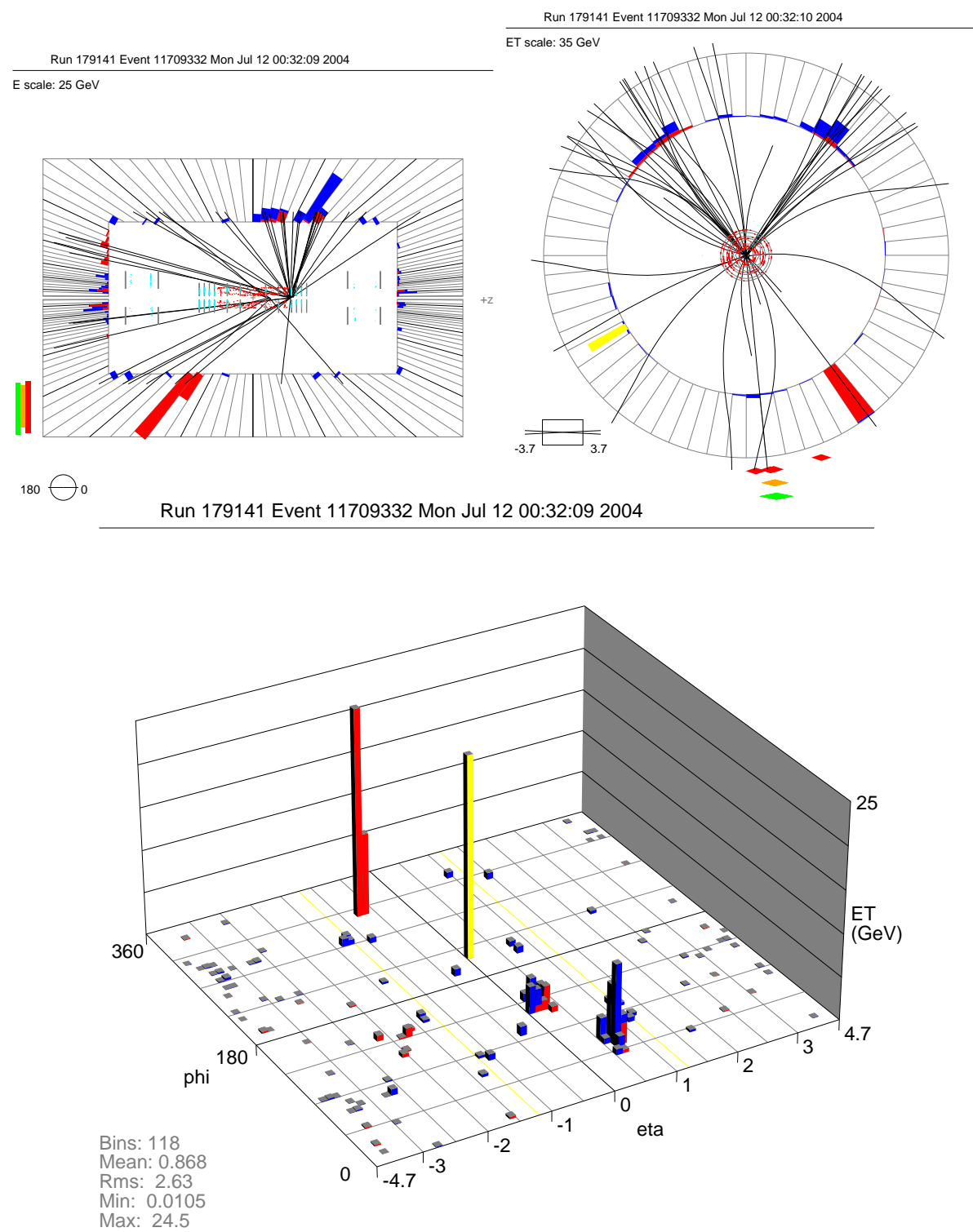

$\mathrm{mE} \mathrm{t}: 23.7$

phi_t: 211 deg

Figure C.1: $e-\mu$ candidate event: Run 179141 Event 11709332; RZ view (upper right), XY view (upper left), Lego view (lower). 


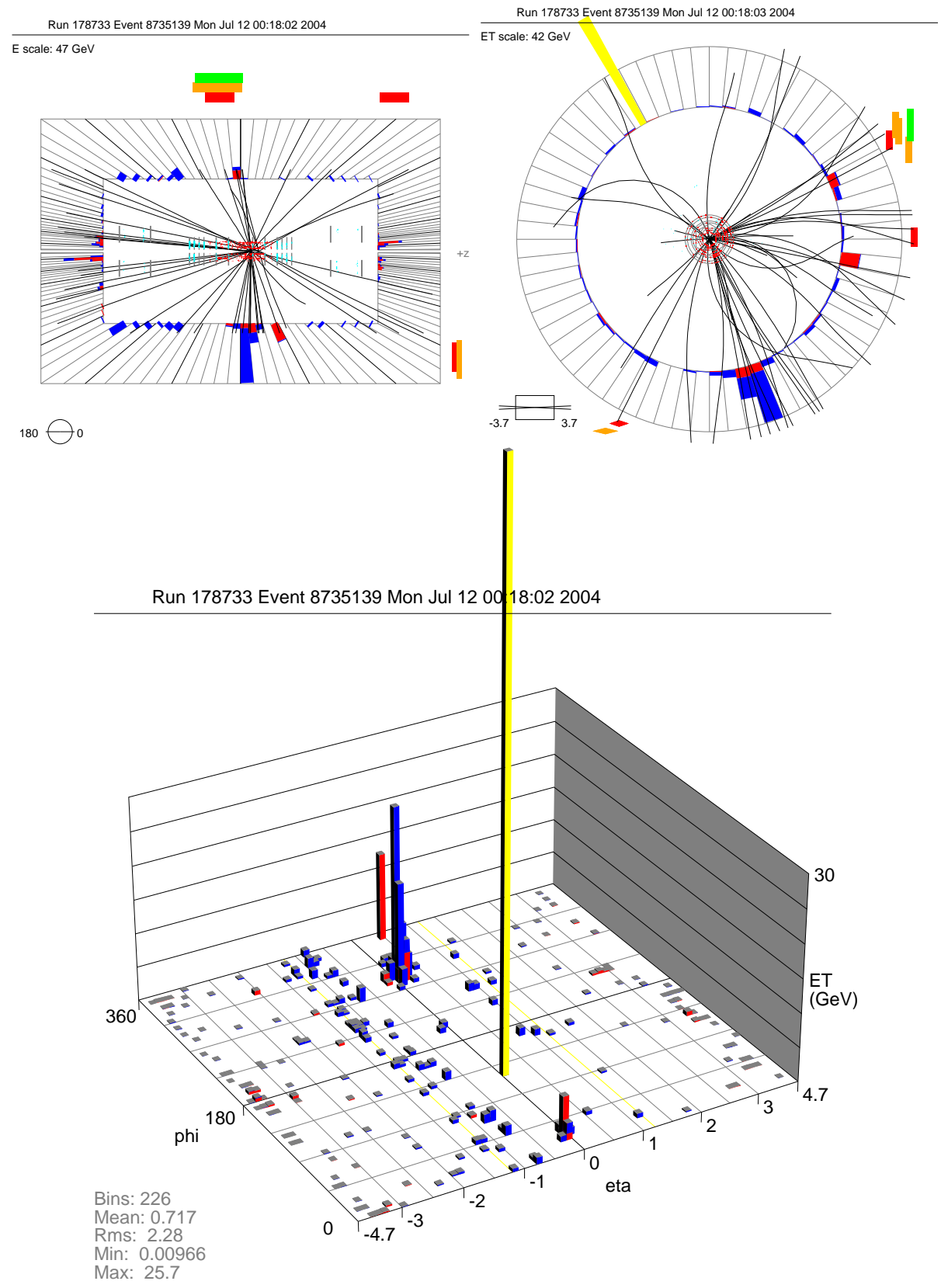

mE_t: 85.1
phi_t: 120 deg

Figure C.2: $e-\mu$ candidate event: Run 178733 Event 8735139; RZ view (upper right), XY view (upper left), Lego view (lower). 

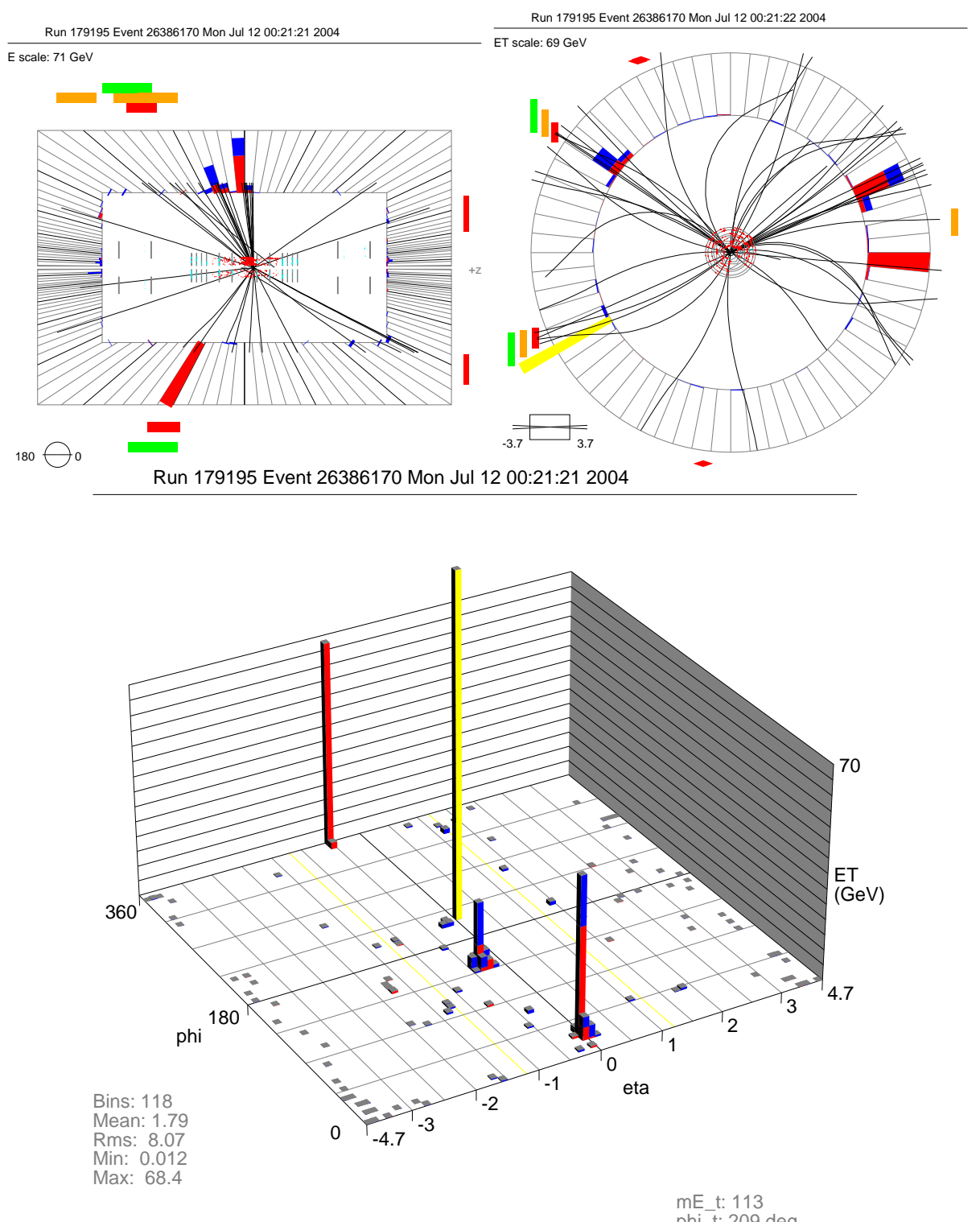

Figure C.3: $e-\mu$ candidate event: Run 179195 Event 26386170; RZ view (upper right), XY view (upper left), Lego view (lower). 

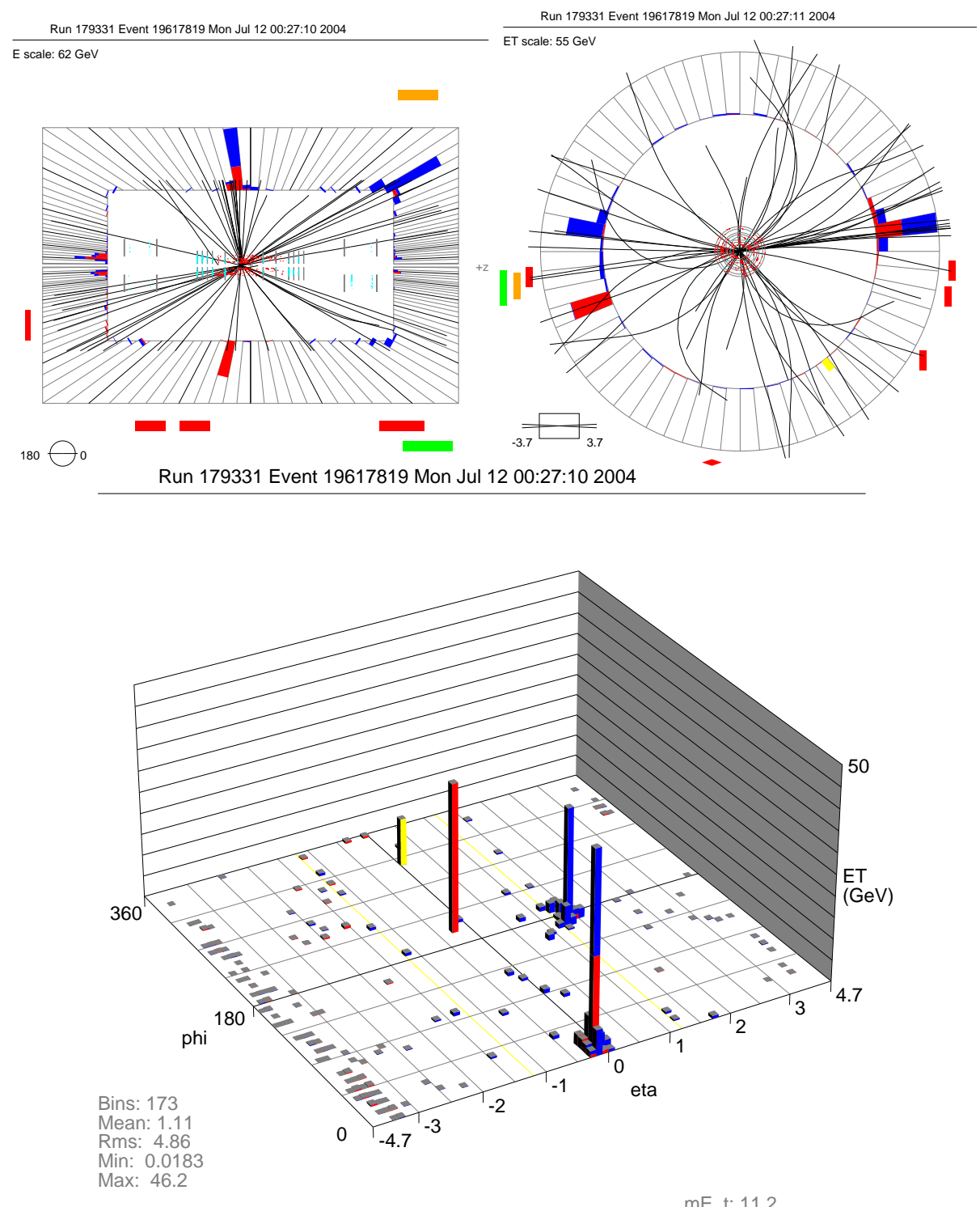

phi t: $308 \mathrm{deg}$

Figure C.4: $e-\mu$ candidate event: Run 179331 Event 19617819; RZ view (upper right), XY view (upper left), Lego view (lower). 

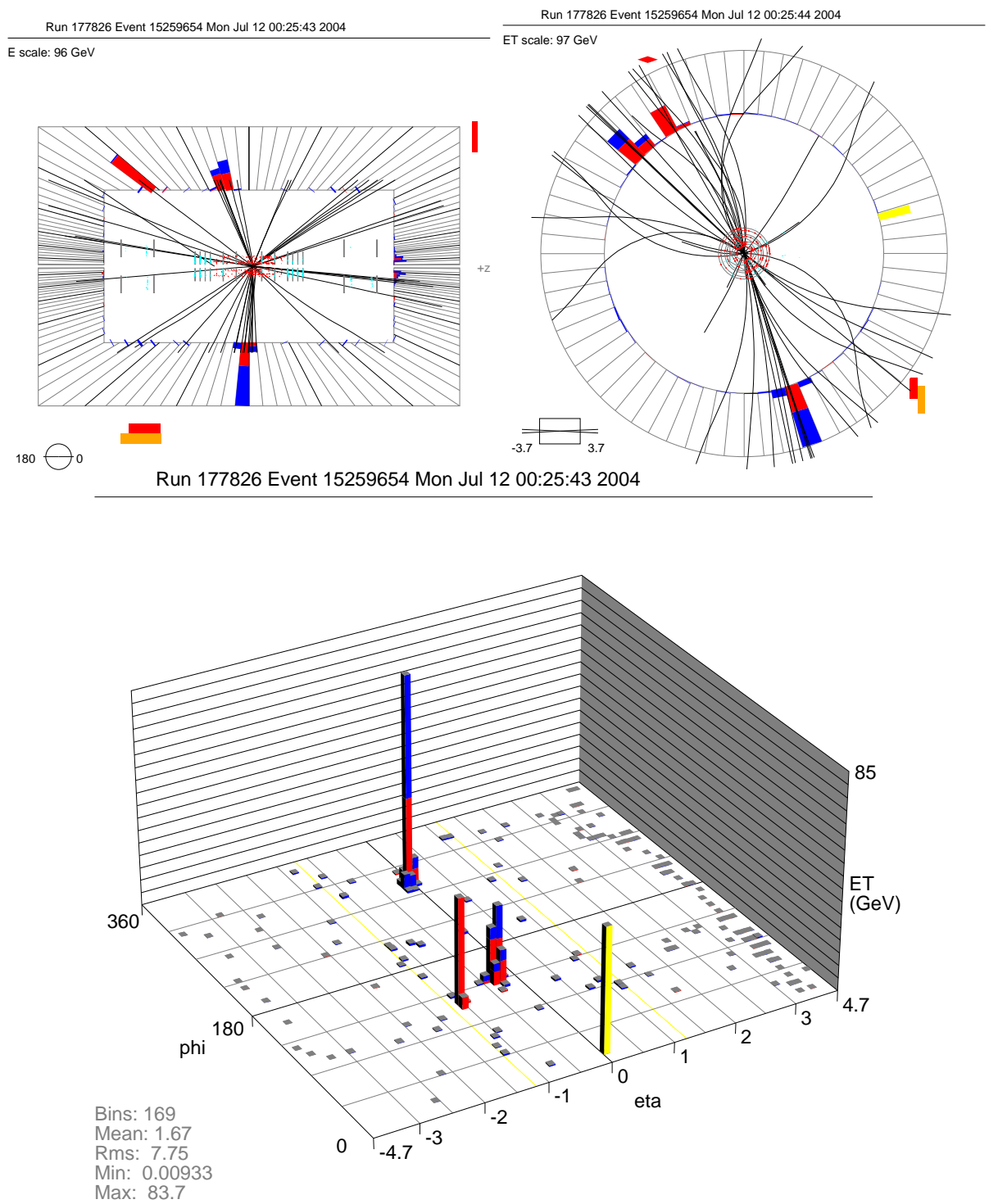

$\mathrm{mE} t: 49.3$

Figure C.5: $e-\mu$ candidate event: Run 177826 Event 15259654; RZ view (upper right), XY view (upper left), Lego view (lower). 

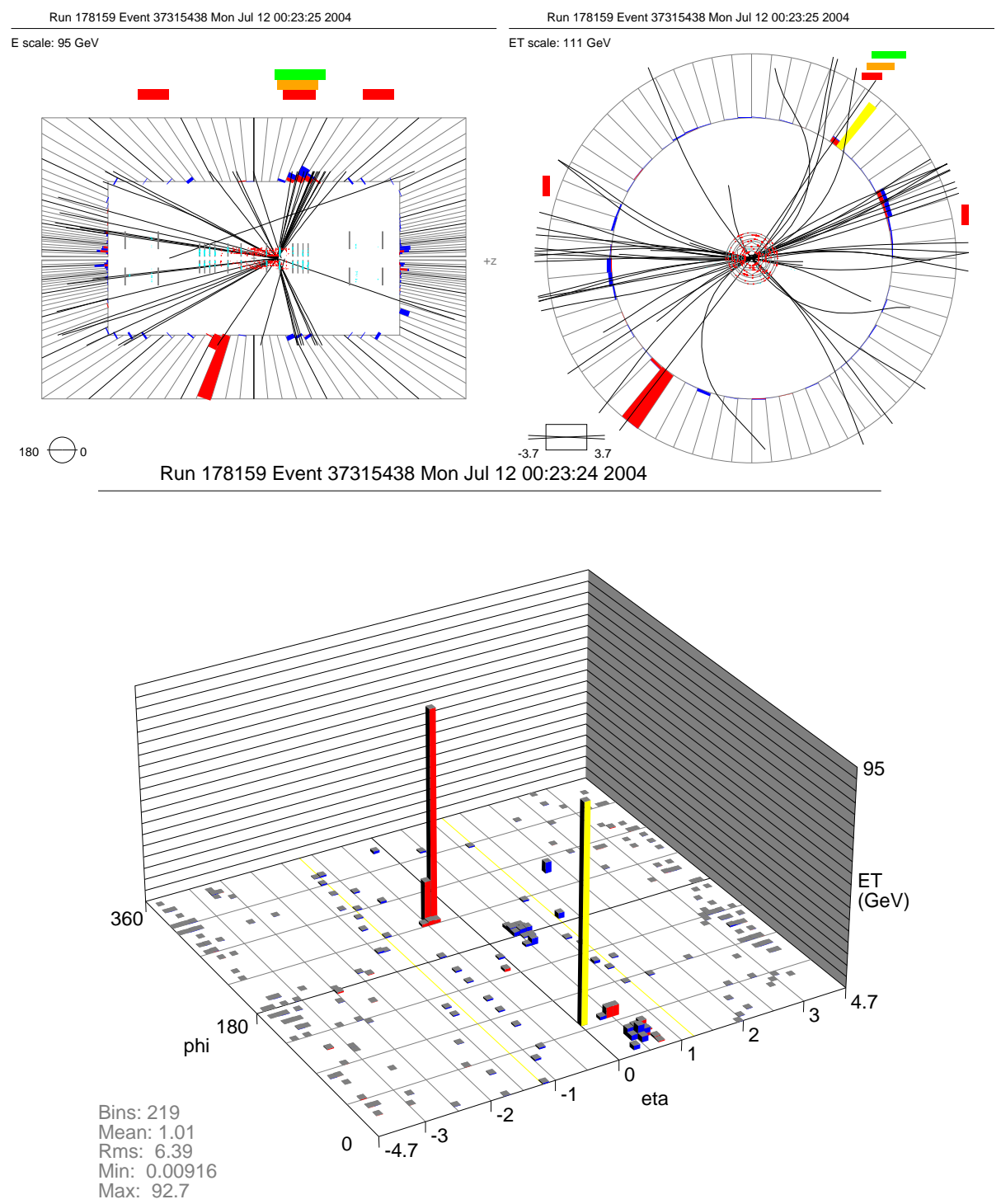

mE_t: 95.5

Figure C.6: $e-\mu$ candidate event: Run 178159 Event 37315438; RZ view (upper right), XY view (upper left), Lego view (lower). 

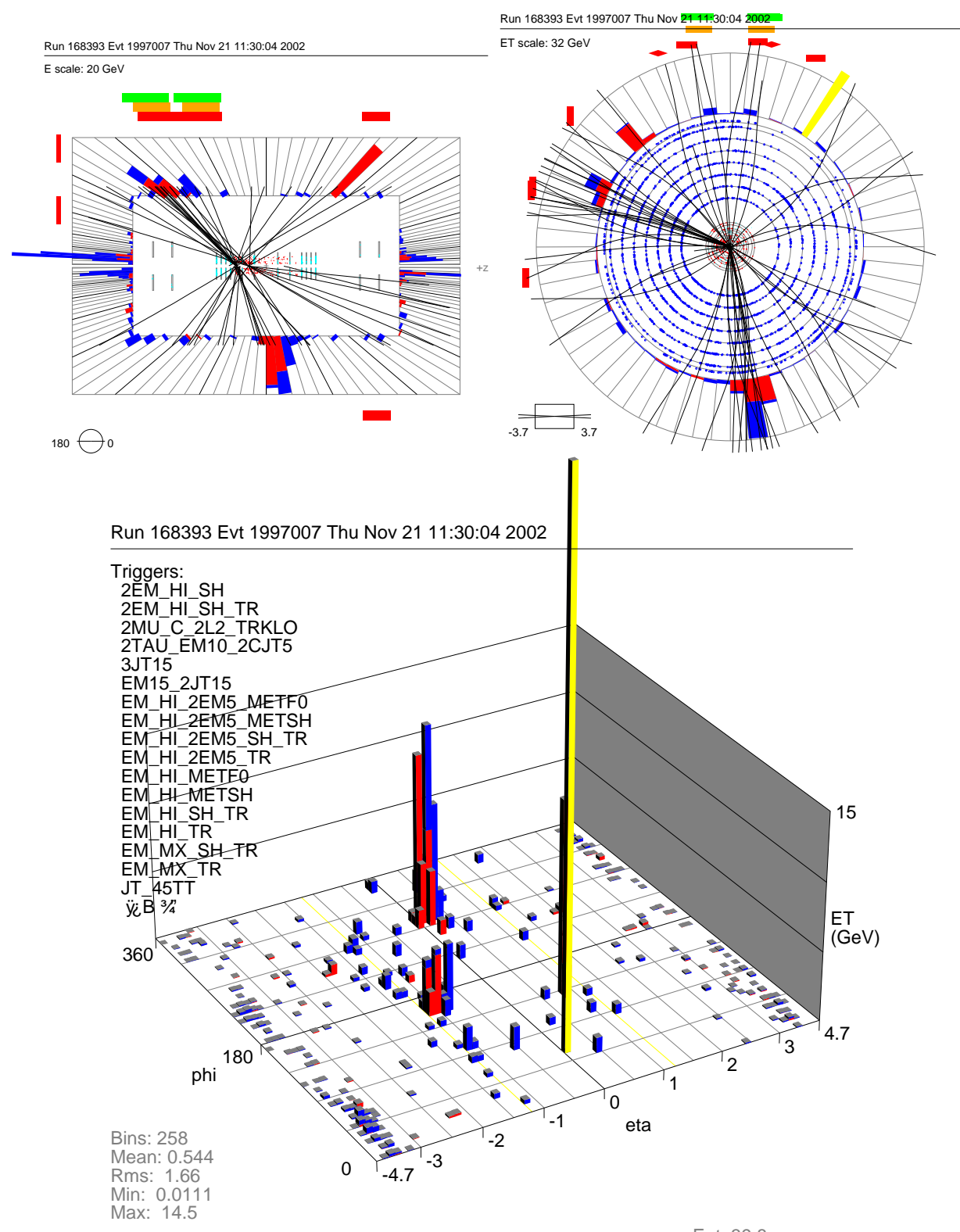

mE_t: 39.8
phi_t: $56.3 \mathrm{deg}$

Figure C.7: $e-\mu$ candidate event: Run 168393 Event 1997007 ; RZ view (upper right), XY view (upper left), Lego view (lower). 

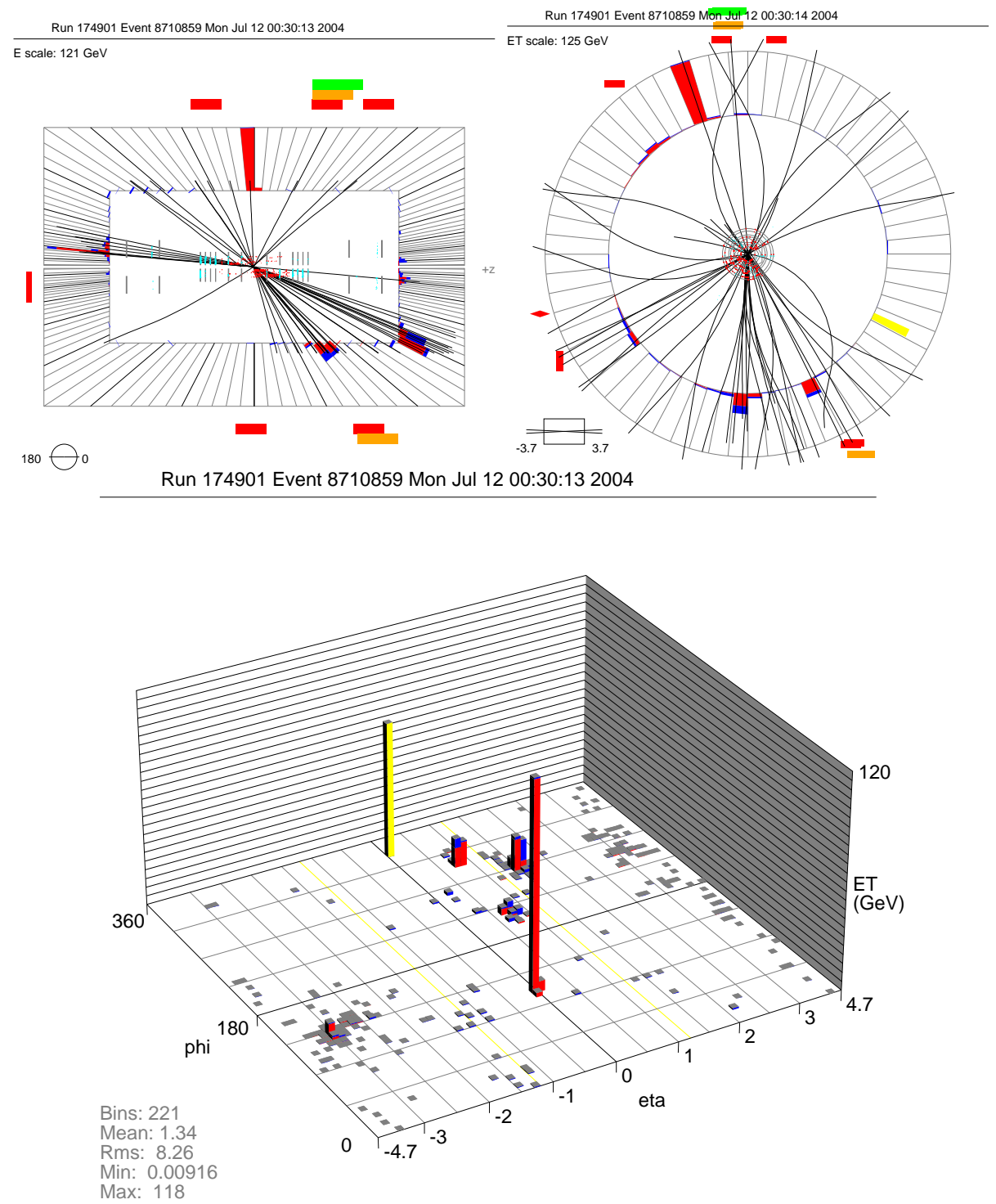

mE_t: 76.7

phi t: 334 deg

Figure C.8: $e-\mu$ candidate event: Run 174901 Event 8710859 ; RZ view (upper right), XY view (upper left), Lego view (lower). 


\section{Bibliography}

[1] J. Thomson, Philosophical Magazine, 44, series 5, 293 (1897).

[2] E. Rutherford, Proc. Roy. Soc., A82, 492 (1910).

[3] N. Bohr, Philosophical Magazine, 26, 1 (1913).

[4] J. Chadwick, Nature 129, 312 (1932).

[5] P. A. M. Dirac, Proc. Roy. Soc., A114, 710 (1927).

[6] R. P. Feynman, Phys. Rev., 76, 749 (1949).

[7] E. Fermi, Z. Physics, 88, 161 (1934).

[8] C.L. Cowan, Jr., F. Reines, F.B. Harrison, H.W. Kruse and A.D. McGuire, Science, 124, 103 (1956);

Frederick Reines and Clyde L. Cowan, Jr., Nature, 178, 446 (1956)

Frederick Reines and Clyde L. Cowan, Jr., Physics Today, 10, 8, 12 (1957).

[9] Y. Yukawa, Proc. Phys.- Math. Soc., Japan, 17, 48 (1935).

[10] C. M. G. Lattes et al., Nature, 159, 694 (1947); 160, 453, 486 (1947).

[11] Murry Gell-Mann, Phys. Lett., 8, 214 (1964).

[12] DONUT Collaboration, Phys. Lett. B, 504, 218 (2001).

[13] S. Glashow, Nuclear Physics, 22, 579 (1961);

A. Salam and J. C. Ward, Phys. Lett., 13, 168 (1964).

S. Weinberg, Phys. Lett., 19, 1264 (1967). 
[14] UA1 Collaboration, Phys. Lett. B, 122, 103 (1983);

UA1 Collaboration, Phys. Lett. B, 122, 476 (1983);

UA2 Collaboration, Phys. Lett. B, 126, 398 (1983);

UA2 Collaboration, Phys. Lett. B, 129, 130 (1983).

[15] Barger V. D., Phillips R. J., "Collider Physics", Addison-Wesley (1987); F. Halzen and A. D. Martin, "Quarks and Leptons", John Wiley \& Sons (1984); David Griffiths, "Introduction to Elementary Particles", John Wiley \& Sons, Inc. (1987).

[16] Jonathan L. Rosner, American J. Phys., 71, 302 (2003); hep-ph/0206006.

[17] M. L. Perl et al., Phys. Rev. Lett., 35, 1489 (1975).

[18] S. W. Herb et al., Phys. Rev. Lett., 39, 252 (1977).

[19] S. Abachi et al. (DØ Collaboration), Phys. Rev. Lett., 74, 2632 (1995).

[20] F. Abe et al. (CDF Collaboration), Phys. Rev. Lett., 74, 2626 (1995).

[21] The CDF Collaboration, the D $\varnothing$ Collaboration and the TEVATRON ElectroWeak Working Group, Combination of CDF and DØ Results on Top-Quark Mass; hep-ex/0404010.

[22] T. Affolder et al. (CDF Collaboration), Phys. Rev. Lett., 86, 3233 (2001); hep-ex/0012029.

[23] DØ Collaboration, "Simultaneous measurement of $\mathcal{R}=\mathcal{B}(\mathrm{t} \rightarrow \mathrm{Wb}) / \mathcal{B}(\mathrm{t} \rightarrow-$ $\mathrm{Wq})$ and $\sigma(p \bar{p} \rightarrow t \bar{t})$ at $\mathrm{D} \varnothing ", \mathrm{D} \varnothing$ note $4833-\mathrm{CONF}$;

URL: http://www-d0.fnal.gov/Run2Physics/top/top_public_web_pages/ conference_notes/summer05_prop_ljets_brsigma_confnote.pdf

[24] B. Abbott et al. (DØ Collaboration), Phys. Rev. Lett., 85, 256 (2000); FERMILAB-Pub-00/046-E, hep-ex/0002058; Suyong Choi, Spin Correlation in $t \bar{t}$ Production from $p \bar{p}$ Collisions at $\sqrt{s}=1.8 \mathrm{TeV}$, Ph.D. thesis submitted at Seoul National University, Korea (1999).

[25] J. Collins et al., Nucl. Phys. B, 263, 37 (1986).

[26] R. Bonciani, S. Catani, M. L. Mangano and P. Nason, Nucl. Phys. B, 529, 424 (1998); hep-ph/9801375.

[27] M. Glück, J. F. Owens and E. Reya, Phys. Rev. D, 17, 2324 (1978);

B. L. Combridge, Nucl. Phys. B, 151, 429 (1979);

J. Babcock, D. Sivers and S. Wolfram, Phys. Rev. D, 18, 162 (1978); 
K. Hagiwara and T. Yoshino, Phys. Lett. B, 80, 282 (1979);

L. M. Jones and H. Wyld, Phys. Rev. D, 17, 782 (1978);

H. Georgi et al., American J. Phys., 114, 273 (1978).

[28] G. Sterman, Nucl. Phys. B, 281, 310 (1987);

S. Catani and L. Trentadue, Nucl. Phys. B, 327, 323 (1989);

N. Kidonakis, Int. Journal of Mod. Phys., A19, 1793 (2004); hep-ph/0303186;

N. Kidonakis, Mod. Phys. Lett. A, 19, 405 (2004); hep-ph/0401147.

[29] M. Cacciari et al., J. High Energy Phys., 404, 68 (2004); hep-ph/0303085.

[30] N. Kidonakis et al., Phys. Rev. D, 68, 114014 (2003); hep-ph/0308222.

[31] G. Eilam et al., Phys. Rev. Lett., 66, 3105 (1991).

[32] S. Eidelman et al., Phys. Lett. B, 592, 1 (2004).

[33] D. Chakraborty et al., Ann. Rev. Nucl. Part. Sci., 53, 301 (2003); hep$\mathrm{ph} / 0303092$.

[34] J.D. Cockroft, E.T.S. Walton, Proc. of the Royal Soc. of London, A136, 619 (1932).

[35] "Run II handbook"; URL: http://www-ad.fnal.gov/runII/index.html.

[36] S. Abachi et al., "The DØ upgrade: The Detector and its physics", FERMILAB-PUB-96-357-E.

[37] T. Edwards et al., "Determination of the Effective Inelastic $p \bar{p}$ Cross-section for the DØ Run II Luminosity Measurement", FERMILAB-TM-2278-E.

[38] M. Roco, "The Silicon Microstrip Tracker for the DØ Upgrade", D $\varnothing$ note 3553 (1998).

[39] E. Kajfasz (for DØ collaboration), Nucl. Instrum. Meth. A, 511, 16 (2003); hep-ex/0112014.

[40] "The DØ Upgrade Central Fibre Tracker, Technical Design Report (1997)"; URL: http://d0server1.fnal.gov/projects/SciFi/cft home.html.

[41] Alexander Khanov, Measurement of the $t \bar{t}$ Production Cross-Section as $\sqrt{s}=$ $1.96 \mathrm{TeV}$ Using Lifetime Tagging, Ph.D. thesis submitted at University of Rochester (2004).

[42] "Design Report of Central Preshower Detector for the DØ Upgrade" (1996). 
[43] A. Gordeev et al., "Technical Design Report of the Forward Preshower Detector for the DØ Upgrade, DØ note 3445 (1998).

[44] "Calorimeter Electronics Upgrade for Run 2, Technical Design Report (1998)";

URL: http://www-d0.fnal.gov/hardware/cal/intro/tdr/tdrformat.htm .

[45] DØ Collaboration, S. Abachi et al., Nucl. Inst. Meth. A, 338, 185 (1994).

[46] DØ Collaboration, S. Abachi et al., Nucl. Inst. Meth. A, 324, 53 (1993).

[47] Scott S. Snyder, Measurement of the top quark mass at DØ, Ph.D. thesis submitted at State University of New York at Stony Brook, New York (1995).

[48] J. Butler et al., "The DØ Muon System Upgrade" DØ note 2780 (1996).

[49] B. Baldin et al., "Technical Design of the Central Muon System", DØ note 3365 (1997).

[50] G. Alexeev et al., "Technical Design Report for the DØ Forward Muon Tracking Detector Based on Mini-drift Tubes", DØ note 3366 (1997).

[51] Andrew Askew, "Parametrizing the Local Muon Resolution in p13 Data for PMCS", DØ note 4314 (2003).

[52] M. Abolins et al., "DØ Run II Level 1 Trigger Framework Technical Design Report", DØ note 328 (June, 1998); URL: http://www.pa.msu.edu/hep/d0/ftp/11/framework/11fw_tdr_05june98.txt.

[53] "DØ Run II Level 2 Trigger";

URL: http://www.pa.msu.edu/hep/d0/12/index.html .

[54] D. Edmunds et al., "Technical Design Report for the Level 2 Global Processor", DØ note 3402 (1998).

[55] A. Boehnlein et al., "Description of the DØ L3 Trigger software components", DØ note 3630 (1999).

[56] C. Schmitt, "Split CFT Clusters", DØ note 4092 (2003).

[57] P.V.C. Hough, "Machine Analysis of Bubble Chamber Pictures", Int. Conf. on High Energy Accelerators and Instrumentation, CERN (1959).

[58] R. Fruhwirth, Nucl. Ins. Meth. A, 262, 444 (1987). 
[59] A. Khanov, "HTF: histogramming method for finding tracks; the algorithm description", DØ note 3778 (2000).

[60] G. Borissov, "Technical Details of AA Tracking", presented at All DØ meeting, February 28, 2003.

[61] G. Watts et al., "Primary Vertex Certification in p14", DØ note 4320 (2004).

[62] A. Schwartzman, "Vertex fitting and finding in ROOT"; URL: http://www-d-

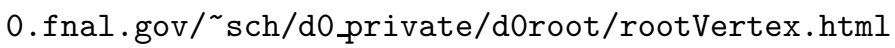

[63] M. Narain et al., "Primary Vertex Selection", DØ note 3906 (2001).

[64] S. Abachi et al., Phys. Rev. D, 52, 4877 (1995).

[65] J. Kozminski et al., "Electron Likelihood in p14", DØ note 4449 (2004).

[66] Elemer Nagy et al., "Simultaneous Calibration of Various Parts of the DØ Electromagnetic Calorimeter", DØ note 3758 (2000).

[67] "DØ Run II electron/photon ID certification"; URL: http://www-do.fnal.gov/phys_id/emid/d0_private/certification/main_v5_0.html.

[68] D. Whiteson et al., "Muon Isolation Studies", DØ note 4070 (2003).

[69] J. E. Huth et al., Proceedings of Research Directions for the Decade: Snowmass 1990, 134 (July, 1990).

[70] G. C. Blazey et al., "Run II Jet Physics: Proceedings of the Run II QCD and Weak Boson Physics Workshop (2000)"; hep-ex/0005012.

[71] "Jet Energy Scale and Resolutions"; URL: http://www-d0.fnal.gov/phys_id/jes/d0_private/jes.html.

[72] M. Begel et al., "Luminosity and Performance of DØ during the First Year of Run 2", DØ note 3973 (2002).

[73] "Run Quality database";

URL: http://d0db.fnal.gov/qualitygrabber/qualQueries.html.

[74] P. K. Mal et al., "DØ Luminosity in Run 2: Reconstructed", DØ note 4438 (2004).

[75] M. Agelou et al., "Top Trigger Efficiency Measurements and the top_trigger package", D $\varnothing$ note 4512 (2004). 
[76] M.L. Mangano, M. Moretti, F. Piccinini, R. Pittau, A. Polosa, JHEP, 0307, 1 (2003); hep-ph/0206293; M.L. Mangano, M. Moretti, R. Pittau, Nucl. Phys. B, 632, 343 (2002); hep-ph/0108069; F. Caravaglios, M. L. Mangano, M. Moretti, R. Pittau, Nucl. Phys. B, 539, 215 (1999), hep-ph/9807570.

[77] T. Sjöstrand, P. Edén, C. Friberg, L. Lönnblad, G. Miu, S. Mrenna and E. Norrbin, Computer Physics Commun., 135, 238 (2001).

[78] D. Lange, A. Ryd et al., The EvtGen Event Generator Package, Proceedings of CHEP (1998).

[79] S. Jadach, Z. Was, R. Decker, J.H. Kuehn, The tau decay library TAUOLA: version 2.4 QED corrections in decay modes, Comp. Phys. Commun., 76, 361 (1993); CERN TH-6793.

[80] DØ Collaboration, "DØ Measurement of $\sigma_{\mathrm{Z}^{0}} \times \mathrm{BR}\left(\mathrm{Z}^{0} \rightarrow \mu \mu\right)$ ", Sep. 12, 2003; URL: http://www-d0.fnal.gov/Run2Physics/wz/Public/results/r2zmumucs_summer03.html .

[81] J.M. Campbell and R.K. Ellis, Phys Rev D, 60, 113006 (1999).

[82] M. Agelou et al., "Measurement of the Inclusive $W \rightarrow \mu \nu$ Cross-Section in $p \bar{p}$ Collisions at $\sqrt{s}=1.96 \mathrm{TeV} "$, DØ note 4128 (2003).

[83] U. Baur, S. Keller, D. Wackeroth, FERMILAB-CONF-96/424-T, hepph/9611332; U. Baur, D. Wackeroth, Radcor 2000 proceedings contribution, hep-ph/0101254; U. Baur, D. Wackeroth, DPF 2000 Proceedings contribution, hep-ph/0011080.

[84] R. Brun and F. Carminati, CERN program library long writeup, W5013 (1993).

[85] Prolay Mal et al., "Luminosity Measurement with D $\varnothing$ Tracker", DØ note 4547 (2004).

[86] S. Jain, "Scale and Over-smearing for high $p_{T}$ MC electrons", DØ note 4402 (2004).

[87] Top Physics Group of DØ Collaboration, "DØ Top Analysis and Data Sample for the Winter Conferences 2004", DØ note 4419 (2004).

[88] P. Mal et al., "Optimization of $t \bar{t} \rightarrow e \mu j j$ selection criteria", DØ note 4437 (2004).

[89] S. Anderson et al., "Measurement of the $t \bar{t}$ production cross section in dilepton final states at $\sqrt{s}=1.96 \mathrm{TeV} "$, D $\varnothing$ note 4420; URL: http://wwW-d0.fnal.gov/Run2Physics/WWW/results/prelim/TOP/T03/T03.pdf. 
[90] Tobias Golling, "Measurement of the $t \bar{t}$ production Cross section at at $\sqrt{s}=1.96$, in the Muon+Jets Final State using a topological method"; URL: http://www-d0.fnal.gov/Run2Physics/top/private/Notes/mujets_topo_note_v1_0.pdf.

[91] E.W. Varnes, "Track reconstruction efficiency measurement with single muons", DØ Note 4317 (2003).

[92] S. Banerjee et al., "Discrepancy in the existing Monte Carlo description of the Silicon Tracker in DØ detector", DØ note 4560 (2004).

[93] V. M. Abazov et al. (DØ Collaboration), Phys. Lett. B, 626, 55 (2005); hep-ex/0505082.

[94] DØ Collaboration, V. M. Abazovet al., Phys. Rev. D, 67, 012004 (2003).

[95] V. M. Abazov et al. (DØ Collaboration), Phys. Lett. B, 626, 45 (2005); hep-ex/0504043.

[96] V. M. Abazov et al. (DØ Collaboration), Phys. Lett. B, 626, 35 (2005); hep-ex/0504058.

[97] D $\varnothing$ Collaboration, "Combined $t \bar{t}$ Production Cross Section at $\sqrt{s}=1.96 \mathrm{TeV}$ in the Lepton+jets and Dilepton Final States using Event Topology"

URL: http://www-d0.fnal.gov/Run2Physics/top/top_public_web_pages/conference_notes/

summer05_xsec_lj-11_combined_confnote.pdf

[98] D. Acosta et al. (CDF Collaboration), Phys. Rev. Lett., 93, 142001 (2004).

[99] S. Asai et al., Prospects for the Search for a Standard Model Higgs Boson in ATLAS using Vector Boson Fusion, hep-ph/0402254. 